\title{
Structural Health Monitoring of Adhesively Bonded Composite Joints
}

\author{
By \\ Fady Habib B.Eng \\ A Thesis Submitted to \\ The Faculty of Graduate Studies and Research \\ In partial fulfilment of \\ the degree requirements of \\ Master of Applied Science \\ Ottawa-Carleton Institute for \\ Mechanical and Aerospace Engineering \\ Department of Mechanical and Aerospace Engineering \\ Carleton University \\ Ottawa, Ontario, Canada \\ January 2012
}

Copyright (c)

2012- Fady Habib 
Library and Archives

Canada

Published Heritage

Branch

395 Wellington Street

Ottawa ON K1A ON4

Canada
Bibliothèque et

Archives Canada

Direction du

Patrimoine de l'édition

395 , rue Wellington

Ottawa ON K1A ON4

Canada
Your file Votre référence

ISBN: 978-0-494-91573-8

Our file Notre référence

ISBN: $978-0-494-91573-8$
NOTICE:

The author has granted a nonexclusive license allowing Library and Archives Canada to reproduce, publish, archive, preserve, conserve, communicate to the public by telecommunication or on the Internet, loan, distrbute and sell theses worldwide, for commercial or noncommercial purposes, in microform, paper, electronic and/or any other formats.

The author retains copyright ownership and moral rights in this thesis. Neither the thesis nor substantial extracts from it may be printed or otherwise reproduced without the author's permission.
AVIS:

L'auteur a accordé une licence non exclusive permettant à la Bibliothèque et Archives Canada de reproduire, publier, archiver, sauvegarder, conserver, transmettre au public par télécommunication ou par l'Internet, prêter, distribuer et vendre des thèses partout dans le monde, à des fins commerciales ou autres, sur support microforme, papier, électronique et/ou autres formats.

L'auteur conserve la propriété du droit d'auteur et des droits moraux qui protege cette thèse. $\mathrm{Ni}$ la thèse ni des extraits substantiels de celle-ci ne doivent être imprimés ou autrement reproduits sans son autorisation.
In compliance with the Canadian Privacy Act some supporting forms may have been removed from this thesis.

While these forms may be included in the document page count, their removal does not represent any loss of content from the thesis.
Conformément à la loi canadienne sur la protection de la vie privée, quelques formulaires secondaires ont été enlevés de cette thèse.

Bien que ces formulaires aient inclus dans la pagination, il n'y aura aucun contenu manquant. 


\begin{abstract}
In recent years, many aerospace organizations have researched and implemented composite materials to achieve better fuel efficiency as well as reduced maintenance cost. In addition to the use of composites, manufacturers are investigating the use of adhesive bonded joints and composite patch bonded repairs to extend the life of their in-service aircraft. Adhesive joints are superior to traditional mechanical fasteners as they reduce stress concentration zones and overall part count. However, the integrity of an adhesive joint is difficult to inspect. Inspection of adhesive joints may be carried out using interrogation technology such as Structural Health Monitoring (SHM). This thesis focuses on the evaluation of Acoustic-Ultrasonic (AU) SHM technique for the detection of crack and disbond growth. In addition to AU, Capacitance Disbond Detection Technique (CDDT) and the Surface Mountable Crack Detection System (SMCDS) were evaluated for the detection disbonds. Results of the AU system demonstrated that AU technology may be used to detect and quantify crack and disbond growth. It was also found that SMCDS and CDDT both complement each other, as SMCDS identified the location of disbond while CDDT quantify disbond.
\end{abstract}




\section{Acknowledgments}

During the course of my master's degree I have had the pleasure of working with many knowledgeable individuals from both the National Research Council Canada (NRC) and Carleton University. I would like to thank all of them for their guidance, support and their patience. In the event that I forget to mention your name below, I truly apologize. Before I begin to thank all the individuals I have worked with over these years, I would like to start by thanking those who have always been there for me since my birth; My Family.

Of all the individuals I have worked with, I would like to thank both my supervisors: Prof. Martinez and Prof. Artemev for providing me with guidance and knowledge. I would mostly like to thank them for spending a great deal of time reviewing my 200 page thesis; I know it wasn't easy. Prof. Martinez was unlike any professor that I have ever had. He was not just my supervisor for these past two years but was also a good friend.

I would like to extend my thanks to: Tom Benak, Tom Kay, Brian Moyes, Mike Brothers, Marc Genest, and Michel Delannoy for their aid in manufacturing and testing of all my coupons. I would also like to thank Dr. Guillaume Renaud for his AFGROW analysis that he conducted for me.

I would also like to thank two of my closest friends Mario and Shashank for their friendship over this past little while. The part I regret the most during my academic career was, not knowing you guys during my undergrad years. We have spent many hours discussing academia, research and life objects over coffee, food and sometimes beer. I think that if we add up all our individual hours spent together, it would probably add up to how much time a person takes to finish a Ph.D. Also, thank you Shashank for spending time with me to explain Lamb Wave theory. 
For financial support, I would like to thank NRC, Carleton University and MITACS. All of their funding has played an important role in the testing of advanced SHM technologies. More importantly, it has helped me expand my knowledge and personal growth. 


\section{Table of Contents}

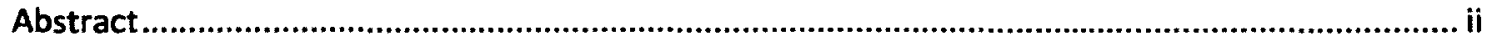

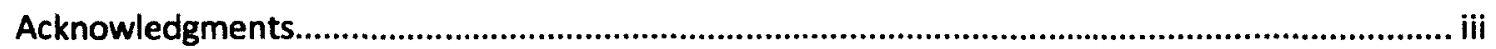

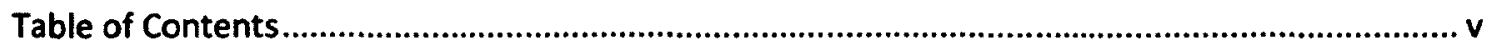

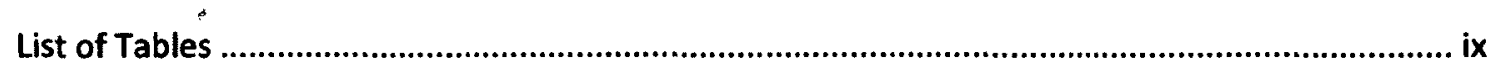

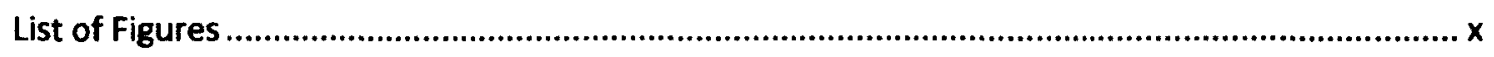

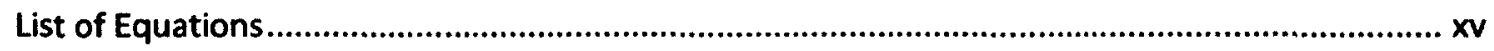

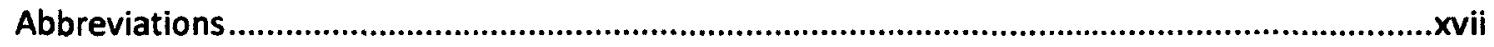

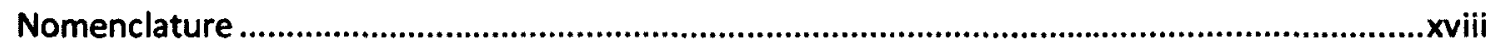

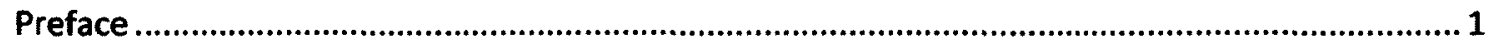

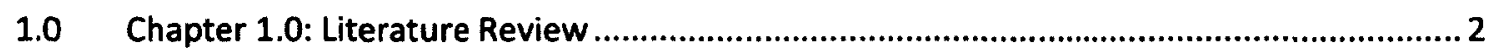

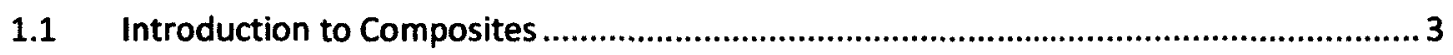

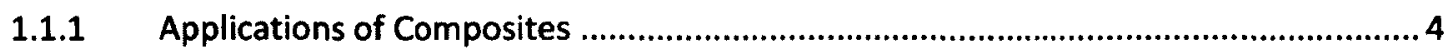

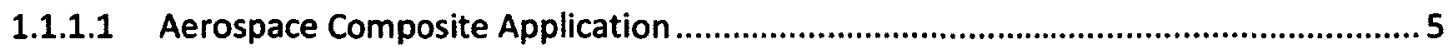

1.1.1.2 Other Composite Applications ...................................................................................

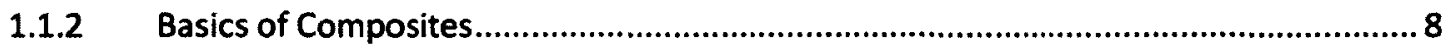

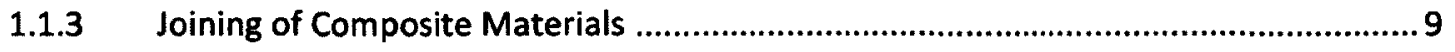

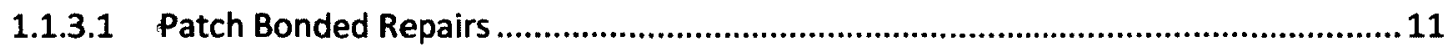

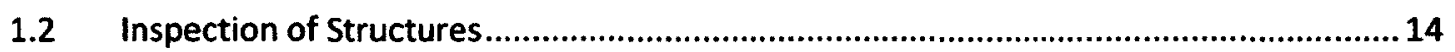

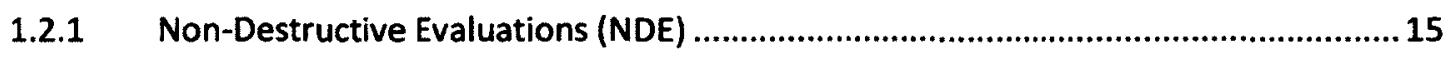

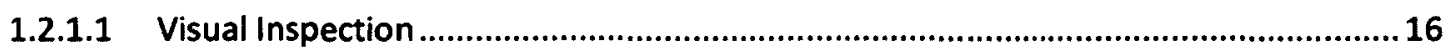

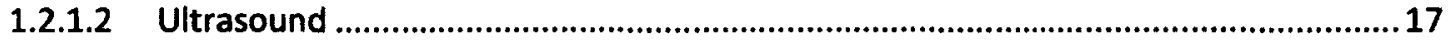

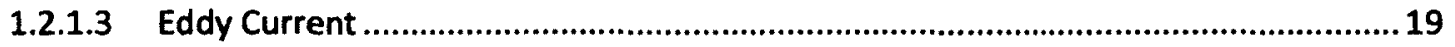

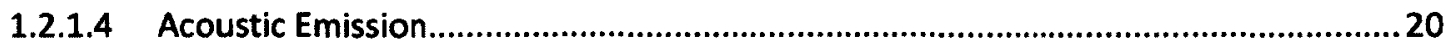

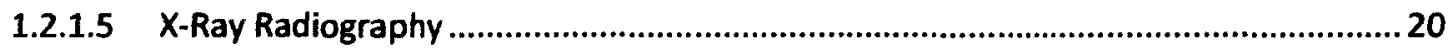

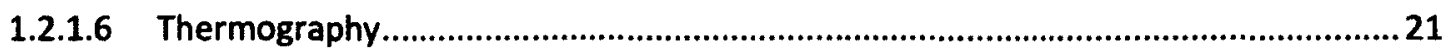

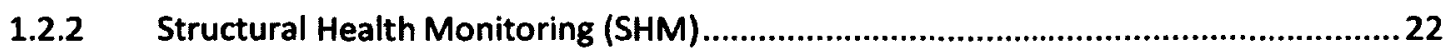

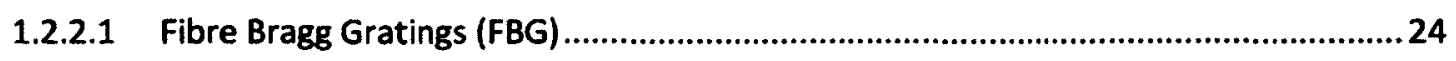

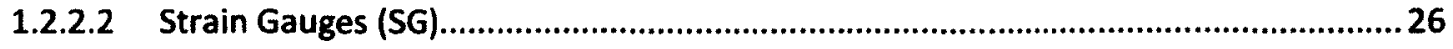

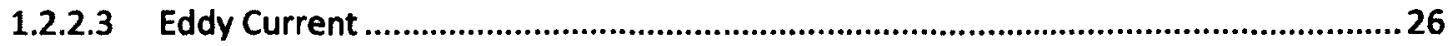

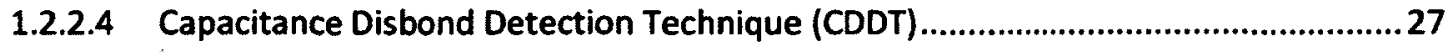




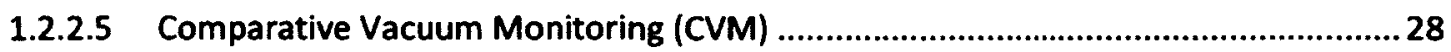

1.2.2.6 Surface Mountable Crack Detection System (SMCDS) .........................................29

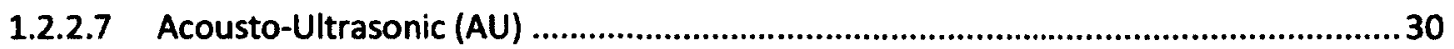

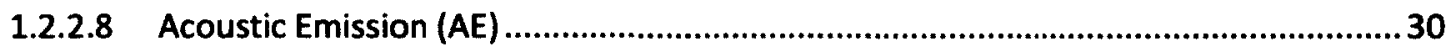

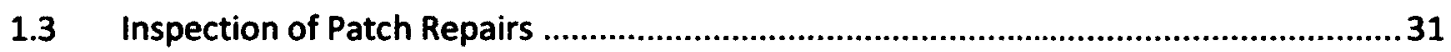

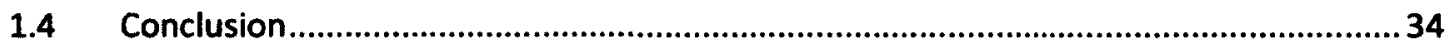

2.0 Chapter 2.0: Selection of Damage Detection Systems ......................................................36

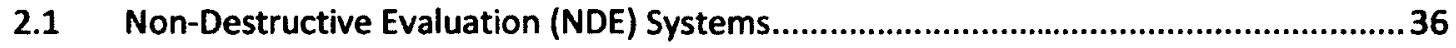

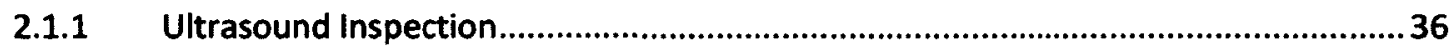

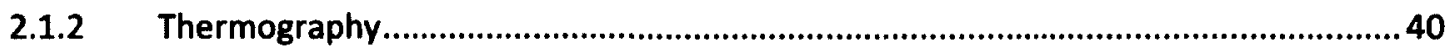

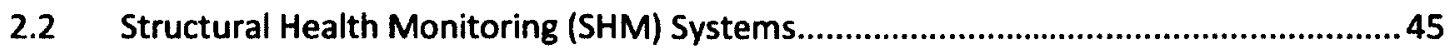

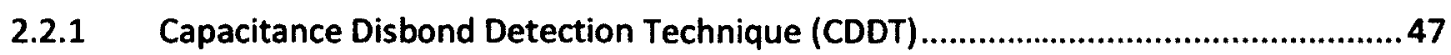

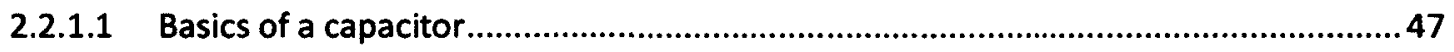

2.2.2 Surface Mountable Crack Detection System (SMCDS) ...............................................49

2.2.2.1 Basics of Surface Mountable Crack Sensors (SMCS) ................................................50

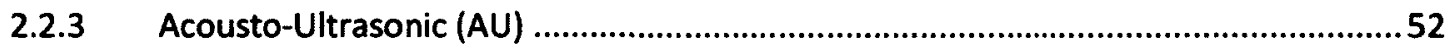

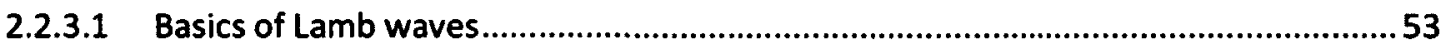

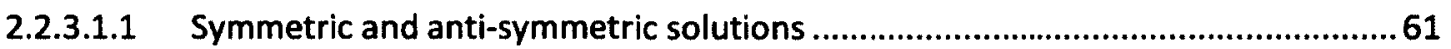

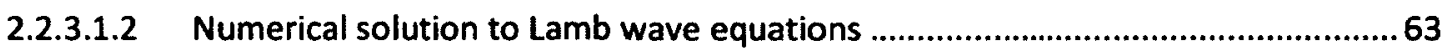

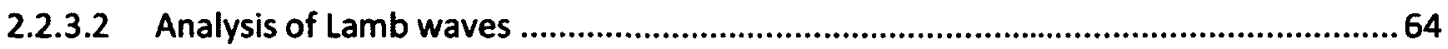

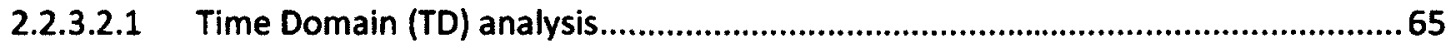

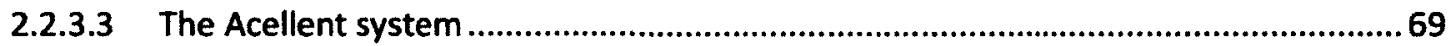

2.2.3.3.1 SmartPatch ${ }^{\bullet}$ application setup.......................................................................... 70

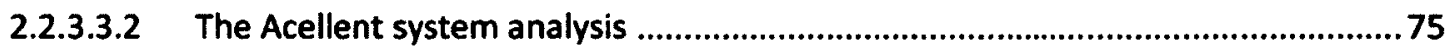

3.0 Chapter 3.0: Test Design and Coupon Manufacturing................................................... 78

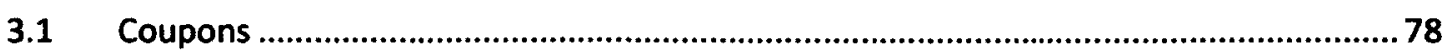

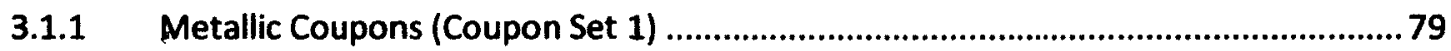

3.1.2 Patched Metallic Coupons (Coupon Set 2) .................................................................. 81

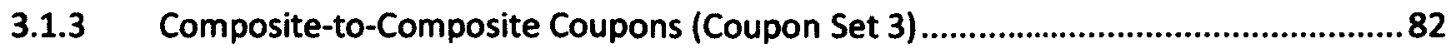

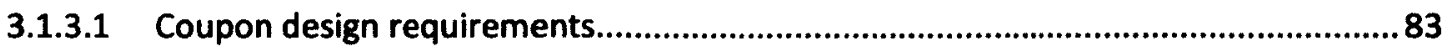

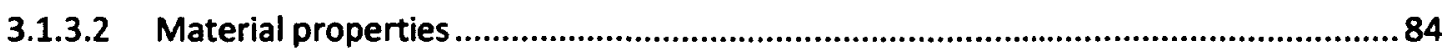

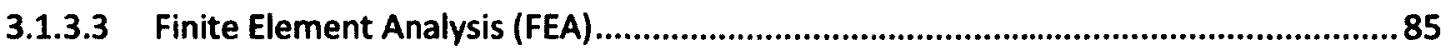




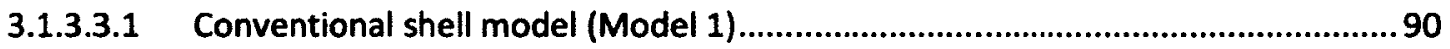

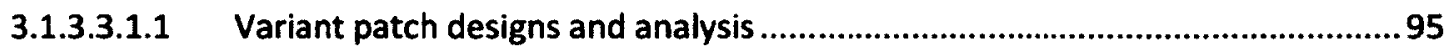

3.1.3.3.1.2 Variant patch geometry and analysis...........................................................96

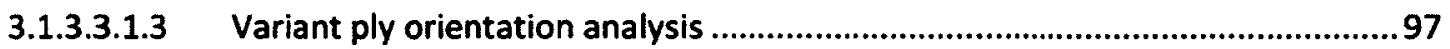

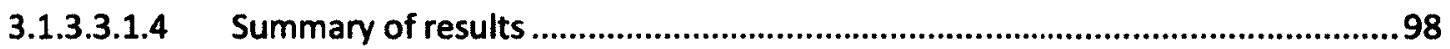

3.1.3.3.2 Continuum shell model (Model 2) .............................................................................99

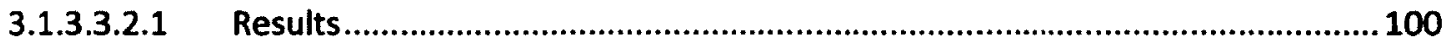

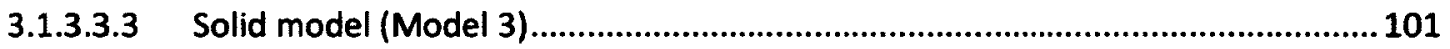

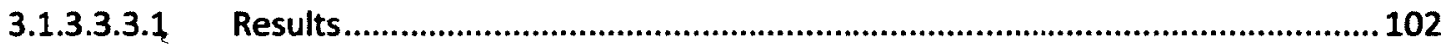

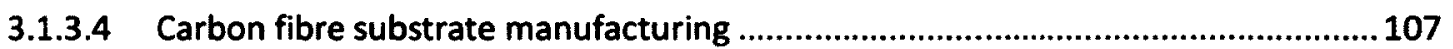

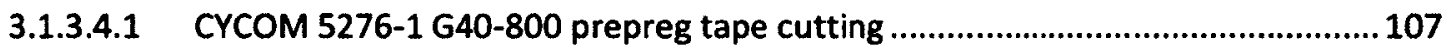

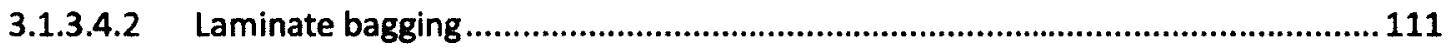

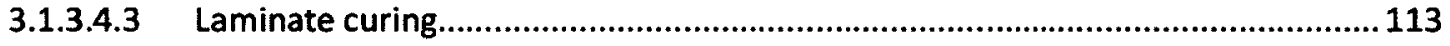

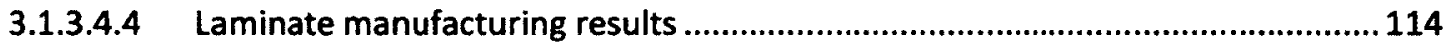

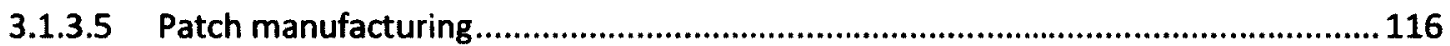

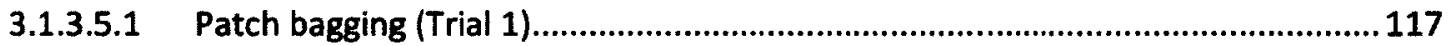

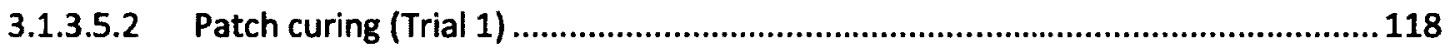

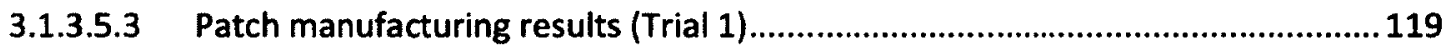

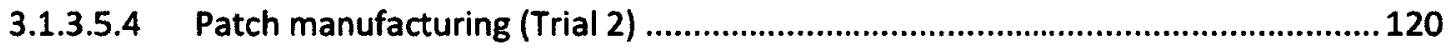

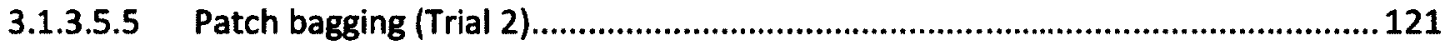

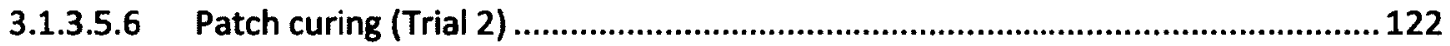

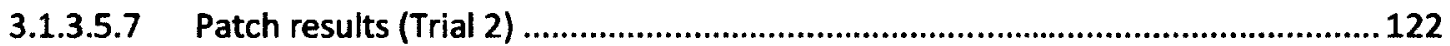

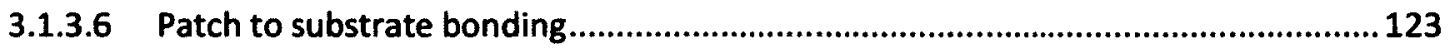

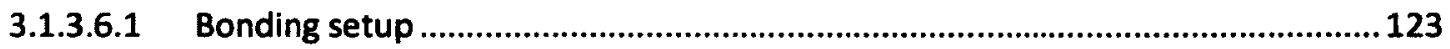

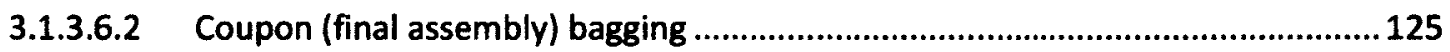

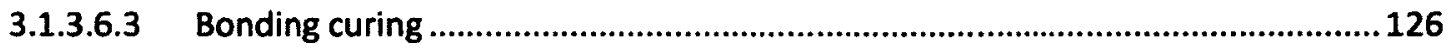

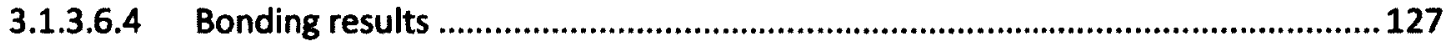

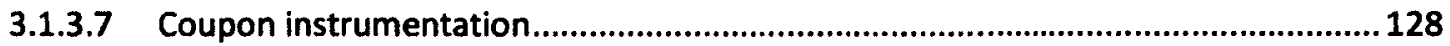

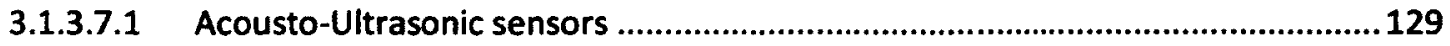

3.1.3.7.2 Capacitance Disbond Detection Technique (CDDT) sensors................................ 131

3.1.3.7.3 Surface Mountable Crack Detection System (SMCDS) ....................................... 132

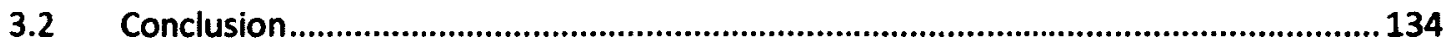




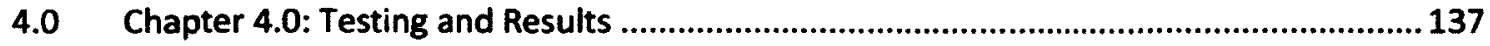

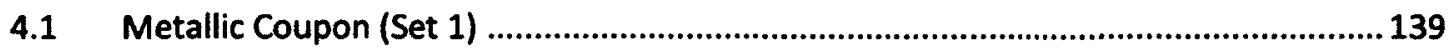

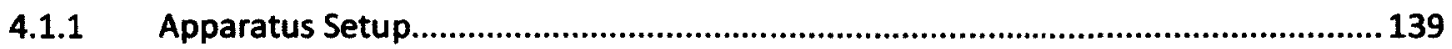

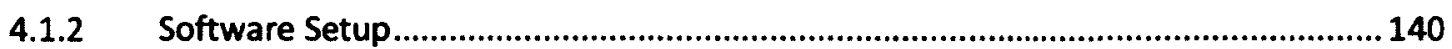

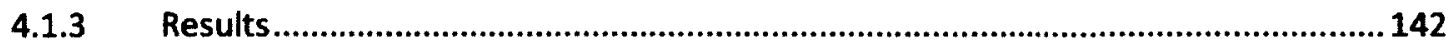

4.1.3.1 Direct Path Image (DPI) utility results ...................................................................... 143

4.1.3.2 Semi Empirical Damage Sizing (SEDS) results ..................................................... 146

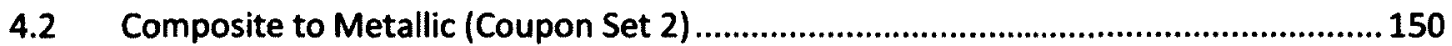

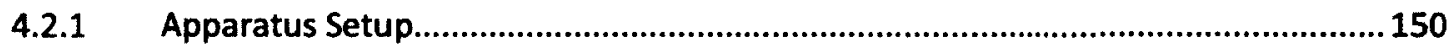

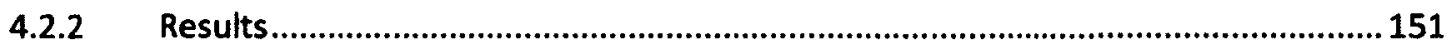

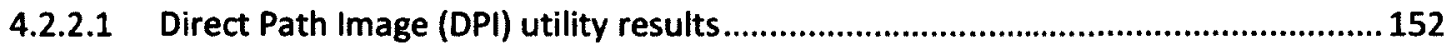

4.2.2.2 Semi Empirical Damage Sizing (SEDS) Results ....................................................... 162

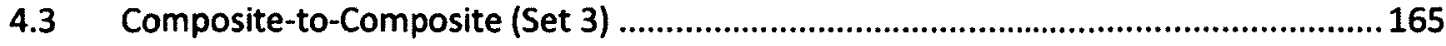

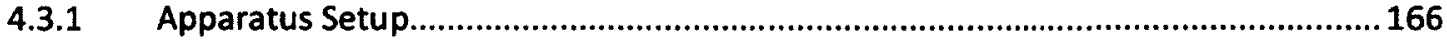

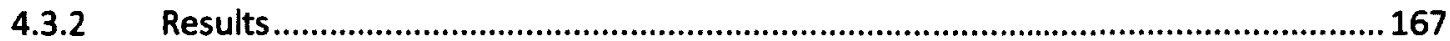

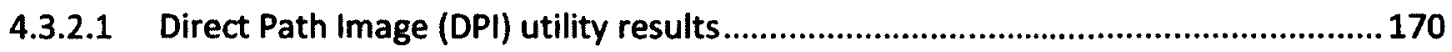

4.3.2.2 Semi Empirical Damage Sizing (SEDS) results ...................................................... 173

4.3.2.3 Capacitance Disbond Detection Technique (CDDT) results ....................................177

4.3.2.4 Surface Mountable Crack Detection System (SMCDS) results.................................181

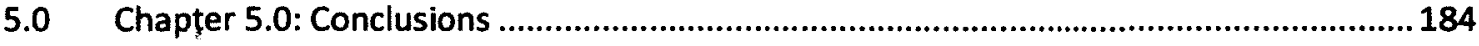

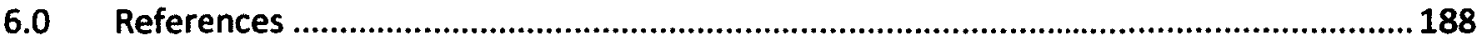




\section{List of Tables}

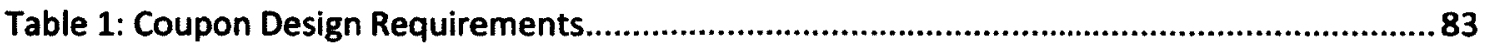

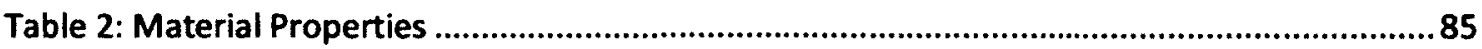

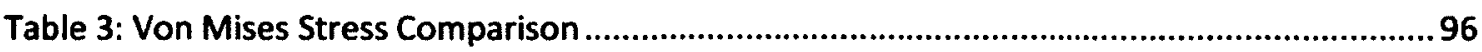

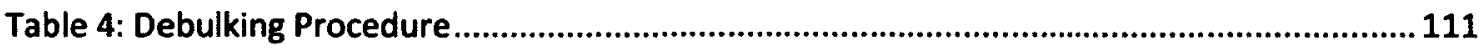

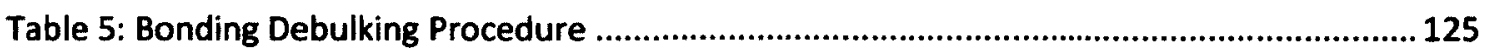




\section{List of Figures}

Figure 1: Fully Articulated Helicopter Rotor Hub: (a) All-Composite Hub; (b) Metallic Hub [9]...... 4

Figure 2: Utilization of Composites across the United States [10]

4

Figure 3: Composites Utilization within Military Application (Northrop Grumman B-2 bomber)

[11]

Figure 4: Composites Utilization within Commercial Application (Airbus A320) [11] ..................6

Figure 5: Composites Utilization within Combat Aircraft [11] ................................................. 7

Figure 6: Automotive Composites: (a) Aston Martin One-77 [14]; (b) Aston Martin One-77 Cross-

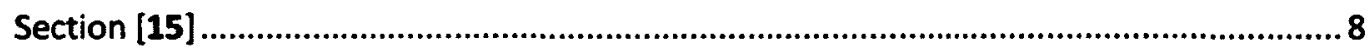

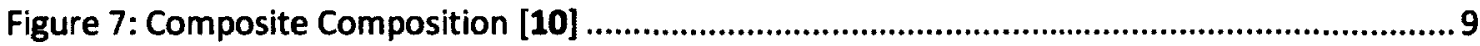

Figure 8: Composite Lay-up: (a) Continuous Fibres. (b) Discontinuous Fibres, and (c) Particle

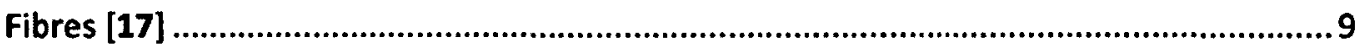

Figure 9: Australian Mirage III Crack Nucleation Locations [23] ............................................12

Figure 10: Australian Mirage III Patched Crack: (a) Repair Schematic and (b) Actual Repaired

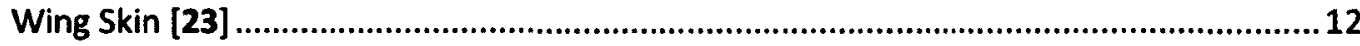

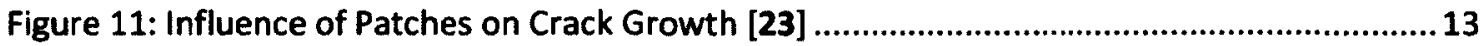

Figure 12: National Research Council Canada Holistic Structural Integrity Program ...................15

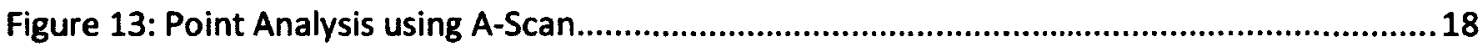

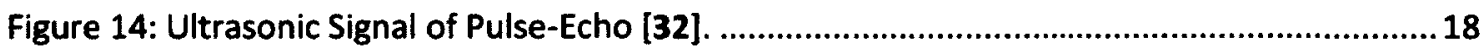

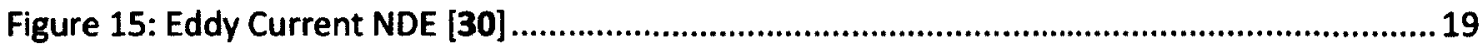

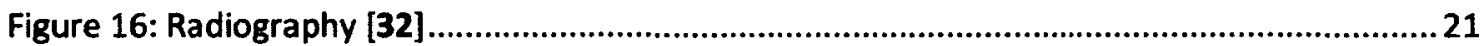

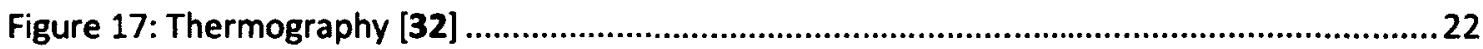

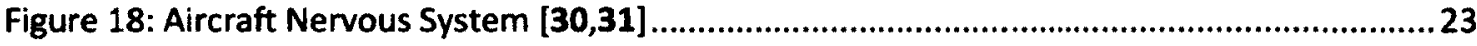

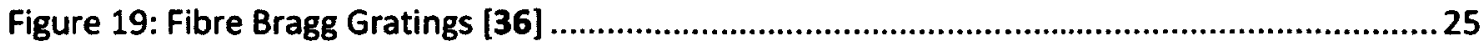

Figure 20: Strain Gauge and Circuit: (a) Typical Strain Gauge; (b) Typical Quarter Bridge Circuit

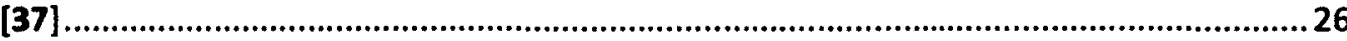

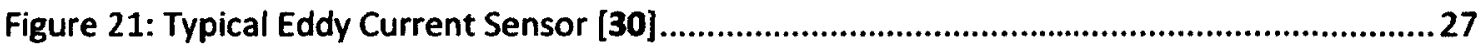

Figure 22: Capacitance Disbond Detection Technique: (a) Bonded Component; (b) Disbonded

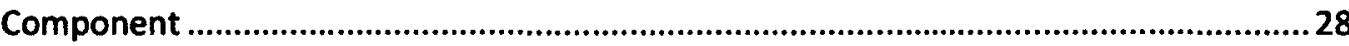

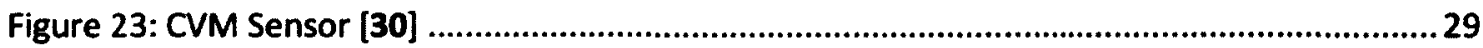

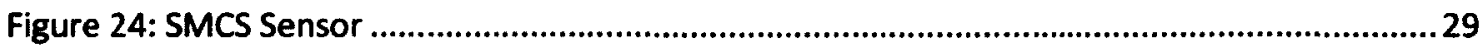

Figure 25: Acousto-Ultrasonic: (a) Sensor, Upper Side; (b) Sensor, Lower Side (Bond Surface); (c)

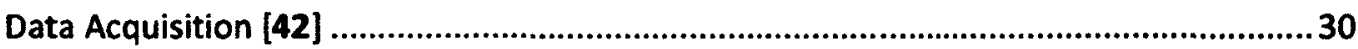

Figure 26: Simulated Patched Coupons: left displays a non-cracked coupon while the right is a

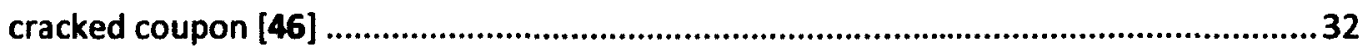

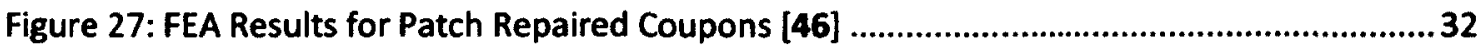

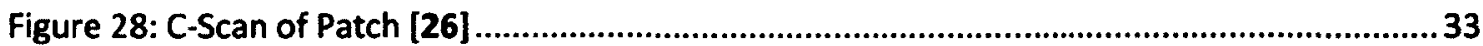

Figure 29: Ultrasonic Inspection using Pulse-Echo: (a) Component with Flaw and (b) Signal

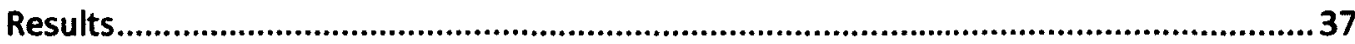

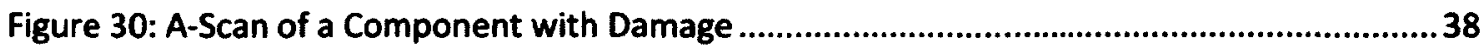

Figure 31: B-Scan of a Component with Damage ........................................................................39

Structural Health Monitoring of Adhesively Bonded Composite Joints Page $x$ 


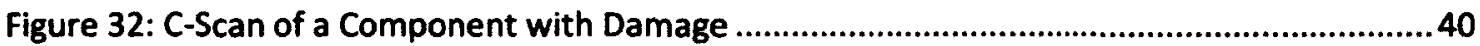

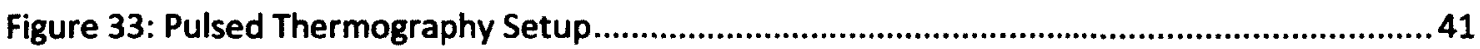

Figure 34: Pulsed Thermography Wave Propagation ........................................................41

figure 35: Heat Wave Propagation: (a) Pulsed Thermography, and (b) Lock-in Thermography [54]

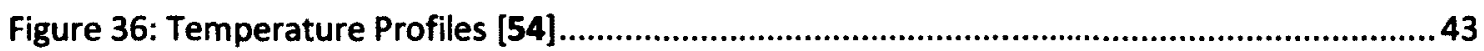

Figure 37: Example Thermography Images: (a) surface Thermal image after settling of temperature, and (b) Thermal image with uneven heating on [54].

Figure 38: Pulsed Thermography: (a) Pulse within time domain, and (b) Pulse within frequency domain [54]. . .45

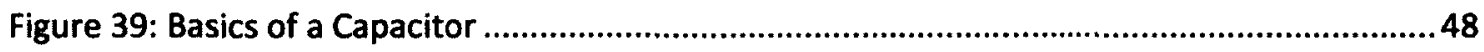

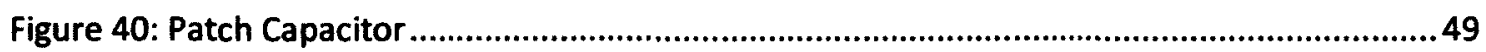

Figure 41: SMCS Circuits: (a) Intact SMCS acting as a closed switch and (b) cracked SMCS acting

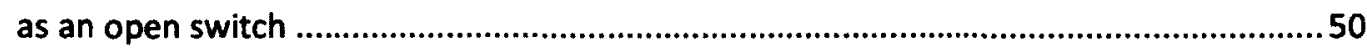

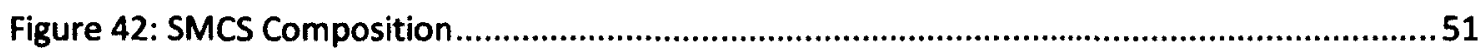

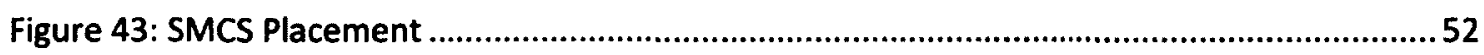

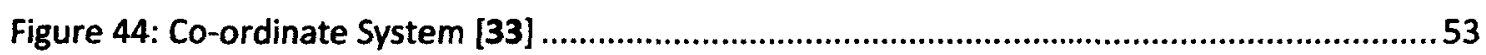

Figure 45: Particle Motion within a Solid Medium: (a) particles subject to pressure force, and (b)

particles subject to shear force [64] ...............................................................................5

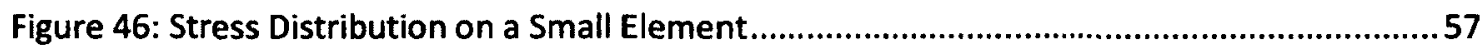

Figure 47: Lamb Wave Propagation: (a) Symmetric mode and (b) Anti-symmetric mode ...........61

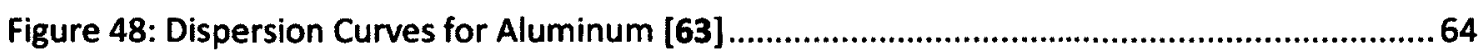

Figure 49: Lamb wave analysis of an aluminum plate: (a) Lamb waves and (b) Hilbert transform

[32]. .66

Figure 50: Correlation of benchmark and damaged plate: (a) Lamb waves signal of a benchmark composite plate, (b) Lamb waves of a damaged composite plate, (c) correlation coefficient plot, and $(d)$ the ratio between both correlation coefficients [32] ................67

Figure 51: Signal Scatter due to the Presence of Damage [32] ...................................................69

Figure 52: Acellent Sensor Setup: (a) sensor creation and (b) sensor setup ..............................71

Figure 53: Smart Layers: (a) single layer and (b) two smart layers............................................71

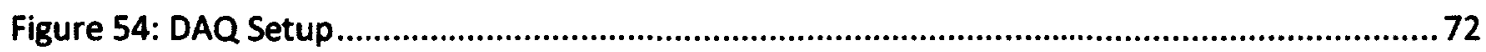

Figure 55: Path Definition: (a) path definition management, (b) path initiation and properties

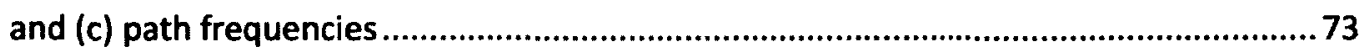

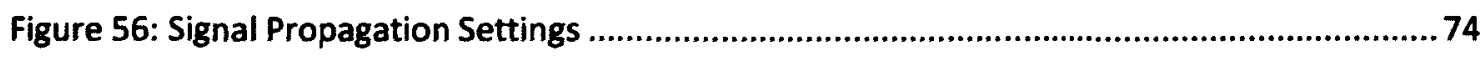

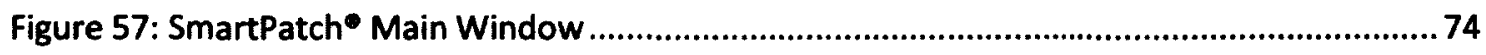

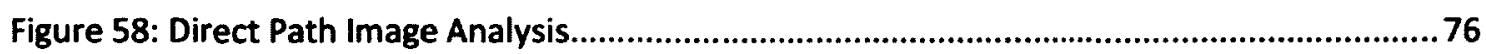

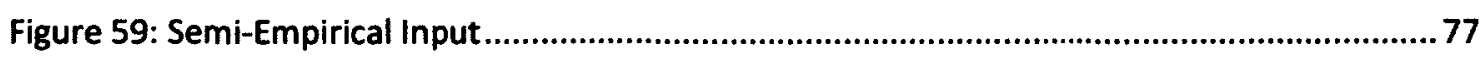

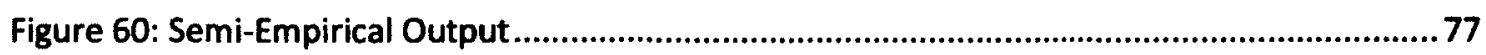

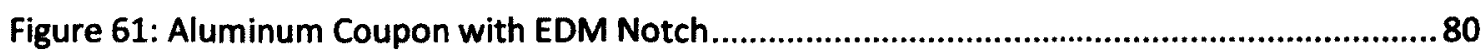

Figure 62: Aluminum Coupon Crack Growth: (a) Fatigued coupon with rib, (b) Fatigued coupon removed rib and (c) SMCS instrumented coupon prior to rib removal ...........................80

Figure 63: Smart Patch Composite-Metallic Coupon Design: (a) Substrate and (b) Patch............ 82

Structural Health Monitoring of Adhesively Bonded Composite Joints Page xi 
Figure 64: Common Stress Node

Figure 65: Conventional Shell Element Mesh Dependence Analysis........................................... 88

Figure 66: Continuum Shell Element Mesh Dependence Analysis ..........................................89

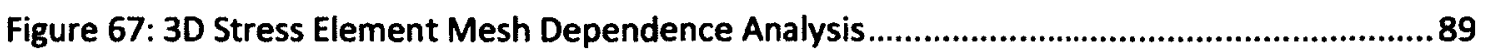

Figure 68: Smart Patch Phase 1 \& 2 Coupon Design (measurements in inches) ...........................90

Figure 69: Patch Part: (a) Spike Patch, (b) Chamfer Patch and (c) Unsymmetrical Spike Patch.... 91

Figure 70: Adhesive Part: (a) Adhesive for Patch 1, (b) Adhesive for Patch 2 and (c) Adhesive for

Patch 3

Figure 71: Substrate Part: (a) Dissections to Accommodate Patch 1, (b) Dissections to

Accommodate Patch 2 and (c) Dissections to Accommodate Patch 3 ...........................93

Figure 72: Assembly Position Constraints: (a) Isotropic View and (b) Side, Exploded View .........94

Figure 73: Loads and Boundary Conditions: (a) Model 1, (b) Model 2 and (c) Model 3............... 95

Figure 74: Von Mises Adhesive Results: (a) Spike Patch, (b) Chamfer Patch and (c) Unsymmetrical

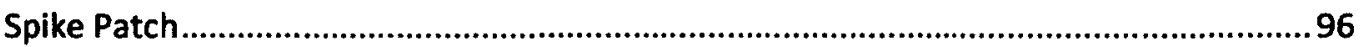

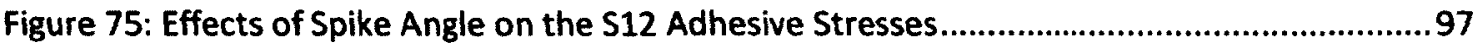

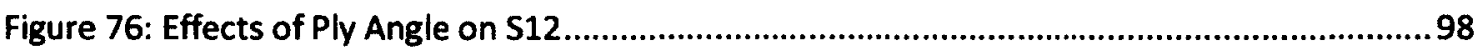

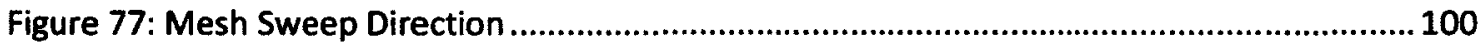

Figure 78: Comparison of both Continuum and Conventional Shell Models ............................. 101

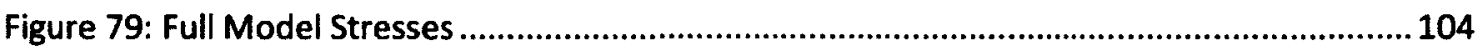

Figure 80: Stresses Distribution: (a) Patch, (b) Adhesive and (c) Magnified Adhesive ................ 105

Figure 81: Change in Stress over Application of Heat and Load ........................................... 106

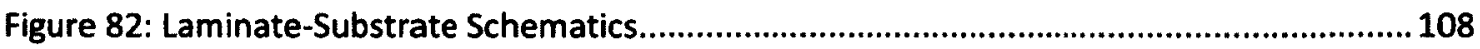

Figure 83: Ply Cutting Procedure: (a) Carbon Fibre Roll, (b) $45^{\circ}$ Alignment, (c) Straight Edge, and

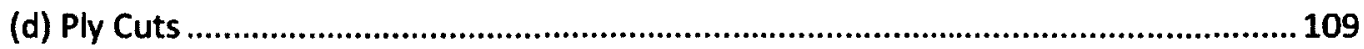

Figure 84: Layup Procedure: (a) Layup, and (b) Entrapped Air Removal................................... 110

Figure 85: Layup Bagging: (a) Laminate Placement, (b) Covering of Laminate, (c) Breather Cloth,

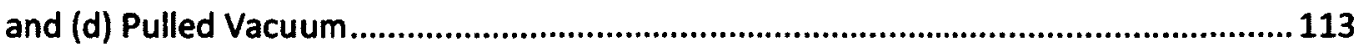

Figure 86: CYCOM 5276-1 Temperature and Pressure Profiles .............................................114

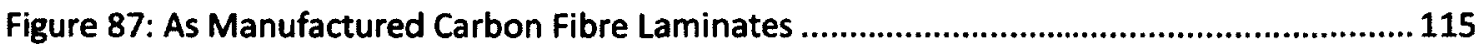

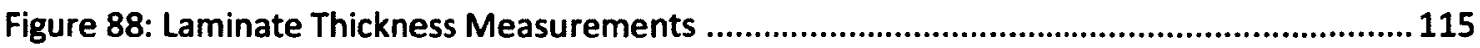

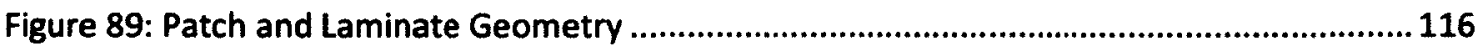

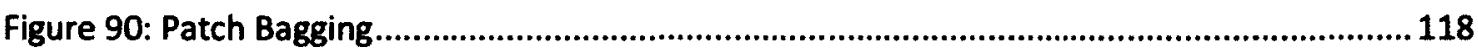

Figure 91: Specialty Materials 5521F Boron Prepreg Tape Temperature and Pressure Profiles 119

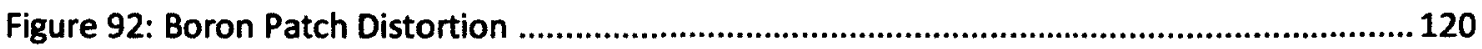

Figure 93: Patch Manufacturing Trial 2: (a) Patches with Tape Edge Dams, (b) Patches with

Surrounding Peel Ply ........................................................................................................... 121

Figure 94: Patch Bagging Trial 2: (a) Patches with Tape Edge Dams, (b) Patches with Surrounding

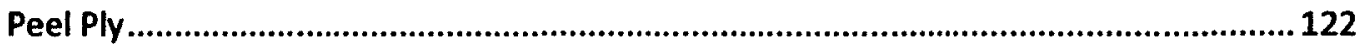

Figure 95: Patch Results Trial 2: (a) Patch with Tape Edge Dams and Surrounding Shims, (b) Patch with Tape Edge Dams and No Surrounding Shims, (c) Patch with Surrounding Peel Ply and Surrounding Shims and (d) Patch with Surrounding Peel Ply and No Surrounding Shims. 
Figure 96: Bonding Procedure: (a) Surface Preparation, (b) Patch to Substrate Alignment and (c)

Entrapped Air Removal. 124

Figure 97: Bonding Bagging: (a) Coupon Wrapping, (b) Coupon Placement (c) Applying Vacuum 126

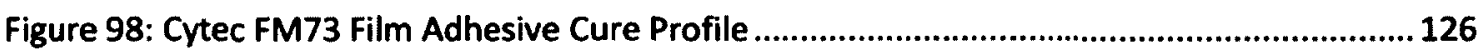

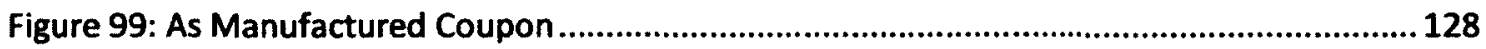

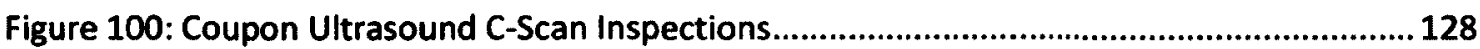

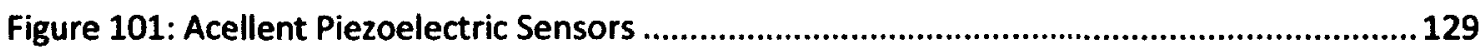

Figure 102: Piezoelectric Sensors Layout: (a) Sensor layout 1, (b) Sensor layout 2 and (c) Sensor

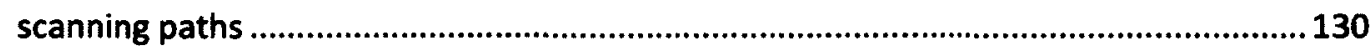

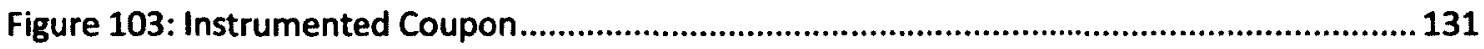

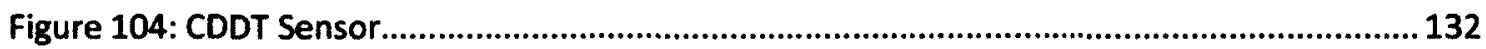

Figure 105: SMCDS: (a) insulating layer installation, (b) Insulating layer, (c) conductive layer

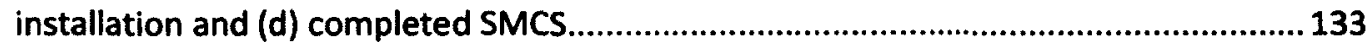

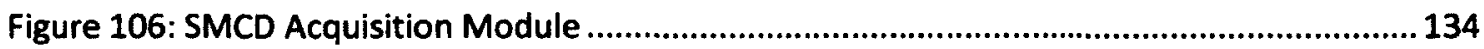

Figure 107: Coupon Sailures: (a) Coupon Set 1 Fracture and (b) coupon Set 2 disbond and

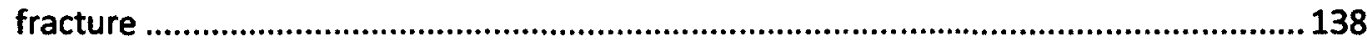

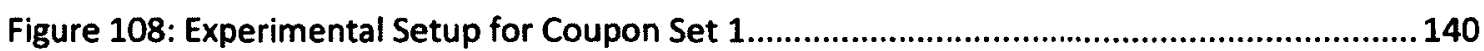

Figure 109: Coupon: (a) Actual coupon and (b) SmartPatch ${ }^{\star}$ coupon digital mapping

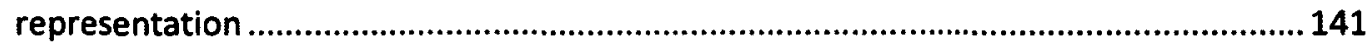

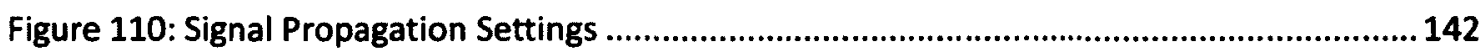

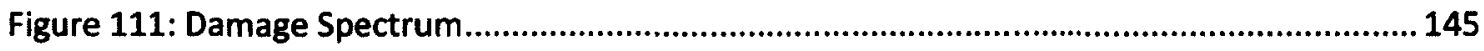

Figure 112: Coupon SPP2-12 Direct Path Image Results at a 0.5 Threshold .............................145

Figure 113: Coupon SPP2-15 Direct Path Image Results at a 0.5 Threshold .............................. 145

Figure 114: Semi-Empirical Interpretations for Coupon SPP2-12 .........................................148

Figure 115: Semi-Empirical Interpretations for Coupon SPP2-15 ......................................... 148

Figure 116: Semi-Empirical Interpretations for Coupon SPP2-26........................................... 149

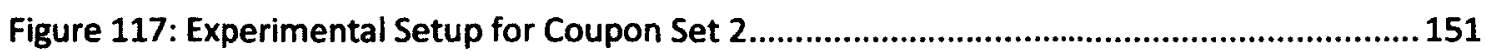

figure 118: Estimated Disbond Areas using Thermography....................................................152

Figure 119: SPP2-11 Scanning Zones: (a) Zone 1, (b) Zone 2 and (c) Zone 3............................ 153

Figure 120: Coupon SPP2-11: (a) DPI for all zones at a 0.5 threshold, (b) DPI for zone 1 at a 0.5

threshold, (c) DPI for zone 2 at a 0.5 threshold and (d) Thermography images ............. 155

Figure 121: Coupon Scanning Zones: (a) SPP2-13 zones, (b) SPP2-14 and SPP2-18 scanning zones

Figure 122: Coupon SPP2-13: (a) DPI for all zones at a 0.5 threshold, (b) DPI for zone 1 at a 0.5 threshold, (c) DPI for zone $2 \& 3$ at a 0.5 threshold, (d) DPI for zone 4 at a 0.5 threshold and $(e)$ Thermography images..............................................................................158

Figure 123: Coupon SPP2-14: (a) DPI for all zones, (b) DPI for zone 1, (c) DPI for zone 2 and (d)

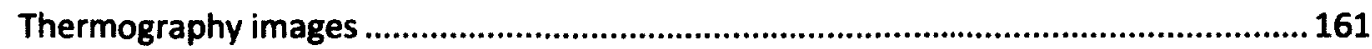

Figure 124: Coupon SPP2-11 Semi-Empirical Interpretations .................................................. 163

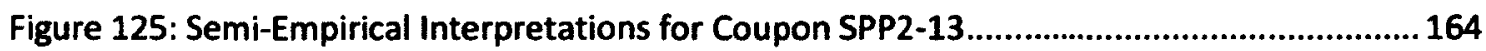

Figure 126: Semi-Empirical Interpretations for Coupon SPP2-14 ............................................ 165

Structural Health Monitoring of Adhesively Bonded Composite Joints Page xiii 
Figure 127: Disbond Area Estimations: (a) Coupon, (b) Adhesive cracking and (c) Estimated area

Figure 128: Sensor disbond

Figure 129: Signal Change due to Sensor Sisbond: (a) Signal path and (b) Signal change due to

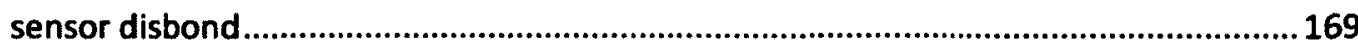

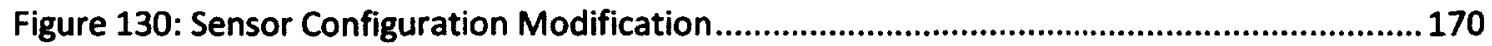

Figure 131: Coupon SPP3-04: (a) DPI utility results at 05 threshold and (b) Estimated disbond

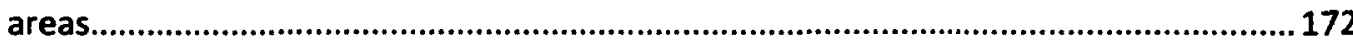

Figure 132: Coupon SPP3-12: (a) DPI utility results at 0.5 threshold and (b) Estimated disbond

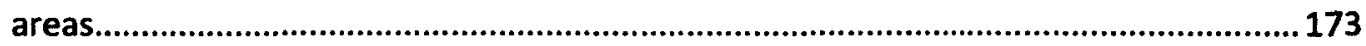

Figure 133: Semi-Empirical Interpretations for Coupon SPP3-04.............................................. 175

Figure 134: Semi-Empirical Interpretations for Coupon SPP3-4a ..............................................176

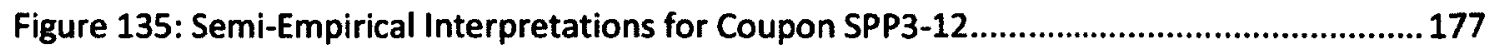

Figure 136: SPP3-16 Disbond Area Comparison to Capacitance Drop .......................................178

Figure 137: SPP3-12 Disbond Area Comparison to Capacitance Drop ........................................179

Figure 138: SPP3-16 Normalized Disbond Area Comparison to Capacitance Drop ......................180

Figure 139: SPP3-12 Normalized Disbond Area Comparison to Capacitance Drop......................180

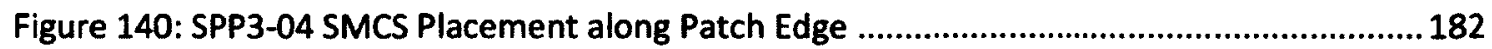

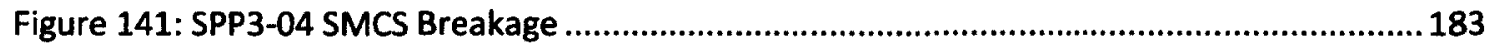

Figure 142: SPP3-4a SMCS Breakage at High Cycles.................................................................. 183 


\section{List of Equations}

Equation 1

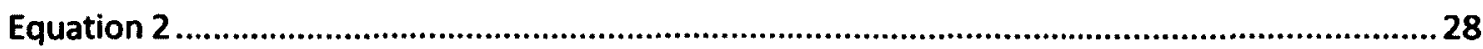

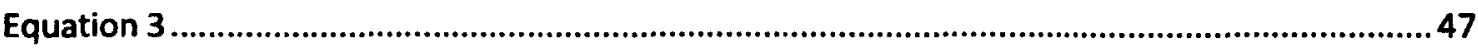

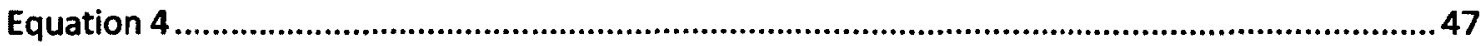

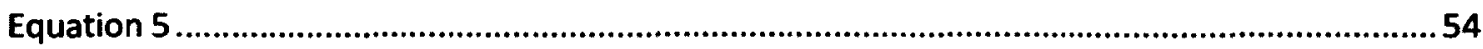

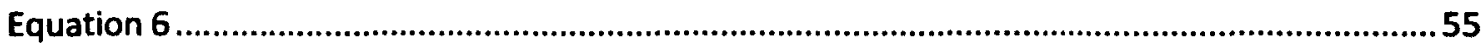

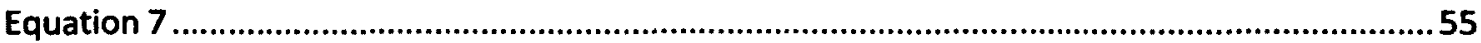

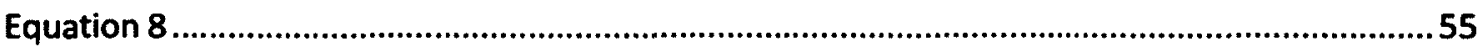

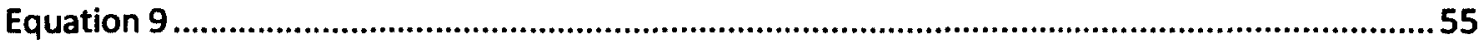

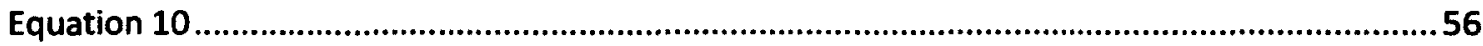

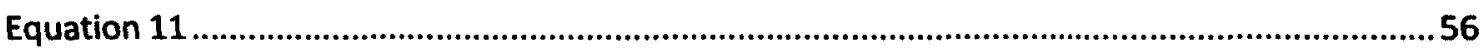

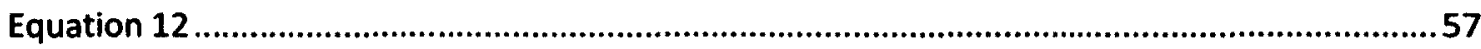

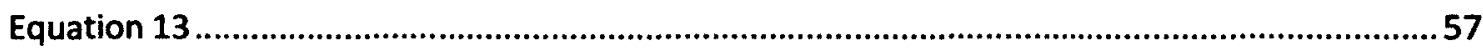

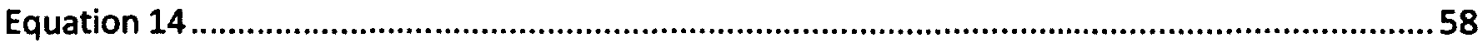

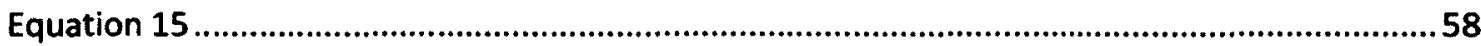

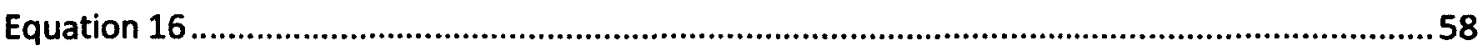

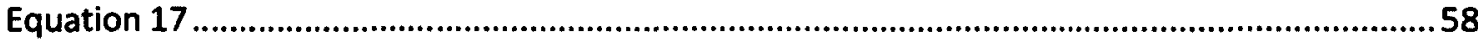

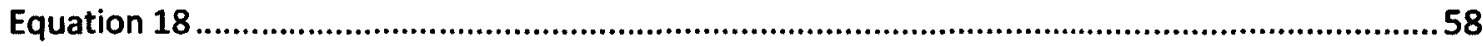

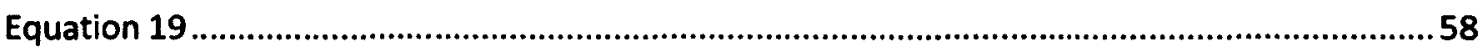

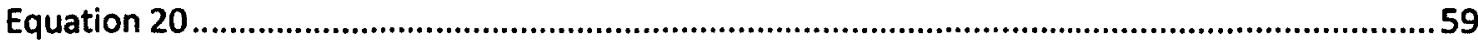

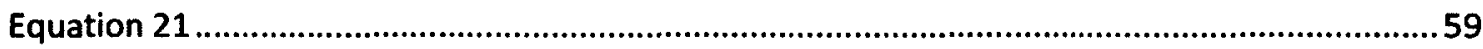

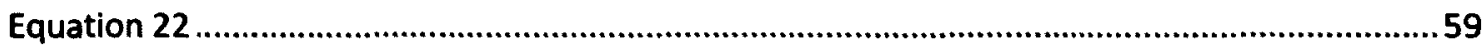

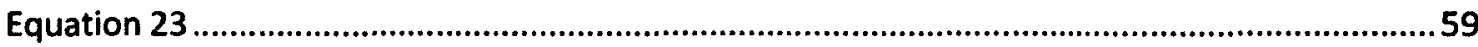

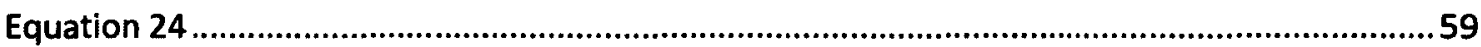

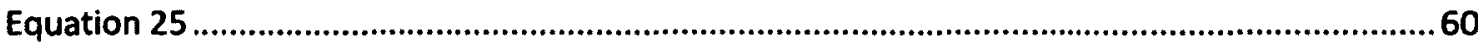

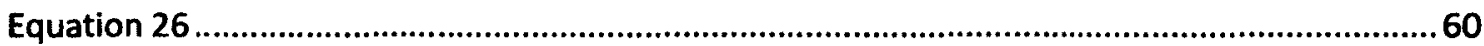

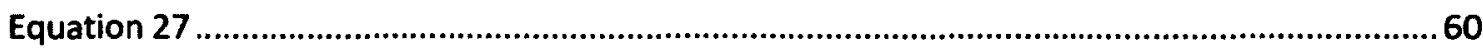

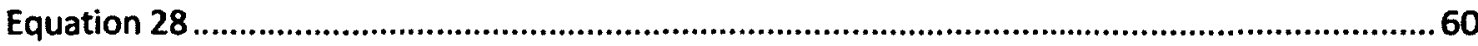

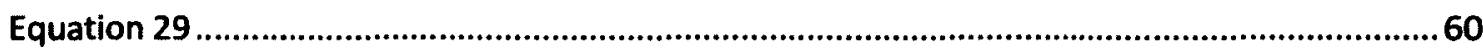

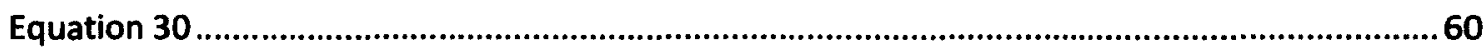

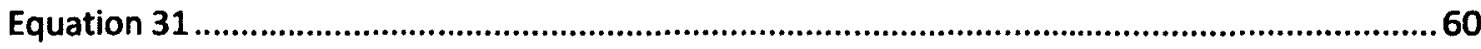

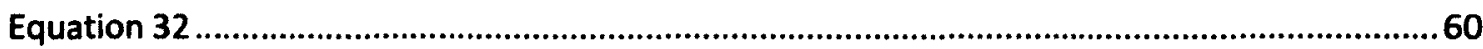

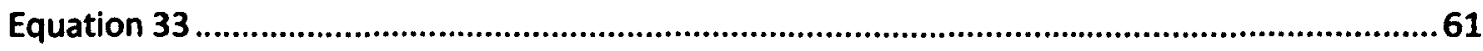

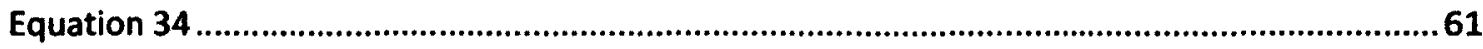

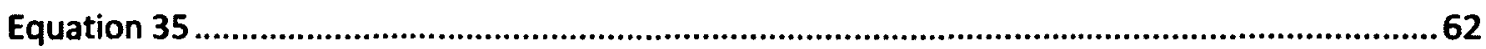

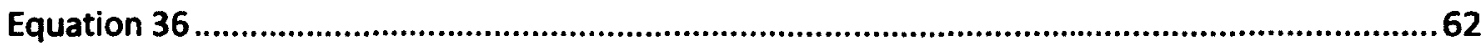

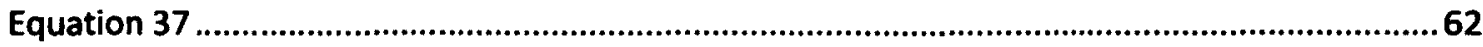

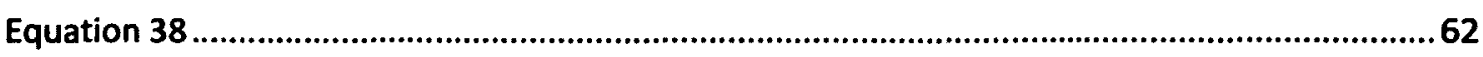

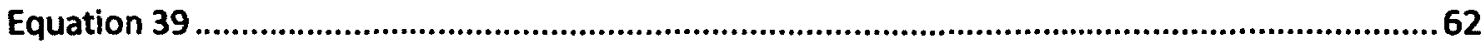

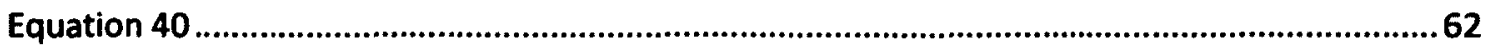




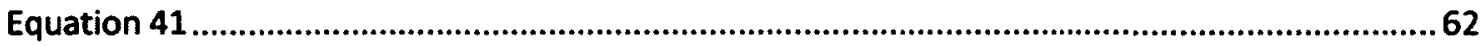

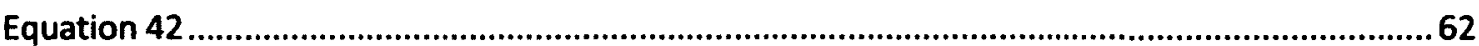

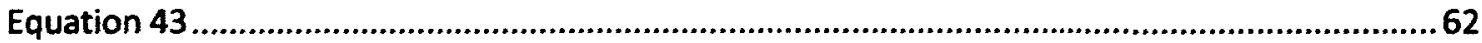

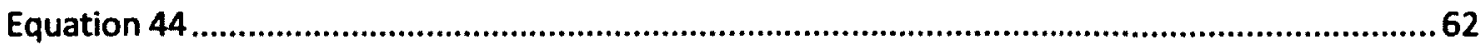

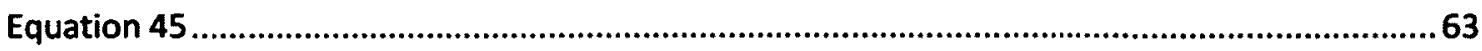

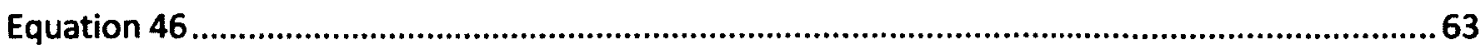

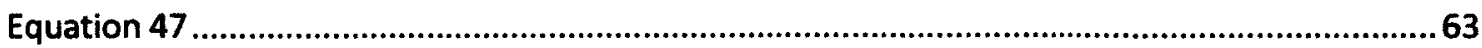

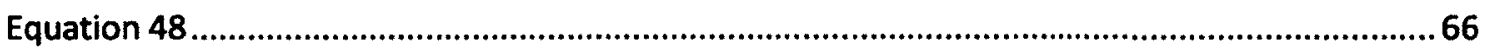

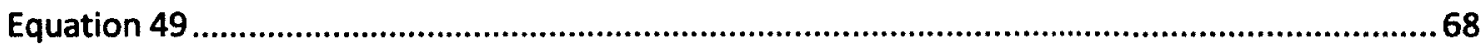




\section{Abbreviations}

\begin{tabular}{|l|l|}
\hline Abbreviation & Description \\
\hline AE & Acoustic Emission \\
\hline AFGROW & Air Force Growth \\
\hline AU & Acousto-Ultrasonic \\
\hline CDDT & Capacitance Disbond Detection Technique \\
\hline CFRP & Carbon Fibre Reinforced Polymer \\
\hline CVM & Comparative Vacuum Monitoring \\
\hline DAQ & Data Acquisition \\
\hline DFT & Discrete Fourier Transforms \\
\hline DI & Damage Index \\
\hline DPI & Direct Path Image \\
\hline DSP & Digital Signal Processing \\
\hline FBG & Fibre Bragg Grating \\
\hline FEA & Finite Element Analysis \\
\hline LT & Lock in Thermography \\
\hline LW & Lamb Wave \\
\hline MTS & Material Test Frame \\
\hline NDE & Non-Destructive Evaluation \\
\hline NRC & National Research Council Canada \\
\hline PANTA & Phosphoric Acid Non-Tank Anodizing \\
\hline PPT & Pulsed Phase Thermography \\
\hline PT & Pulsed Thermography \\
\hline PZT & Lead Zirconate Titanate \\
\hline RAAF & Royal Australian Air Force \\
\hline SEDS & Semi-Empirical Damage Sizing \\
\hline SG & Strain Gauge \\
\hline SHM & Structural Health Monitoring \\
\hline SMCDS & Surface Mountable Crack Detection System \\
\hline SMCS & Surface Mountable Crack Sensor \\
\hline TD & Time Domain \\
\hline ToF & Time of Flight \\
\hline UT & Ultrasound Inspection \\
\hline
\end{tabular}




\section{Nomenclature}

\begin{tabular}{|c|c|}
\hline Symbols & Description \\
\hline$\lambda_{\mu_{w}}$ & Reflected wave number \\
\hline$\Lambda$ & Spacing between grits within an FBG \\
\hline $\bar{c}$ & Capacitance \\
\hline $\bar{K}$ & Dielectric constant \\
\hline$\varepsilon_{0}$ & Permittivity of free space $\left(8.854 \times 10^{-12} \mathrm{~F} / \mathrm{m}\right)$ \\
\hline$d$ & Thickness \\
\hline $\boldsymbol{v}$ & Voltage \\
\hline$\underline{n}$ & Average refractive index \\
\hline$F$ & External or body force \\
\hline$m$ & Mass \\
\hline$a$ & Acceleration \\
\hline$\sigma$ & Normal stress \\
\hline$\tau$ & Shear stress \\
\hline$T$ & Stress tensor \\
\hline$\varepsilon$ & Direct strain \\
\hline$\lambda$ & Lame constant \\
\hline$G$ & Shear modulus \\
\hline$E$ & Young's modulus \\
\hline $\boldsymbol{v}$ & Poisson's ratio \\
\hline$A$ & Cross-sectional area \\
\hline$u$ & Displacement in $\mathrm{x}$-direction \\
\hline $\boldsymbol{v}$ & Displacement in y-direction \\
\hline $\boldsymbol{w}$ & Displacement in $\mathrm{z}$-direction \\
\hline$C_{L}$ & Longitudinal wave speed in elastic solid \\
\hline$C_{T}$ & Transverse wave speed in elastic solid \\
\hline$t$ & Time \\
\hline$\rho$ & Density \\
\hline$\gamma$ & Shear strain \\
\hline$u$ & Displacement \\
\hline $\overrightarrow{\vec{e}}$ & Unit vector \\
\hline$e$ & Exponential \\
\hline$\nabla$ & Gradient or divergence \\
\hline$\phi$ & Scalar potential \\
\hline$\psi$ & Vector potential \\
\hline$i$ & Imaginary number \\
\hline$\omega$ & Angular frequency \\
\hline$A_{1}$ & Constant of integration \\
\hline$A_{2}$ & Constant of integration \\
\hline$B_{1}$ & Constant of integration \\
\hline$B_{2}$ & Constant of integration \\
\hline$k$ & Circular wave number \\
\hline$C_{g}$ & Group velocity \\
\hline$C_{p}$ & Phase velocity \\
\hline$\lambda_{x y}$ & Correlation Coefficient \\
\hline$A p$ & Signal amplitude \\
\hline
\end{tabular}




\section{Preface}

Aviation advancements have been on the rise since the Wright brothers took the first ever powered flight in late 1903. Since that remarkable day, military aircraft have far advanced to incorporate both agility and stealth, while civilian aircraft have advanced in both efficiency and safety. In recent years, both civilian and military requirements have been increasingly satisfied through the use of advanced materials such as composites. However, composite materials have many uncertainties as it relates to aging under operational environments. Unlike traditional metallic materials, composites are susceptible to many forms of damage that are not visible to the naked eye. These damages are a safety concern when left unrepaired, especially as aircraft age.

Many in-service civilian and military aircraft today have well exceeded their design life. However, airlines have made economic decisions to maintain and overhaul these aircraft rather than purchasing new models for their fleet. Aging aircraft as well as new aircraft must be either inspected by a mechanic or by an automated system. Inspections by aircraft mechanics require removal of the aircraft from service which results in loss of profit for the airline. Automated inspection systems may be a solution to eliminating the need to remove the aircraft from service. These automated inspection systems are known as Structural Health Monitoring (SHM) systems. SHM systems may be used on composite and metallic materials to determine the initiation and growth of damage.

This research is focused on the evaluation of SHM systems for their effectiveness in damage detection and quantification of failure within both metallic-to-composite and composite-tocomposite adhesive joints. 


\section{Chapter 1.0: Literature Review}

Composite materials have a variety of applications ranging from fashionable rings to turbine fan blades. These materials are composed of reinforcing fibres and resin matrix. Fibres provide the load carrying capability while the resin acts as a load transfer medium. Various combinations of these two materials allow for different strength and therefore make composites very tailorable for specific applications. Tailoring of composites does not end at component curing, rather at the time an assembly is complete. Assemblies composed of two or more composite components may be joined through the use of adhesives. Adhesives provide an optimal stress distribution as compared to mechanical fasteners. However, unlike mechanical fasteners, adhesive joints are not easily inspectable as the joint does not allow for disassembly.

The integrity of adhesive joints may be inspected through routine maintenance using NonDestructive Evaluations (NDE) or through the use of Structural Health Monitoring (SHM) systems. There are many SHM system technologies that exist today, many of which are sold as out-of-the-box solutions which are ready for integration and interrogation of structural components. However, not all of these systems are effective and only a few are currently under evaluation for certification on commercial aircraft $[1,2]$. In this research, several SHM systems will be evaluated for the detection of crack growth and disbond.

This chapter provides necessary background required for understanding composites, adhesive joints, NDE and SHM technologies. 


\section{I.1 Introduction to Composites}

Current advancements in materials science have led to increased use of advanced materials in aircraft, automotive and civil structures. Advanced materials such as carbon fibre reinforced polymers (CFRP) are composed of 2 phases: the fibre (reinforcement) and the matrix (load transfer medium) $[3,4,5]$.

Within the aerospace industry, the wide use of composite materials has been driven by their mechanical, electrical, and thermal properties. However, wide use of composites is due to mechanical properties such as the specific strength and toughness $[4,5]$. Composite structures first appeared on commercial aircraft in the 1970 s as a direct result of the oil crisis. Composites were implemented in an attempt to reduce aircraft weight and therefore increase fuel efficiency. Components made of composite materials on commercial aircraft at the time included vertical and horizontal stabilizers. These advanced materials were then adopted in many other aircraft applications, one of which was helicopter blades. Unlike metallic structures, composite structures are heterogeneous and therefore stiffness in different directions can be controlled during manufacturing [5]; thus leading to the reduction in part count [6]. Figure 1(a) shows an example of a modern composite helicopter hub while Figure 1(b) shows an example of a traditional metallic helicopter hub. Both hubs serve the same purpose; however, a composite hub utilizes significantly lower part count and therefore is lighter and possesses a lower probability of failure. The reduction in part count is also a direct result of the reduction in mechanical fasteners; this is due to the wide use of adhesive joints [7]. However, even with the all the advantages, composite materials have not completely replaced traditional metallic components and that is due to their disadvantages which are later described in section 1.1.1.1 [8]. 


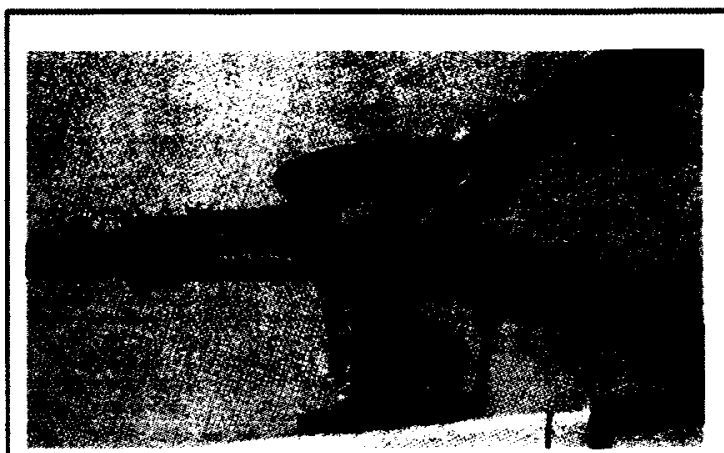

(a)

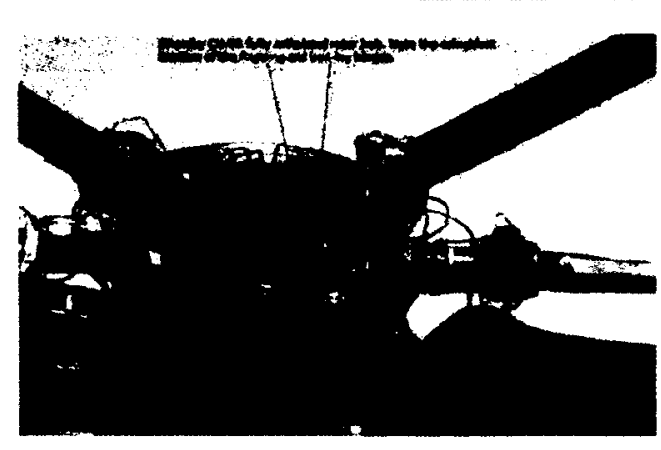

(b)

Figure 1: Fully Articulated Helicopter Rotor Hub: (a) All-Composite Hub; (b) Metallic Hub [9]

\subsubsection{Applications of Composites}

Even though this thesis primarily concentrates on aircraft structures, composite materials are more commonly used in other applications [10]. Within the United States, during the years 1999 to 2000 , the total amount of composite materials used for the aerospace industry accounted for approximately $0.8 \%$ of all shipped raw composites [10]. Figure 2 below shows the quantities of composites that were shipped to each of the respective industry sectors.

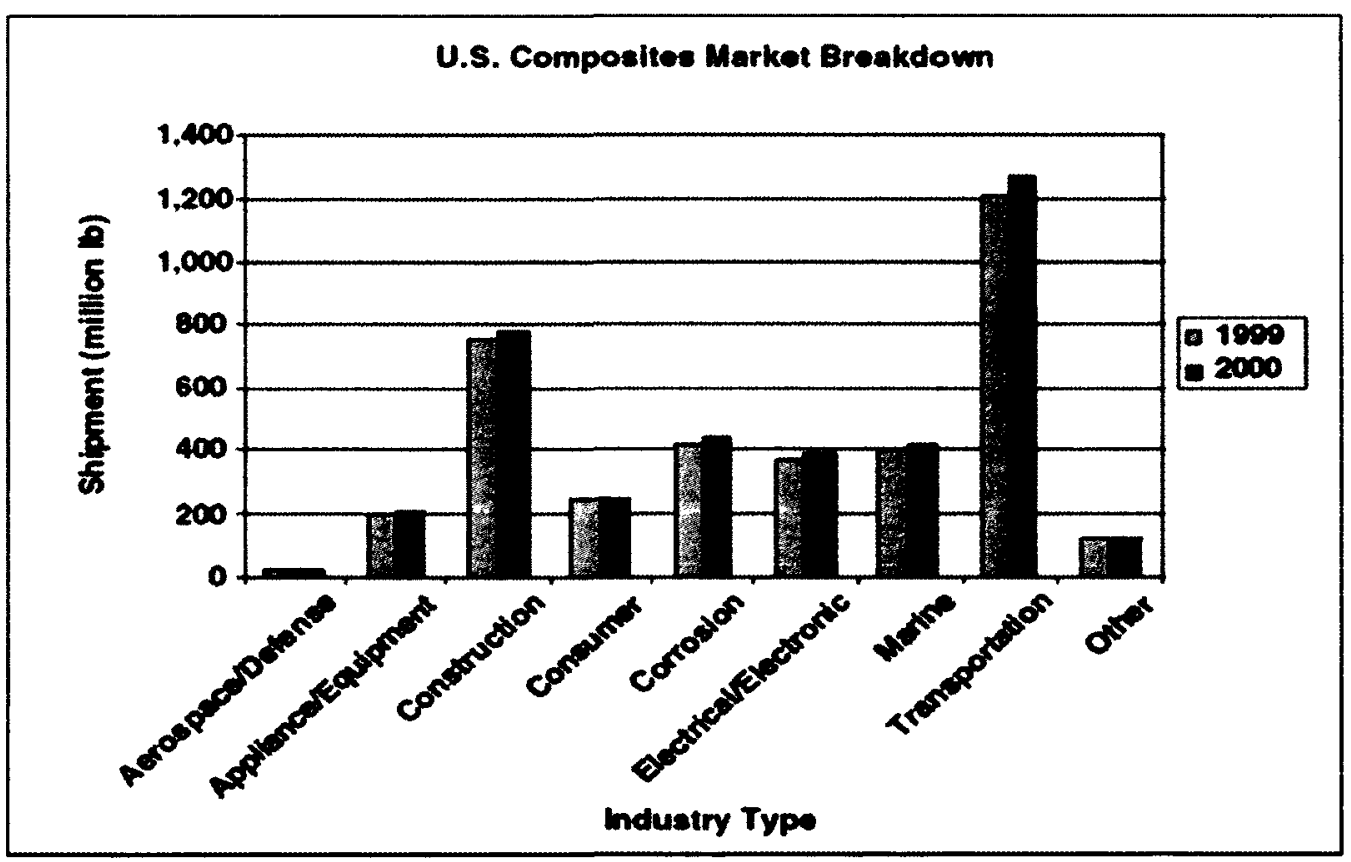

Figure 2: Utilization of Composites across the United States [10] 


\subsubsection{Aerospace Composite Application}

The United States Air Force was one of the first organizations to utilize composite materials in the aerospace/defence industry. Composite materials on fighters such as the F-111, F-14, F-15 and F-16 included such components as skins for the aerodynamic surfaces [10]. Introduction of composites (primarily boron based fibres) for use on fighters enabled the reduction of weight of the individual components by up to $35 \%$. This reduction in weight allowed fighters to carry heavier payload while increasing range $[8,10,11]$. After research into composites by the Air Force during the Cold War, the civilian aircraft industry began to slowly implement composite components as part of their structures [8]. Both Figure 3 and Figure 4 show composite components on military (Northrop Grumman B-2 Bomber) and civilian (Airbus A320) aircraft; it is clear that civilian aircraft utilize significantly less composite materials, a difference of approximately $40 \%$ of the overall structural weight [11]. The difference of composite percentage usage for both the A320 and the B-2 bomber are defined by their design requirements. Figure 5 shows a plot of composite usage percentage for many aircraft that have been produced with various flight requirements over a 40 year time span beginning in 1965. It is clear from Figure 5 that since the 1960s composite usage has been on the rise for both the military and civilian aerospace industries. Aircraft in the early 1970 s utilized less than $5 \%$ according to the graph while aircraft produced in the early 2000 s utilized $30 \%$ (or less) of composites within their structural weight. With the exception of the Beechcraft Starship and the Northrop Grumman B-2 bomber, all other aircraft structures are composed of no more than $30 \%$ composite; this was true until recent introduction of the Boeing B787 [11,12]. The Beechcraft Starship was the first of its kind to utilize significant amount of composite materials. However, due to the lack of understanding of composite materials' performance and aging, the Starship was later removed 
from service [13]. The Starship's early retirement was due to lack of understanding of 3D loads and the effects of the environment on the composite components [11].

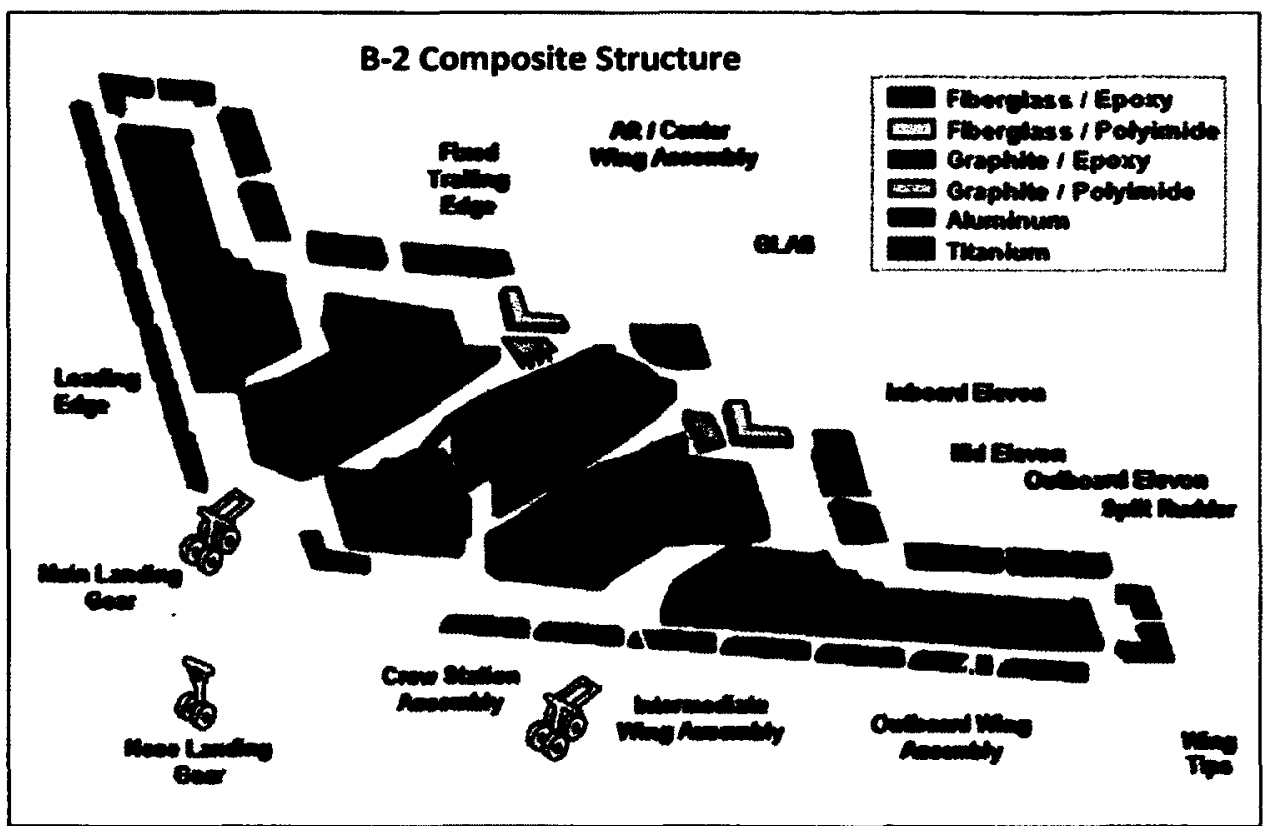

Figure 3: Composites Utilization within Military Application (Northrop Grumman B-2 bomber) [11]

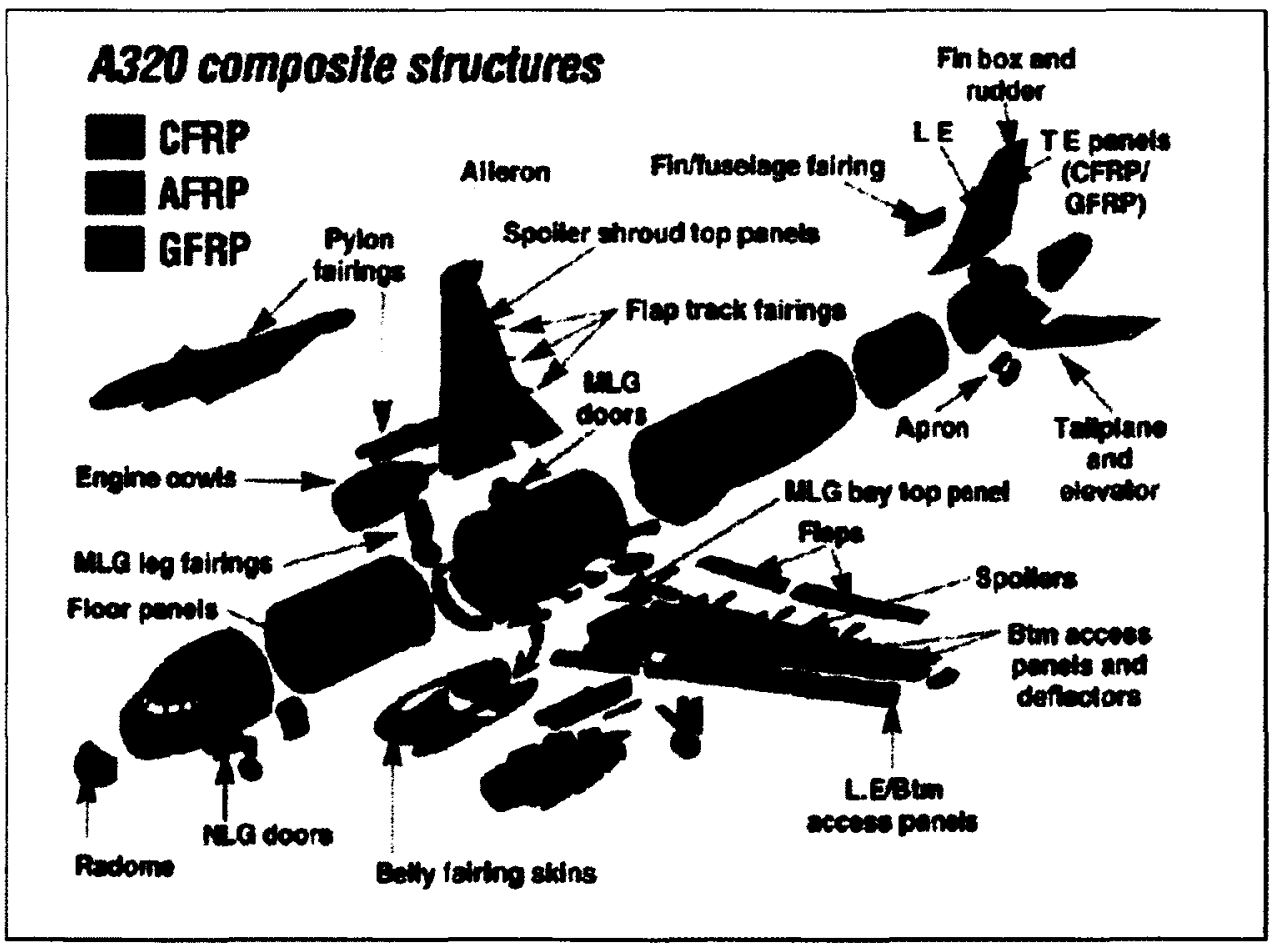

Figure 4: Composites Utilization within Commercial Application (Airbus A320) [11] 


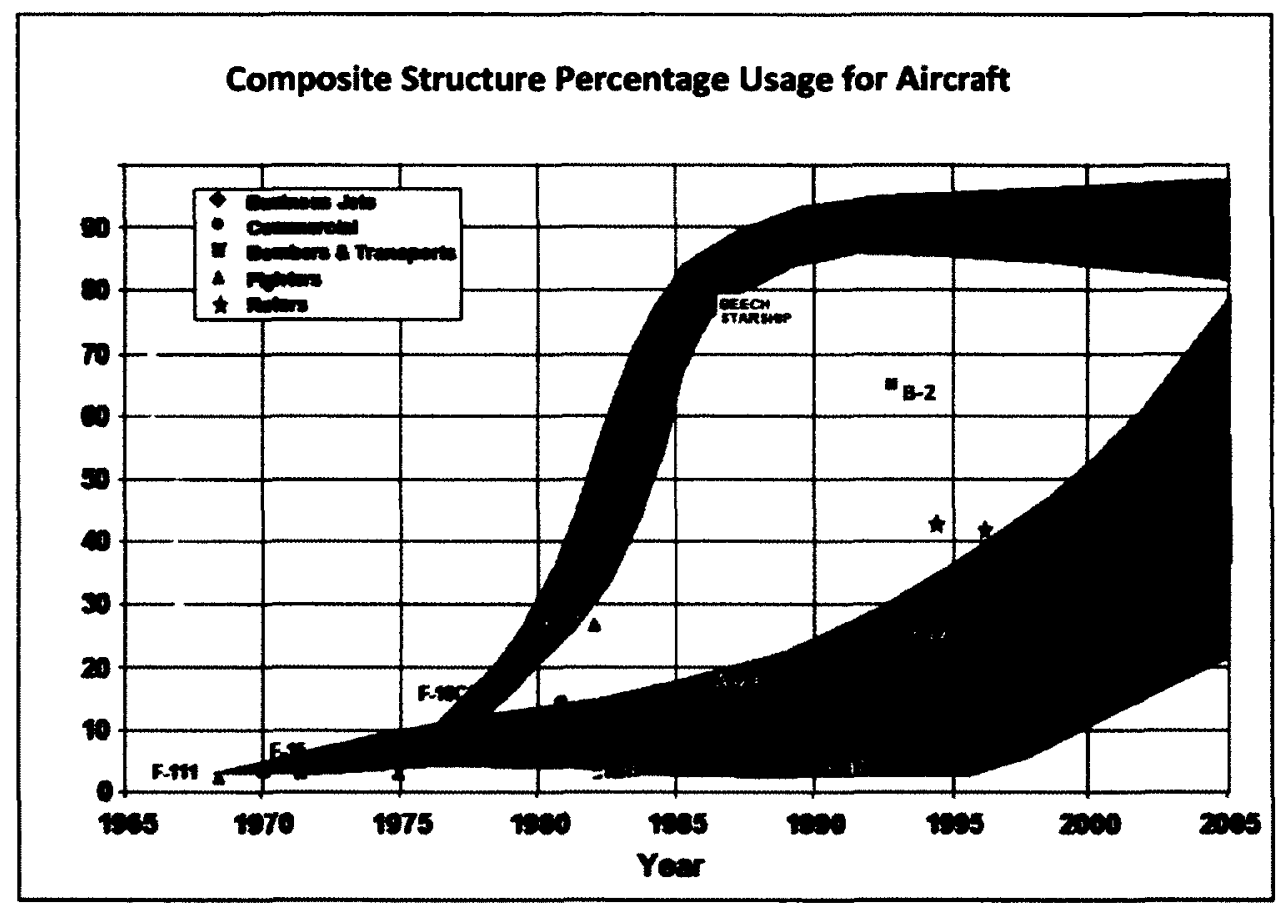

Figure 5: Composites Utilization within Combat Aircraft [11]

\subsubsection{Other Composite Applications}

Composite materials have been used for many other applications where weight influences the performance of the component and overall system efficiency.

The automotive industry consumes a large amount of composite materials on many types of vehicles ranging from high-end exotic performance cars to small trucks. Composite components are used in vehicles to replace non-structural components that would normally be made out of steel [10]. However, unlike the utilization of carbon fibre based composites within the aerospace industry, the automotive industry utilizes glass fibres. The choice of glass fibres, as opposed to carbon fibre, is driven by raw material cost. Glass fibres are cheaper and meet the strength requirements of the automotive industry [10]. Carbon fibre based composites are being implemented on the chassis and frames of high-end sports cars that cost more than $\$ 300,000$ USD [14,15]; such as the Aston Martin One-77 as shown in Figure 6. 


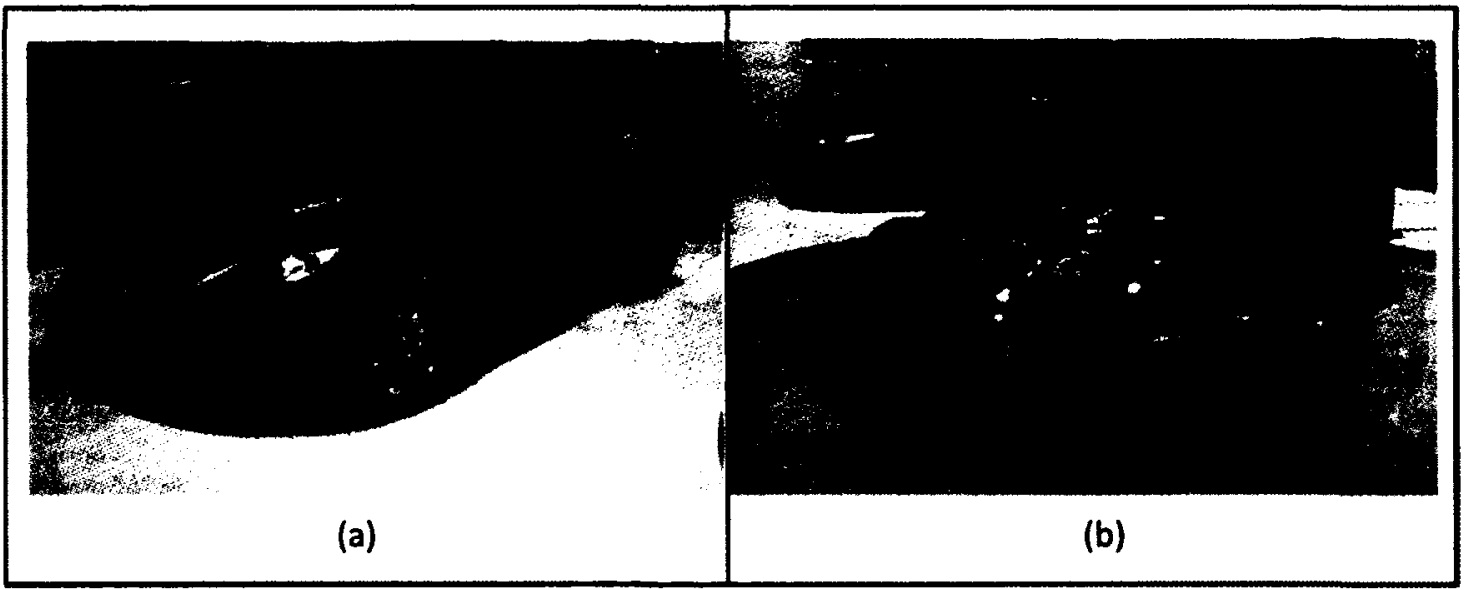

Figure 6: Automotive Composites: (a) Aston Martin One-77 [14]; (b) Aston Martin One-77 Cross-Section [15]

The sports industry also uses composites for sports products that require specific stiffness and directional flexibility; these include but are not limited to golf sticks, hockey sticks, tennis racquets and baseball bats. Through the selection of the proper fibres and fibre orientation, manufacturers can acquire the correct stiffness that would be tailored for each player's swing.

The construction industry also uses composite materials for bridge repair; these would include both glass fibre and carbon fibre doublers [10]. Also, within the construction industry, another form of composite material that is widely used is reinforced concrete [16]. In concrete, the reinforcing fibres are steel rods and the matrix is the concrete itself [16].

\subsubsection{Basics of Composites}

Composite components are composed of two or more materials to create a single entity/component. Of the two materials, one acts as the load carrying material while the other acts as the load transfer medium. The load carrying material is a fibre while the load transfer medium is a resin, as shown in Figure 7. Fibres can be of any material such as carbon, boron, glass, graphite, steel, etc. The resin is some form of plastic, usually a thermoset; however, in recent years, thermoplastics have been also used to make composite components. 


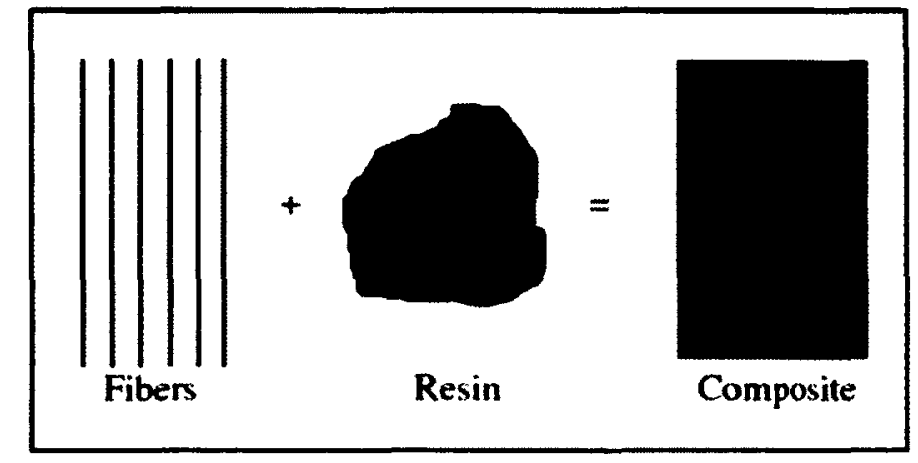

Figure 7: Composite Composition [10]

A single ply (sheet) of composite material is formed by combining fibre and resin as shown in

Figure 7. Multiple plies are stacked onto each other to form a larger and thicker composite component as shown in Figure $8(a)$. In addition to ply stacking, there exist other forms of composite material that do not use continuous fibres, both of which are shown in Figure $8(b)$ and Figure 8(c) [17]. Discontinuous fibres and partial fibres are chopped up from the same fibre strand as that used for ply stacking [17]. Chopped fibres allow for distributed strength in all directions and therefore allow the component to behave as an isotropic material. Because the resin is allowed to cure while the component is near its final shape, complex curvature may be obtained in a single step as compared to many required for alloys.

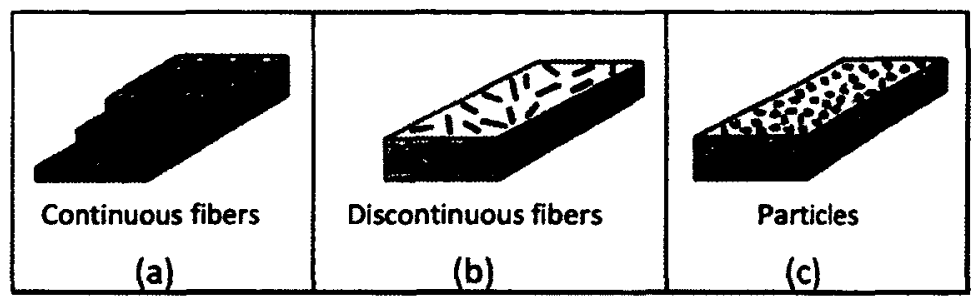

Figure 8: Composite Lay-up: (a) Continuous Fibres. (b) Discontinuous Fibres, and (c) Particle Fibres [17]

\subsubsection{Joining of Composite Materials}

Joining of composite materials may be done using mechanical fastening or adhesive bonding [18]. Both methods have their own advantages and disadvantages. In general, mechanical fasteners' advantages include: no surface preparation, easy disassembly and easy inspection 
while disadvantages include: stress concentration and increased component count. Bonding advantages include: smooth stress distribution and reduced component count while disadvantages include: difficult disassembly (in some cases impossible), difficult inspection and strict surface preparation requirements $[8,18,19]$. It may appear that bonded joint disadvantages outnumber the advantages; however, when evaluated, both weight reduction and smooth stress distribution overcome these disadvantages [20].

Adhesive bonding in the aerospace industry first appeared on the De Havilland Mosquito in the 1940s [21]. The Mosquito was built of plywood, balsa and spruce components. All of these components were bonded using adhesives. During World War II, bombers were manufactured using the aid of adhesives. However, aircraft during the war had an expected life of 600 hours prior to being shot during combat. Full understanding of strength and limitations of adhesive joints were not of concern as they were known to have a life greater than that of the bomber's expected life. Aircraft which since have survived the war and have ever since been in storage, have not shown any signs of major degradation as they have not been exposed to harsh weather conditions as compared to current in-service aircraft.

Current in-service aircraft are exposed to harsh conditions and high loads. Non-composite military in-service aircraft remain operational for more than $\mathbf{5 0}$ years while some non-composite commercial aircraft such as $B 747$ have spent more than 30 years in-service prior to retirement. As these aircraft age in the presence of harsh environments and loads, components crack and adhesive joints degrade which affect the safety and performance of the aircraft. To prolong the life of components, composite patches may be bonded in areas of cracks to redistribute stresses. Also, composite joints may be used on new aircraft to eliminate fasteners which create stress concentrations that give rise for crack nucleation or composite delamination. 


\subsubsection{Patch Bonded Repairs}

During the 1980s, many aging in-service mirage fighter aircraft of the Royal Australian Air Force (RAAF) began to exhibit signs of cracking at the wing root skin as shown in Figure 9 [22]. Repair of such damage could have been done through the use of aluminum doublers or composite patches. Doublers would introduce a second layer of skin which would be riveted over the cracked area. Rivets introduce additional locations where local stresses increase and therefore increasing the probability of crack initiation. Composite patches may be used and bonded to the wing surface to redistribute stresses without the addition of rivets. Composite patches were chosen as opposed to aluminum doublers and were applied to the skin as shown in Figure 10. The application of patches redistributed the local stress and therefore increased the life of the component. The addition of patches to the cracked areas limited further crack growth; this is demonstrated through the component life cycle graph found within Figure 11 . The two curves within the figure show an unpatched component and a patched component. Both components were loaded using identical conditions and only the unpatched component exhibited crack propagation. Similar repairs were carried out on cracked ribs of the Hercules aircraft within the RAAF fleet. 


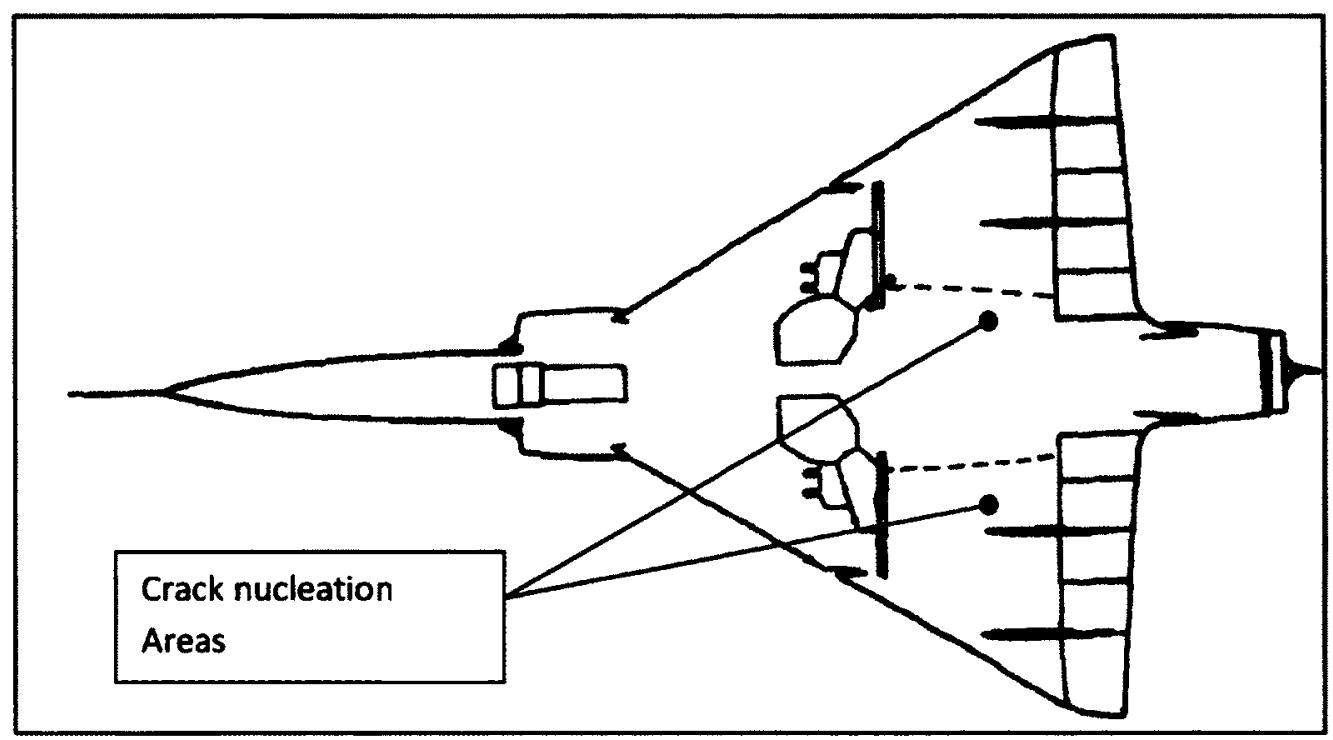

Figure 9: Australian Mirage III Crack Nucleation Locations [23]

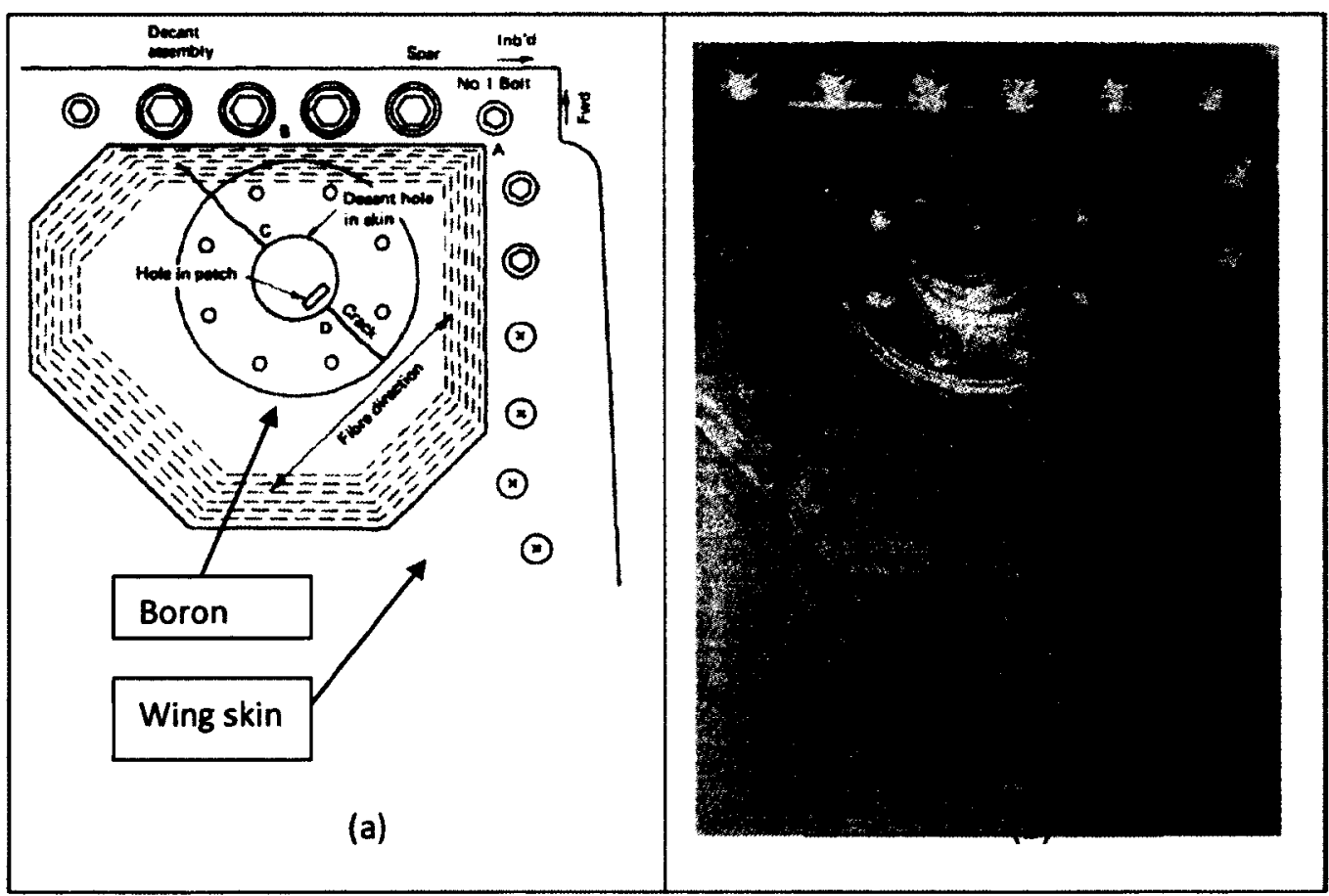

Figure 10: Australian Mirage III Patched Crack: (a) Repair Schematic and (b) Actual Repaired Wing Skin [23] 


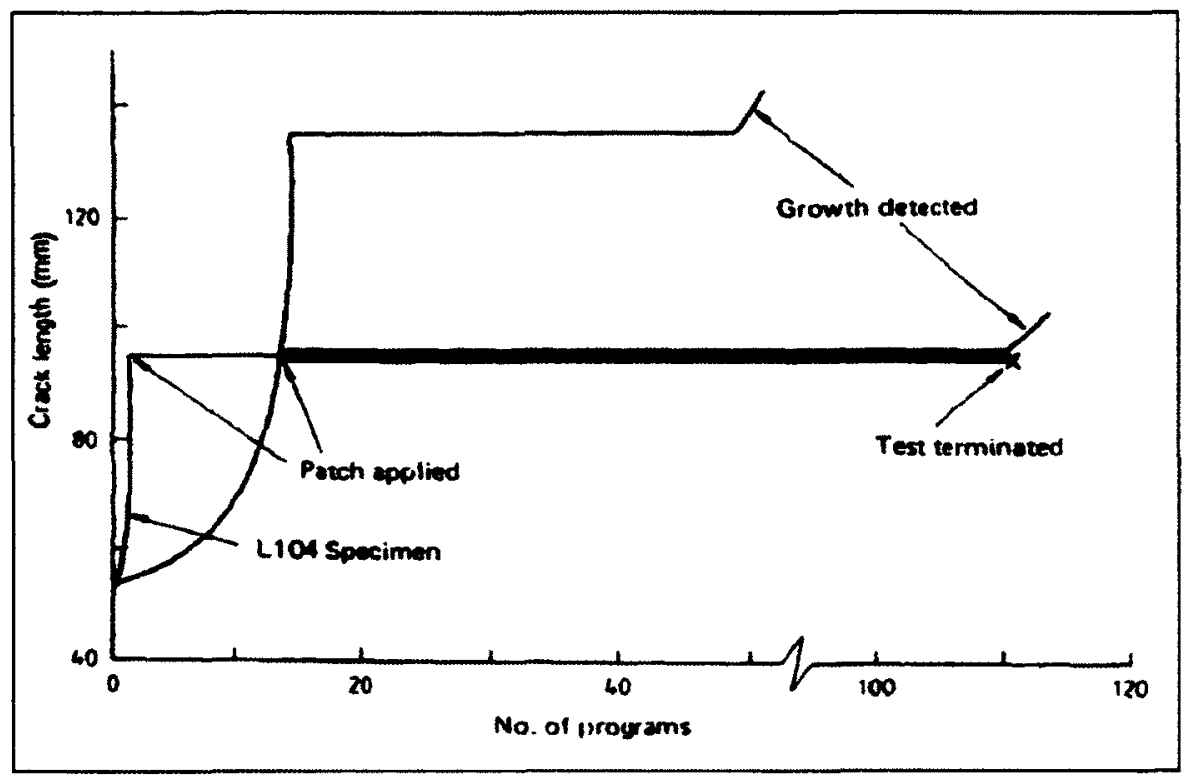

Figure 11: Influence of Patches on Crack Growth [23]

Consistency of all patch bonded repairs was achieved by following a well-accepted bonding process. The Boeing Corporation process for surface preparation consisted of a phosphoric acid gel-anodising procedure. There are two versions of this process; the first version is used on a production line for which components can be dipped in a chemical bath. The second version of this method was developed for in-field repair for which the component could not be submerged in a chemical bath [21]. The second variation is called the Phosphoric Acid Non-Tank Anodizing (PANTA) technique and was implemented on the mirage wing by the RAAF [22]. This process ensures that the surface is contaminate-free and is ready for bonding.

The use of composite patches along with a proven bonding process has shown to be an economical method for extending the life of aging aircraft. This method became the choice for repair as patches were easy to fabricate and were corrosive resistant $[\mathbf{2 4 , 2 5 ] . ~ H o w e v e r , ~ e v e n ~}$ with the best fabrication and installation methods, patch bonded repairs must still be inspected to ensure bond strength integrity. In some cases, bonds may appear to be safe; however the bond may not have any load carrying capabilities. These types of bonds are known as kissing 
bonds [26]. Kissing bonds are dangerous and unsafe for operation. Bonding of two components together using a well-accepted method may result in a strong, weak or kissing bond. Kissing bonds appear to have full adhesion between the surfaces; however, the bond fails well below the design limit [26]. The failure may occur at $20 \%$ of the design load [21]. For that reason and to avoid premature failure, it is important to investigate the integrtity of any bond, whether it be between metal-to-metal, composite-to-metal or composite-to-composite.

Inspection of patches using advanced technology is necessary to ensure safety [27]. Inspection of these patches may be done using the aid of advanced scanning techniques. Advanced techniques include Non-Destructive Evaluations (NDE) and Structural Health Monitoring (SHM) Systems $[27,26]$. NDE techniques may include technologies such as ultrasound inspections using A-Scans, B-Scans, or C-scans (later described in section 2.1.1) [27]. SHM techniques include a variety of new experimental technologies such as Acousto-Ultrasonic (AU) and Capacitance Disbond Detection Technique (CDDT) $[28,29]$.

All patches as well as their inspection technique must be certified so that an aircraft may maintain airworthiness. Possible inspection techniques are discussed within the following section.

\section{I.2 Inspection of Structures}

Safety is of utmost importance for all types of air transportation, especially for military and civilian uses. Newly manufactured aircraft structures are scrutinized by quality control and repaired structures are inspected within the field to prolong the life of the component. Determination of how long a component is to properly function within in an aircraft can be achieved in many ways, one such way is through the use of a Holistic Structural Integrity 
Programs (HOLSIP) as outlined by the National Research Council Canada (NRC). HOLSIP is a physics-based model where specific data regarding usage, damage and material properties may all be used to determine the remaining life of the component as shown in Figure 12 . Inspection of damage within components as part of this approach is primarily carried out using NonDestructive Evaluation (NDE). NDE is a process in which specialty technology is used to interrogate the health of a structure for flaw or damage detection. Structural Health Monitoring (SHM) as defined by Airbus is as follows: "The basic approach is to make non-destructive testing technology to become an integral part of the aircraft structure itself" [30]. This integration is done for the detection of defect formation during routine maintenance or in-service operation $[30,31]$. The following subsections describe some NDE and SHM available technologies.

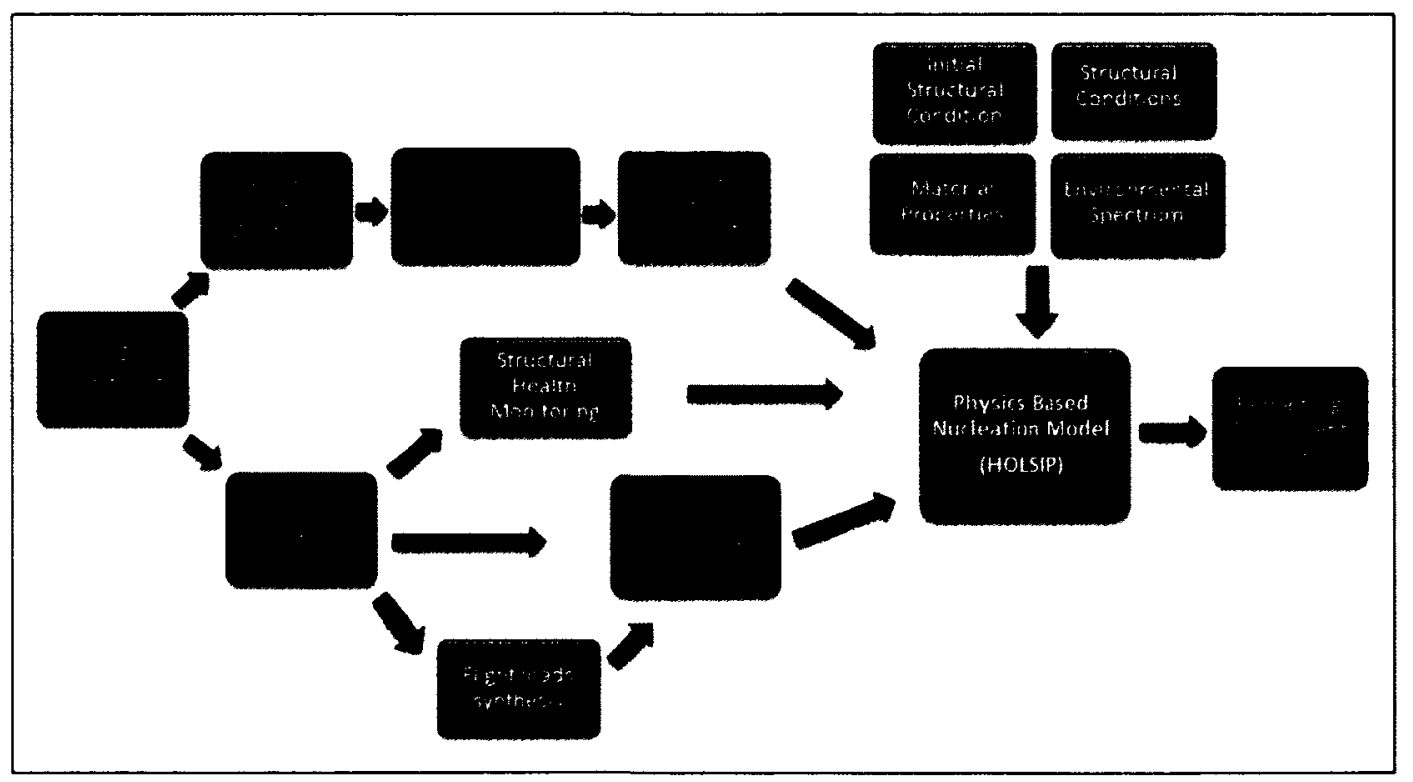

Figure 12: National Research Council Canada Holistic Structural Integrity Program

\subsubsection{Non-Destructive Evaluations (NDE)}

Non-Destructive Evaluations (NDE) were first developed for aircraft in the 1960 s and simulations along with analytical models followed shortly within the 1970s. Recent developments in sensors and signal processing have also played an important role in the growth of NDE [32]. 
There are several NDE technologies that are used and trusted within the aerospace industry; however, each has their advantages and disadvantages for the detection of different types of defects. The seven NDE techniques used within industry are listed in order of popularity: visual inspection, ultrasonic, eddy current, acoustic emission, X-Ray radiography, thermography and shearography.

\subsubsection{Visual Inspection}

Visual inspection is the most common form of NDE used today for in-service aircraft. This technique involves having inspectors closely examine components using their eye sight for surface or near surface defects. Due to the limitation of the human eye, technicians utilize additional tools such as static optical microscopes or even scanning electron microscopes to view damage. Visual inspection is limited by the viewing capabilities of the human eye. Therefore, it may be difficult to identify the severity of damage, especially for micro-cracks. In many cases where visual inspection reveals damage, components are removed from the aircraft and sent out for further inspection utilizing sophisticated technologies.

In addition, new illumination chemicals (known as dye penetrants) have been utilized on cracked surfaces to detect cracks under special lighting [32]. These chemicals are applied to the surface of a structure and small amounts of the chemical seeps into cracks while the remainder of the chemical is removed. Under special lighting, this chemical illuminates and reveals the crack. This technique is very limited to optical viewing capabilities and is therefore very difficult to implement on composite structures where damage occurs under the surface as delamination or micro cracking within the matrix or fibres. 


\subsubsection{Ultrasound}

Ultrasound (UT) inspection (also known as ultrasonic inspection) is an NDE technique in which sound waves are guided through the structure for detection and evaluation of damage. Through the analysis of UT signal attenuation, phase shift, reflection and scatter, damage can be found and quantified. There are two forms of signal propagation methods that are used for UT: pulseecho and pitch-catch. Pulse-echo is a method in which a single transducer is used to send the UT signal and the same transducer acts as a receiver which collects all the signals after they have been reflected by edges and defects. Pitch-Catch is a method in which two transducers are used. One transducer transmits signals while the other receives the signals. Both pulse-echo and pitch-catch methodologies are used in different scan types to provide different image resolutions.

There are three forms of ultrasound scan types: A, B and C scans; each one of these scans is used for a specific application and provides different image resolution of damage. A-Scans are the most basic form of UT inspections, they are used to scan as single point for damage; this is done by scanning through the thickness of the component. Signals are sent from one free surface and allowed to bounce back off another free surface so that it may be captured by the transducer as shown in Figure 13. In the presence of damage, signals will reflect off the damage and will be received by the transducer prior to receiving the backwall echo. Signal propagation using pulse-echo of a component with a defect is shown in Figure 14. 


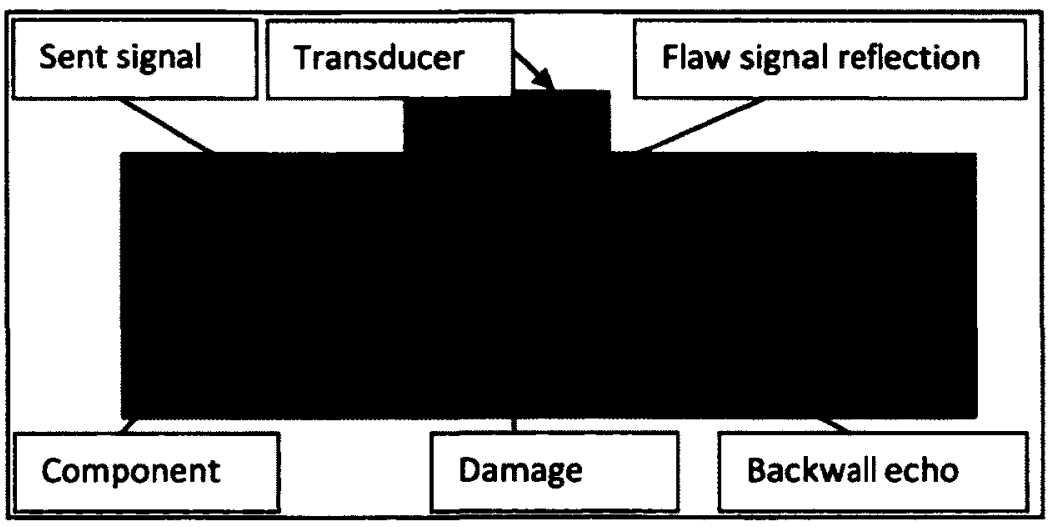

Figure 13: Point Analysis using A-Scan

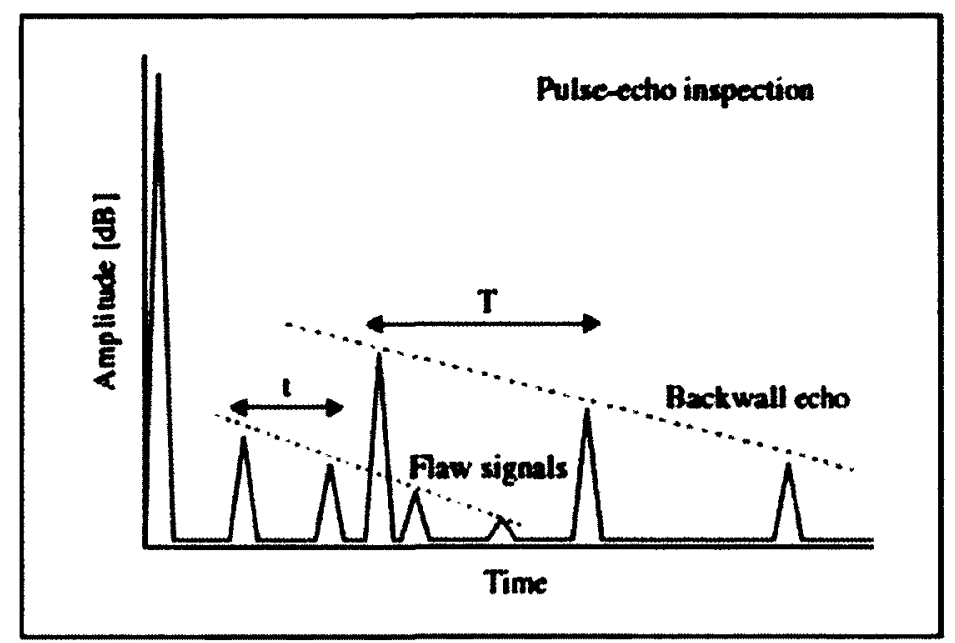

Figure 14: Ultrasonic Signal of Pulse-Echo [32].

B-Scans are a series of A-scans which are carried out along a continuous line. By scanning along a line, a B-Scan provides results for cross-section analysis; this is utilized to determine the depth of any damage. C-Scans are a grouping of B-Scans that are compiled into a single image. This type of scan is used to scan large surface areas. C-scans are used to identify the location damage anywhere below the scanning surface.

UT inspections using either A, B or C-scans are capable of identifying a variety of damages found within composites such as: delamination, fibre waving, voids, fibre cracking, and matrix cracking [33]. Its ability to detect damage makes it ideal for any damage types; however, the largest drawback to UT is initial equipment cost. More on UT technology is found in section 2.1.1. 


\subsubsection{Eddy Current}

Eddy current is an NDE technique in which a probe is used to measure the change in electromagnetic impedance of a conductive structure. Change in the impedance is caused by a change in strain within the structure which is usually caused by a defect [32].

The probe used for this NDE consists of two coils, one which is subject to an alternating current, while the other is only subject to the produced magnetic field. Eddy currents are developed within the conductive material in the presence of the electromagnetic field. The eddy current consequently produces a counter acting field which is picked up by the secondary coil. Changes within the received magnetic field are a result of defects which hinder eddy current flow. Setup using a two-coil probe is shown in Figure 15 below.

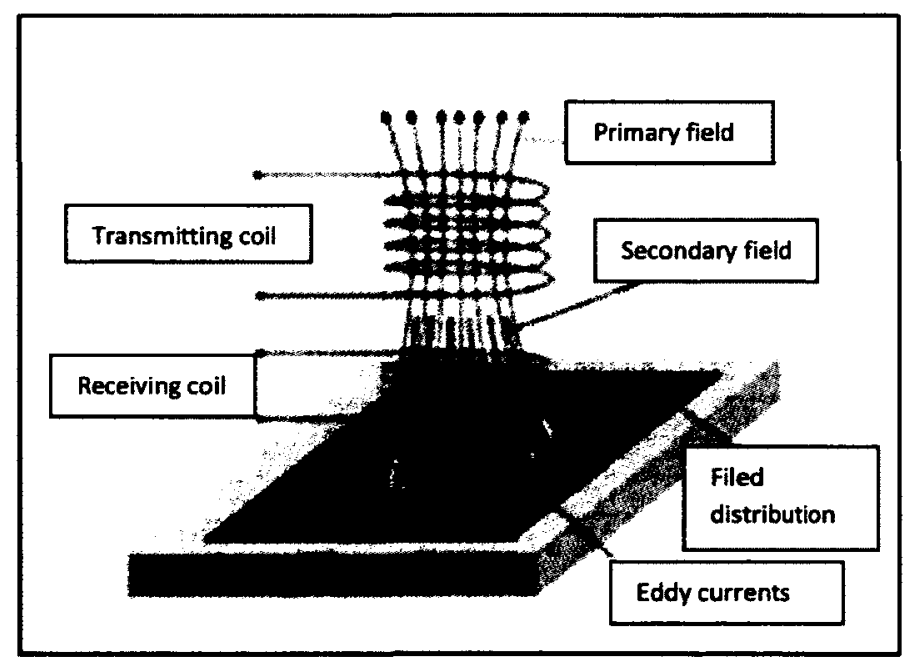

Figure 15: Eddy Current NDE [30]

Eddy current is suitable for the detection of cracks around rivet holes and strains within thin structures. This method is well established for metallic materials but in recent years this technology has been under evaluation for use on composite materials. This technique is favourable when compared to UT as the equipment used to carry out testing is smaller and less expensive. 


\subsubsection{Acoustic Emission}

Acoustic Emission (AE) is a passive NDE technique in which there are no transducers, just sensors. Materials have stored elastic and plastic energy that are a result of applied loading. Rapid release of stored energy results in acoustic waves which propagate through the structure. Release of energy is a result of crack nucleation, crack propagation, fracture, fibre breakage, matrix cracking or slippage of grains within the metallic structure. AE waves are registered by sensors which are placed along the structure. Features such as signal duration, amplitude, energy and travel time are used to determine different types of defects. Signals generated by the structure have frequencies that can range anywhere from $10 \mathrm{KHz}$ to $1 \mathrm{MHz}$ [32].

Sensors used within the AE system are made of piezoelectric elements. These sensors are bonded on the structure to form a large grid. Waves generated through rapid release of energy register in many of the bonded sensors. Through triangulation using flight time of the signal, algorithms are able to identify the location of the damage. However, signal processing is very difficult as there is usually a lot or significant background noise.

\subsubsection{X-Ray Radiography}

X-Ray Radiography is an NDE technique which utilizes X-Rays or Gamma rays to view defects within the structure. Figure 16 shows an illustration of Radiography; this technology involves the transmission of radiation onto the structure. A portion of the radiation is absorbed by the structure in areas of defects. The unabsorbed portion of radiation which passes through the structure is captured onto a photographic film. Defects within the structure which have absorbed radiation appear on the film as areas with higher absorption [32]. 


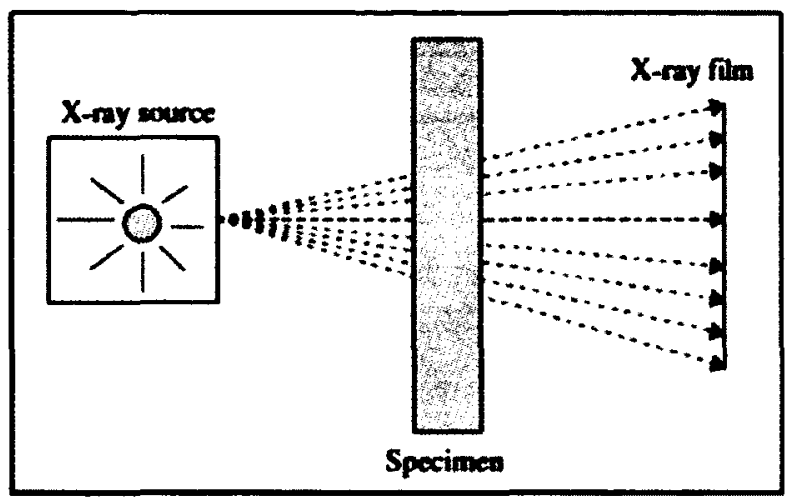

Figure 16: Radiography [32]

\subsubsection{Thermography}

Thermography is an NDE technique in which thermal conductive properties of a structure are captured using an infrared camera to detect damage within a structure. Figure 17 illustrates the use of thermography as an NDE technique. In this NDE technique, the structure is exposed to a thermal source which may be induced by a flash lamp or lasers. Propagation of heat caused by either the flash or laser through the structure is done using an infrared camera as illustrated in Figure 17. This process is done prior to damage formation to form a benchmark image result. After the formation of damage, the same heat pulse is allowed to propagate through the structure and is captured by the same camera. Damaged locations will have changed local thermal conduction and absorption properties. Subtraction of the latest images from the benchmark image will reveal locations for which transmission of heat has changed; these areas translate into defect locations $[32,34]$. A more in-depth description of thermography can be found in section 2.1.2. 


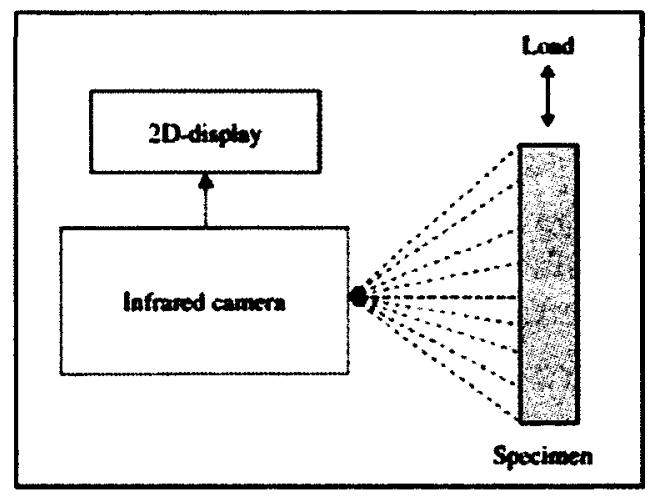

Figure 17: Thermography [32]

\subsubsection{Structural Health Monitoring (SHM)}

Health Monitoring (HM) is the analysis of a systems performance, throughout its life cycle; it is applicable to aerospace structures, engines, civil structures and many other systems. Each application of $\mathrm{HM}$ is done in a different manner and for a different purpose. For example, HM on engines is done to collect and analyze specific parameters on the performance of an engine to maintain highest running efficiency and to extend the life of an engine. While HM on structures are done to detected damage formation prior to component failure so that repair is cost effective and safety is not compromised. When HM is applied to aerospace or civil structures, it is known as Structural Health Monitoring (SHM).

SHM is very similar to NDE as it is used in the detection of damage for new and in-service aircraft. SHM integration within a structure is very similar to a human nervous system [30], as shown Figure 18. The system consists of two parts: the interrogator and the sensor network. The interrogator acts in similar manner as the brain while the sensor network acts as the nerves within the human tissue. While SHM systems may vary in design, complexity and purpose, they always have both an interrogator and a sensor network. 


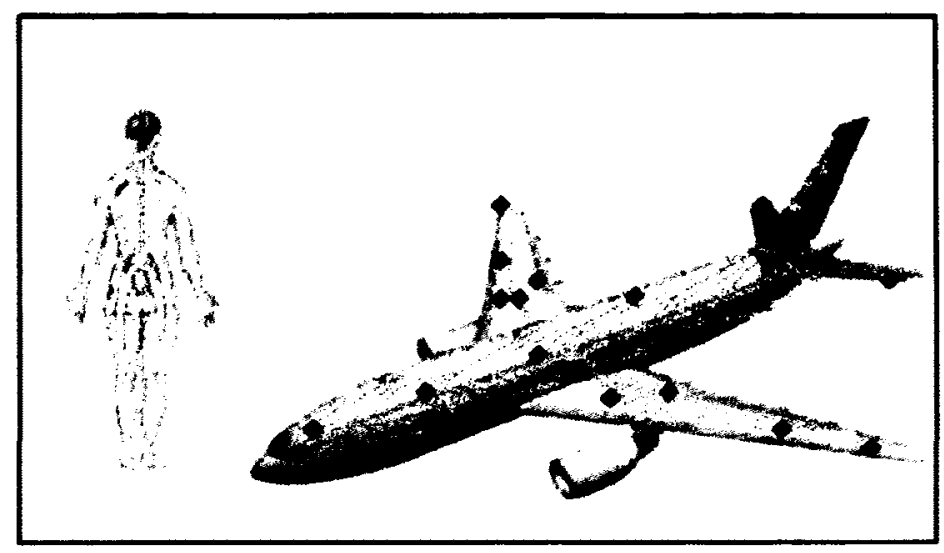

Figure 18: Aircraft Nervous System [30,31]

There are two basic approaches to SHM design; the first is focused on the reduction of maintenance cost while the second is focused around the design of new aircraft. Reduction of maintenance cost can be achieved by carrying out maintenance when indicated by the onboard SHM system. However, this will not be achieved until SHM systems are found to be reliable. Establishing system reliability will be achieved by first implementing SHM systems as data collectors. During routine maintenance, SHM monitored areas will be inspected and damage evaluated. Once these SHM systems have been found to be effective, maintenance will be carried out once indicated necessary by the SHM systems. The reduction in maintenance has a potential cost savings of up to 75\% [35]. In addition to the implementation of SHM systems for maintenance based analysis on older aircraft, SHM will be used to aid in the design of next generation aircraft. New aircraft will be under-designed to be lighter and more fuel efficient as there will be less need for redundancy of aircraft structure [35]. However, prior to integration on new or old aircraft, the SHM systems must be tested in the laboratory to evaluate their effectiveness and reliability for the detection of damage initiation and growth.

There are two major types of SHM systems available on the market: health monitoring and load monitoring [30]. Health Monitoring Systems (HMS) are those that directly seek defects within a structure, while Load Monitoring Systems (LMS) monitor flight parameters which in turn are 
used to predict probability of defects [30]. LMS are used to monitor loads and strains using Fibre Bragg Gratings (FBG) or strain gauges. HMS are used to detect structural damage through the use of: Eddy current, Capacitance Disbond Detection Technique (CDDT), Comparative Vacuum Monitoring (CVM), Surface Mountable Crack Detection System (SMCDS) and Acousto-Ultrasonic (AU). The following sub-sections describe each of the passive and active systems and their basic physics.

\subsubsection{Fibre Bragg Gratings (FBG)}

Stresses produced during loading of a structure may be found by knowing both the modulus of the material and the strain of the materials. Fibre Bragg Grating (FBG) is one technology that may be used to measure strains.

FBG technology relies on light physics to calculate the strain at given points along a component; this is achieved by passing light through a specialized fibre optics cable. A fibre optics cable is built of glass fibre with gratings at predefined intervals, as shown in Figure 19. Gratings are areas along a fibre which have different light reflection properties; these gratings are produced using laser etching during fibre manufacturing. During the application of load, the fibre elongates; this in turn causes the distance between gratings to change. Change in spacing results in a change in the refractive index of the fibre [32]. Due to the change in refractive index, specific wavelengths will be reflected back along the cable while the remainder will flow right through. Equation 1 shows the relationship between the reflected wavelength and grating spacing [32]. By knowing the reflected wavelength, the system uses Equation 1 to calculate the grating spacing which is proportional to that of the strain of the component [32]. 


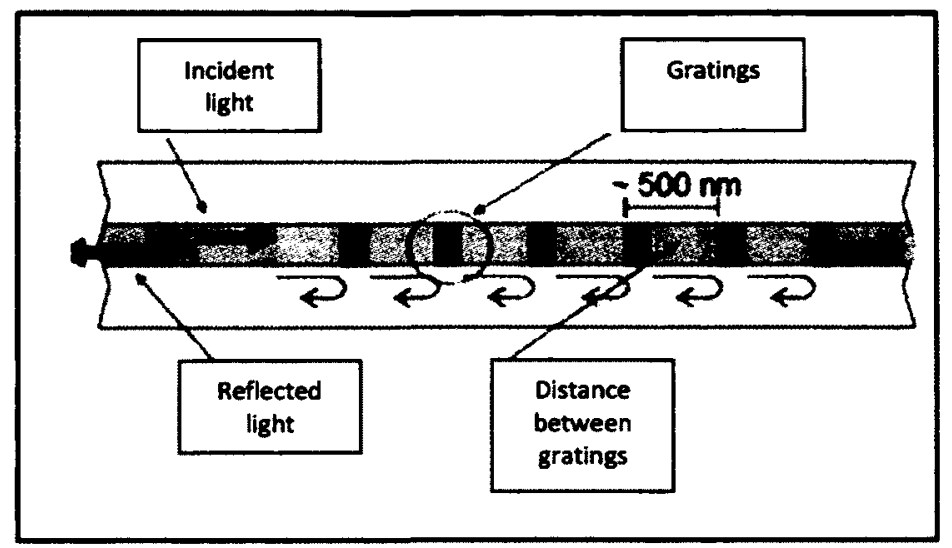

Figure 19: Fibre Bragg Gratings [36]

$$
\lambda_{w}=2 n \Lambda
$$

Equation 1

Where:

- $\lambda_{w}$ is reflected wave length,

- $\Lambda$ is the spacing between gratings and

- $n$ is the average refractive index.

FBG contain a large number of gratings along a single fibre; for that reason they may be used to measure strain in many different locations along a single fibre using the same acquisition system and in/out port.

FBG have been tested in the past on military aircraft such as the F-22 and significant research has been carried out on both metallic and composite components. One of the largest drawbacks to FBGs is integration with the structure. There are two integration methods, internal to the structure or external to the structure. Internal integration within composite materials allows the FBG to be embedded along with many fibres within the laminate. However, FBG fibres are significantly larger than common carbon and boron fibres which make the area around them susceptible to failure within the matrix. 


\subsubsection{Strain Gauges (SG)}

Strain gauges (SG) are another type of sensors used to monitor loads on a structure and are very similar to FBG. Unlike FBG, SG relies on electrical physics to calculate the strain at a particular location on the component. Strain measurements of the component are obtained by passing current though the sensor and measuring its resistance, which is a function of the strain experienced by the SG. A simple SG, such as the one shown in Figure 20(a), is composed of a series of wire windings. When elongated or compressed, the windings experience a change in cross-sectional area of the wire. The change in area limits or promotes the passing of electrons (i.e. changing the resistance of the sensor) $[37,38]$. The change in resistance can be calculated by placing the SG into a quarter-bridge strain gauge circuit as illustrated in Figure $20(b)[37,38]$. By knowing the resistance, one can calculate the strain within the area of the gauge.

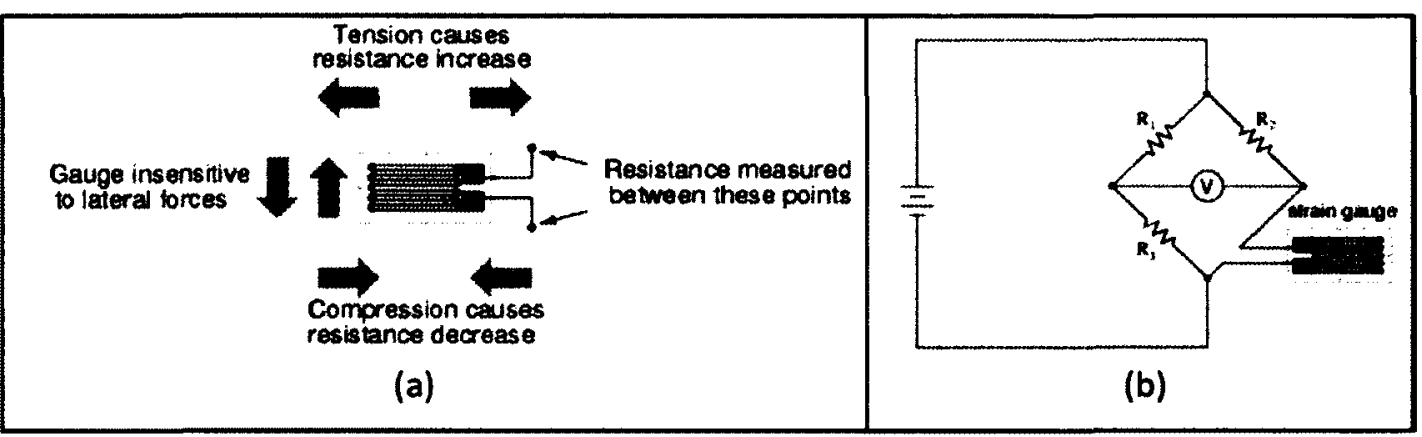

Figure 20: Strain Gauge and Circuit: (a) Typical Strain Gauge; (b) Typical Quarter Bridge Circuit [37]

Both the FBG and SG sensors output the same type of information (i.e. strain). Damage identification using either FBG or SG is done by comparing the output strains to a predetermined aircraft load strain model. Areas which output significantly higher or lower strains, as compared to the model, indicate the presence of damage [32].

\subsubsection{Eddy Current}

Eddy Current technology for SHM utilizes the same principles as those for the NDE technique described in section 1.2.1.3 [30]. However, unlike NDE sensors which are handheld, SHM 
sensors are more compact and are surface mountable, see Figure 21. Eddy current sensors are predominantly used for detecting defects within metallic structures; however, it has been shown that they can also be used on conductive composite materials [29].

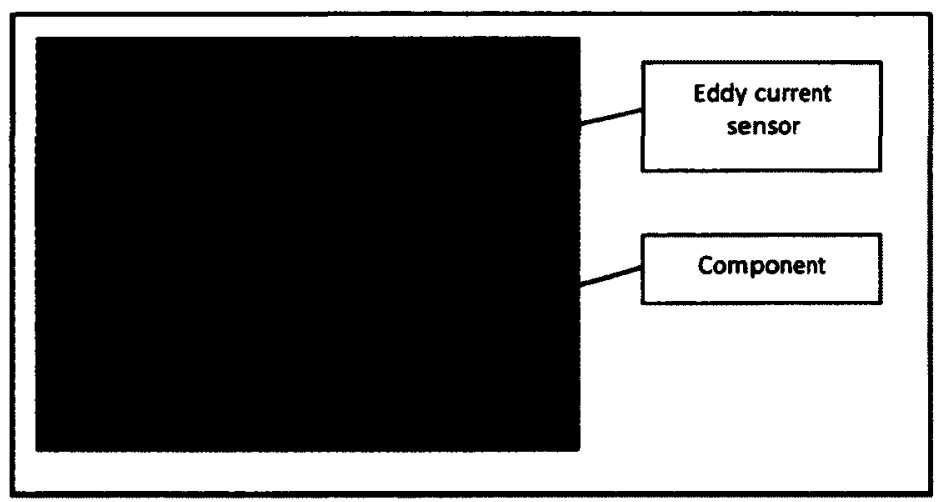

Figure 21: Typical Eddy Current Sensor [30]

\subsubsection{Capacitance Disbond Detection Technique (CDDT)}

Capacitance Disbond Detection Technique (CDDT) is based on the measurement of changes in capacitance of adhesively bonded joints [29]. This method treats the component under inspection as a capacitor. Two components joined together resemble the end plates of a basic capacitor and the adhesive resembles the dielectric between the endplates as illustrated in Figure 22(a) and Figure 22(b). As the joint disbonds, the distance between the two composite end plates increases (Figure 22 (b)) and therefore the capacitance drops; indicating disbonds [29]. To utilize this technique, an initial reference capacitance must be acquired for each component; changes in this capacitance indicate a change to the bond integrity [29]. This technology has been tested at the National Research Council Canada (NRC) and the basic capacitor equation was transformed to meet their specific application to Equation 2 [39]. More on this technology is found in section 2.2 . 


$$
\% \text { Disbond }=\frac{\frac{c}{K_{\text {Adhesive }} \varepsilon_{0} A}-\frac{1}{d_{\text {bond }}}}{\frac{1}{d_{\text {disbond }}}-\frac{1}{d_{\text {bond }}}}
$$

Where

- $\quad c$ is the change in capacitance;

- $d_{\text {disbond }}$ is the disbond thickness in inches;

- $K_{\text {Adhesive }}$ is the adhesive dielectric constant;

- $d_{\text {bond }}$ is the bond thickness in inches;

- $\varepsilon_{0}$ is the permittivity of free space and is equal to $8.854 \times 10^{-12} \mathrm{~F} / \mathrm{m}$; and

- $A$ is the bond area

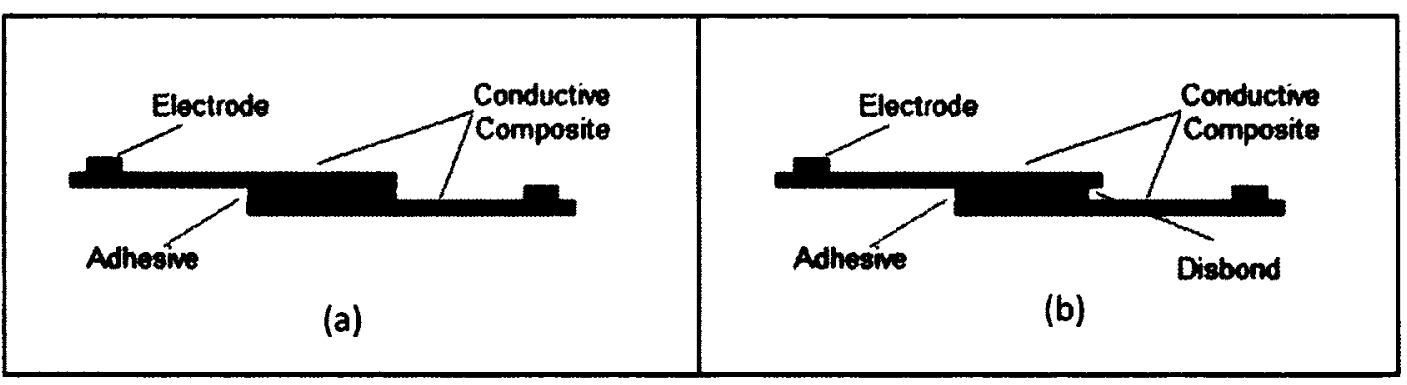

Figure 22: Capacitance Disbond Detection Technique: (a) Bonded Component; (b) Disbonded Component

\subsubsection{Comparative Vacuum Monitoring (CVM)}

Comparative Vacuum Monitoring (CVM) is a technology in which a series of capillary tubes are setup along the surface of the structure as shown in Figure 23. Each alternating capillary is open to atmospheric pressure while all remainder capillaries are subject to a vacuum pressure as illustrated in Figure 23. As a crack propagates across the capillaries, it connects the capillaries and therefore a pneumatic leak occurs. The sensor then detects a leak and therefore identifies the presence and location of a crack. Also, as the crack grows, it crosses multiple capillaries; by knowing the distance (d) between the capillaries, the length of the crack can be estimated [40]. 
CVM sensors can only be mounted on the surface of a structure and therefore can only detect surface defects, primarily cracks. This technology also requires continuous vacuum pressure so an air compressor must be running continually while interrogating the structure.

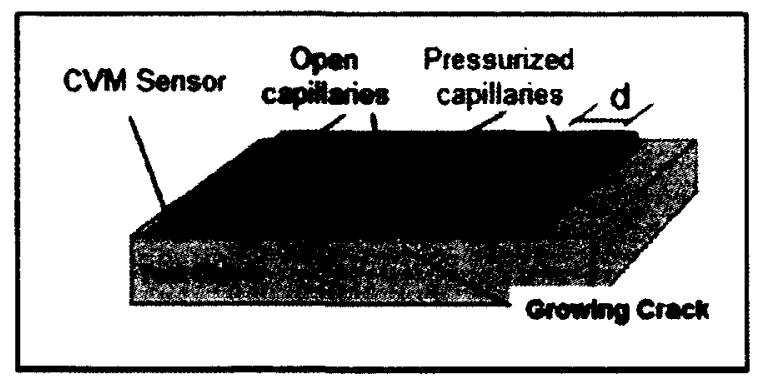

Figure 23: CVM Sensor [30]

\subsubsection{Surface Mountable Crack Detection System (SMCDS)}

Surface Mountable Crack Sensors (SMCS) are very similar to that of CVM sensors. However, SMCS are composed of a conductive paint rather than capillary tubes as illustrated in Figure 24 . When the component is being interrogated, low current with will pass through the wire. When cracks grow through the SMCS, they break the paint. The conductive path changes to an open circuit and the system identifies the presence of a crack. The location of the crack is determined by the location of the broken conductive paths. Similar to CVM, the size of the crack can be determined by knowing how many paint strips the crack has gone through [41]. More information on SMCS is found in section 2.2.2.

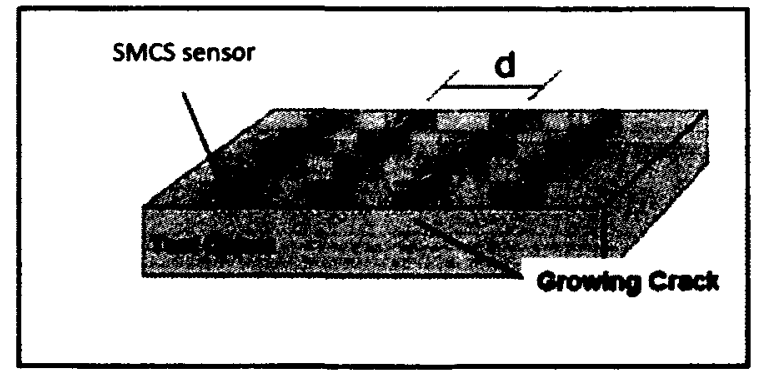

Figure 24: SMCS Sensor 


\subsubsection{Acousto-Ultrasonic (AU)}

There are two types of Acoustic methods that are being used in SHM: Acoustic Emission (AE) and Acousto-Ultrasonic (AU). Both methods rely on a component's ability to carry elastic waves. Both the AE and AU systems are composed of the same sub systems: piezoelectric ceramic sensors (Figure 25(a) \& Figure 25(b)) and the data acquisition system (Figure 25(c)) [32]. For AU, the sensors act as both a transmitter and a receiver [32]. In this technology, a benchmark reading is taken prior to any loading of the components. A benchmark reading is a collection of signals which are captured prior to any damage formation and they define wave propagation of a healthy structure. At later stages within the life of the component, signals are transmitted and received. Ideally, the received signals will be similar to the benchmark signals. However, due to the presence of damage, signals will be subject to attenuation, scattering and phase shifting, all of which indicate damage [32]. More on AU can be found in section 2.2.3.

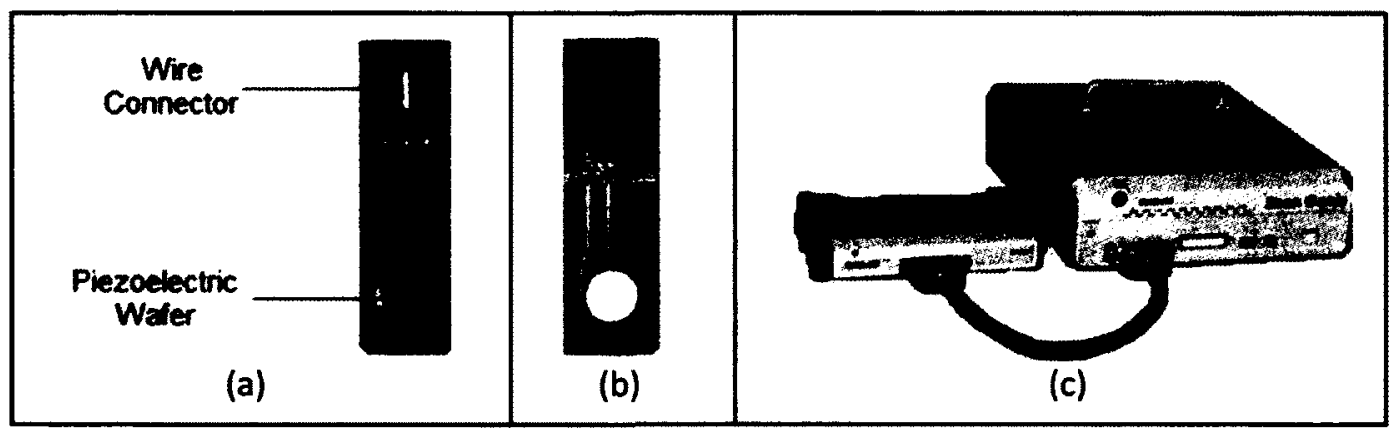

Figure 25: Acousto-Ultrasonic: (a) Sensor, Upper Side; (b) Sensor, Lower Side (Bond Surface); (c) Data Acquisition [42]

\subsubsection{Acoustic Emission (AE)}

Acoustic Emission (AE) SHM is similar to that of the AE used as an NDE technique described in section 1.2.1.4. This technology does not use any wave transmission devices; rather it listens for waves as they are generated by external sources or release of stored elastic energy $[32,43]$. 


\section{Inspection of Patch Repairs}

The introduction of patch bonded repairs especially by the Royal Australian Air force (RAAF) has led to an increase in research for analysis techniques required for certifying these repairs. The certification process has led to numerical model developments as well as NDE and SHM system evaluations for early detection of patch failure.

Numerical models for the evaluation of patch strength as well as crack growth of the repaired

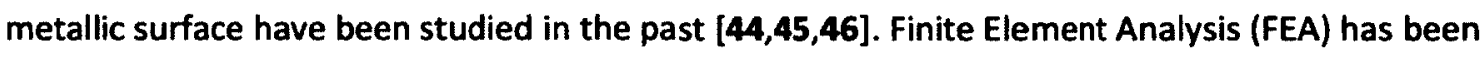
employed to analyze the effects of a patch on crack repaired aluminum alloys by Quinas [46]. Figure 26 shows an example of two models that were developed within FEA software to see the effects of a patch on the stress intensity factor $\left(K_{l}\right)$ by Quinas. These two models were tested and evaluated, results of which are presented in Figure 27. It is clear from Figure 27, that the use of a patch reduces the crack's stress intensity factor and therefore extends the life of the component. In addition to FEA, analytical equation development was also performed by ChorngFuh Liu and many others [47]. Equations were developed for the evaluation of the stress intensity factor; which was later used to predict the remaining life of the component. In 2007, Fujimoto looked at FEA for crack growth and life analysis for different crack and disbond fronts [44]. However, even with the best prediction software, it is necessary to inspect the patch repairs during service of the aircraft. 


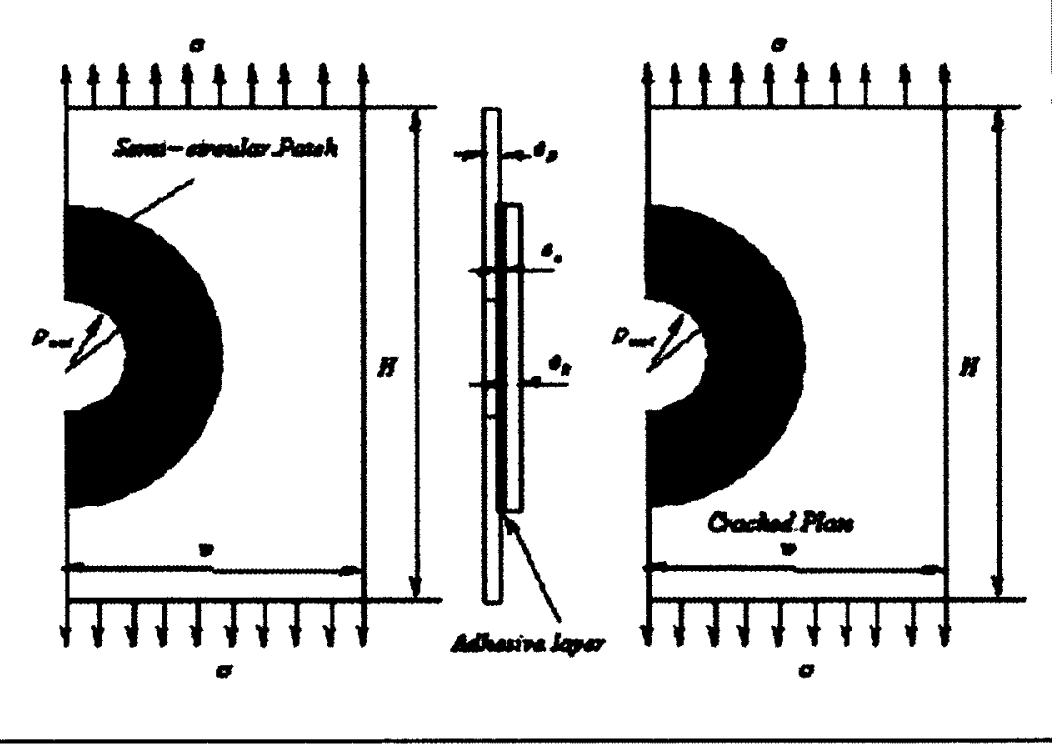

Figure 26: Simulated Patched Coupons: left displays a non-cracked coupon while the right is a cracked coupon [46]

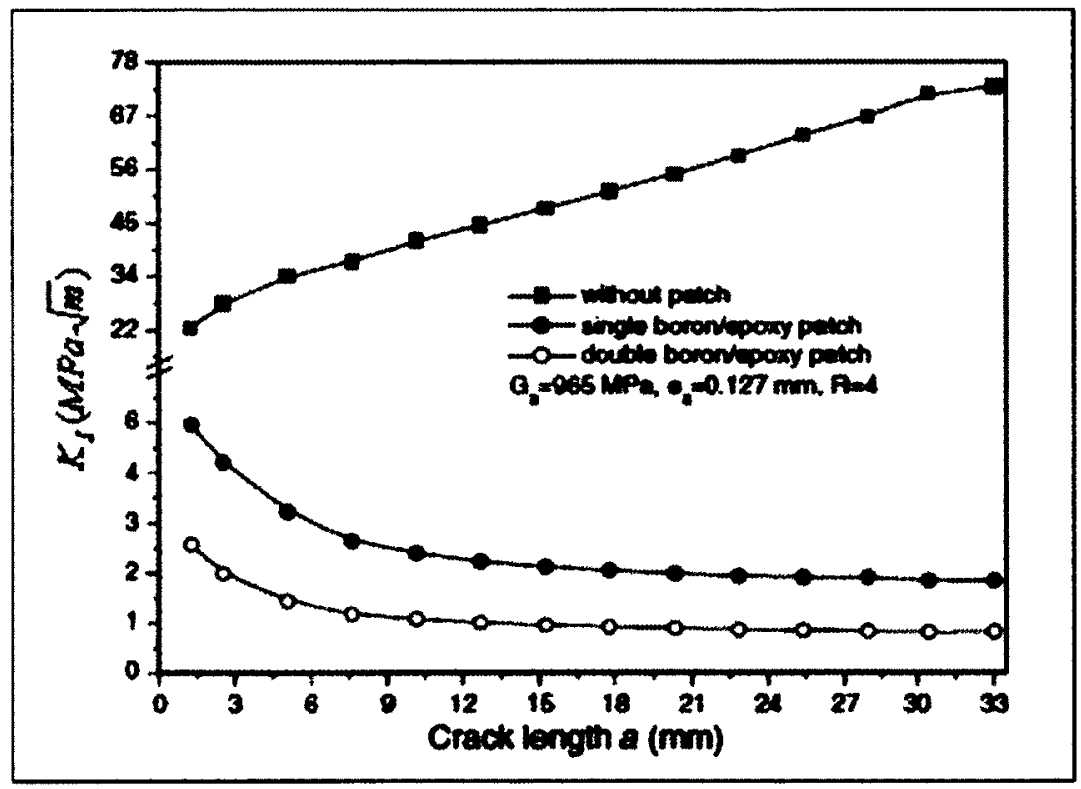

Figure 27: FEA Results for Patch Repaired Coupons [46]

NDE systems have been used to inspect patch bonded repairs. The inspection of patches could be carried out using Ultrasound, Thermography, Shearography, Non-linear ultrasonic, and Ultrasonic Resonance Spectroscopy $[26,29,39,48]$. All these methods have been tested on composite patches and have been proven to identify the location of and size of disbond. NDE 
techniques are also capable of detecting contaminants within the joint. Figure 28 shows the results of a patch scan using ultrasound; it is clear that this NDE technique is capable of identifying patch integrity. All of the above listed techniques are capable of identifying disbond; however, many of these fail to detect kissing bonds as there is full contact between the components and the adhesive. Of all these methods, only Resonance Spectroscopy demonstrated good results for the detection of kissing bonds [26]. All of the above mentioned technologies are more than adequate for the inspection of disbond. However, inspection using these techniques requires the removal of the aircraft from service; in extreme cases, the component must be removed from the aircraft. For that reason, there exists the need to develop SHM technologies that may inspect the integrity of the patch during regular operation of the aircraft.

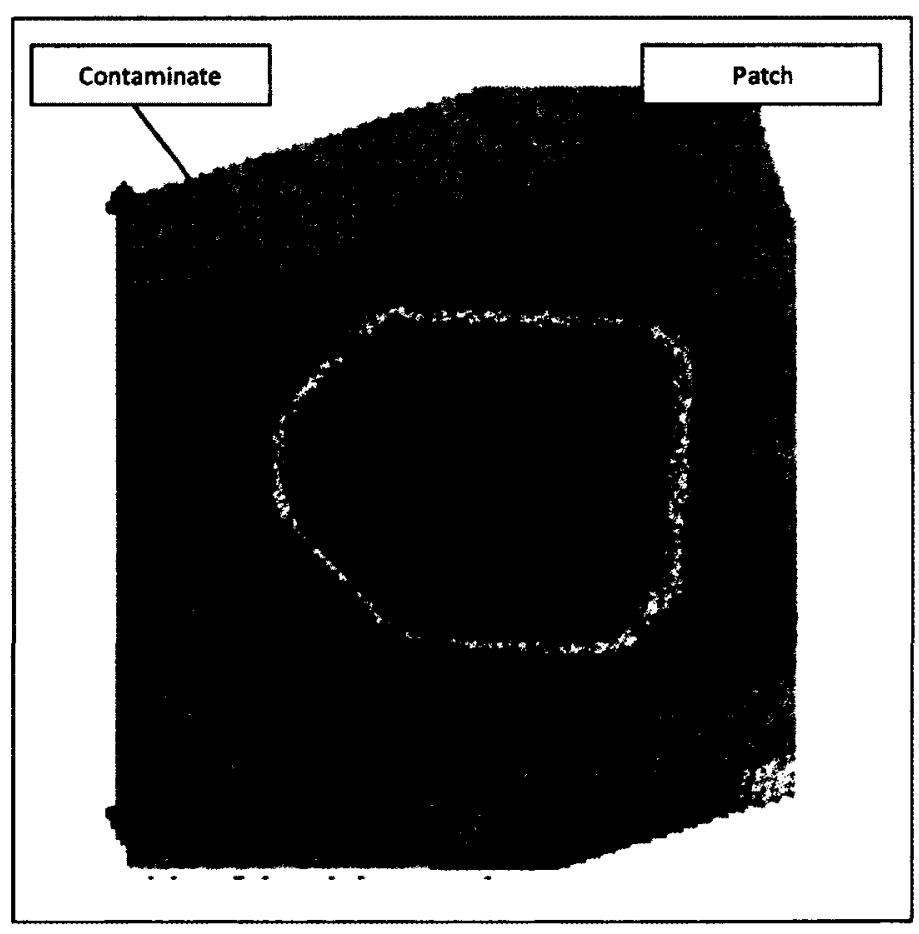

Figure 28: C-Scan of Patch [26]

Many SHM systems have been studied in recent years for their effectiveness of patch disbond detection [49]. Such SHM systems include: Acousto-Ultrasonic (AU) $[\mathbf{2 8 , 5 0 , 4 4 ]}$, Fibre Bragg 
Gratings (FBG) [25], Strain Gauges (SG) [27,51] and Surface Mountable Crack Detection System (SMCDS) [52]. All of these systems were tested on composite-to-metallic coupons and in many cases an attempt was made to monitor crack and disbond propagation. SG technology is one of the most basic forms of SHM technology used for monitoring patch disbond. Analysis of SGproduced data is easy and effective, provided that loads and strains on the component are well understood. SMCDS is also a very basic system which can be implemented to monitor crack formation within the adhesive along the edges of any patch. Amongst all described SHM technologies, $\mathrm{AU}$ has demonstrated much potential for the detection of different patch failure modes. However, this technology is susceptible to many external influences such as noise; thus making it difficult to analyze the data. There is much research that has been done using $A U$ systems and their underlying Lamb wave physics (later described in section 2.2.3). Extensive research has shown the effectiveness of $A U$ systems on isotropic mediums. However, there is ongoing research to test the effectiveness of this technology on composite structures.

All these NDE and SHM technologies have been tested and evaluated in past research. However, much of this research has been carried out on composite-to-metallic joints; none of the techniques have been evaluated on composite-to-composite joints. This thesis is focused on the evaluation of new SHM systems for their ability to identify and quantify disbond on compositeto-composite joints.

\subsection{Conclusion}

Composite materials have been replacing traditional metallic materials since the 1970 s. The amount of composites used in any particular industry is highly depended on their advantages and disadvantages for their specific application. While some industries have continued to increase the use of composites at high rates, the aerospace industry has progressed at a slower 
rate. The aerospace industry cannot tolerate failure and therefore a method of identifying the component's reaming life is of utmost necessity. NDE techniques have been used in the past to evaluate structural health; however, this process is time consuming and costly. The introduction of SHM technologies on aircraft may reduce inspection times as well as cost. 


\section{Chapter 2.0: Selection of Damage Detection Systems}

The focus of this thesis was to determine the effectiveness of multiple off-the-shelf Structural Health Monitoring (SHM) Systems for the detection of disbond of a composite-to-composite joint. There were many SHM systems mentioned within Chapter 1; however, only a limited number of them were used within this research study. The selected SHM systems were those which had the potential for the detection of defects in both composite and metallic structures. SHM systems were evaluated on metallic structures prior to composites, as metallic structures provide a less complex analysis scenario.

In addition to SHM systems, a number of Non-Destructive Evaluations (NDE) techniques were used to validate the performance of the SHM systems used within this study.

This chapter discusses all the SHM systems and NDE techniques used within this study as well as reason(s) for their selection.

\section{$2.1 \quad$ Non-Destructive Evaluation (NDE) Systems}

Inspection of coupons in this study was done using ultrasound as well as pulse thermography. Pulse thermography is not a certified NDE technique. However, thermography has been used in the past and has been shown to provide acceptable inspection results as those which were obtained using ultrasound for patch bonded repairs [29]. The following subsections describe both ultrasound and thermography inspections.

\subsubsection{Ultrasound Inspection}

Ultrasound inspection (UT) is one of the most utilized NDE techniques within the aerospace industry. UT, as mentioned in section 1.2.1.2, uses high frequency sound waves to interrogate 
the health of a structure. Waves are transmitted within the component using one of two methods, pulse-echo or pitch-catch $[32,53]$. The Pulse-echo method is a process in which signals are transmitted from a transducer which is located in close proximity to a receiver(s); or in some cases the transducer may act as the receiver. Induced waves are captured by the receiver after reflection off defects and other free surfaces as illustrated in Figure 29(a). Figure 29(b) shows an inspection signal using pulse-echo. Flaws within a structure will cause signal reflection back towards the receiver and transducer; this is known as the flaw signal as indicated within Figure 29(a) and Figure 29(b). The remainder of the signal (non-reflected portion) proceeds until reflection off a free surface of the component; this is known as backwall echo, as shown in Figure 29(a) and Figure 29(b). Flaw signals arrive prior to the backwall echo. The difference in time of flight (ToF) between the flaw signal and the backwall echo identifies the location of the defect and its distance away from the scanning surface $[32,53]$

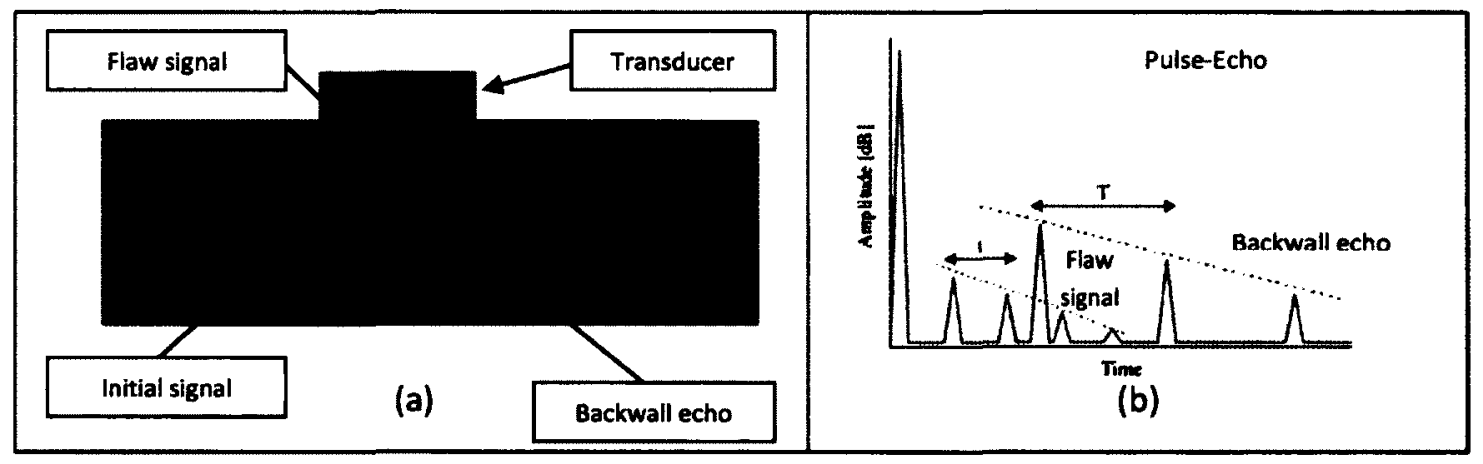

Figure 29: Ultrasonic Inspection using Pulse-Echo: (a) Component with Flaw and (b) Signal Results

Pulse-echo is very useful when access to a component is limited or the user is only interested in features which are located on one side of the component. If access is available for both sides, pitch-catch method may be implemented. Both the transmitter and receiver are separated by the thickness of the component; the transmitter is located on one side of the component while the receiver is mounted on the opposing side. Signals are transmitted through the structure and allowed to attenuate and disperse as they pass through structure. Ultrasound inspections are all 
performed using pulse-echo or pitch catch; however, displaying results of the scans can be done in one of three ways: A-Scans, B-Scans or C-Scans $[32,53]$.

A-Scans are the most basic form of analysis; the scan provides information related to a single point within the structure. The results of an A-Scan may be displayed on an oscilloscope or computer screen; displaying signal information as they propagate, attenuate and reflect off nearby surfaces as shown in Figure 30. The user of A-scans interprets signal reflection as damage within the structure [53].

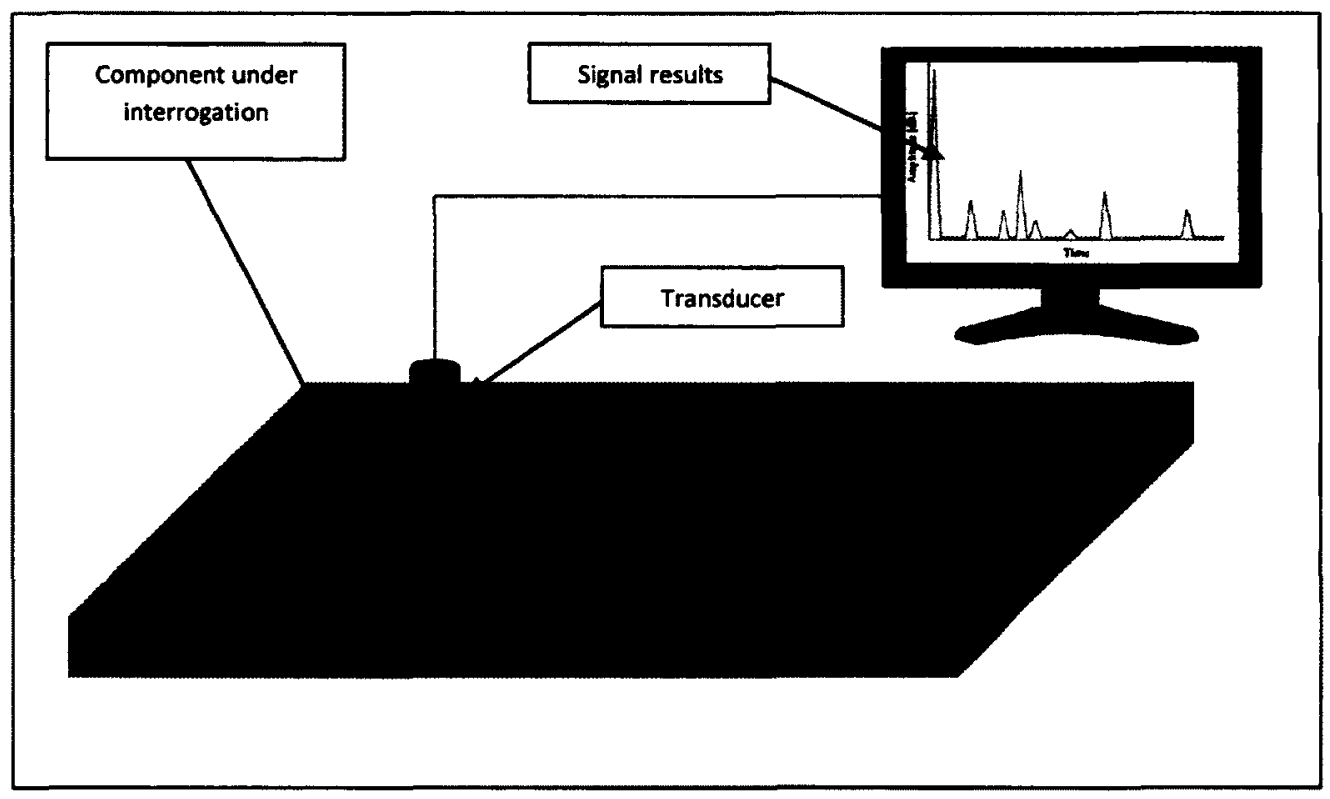

Figure 30: A-Scan of a Component with Damage

B-Scans are scans which are used to scan the cross-section of a component for the presence of defects. Through the inspection of multiple points along a continuous line, an image of the cross-sectional area may be constructed as shown in Figure 31 . The motion of the probe as illustrated in the figure accumulates multiple A-scans which are summed up to form the scanning image. Areas with no signal dispersion or partial reflection will appear as defect free while others will indicate defects as shown in Figure 31. This method is used to determine the depth of damage away from the scanning surface [53]. 


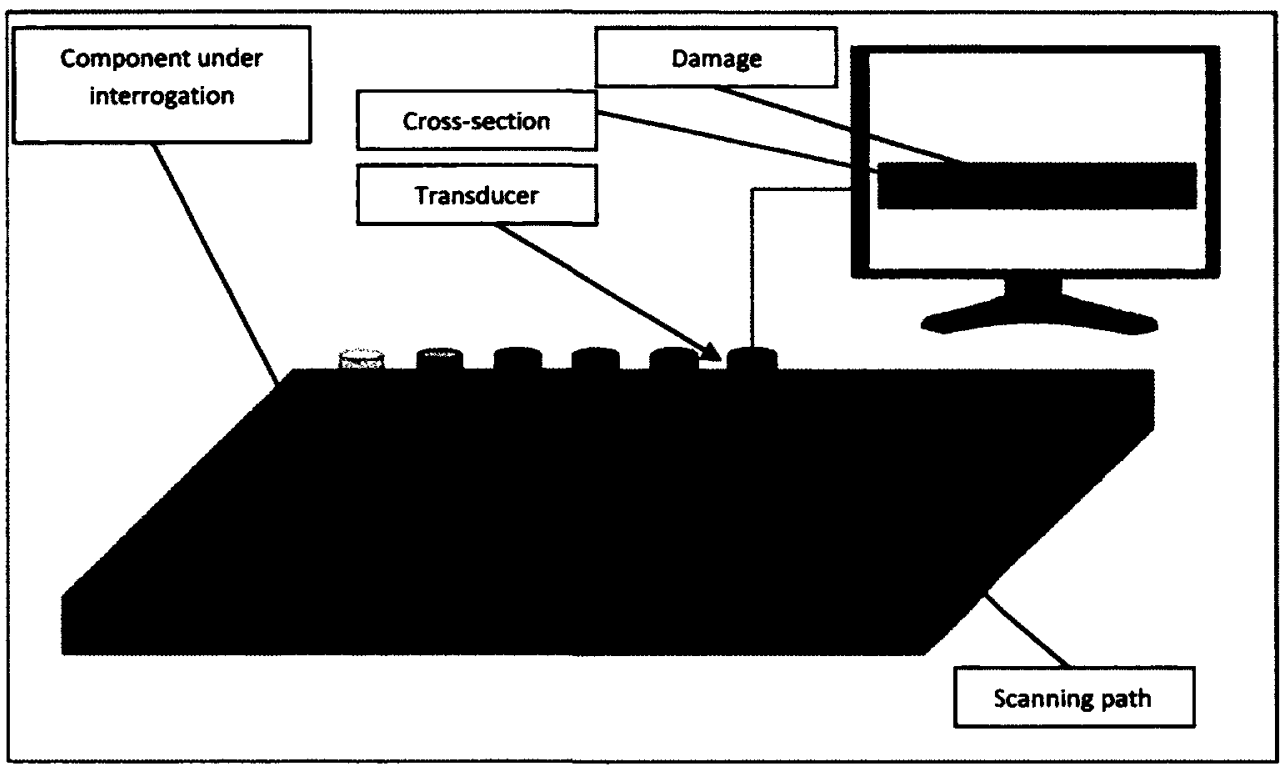

Figure 31: B-Scan of a Component with Damage

C-Scans are scans which allow for the inspection of an entire component. The inspection is carried out by scanning multiple points along a continuous line on the surface of the component as illustrated in Figure 32. Similar to B-Scans, C-Scans are a summation of scans along multiple lines which are along the scanning surface [53]. This inspection reveals damage which may be located anywhere beneath the scanned surface. Ultimately, A-scans as well as B-scans are carried out as part of the C-scan inspection as signal transmission required to construct a C-scan image belongs to B-scans as well as A-scans. 


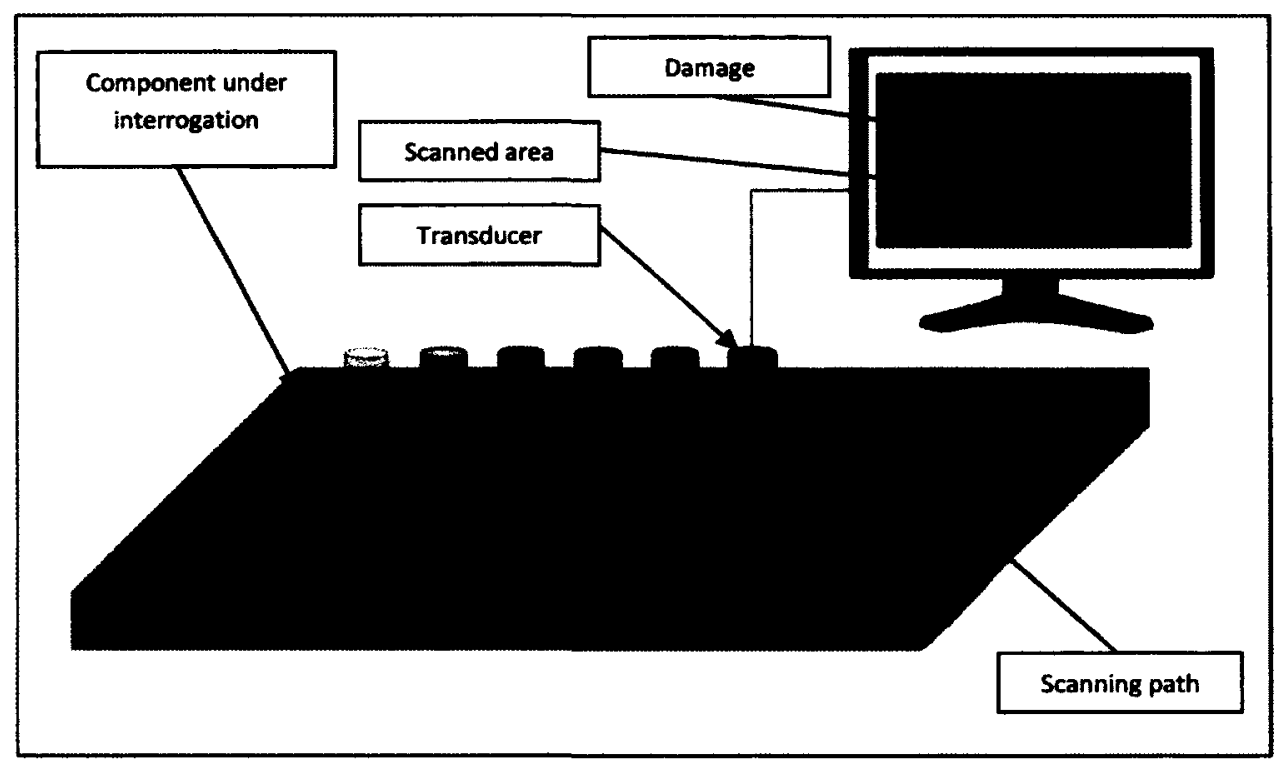

Figure 32: C-Scan of a Component with Damage

\subsubsection{Thermography}

Thermography as described in section 1.2.1.6 is a process in which the integrity of a structure is examined through the analysis of heat diffusion rates. Inspection of structures is done through the application of a thermal shock and the analysis of propagation of the thermal shock. In many cases, the application of heat is done through the use powerful xenon flash while thermal imaging is carried out using an infrared camera as illustrated in Figure 33. Application of heat flashes are followed by the recording of the surface temperature of the component using an infrared camera. Diffusion of heat waves is subject to reflection as they interfere with defects as illustrated in Figure 34. Defect free areas have a steady state surface temperature of $T_{\text {so }}$ as shown in Figure 34. Defective areas cause a reflection of waves back to the free surface and therefore the temperature increases by a small amount $T_{r}$ as shown in Figure 34 . The summation of $T_{s o}$ and $T_{r}$ is equal to the surface temperature of defective areas. Differences in surface temperatures along different locations may be used to identify damaged areas as shown in Figure 33 [32]. 


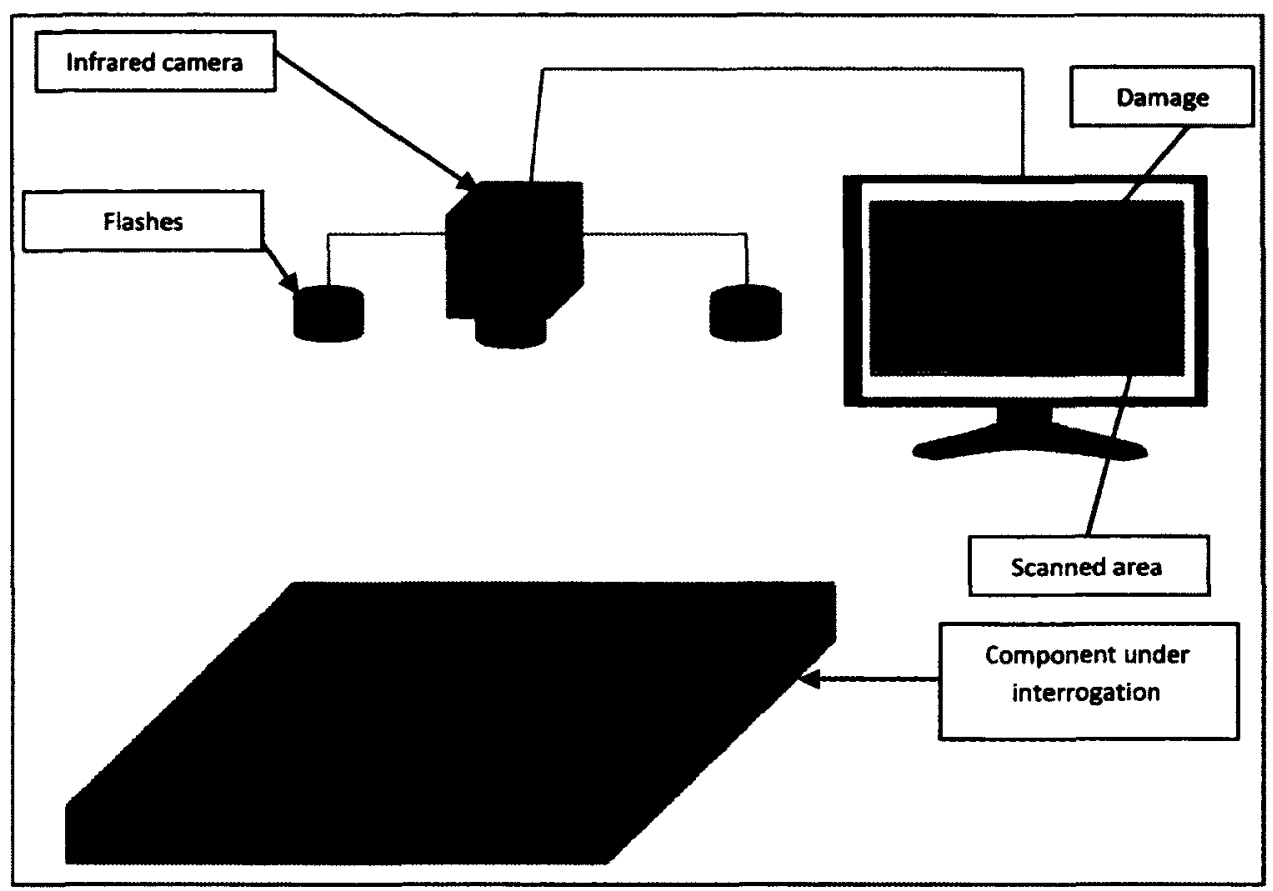

Figure 33: Pulsed Thermography Setup

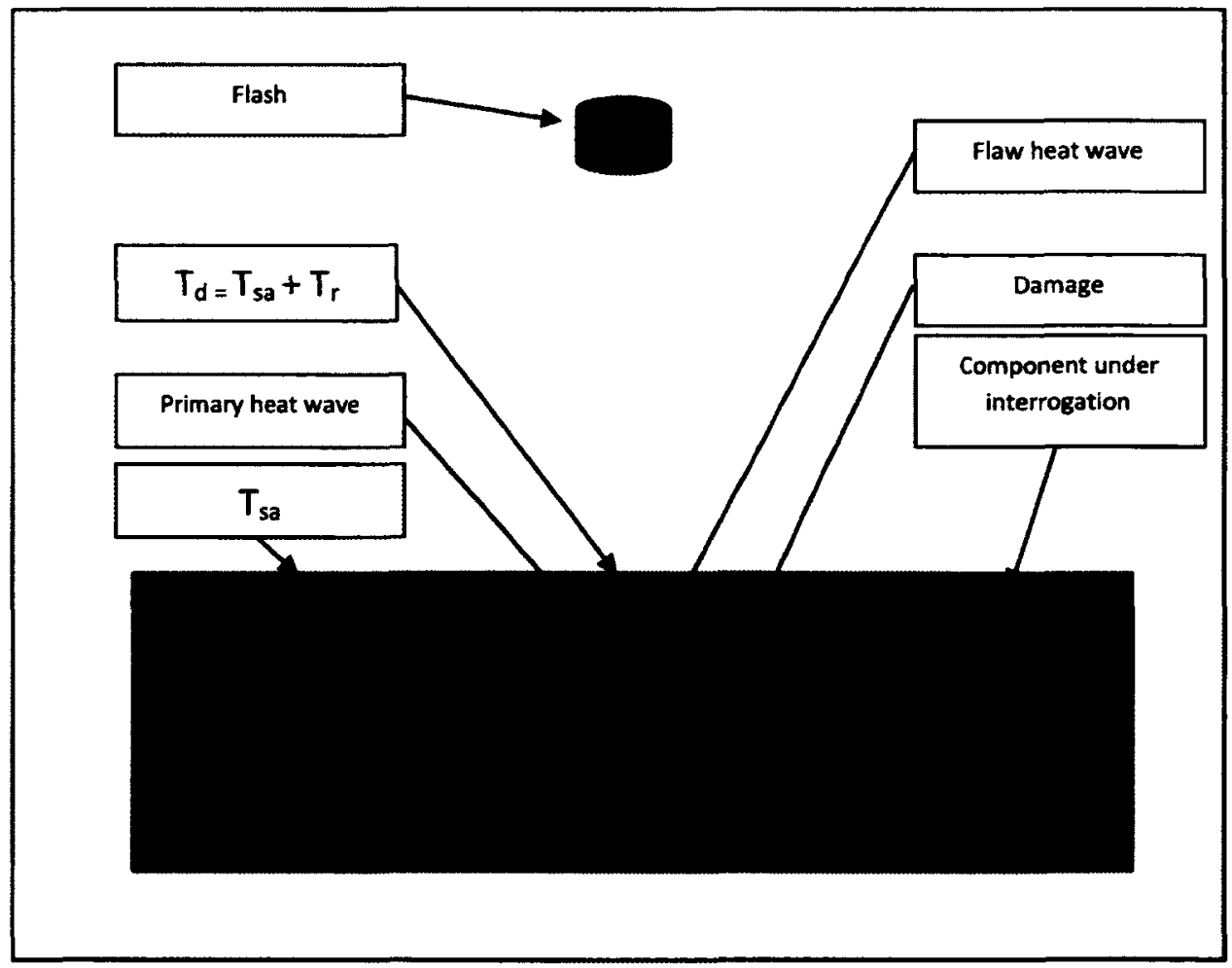

Figure 34: Pulsed Thermography Wave Propagation 
There are two forms of thermography, Pulse (PT) and Lock-in (LT) $[54,55,56]$. PT is an analysis for which the application of a single thermal unit pulse is applied and steady state temperature response is captured using the infrared camera. LT is an analysis for which the application of periodic heating is applied and the temperature profile is captured over a predefined time window. Energy input plots as well as temperature responses for both PT and LT have been presented within Figure 35.

PT requires less computational resources as compared to LT and it has the ability to detect defects at different depths. Damage detection is done by comparing surface temperatures of defective areas and defective-free areas. Two temperature profiles are captured using an infrared camera, $T_{s o}$ and $T_{d}$ as shown in Figure 36. $T_{s o}$ is the surface temperature of the defect areas while $T_{d}$ is the surface temperature of defective areas. If a difference of $T_{s a}$ and $T_{d}(\Delta T)$ exist as shown in Figure 36, then the system indicates damage. The surface temperature for defective areas is also affected by the depth of damage. Studies have shown that shallow damages will yield a higher $T_{d}$ while deeper damages will yield a lower $T_{d}$ [54]. Experimental analysis for a plate with multiple holes at different depths is shown in Figure 37(a); results of which show that $T_{d}$ is a function of defect depth. However, the $2.0 \mathrm{~mm}$ hole (top right corner) appears to have a higher $T_{d}$ than that of the $1.5 \mathrm{~mm}$ deep hole; this error is attributed to uneven heating of the surface. Figure $37(b)$ shows the transient state results of the surface temperature; from that image, it is clear that the surface is unevenly heated. Uneven heating is one of the major drawbacks to PT as it leads to inadequate defect depth detection. Uneven heating may be caused by a number of factors including wind and external lighting sources; this provides additional difficulty in defect detection. For that reason, LT becomes useful [55]. 


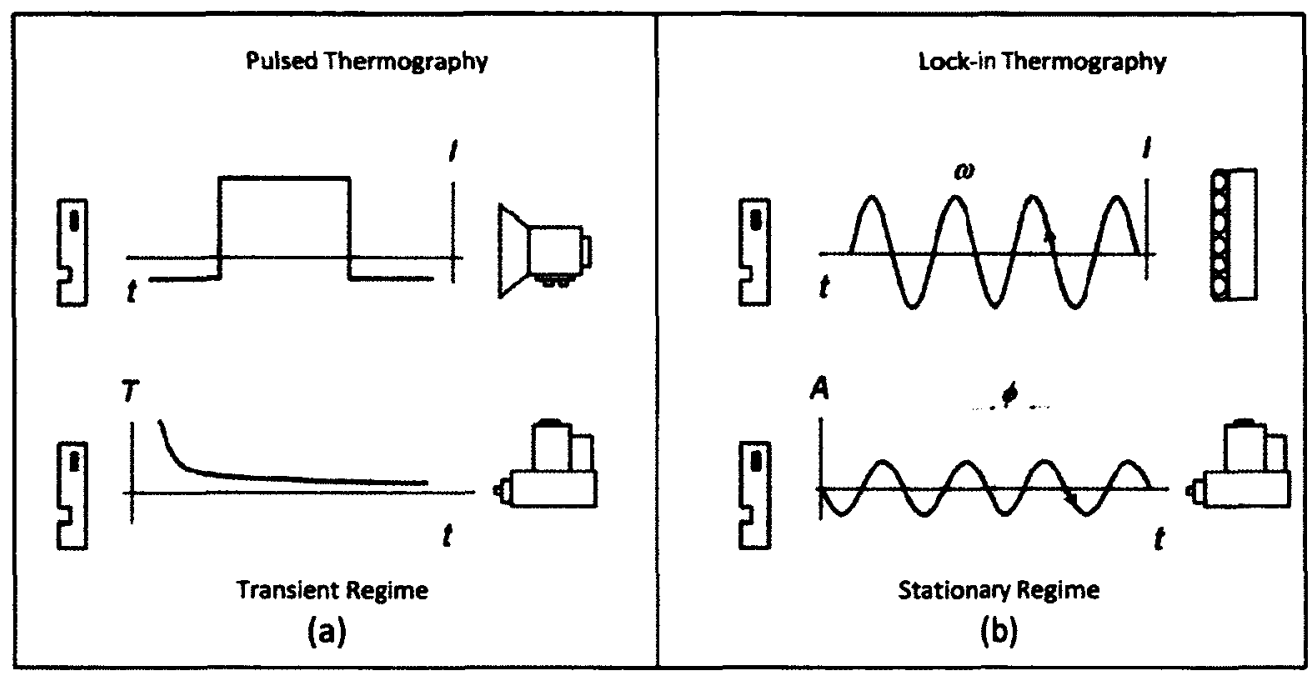

Figure 35: Heat Wave Propagation: (a) Pulsed Thermography, and (b) Lock-in Thermography [54]

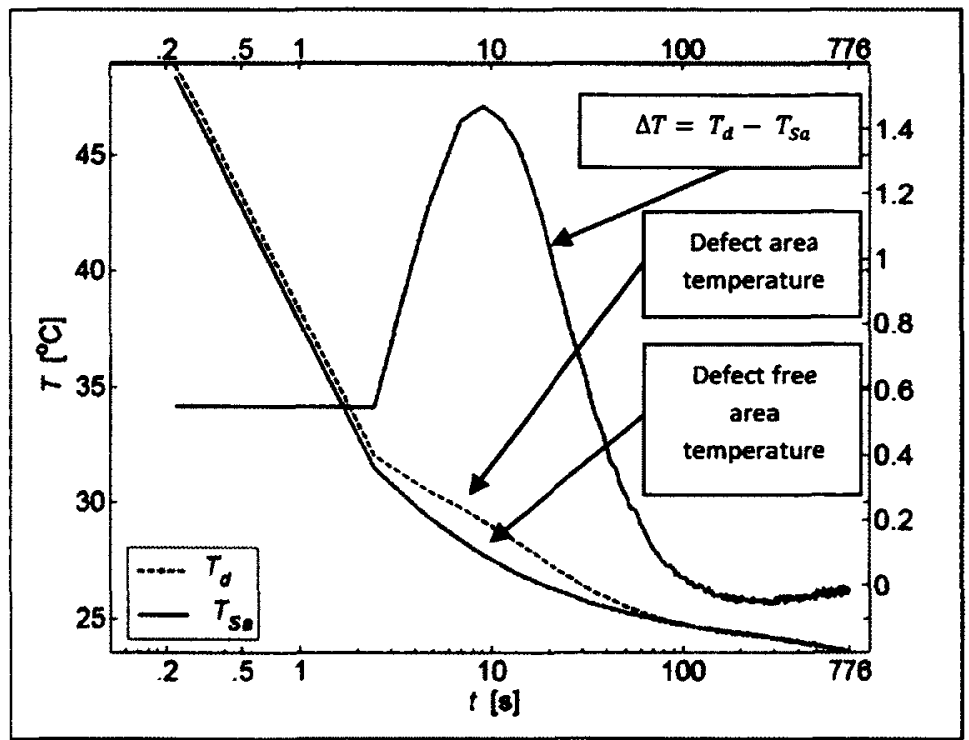

Figure 36: Temperature Profiles [54] 


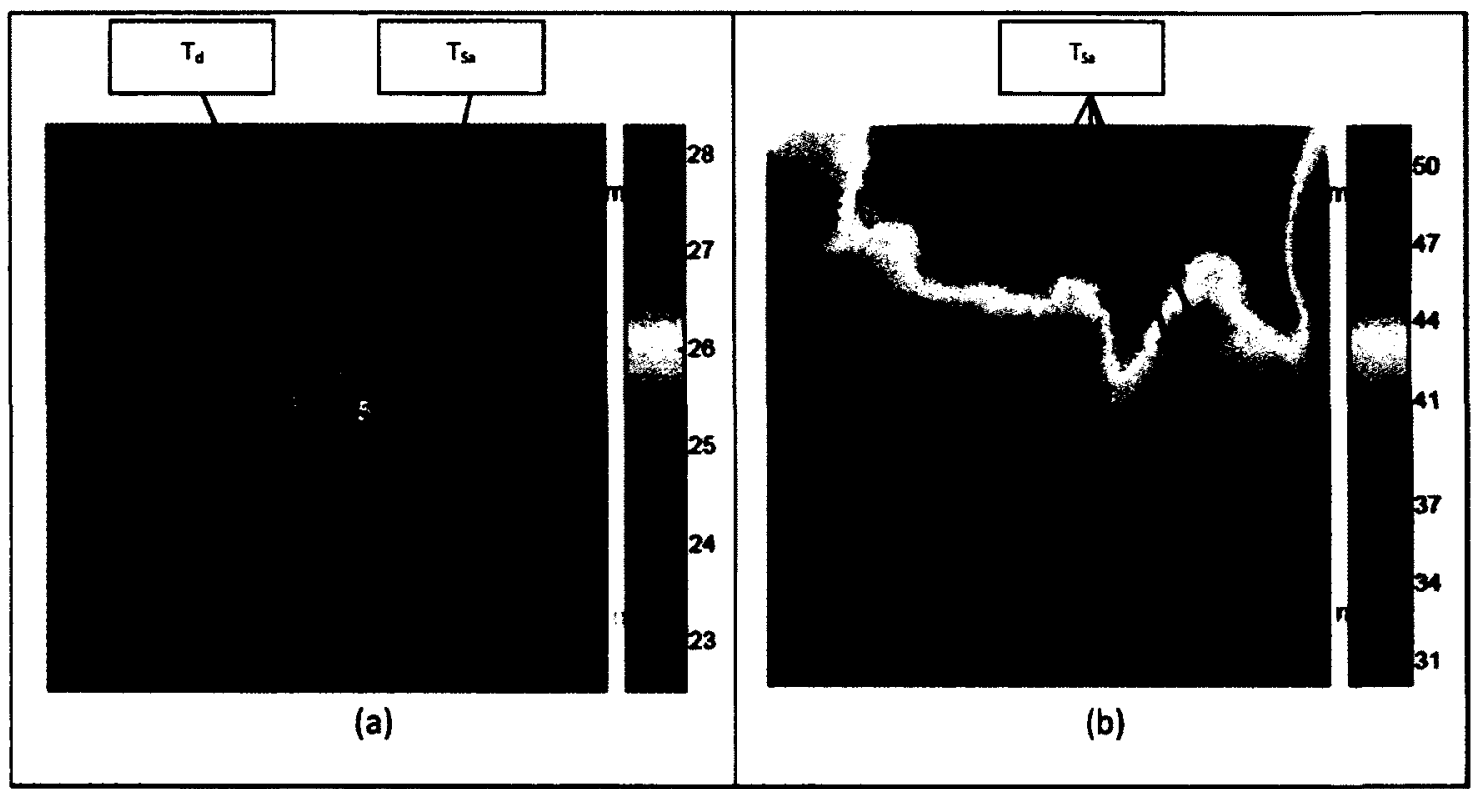

Figure 37: Example Thermography Images: (a) surface Thermal image after settling of temperature, and (b) Thermal image with uneven heating on [54]

LT is a thermography process which is carried out through the application of periodic heat waves. Heat wave analysis is dependent on phase shifts rather than just steady state surface temperatures. Recent research has shown that phase shift analysis is less susceptible to environmental interference [54]. However, the application of LT waves is done at specific frequencies. The application of single predefined frequencies limits the depth of inspection as depth of penetration is dependent on the frequency. Therefore, to scan for defects at various depths, the frequency must be changed for multiple scans; this can be very time consuming [54].

Both PT and LT have advantages and disadvantages. PT provides quick inspection as only one pulse is required for structure interrogation while LT requires various frequency applications. However, LT does provide environmental interference-free analysis and therefore is more accurate at the identification of damage. Both techniques may be combined to provide quick inspection with limited environmental interference; this is done through the use of Pulsed Phase Thermography (PPT) [54]. 
PPT is a combination of both PT and LT; it utilized heat application of PT and analysis methods of LT. Through the application of a single pulse as shown in Figure 38(a), multiple heat frequencies are introduced within the structure. The application of heat through the pulse may be approximated using infinite sinusoid frequencies as shown in Figure 38(b); this is done through the use of Fourier transforms. The analysis of such Discrete Fourier Transforms (DFT) for both amplitude and phase shifts is used for PPT and is an effective method for providing environment interference-free defect detection [54]. For this research PPT was used to validate the integrity of patches during testing.

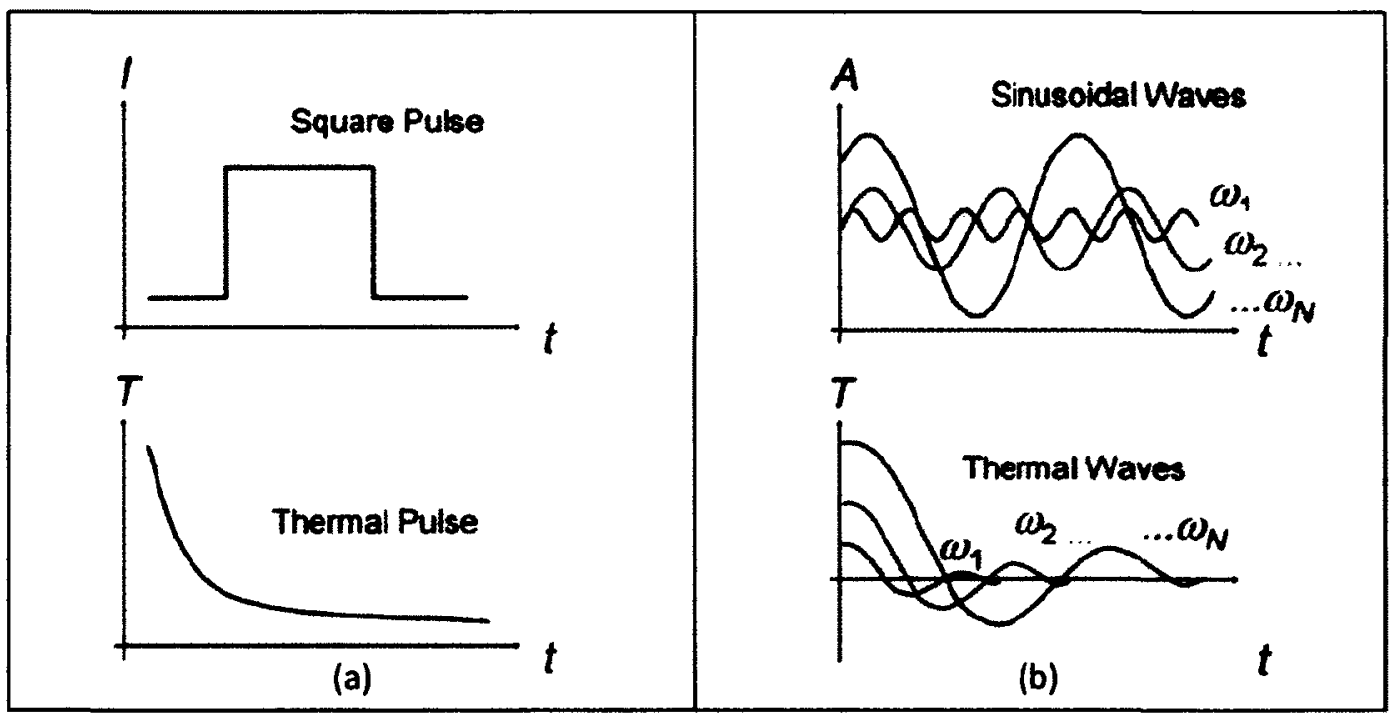

Figure 38: Pulsed Thermography: (a) Pulse within time domain, and (b) Pulse within frequency domain [54]

\section{$2.2 \quad$ Structural Health Monitoring (SHM) Systems}

There are many SHM systems that were discussed in Chapter 1. All systems may be used and tested for their effectiveness in the detection of disbond, however only 3 were selected for this study. The three selected SHM systems include Capacitance Disbond Detection Technique (CDDT), Surface Mountable Crack Detection System (SMCDS) and Acousto-Ultrasonic (AU). 
Other SHM technologies that have been described in section 1.2.2 have not been investigated in this study for the flowing reasons:

- Fibre Bragg Gratings (FBG) and Strain Gauges (SG): SG was amongst the first technologies to be utilized on patch bonded repairs [57]. Strain measurements have been tested by many research institutions around the world and there are many publications to support their findings. Research on the use of SG for SHM application concludes that this technology works well. However, to fully monitor the entire area of the patch, many strain gauges must be used. Strain gauges were not selected as previous research has shown their effectiveness. Also, due to limited access to FBG technology, this technology was not selected.

- Eddy Current (EC): EC technology works based on conduction properties of the components being tested. For this study, composite materials are being utilized. Unlike metallic materials, composites have no or very low conductive properties. For that reason, EC sensors were not selected.

- Comparative Vacuum monitoring (CVM): CVM is similar to SMCDS and therefore only one was chosen for this research. CVM is composed of small capillary tubes which are monitored for leaks. These leaks are caused by crack nucleation and growth underneath these capillary tubes. SMCDS is based on similar principle; however, rather than using capillary tubes, the system uses conductive paint with low voltage. Electrical flow is interrupted by the nucleation and growth of cracks through the conductive paint. Due to their similarities, only SMCDS was chosen.

- Acoustic Emission: Acoustic Emission sensors and data acquisition systems were not readily available. 


\subsubsection{Capacitance Disbond Detection Technique (CDDT)}

Capacitance Disbond Detection Technique (CDDT) has been utilized by the National Research Council Canada (NRC) for the detection of disbond of a patch from a metallic substrate. Tests at the NRC demonstrated that patch disbonds resulted in a drop of capacitance [48]. The following section describes the physics behind the CDDT.

\subsubsection{Basics of a capacitor}

A capacitor is a device that stores energy in the form of a static electrical field. Electromagnetic fields within a capacitor are created by allowing the formation of an electric potential difference. The potential difference is the result of negative and positive charge build-up along conductive plates which are separated by an insulating medium as show in Figure 39. Capacitance is the measure of the ability of a capacitor to store energy and is measured in Farads. Capacitance as shown in Equation 3 is the ratio of charge $(Q)$ build-up to the applied voltage (V). Capacitance can also be written in the form shown in Equation 4 [58,59].

$$
\begin{array}{cc}
C=\frac{Q}{V} & \text { Equation } 3 \\
C=\frac{K \varepsilon_{0} A}{d} & \text { Equation } 4
\end{array}
$$

Where:

- $K$ is the dielectric constant,

- $\varepsilon_{0}$ is the permittivity of free space and $\varepsilon_{0}=8.854 \times 10-12 \mathrm{~F} / \mathrm{m}$,

- $A$ is the area of each of the parallel plates as shown in Figure 39, and

- $d$ is the separation distance between the conductive plates; this is also the thickness of the isolating medium as shown in Figure 39. 


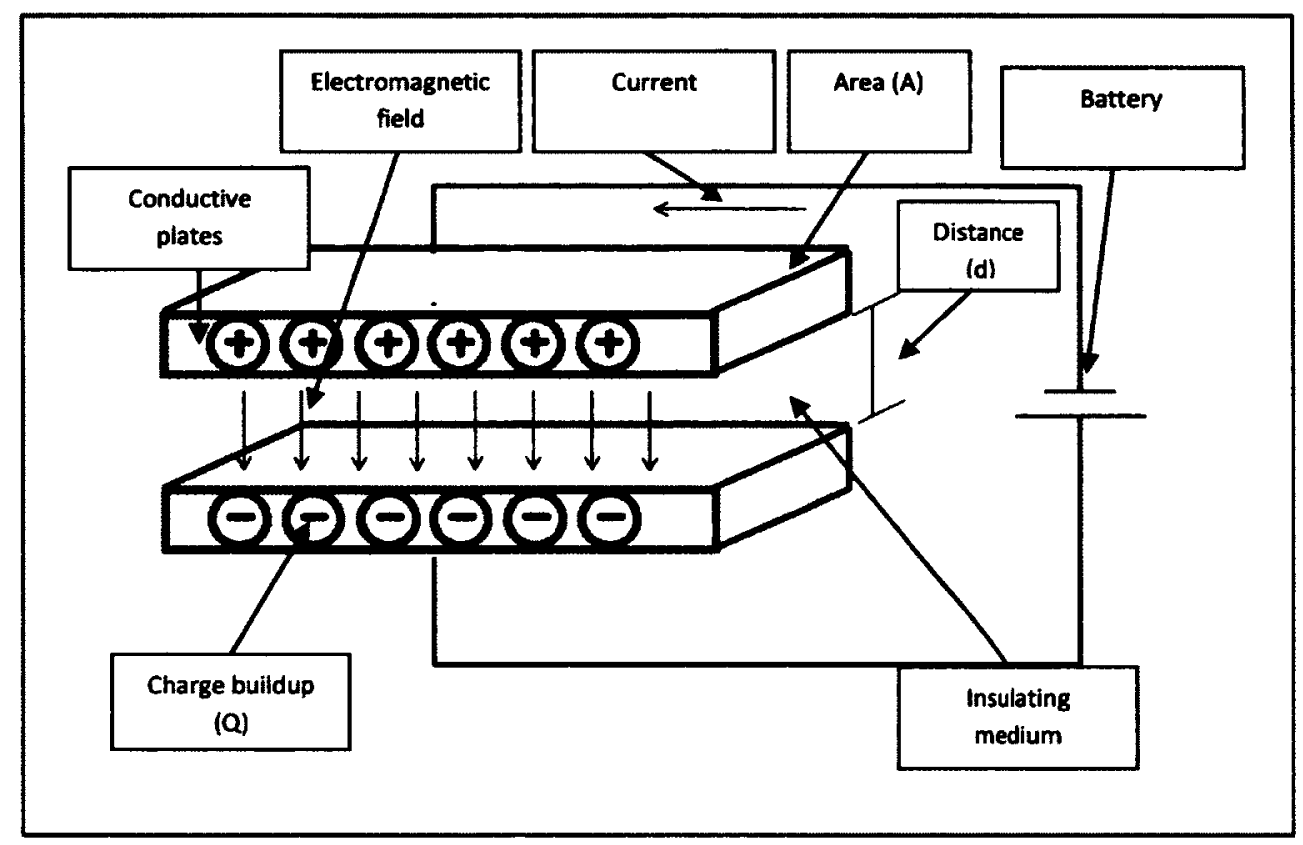

Figure 39: Basics of a Capacitor

In situations where the isolating layer remains unchanged, both $K$ and $\varepsilon_{o}$ from Equation 4 remain constant and therefore do not influence the capacitance of the system. In that specific case, the area of the conductive plates and the separation distance will dominate the energy carrying capacity of the capacitor.

Capacitor theory may be used for SHM of composite joints. This technology has the potential for the detection of adhesive failure within metallic-to-metallic, composite-to-metallic and composite-to-composite joints, provided that the components being joined are conductive. Patch bonded repairs are the most basic form of joints that may be monitored using CDDT, as the parallel plate capacitance equations hold. Figure $\mathbf{4 0}$ shows two joined components: a patch and a substrate which are bonded using a film adhesive. Fully bonded joint capacitance can be calculated using Equation 4: where $K$ is equal to that of the relative static permittivity of the adhesive and $d$ is equal to the thickness of the adhesive denoted by $d_{b}$ within the Magnification $A$ of Figure 40 [58,59]. Disbonded joint capacitance may be modeled using Equation 4; however, in this case, there exist two relative static permittivity values; one for the adhesive and one for 
the air-gap formed during disbond. In addition to the change in relative static permittivity, the distance between the patch and the substrate also changes due to the formation of the air-gap $\left(d_{d}\right)$ as shown in Magnification B of Figure 40. By measuring capacitance, the disbonded area may be quantified. By examining Equation 4, it is evident that disbond results in a decrease of capacitance. This is a due to an increase in distance between the two conductive surfaces as well as a reduction of the dielectric constant.

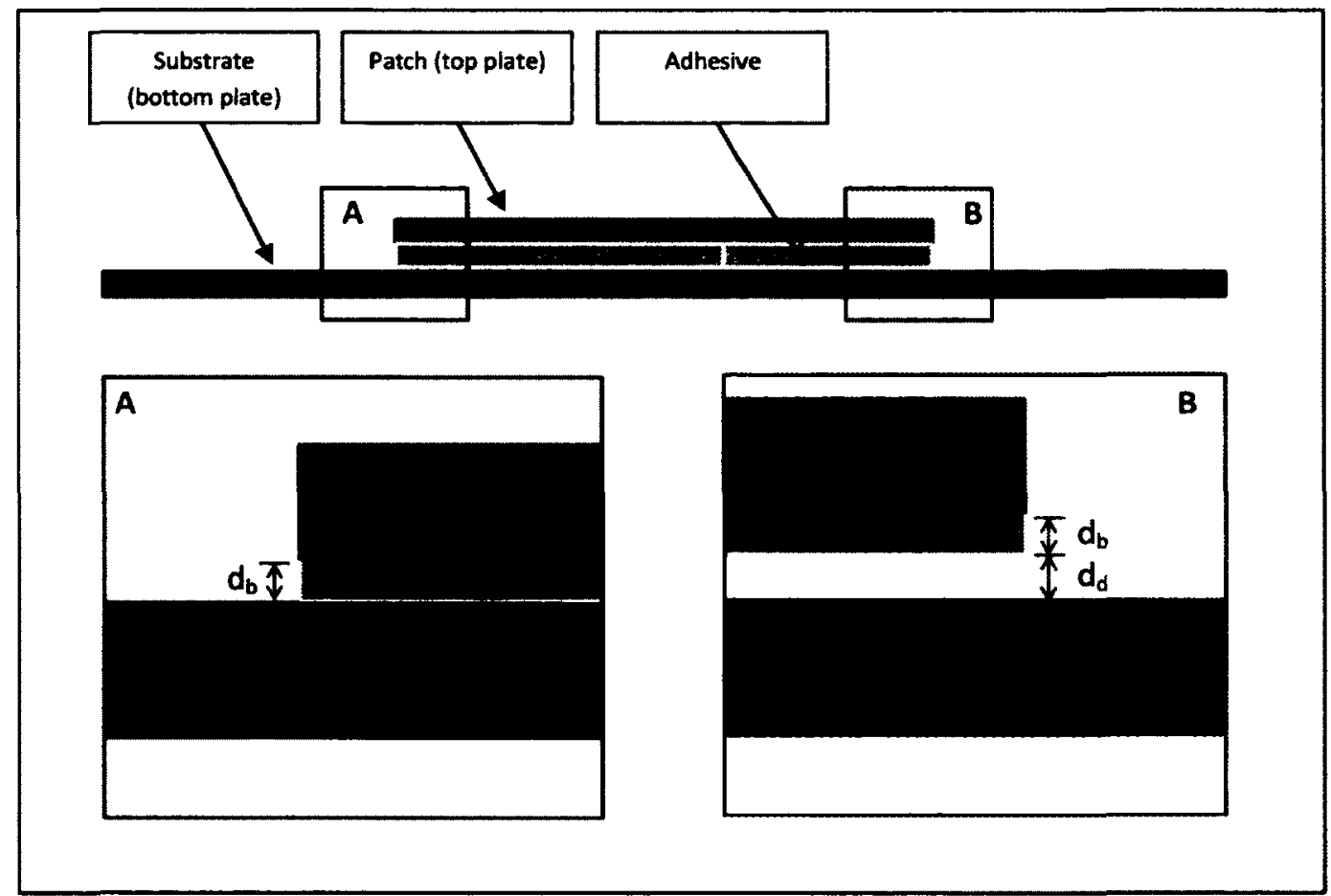

Figure 40: Patch Capacitor

\subsubsection{Surface Mountable Crack Detection System (SMCDS)}

Surface Mountable Crack Detection System (SMCDS) have been used at the National Research Council Canada (NRC) for detecting crack initiation and progression within metallic components. This technology is very limited to surface defects and therefore cannot detect all defects within composite structures, as many failure modes are caused by internal defects. This technology 
works by monitoring disruptions of electrical voltage through a circuit. Disruptions are a result of sensor breakage which is caused by crack propagation through the sensing element.

\subsubsection{Basics of Surface Mountable Crack Sensors (SMCS)}

Surface Mountable Crack Sensors (SMCS) which are used as part of the SMCDS system are conductive paint on sensors that act as open-close switches of a conductive circuit as illustrated in Figure 41. SMCS are installed onto areas where the probability of defect initiation is high (i.e. highly stressed areas). SMCS are wired to a circuit and low current is allowed to flow through; undamaged SMCS will not hinder electron flow as the SMCS is a silver based conductive paint as shown in Figure 41(a). As defects initiate and grow, the SMCS are subject to breakage as the silver based paint is brittle. Once broken, the SMCS act as an open switch as shown in Figure 41(b). By monitoring the voltage and or current of the circuit, surface defects may be detected.

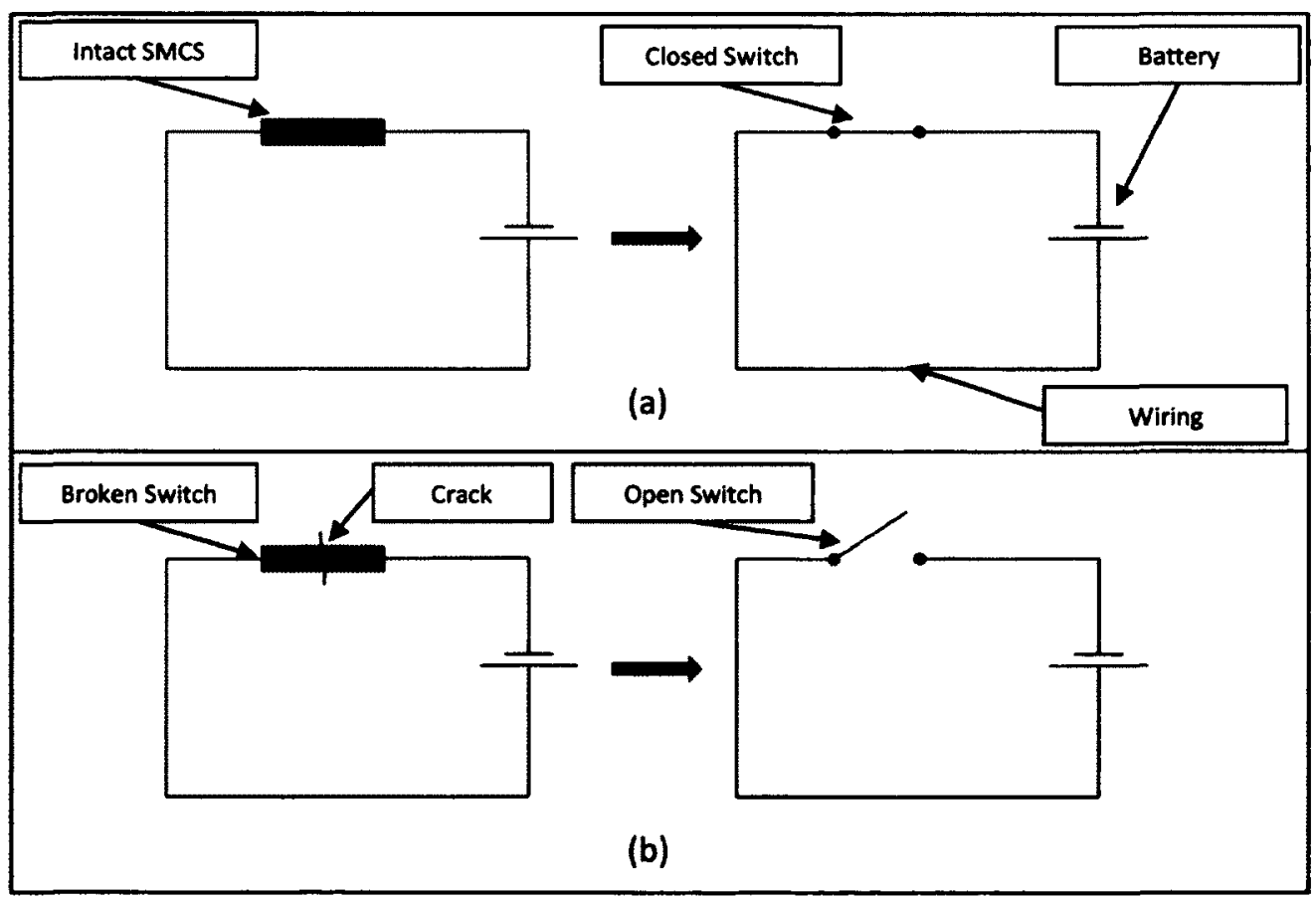

Figure 41: SMCS Circuits: (a) Intact SMCS acting as a closed switch and (b) cracked SMCS acting as an open switch 
In some cases, the SMCS is installed on components that are conductive; these may be of metallic or of composite composition. Conductive components must be insulated from the SMCS circuit so that accurate voltage and or current measurements may be recorded. An insulating medium, such as a ceramic based coating, is applied prior to placement of the conductive paint as shown in Figure 42. In addition to insulation, the ceramic based coating is brittle so that cracks are allowed to propagate through the ceramic paint and through the conductive paint. After application of the ceramic and conductive paints, a protective layer may be painted on top to protect them from the environment.

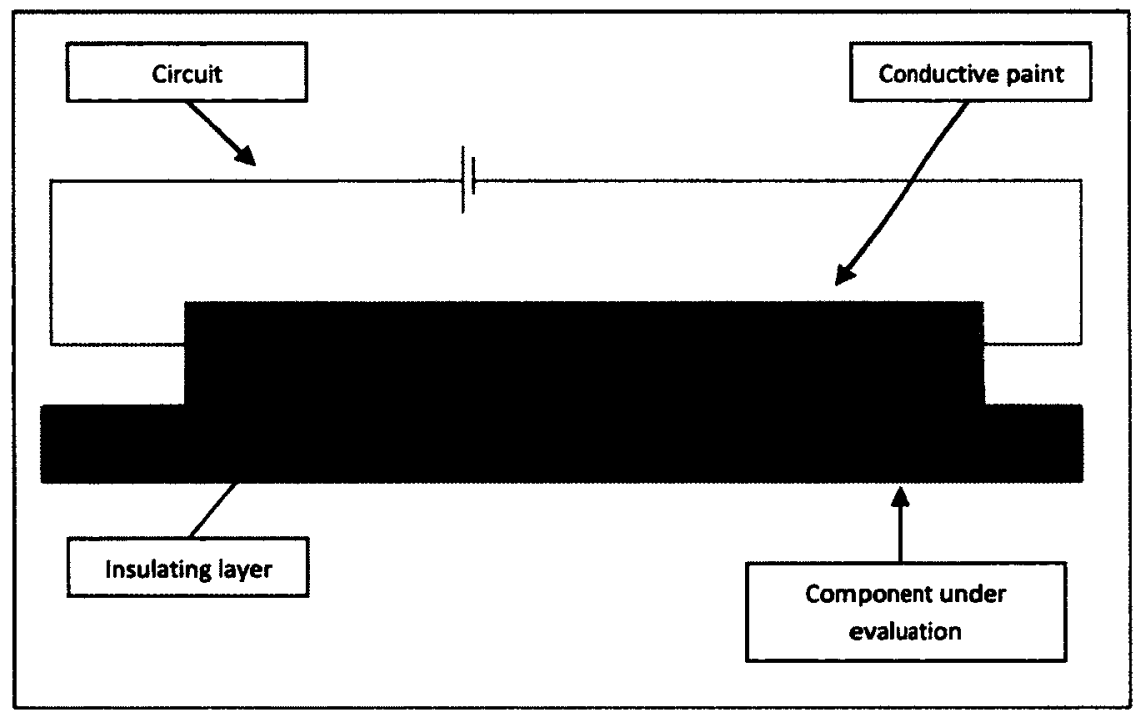

Figure 42: SMCS Composition

Detection of crack nucleation is done through the use of a single sensor; however, crack sizes may also be monitored through the use of multiple sensors placed in parallel and independently monitored as shown in Figure 43. 


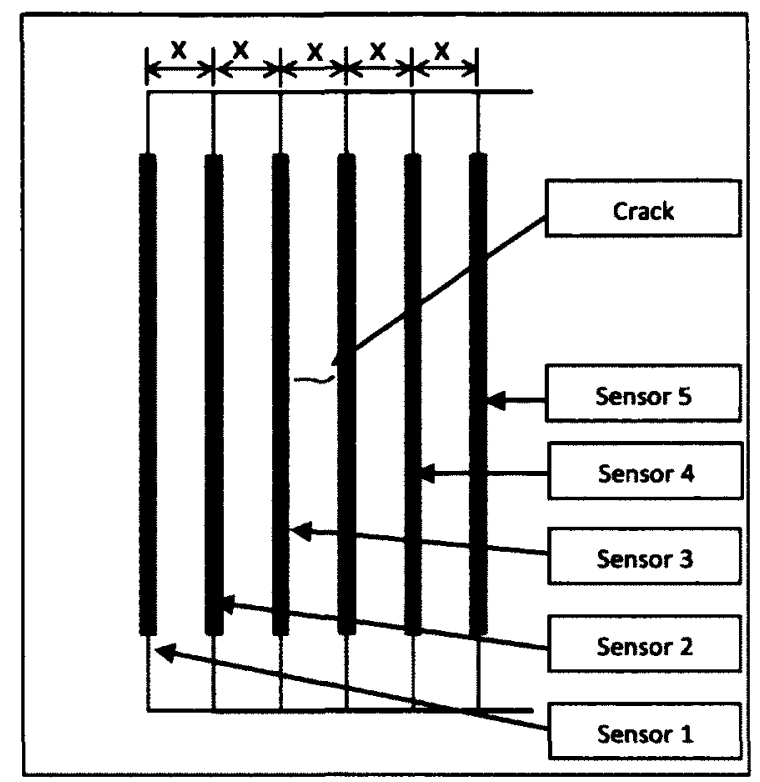

Figure 43: SMCS Placement

\subsubsection{Acousto-Ultrasonic (AU)}

Acousto-Ultrasonic is a method by which the integrity of a structure is investigated through the propagation of ultrasonic waves. All materials have specific properties that change with the introduction of damage. Wave propagation through materials is highly dependent on the material's properties; changes in such properties leads to a change in signal propagation and their responses. Changes of such signals may be correlated to damage $[33,60,61]$.

There are many types of ultrasonic waves that exist within structures, all of which are classified as elastic waves. One small subset of such waves are Lamb waves. Lamb waves are surface guided waves that propagate over large distances and are less susceptible to large attenuation, even in composite materials [32]. Lamb waves scatter as well as undergo modal conversion in the presence of damage; this makes them suitable for damage detection. Analysis of lamb waves have the capability of detecting disbonding, corrosion, cracking, holes, delamination, broken fibres, resin cracks, inclusions and moisture ingression [33,62]. However, Lamb waves exist in multiple modes, wave reflection and dispersion of such modes off boundaries makes it 
difficult to interpret damage as algorithms are frequency dependent $[33,63]$. In composites, there exist two phases: a fibre and a matrix; Lamb waves interact with these two phases, resulting in lots of signal dispersion and reflection, thus making it difficult to analyze the signals.

\subsubsection{Basics of Lamb waves}

Lamb waves (LW) are known as guided waves, which are bounded between two free surfaces. $\mathrm{LW}$ are elastic perturbations propagating within thin structures. The propagation of $\mathrm{LW}$ in a thin structure along with the co-ordinate system is illustrated in Figure 44 . The motion of the particles in LW are in the direction of wave propagation and perpendicular to it. LW are superposition of pressure waves ( $\mathrm{P}$-waves) and shear vertical waves (SV-waves). P-waves are generated by applying normal forces along the $x_{1}$ direction [Figure 45(a)]; whereas, SV-waves are generated by applying shear forces along the $x_{3}$ direction [Figure $45(b)$ ]. The combination of $P$ and SV waves generate $\mathrm{LW}$, which propagate in two different and independent modes, known as symmetric $\left(S_{n}\right)$ and anti-symmetric $\left(A_{n}\right) .[33,63,64]$

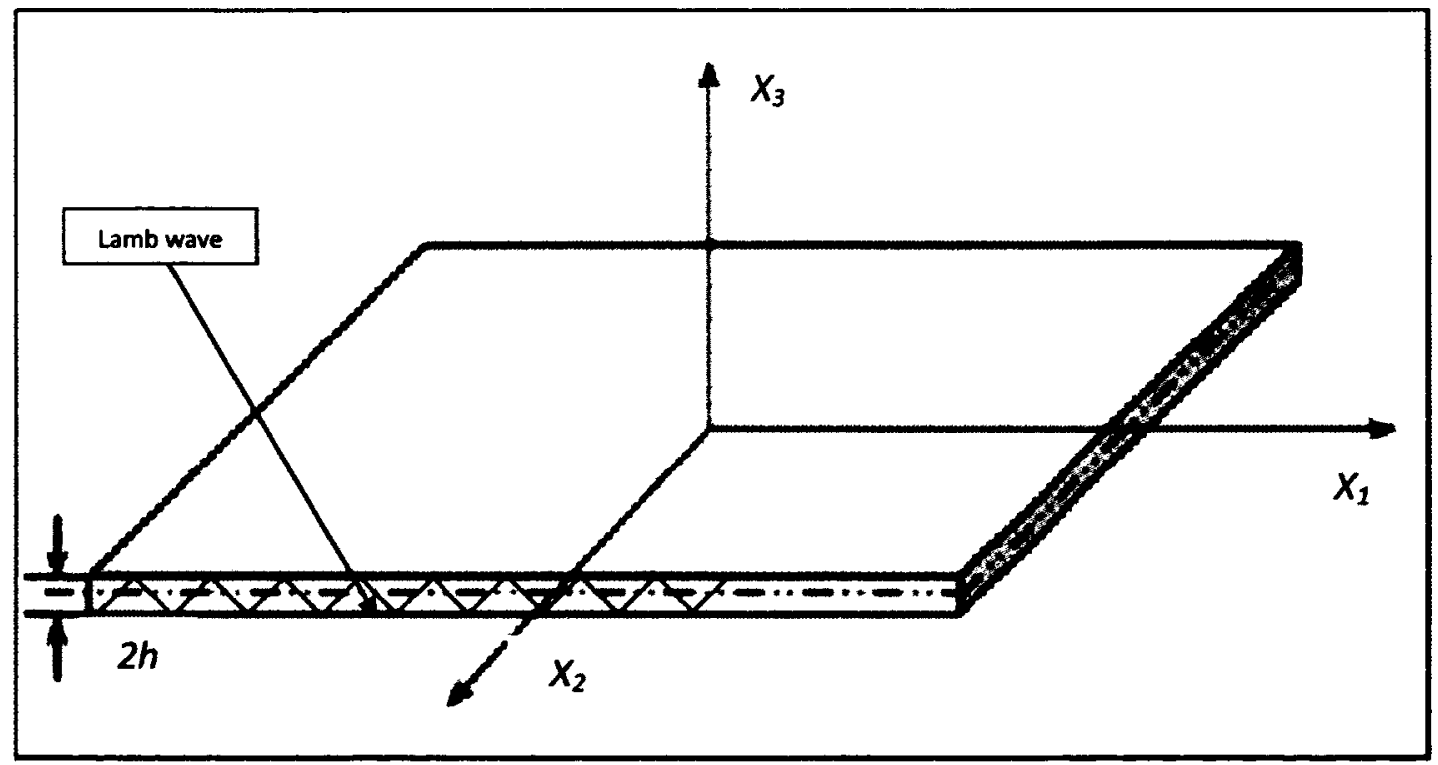

Figure 44: Co-ordinate System [33] 


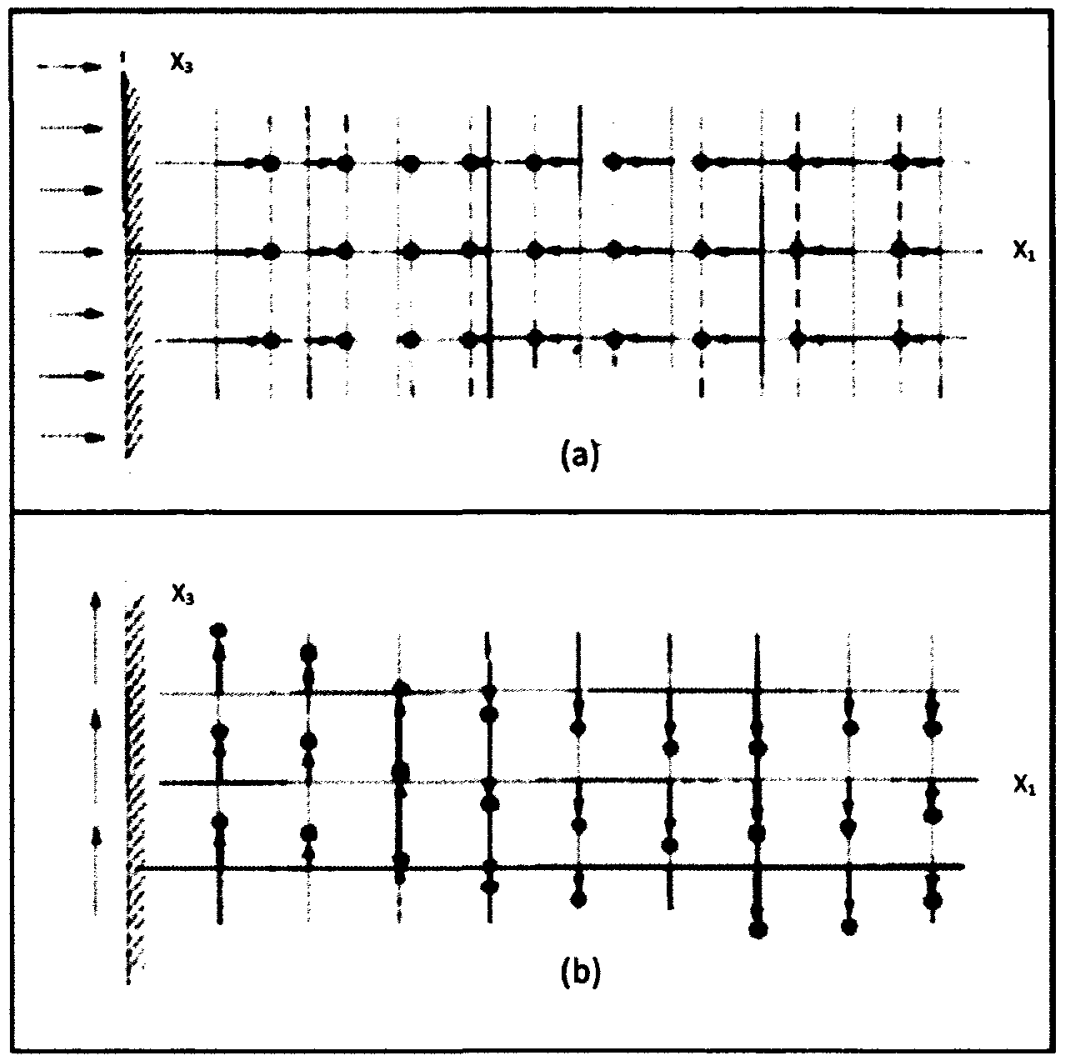

Figure 45: Particle Motion within a Solid Medium: (a) particles subject to pressure force, and (b) particles subject to shear force [64]

Note: Detail derivation of LW equations is beyond the scope of this thesis; therefore only the important steps for deriving the LW equations for isotropic materials are discussed. Einstein's summation convention for tensor notation is used to show the derivation of $\mathrm{LW}$ equations. All the following equations have been obtained through two primary sources $[33,64]$.

The following steps are generally taken to derive the LW equations [64]:

Consider equation of motion using Newton's second law, which gives:

$$
\sum F_{i}=m a_{i}
$$

Equation 5

Where:

- $F$ is the force,

- $m$ is the mass, 
- $a$ is the acceleration, and

- $i=1,2,3$

Consider equation of motion using Newton's second law, which gives:

$$
F_{N}=\sigma A ; F_{T}=\tau A
$$

Equation 6

Where

- $\sigma$ is the stress,

- $A$ is the area,

- $\tau$ is the shear stress, and

- $\quad N$ and $T$ are subscripts denoting Normal and Tangential directions respectively

For a 3-D case, stresses can be written in the matrix form as:

$$
T_{i j}=\left[\begin{array}{lll}
\sigma_{x x} & \tau_{x y} & \tau_{x z} \\
\tau_{x y} & \sigma_{y y} & \tau_{y z} \\
\tau_{x z} & \tau_{y z} & \sigma_{z z}
\end{array}\right]
$$

Equation 7

Stresses can be related in tensor form in terms of strains as:

$$
T_{i j}=\lambda\left(\varepsilon_{11}+\varepsilon_{22}+\varepsilon_{33}\right) \delta_{i j}+2 G \varepsilon_{i j}
$$

Equation 8

Where

- $T_{i j}$ is the stress,

- $i=1,2,3$

- $j=1,2,3$

- $\varepsilon_{i j}$ is the strain,

- $\lambda$ is the lame constant and denoted by Equation 9

- $G$ is the shear modulus, and

- $\delta_{i j}$ is the Kronecker delta and denoted by Equation 10

$$
\lambda=\frac{v E}{(1+v)(1-2 v)}
$$

Where

- $E$ is Young's modulus, and

- $v$ is Possion's ratio 


$$
\delta_{i j}=\left\{\begin{array}{l}
1 \text { if } i=j \\
0 \text { if } i \neq j
\end{array}\right.
$$

Equation 10

Strains can be related to displacement using:

$$
\varepsilon_{i j}=\frac{1}{2}\left(\frac{\partial u_{i}}{\partial x_{j}}+\frac{\partial u_{j}}{\partial x_{i}}\right)
$$

Equation 11

Where:

- $u$ is the displacement, and

- $x$ is the direction

To understand LW, one must first examine the motion of particles within a material. Equation 5 through Equation 10 will be used in the following steps to evaluate how particles behave in the presence of applied forces.

Consider an infinitesimal element as shown in Figure 46: 


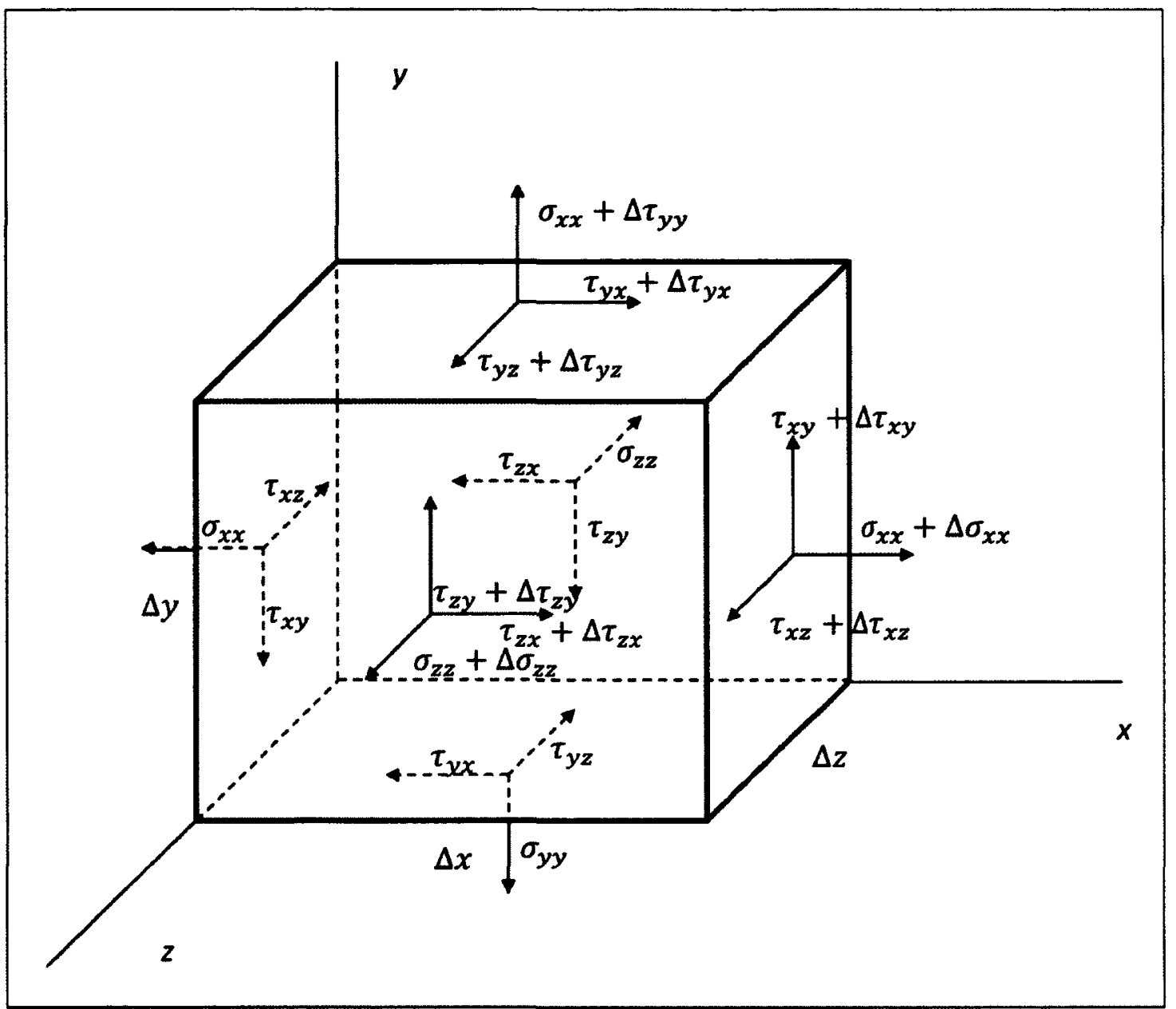

Figure 46: Stress Distribution on a Small Element

Equation of motion (Equation 5) for this element in the $\mathrm{x}$-direction can be written as:

$$
\begin{aligned}
& \left(\sigma_{x x}+\Delta \sigma_{x x}\right) \Delta y \Delta z-\Delta \sigma_{x x} \Delta y \Delta z+\left(\tau_{y x}+\Delta \tau_{y x}\right) \Delta x \Delta z \\
& -\Delta \tau_{y x} \Delta x \Delta z+\left(\tau_{z x}+\Delta \tau_{x x}\right) \Delta x \Delta y-\Delta \tau_{z x} \Delta x \Delta y=\rho \Delta x \Delta y \Delta z \frac{\partial^{2} u_{1}}{\partial t^{2}}
\end{aligned}
$$

Equation 12

Dividing (Equation 8) by $\Delta x \Delta y \Delta z$ and taking the limit as $\Delta x, \Delta y$ and $\Delta z$ goes to zero, gives:

$$
\frac{\partial \sigma_{x x}}{\partial x}+\frac{\partial \tau_{y x}}{\partial y}+\frac{\partial \tau_{z x}}{\partial z}=\rho \frac{\partial^{2} u_{1}}{\partial t^{2}}
$$


Where:

- $\rho$ is the material density

Equation of motion for $y$ - and z-direction can be generated in a similar fashion

Substituting the stress (Equation 8 ) into Equation 13 yields:

$$
\begin{aligned}
& \frac{\partial}{\partial x_{1}}\left[\lambda\left(\varepsilon_{11}+\varepsilon_{22}+\varepsilon_{33}\right) \delta_{i j}+2 G \varepsilon_{i j}\right]+\frac{\partial}{\partial x_{2}}\left[\lambda\left(\varepsilon_{11}+\varepsilon_{22}+\varepsilon_{33}\right) \delta_{i j}+2 G \varepsilon_{i j}\right] \\
& +\frac{\partial}{\partial x_{3}}\left[\lambda\left(\varepsilon_{11}+\varepsilon_{22}+\varepsilon_{33}\right) \delta_{i j}+2 G \varepsilon_{i j}\right]=\rho \frac{\partial^{2} u_{i}}{\partial t^{2}}
\end{aligned}
$$

Through manipulation, Equation 14 can be further reduced into Equation 15 and consequently into Equation 16.

$$
\begin{gathered}
\left(\frac{\partial}{\partial x_{1}}+\frac{\partial}{\partial x_{2}}+\frac{\partial}{\partial x_{3}}\right)\left[\lambda\left(\varepsilon_{11}+\varepsilon_{22}+\varepsilon_{33}\right) \delta_{i j}+2 G \varepsilon_{i j}\right]=\rho \frac{\partial^{2} u_{i}}{\partial t^{2}} \\
\nabla\left(\lambda\left(\varepsilon_{11}+\varepsilon_{22}+\varepsilon_{33}\right) \delta_{i j}+2 G \varepsilon_{i j}\right)=\rho \frac{\partial^{2} u_{i}}{\partial t^{2}}
\end{gathered}
$$

The equation of motion (Equation 16) can be written using Equation 11 in terms of displacement as:

$$
(\lambda+G) \frac{\partial^{2} u_{i}}{\partial x_{j} \partial x_{j}}+G \frac{\partial^{2} u_{i}}{\partial x_{j} \partial x_{j}}=\rho \frac{\partial^{2} u_{i}}{\partial t^{2}}
$$

From Equation 17, the general longitudinal wave equation can be written as:

$$
\frac{\partial^{2} u_{1}}{\partial x_{1}^{2}}=\frac{1}{c_{L}^{2}} \frac{\partial^{2} u_{1}}{\partial t^{2}}
$$

Equation 18

Where:

- $\quad c_{L}=\mathrm{P}$-wave speed and is given by Equation 19

$$
c_{L}=\left(\frac{(\lambda+2 G)}{\rho}\right)^{1 / 2}
$$

Similarly, the general transverse wave equation can be written as: 


$$
\frac{\partial^{2} u_{2}}{\partial x_{1}^{2}}=\frac{1}{c_{T}^{2}} \frac{\partial^{2} u_{2}}{\partial t^{2}}
$$

Equation 20

Where:

- $c_{T}=$ shear or S-wave velocity and is given by Equation 21:

$$
c_{T}=\left(\frac{G}{\rho}\right)^{1 / 2}
$$

Equation 21

Lamb wave equations are generated assuming that motion depends only on $x_{1}$ and $x_{3}$ directions.

Using the Helmholtz decomposition in which a three-dimensional displacement vector can be decomposed into a sum of curl-free and divergence-free vector field, the displacement vector can be written as:

$$
\vec{u}=\nabla \phi+\nabla \times \vec{\psi}
$$

Equation 22

Where:

- $\phi$ is the scalar function and

- $\psi$ is the vector function. Both of which will be computed at later stages within the derivation.

The displacement vector $\vec{u}$, can be expanded as:

$$
\vec{u}=\frac{\partial \phi}{\partial x_{1}}+\frac{\partial \phi}{\partial x_{3}}-\frac{\partial \psi_{2}}{\partial x_{3}} \vec{e}_{1}+\frac{\partial \psi_{2}}{\partial x_{1}} \vec{e}_{2}
$$

Equation 23 can be simplified in terms of individual displacements as:

$$
\begin{aligned}
& u_{1}=\frac{\partial \phi}{\partial x_{1}}+\frac{\partial \psi_{2}}{\partial x_{3}} \\
& u_{2}=0 \\
& u_{3}=\frac{\partial \phi}{\partial x_{3}}-\frac{\partial \psi_{2}}{\partial x_{1}}
\end{aligned}
$$

The governing longitudinal wave equation (Equation 18) in terms $\phi$ can be written as: 


$$
\frac{\partial^{2} \phi}{\partial x_{1}^{2}}+\frac{\partial^{2} \phi}{\partial x_{3}^{2}}=\frac{1}{c_{L}^{2}} \frac{\partial^{2} \phi}{\partial t^{2}}
$$

Equation 25

Similarly, the governing transverse wave equation (Equation 20 ) in terms of $\psi$ can be written as:

$$
\frac{\partial^{2} \psi}{\partial x_{1}^{2}}+\frac{\partial^{2} \psi}{\partial x_{3}^{2}}=\frac{1}{c_{T}^{2}} \frac{\partial^{2} \psi}{\partial t^{2}}
$$

Equation 26

Solutions to the scalar and vector functions may be obtained by assuming the following functions:

$$
\begin{gathered}
\phi=\left[A_{1} \sin \left(p x_{3}\right)+A_{2} \cos \left(p x_{3}\right)\right] e^{i\left(k k_{1}-a v\right)} \\
\psi=\left[B_{1} \sin \left(q x_{3}\right)+B_{2} \cos \left(q x_{3}\right)\right] e^{i\left(k x_{1}-a v\right)}
\end{gathered}
$$

Equation 28

Where:

- $\omega$ is the angular frequency,

- $t$ is time,

- $k$ is the circular wave number,

- $A_{1}, A_{2}, B_{2}, B_{2}$ are all constants determined by the boundary conditions, and

- $\quad p$ and $q$ are equal to Equation 29 and Equation 30, respectively.

$$
\begin{aligned}
& p^{2}=\left(\frac{\omega^{2}}{c_{L}^{2}}-k^{2}\right) \\
& q^{2}=\left(\frac{\omega^{2}}{c_{T}^{2}}-k^{2}\right)
\end{aligned}
$$

Equation 29

Equation 30

Substituting Equation 27 and Equation 28 into Equation 24, displacements can be written as:

$$
\begin{aligned}
& u_{1}=i k\left[A_{1} \sin \left(p x_{3}\right)+A_{2} \cos \left(p x_{3}\right)\right] e^{i\left(k x_{1}-\alpha x\right)}+\left[q B_{1} \cos \left(q x_{3}\right)-q B_{2} \sin \left(q x_{3}\right)\right] e^{i\left(k x_{1}-a x\right)} \quad \text { Equation } 31 \\
& u_{3}=\left[p A_{1} \cos \left(p x_{3}\right)-p A_{2} \sin \left(p x_{3}\right)\right] e^{i\left(\alpha x-k x_{1}\right)}-i k\left[B_{1} \sin \left(q x_{3}\right)+B_{2} \cos \left(q x_{3}\right)\right] e^{i\left(\alpha x-k x_{1}\right)}
\end{aligned}
$$

Substituting the displacements (Equation 31 ) into Equation 8 , the stresses can be written as:

$$
\sigma_{31}=G e^{i\left(k k_{1}-\omega x\right)}\left(\begin{array}{l}
2 i k\left[p A_{1} \cos \left(p x_{3}\right)-p A_{2} \sin \left(p x_{3}\right)\right]+k^{2}\left[B_{1} \sin \left(q x_{3}\right)+B_{2} \cos \left(q x_{3}\right)\right]+ \\
{\left[-q^{2} B_{1} \sin \left(q x_{3}\right)-q^{2} B_{2} \cos \left(q x_{3}\right)\right]}
\end{array}\right)
$$




$$
\sigma_{33}=e^{i\left(k_{1}-\infty\right)}\left[\begin{array}{c}
\lambda\left(-k^{2}\left[A_{1} \sin \left(p x_{3}\right)+A_{2} \cos \left(p x_{3}\right)\right]+\left[-p^{2} A_{1} \sin \left(p x_{3}\right)-p^{2} A_{2} \cos \left(p x_{3}\right)\right]\right) \\
+2 G\left(\left[-p^{2} A_{1} \sin \left(p x_{3}\right)-p^{2} A_{2} \cos \left(p x_{3}\right)\right]-i k\left[q B_{1} \cos \left(q x_{3}\right)-q B_{2} \sin \left(q x_{3}\right)\right]\right)
\end{array}\right]
$$

The motion of particles within the structure are dependent on both sine and cosine terms; therefore each equation yields two modes of wave propagation. In the $x_{1}$ direction, motion is symmetric for only cosine terms and anti-symmetric for sine terms. In the $x_{3}$ direction, motion is symmetric for sine terms and anti-symmetric for cosine terms. Symmetric and anti-symmetric motion of particles and waves are shown in Figure 47 [64].

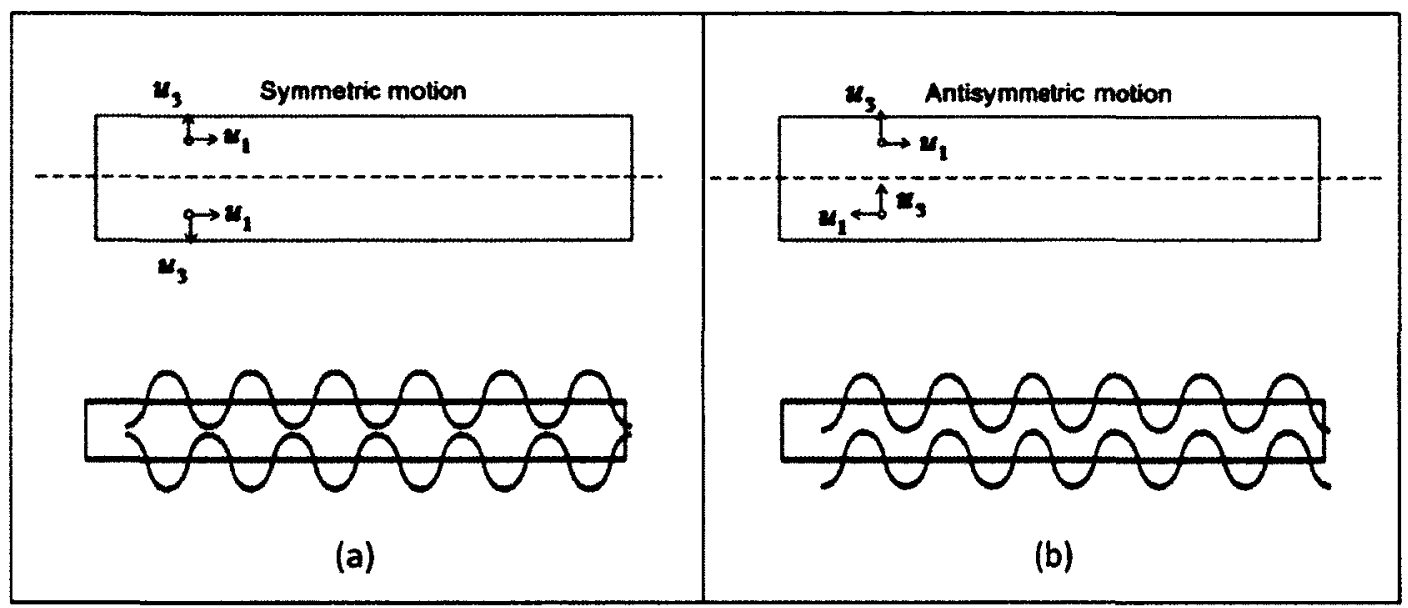

Figure 47: Lamb Wave Propagation: (a) Symmetric mode and (b) Anti-symmetric mode

\subsection{Symmetric and anti-symmetric solutions}

Removal of sine terms from $u_{1}$ and $\sigma_{33}$ and cosine terms from $u_{3}$ and $\sigma_{31}$ yields the symmetric equations below. The system of equations may be solved by applying the boundary condition at the surface of the material $\left(x_{3}= \pm h\right)$ where $\sigma_{31}=\sigma_{33}=0[64]$.

$$
\begin{array}{ll}
\phi=A_{2} \cos \left(p x_{3}\right) & \text { Equation } 33 \\
\psi=B_{1} \sin \left(q x_{3}\right) & \text { Equation } 34
\end{array}
$$




$$
\begin{array}{cc}
u_{1}=\left[q B_{1} \cos \left(q x_{3}\right)+i k A_{2} \cos p x_{3}\right] & \text { Equation } 35 \\
u_{3}=-\left[i k B_{1} \sin \left(q x_{3}\right)+p A_{2} \sin \left(p x_{3}\right)\right] & \text { Equation } 36 \\
\sigma_{31}=G\left[-2 i k p A_{2} \sin \left(p x_{3}\right)+\left(k^{2}-q^{2}\right) B_{1} \sin \left(q x_{3}\right)\right] & \text { Equation } 37 \\
\sigma_{33}=-\lambda k^{2} A_{2} \cos \left(p x_{3}\right)-\lambda p^{2} A_{2} \cos \left(p x_{3}\right) & \text { Equation } 38 \\
-2 G p^{2} A_{2} \cos \left(p x_{3}\right)-2 G i k q B_{1} \cos \left(q x_{3}\right) &
\end{array}
$$

By equating Equation 37 and Equation 38 to zero as per the surface boundary conditions, a solution for the equations may be identified. The non-trivial solution for the system of equations then becomes Equation 39 [64].

$$
\frac{\tan (q h)}{\tan (p h)}=\frac{-4 k^{2} p q}{\left(q^{2}-k^{2}\right)}
$$

Equation 39

Using the same approach and same surface boundary conditions, the anti-symmetric solution to the following equations may be obtained [64].

$$
\begin{array}{cc}
\phi=A_{1} \sin \left(p x_{3}\right) & \text { Equation } 40 \\
\psi=B_{2} \cos \left(q x_{3}\right) & \text { Equation } 41 \\
u_{1}=\left[i k A_{1} \sin \left(p x_{3}\right)-p B_{2} \sin \left(q x_{3}\right)\right] & \text { Equation } 42 \\
u_{3}=\left[p A_{1} \cos \left(p x_{3}\right)-i k B_{2} \cos q x_{3}\right] & \text { Equation } 43 \\
\sigma_{31}=G\left[2 i k p A_{1} \cos \left(p x_{3}\right)+\left(k^{2}-q^{2}\right) B_{2} \cos \left(q x_{3}\right)\right] & \text { Equation } 44
\end{array}
$$




$$
\begin{gathered}
\sigma_{33}=-\lambda k^{2} A_{1} \sin \left(p x_{3}\right)-\lambda p^{2} A_{1} \sin \left(p x_{3}\right)-2 G p^{2} A_{1} \sin \left(p x_{3}\right) \\
+2 G i k q B_{2} \sin \left(q x_{3}\right)
\end{gathered}
$$

The non-trivial solution to this system of equations yields Equation 46.

$$
\frac{\tan (q h)}{\tan (p h)}=\frac{\left(q^{2}-k^{2}\right)}{4 k^{2} p q}
$$

\subsection{Numerical solution to Lamb wave equations}

When considering only positive roots of the wave number $(k)$, both Equation 39 and Equation 46 can be solved numerically by initially guessing a frequency, a phase velocity (Equation 47 ) and by following steps as outlined in $[64,65]$.

$$
C_{p}=\frac{\omega_{a v g}}{k_{a v g}}
$$

Following these steps yields dispersion curves as shown in Figure 48. Dispersion curves are important for determining the frequency at which a material is to be interrogated. At minimum, two modes will exist simultaneously and those correspond to $A_{o}$ and $S_{o}$ which are to the left of the line as shown in Figure 48. Excitation at higher frequencies yields additional modes $A_{o}, A_{1}, A_{2}, \ldots . A_{n}$ and their associated symmetric modes. The presence of additional modes makes for difficult analysis of the signal. 


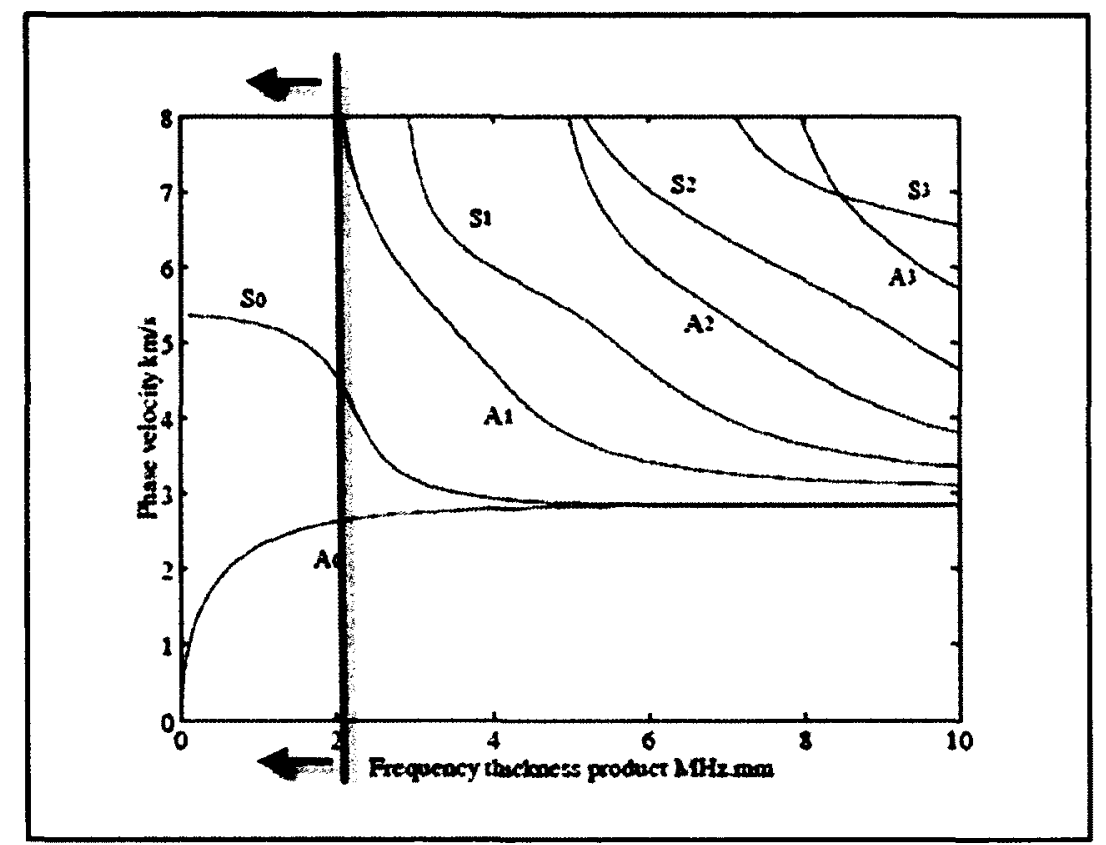

Figure 48: Dispersion Curves for Aluminum [63]

\subsubsection{Analysis of Lamb waves}

Analysis of Lamb waves is very useful for the detection of damage within materials. However, it is very difficult to analyze Lamb waves due to the presence of variables such as: multiple wave modes, dispersion, modal conversion and scattering. Due to all these variables, analysis is very time intensive and is done using Digital Signal Processing (DSP). There are multiple methods used within DSP for carrying out Lamb wave analysis: Time Domain (TD), Frequency Domain (FD), Time Frequency Domain (TFD) and Wavelet Transform Analysis (WTA)[33]. Each of the mentioned methods provides advantages and disadvantages. The simplest method of analysis is the TD analysis and the most difficult is WTA.

Research carried out within this thesis was done using the Acellent system, an AcoustoUltrasonic system that generates Lamb waves to interrogate the health of a structure. The exact algorithm utilized is proprietary, and therefore it is unclear which analysis technique is utilized for damage detection and identification. However, regardless of the techniques used, it is 
known through paper findings in the literature that the Acellent software quantifies damage using a Damage Index (DI) [66]. For that reason, not all analysis techniques have been described in detail, with the exception of TD analysis which is described in section 2.2.3.2.1.

\subsection{Time Domain (TD) analysis}

All transmitted and received signals are captured within the TD; hence, TD analysis is an ideal starting point for data reduction.

Lamb waves, like all other waves, transport energy form one location to the next; presence of damage changes the total energy being transported. TD analysis can therefore be applied to the total energy of the signal for the identification of damage. Analysis of energy can be done using the Hilbert transform $[32,33]$. The Hilbert transform is a method which looks at rapid changes in frequency of the transmitted signal. The change in frequency may be correlated to damage or the presence of other foreign objects. Figure $49(a)$ shows a Lamb wave signal for a damaged aluminum plate while Figure 49 (b) shows the Hilbert transform of the same signal. The Hilbert transform in Figure $49(b)$ indicates a rapid change in frequency of the Lamb wave. This change in frequency may be due to the presence of damage; however, it is hard to determine if this is due to damage or due to other phenomena that may have existed since the manufacturing of the component. To limit the effect of wall reflection and other phenomena that may influence the results, a comparison of the current signal is made to a benchmark signal. 


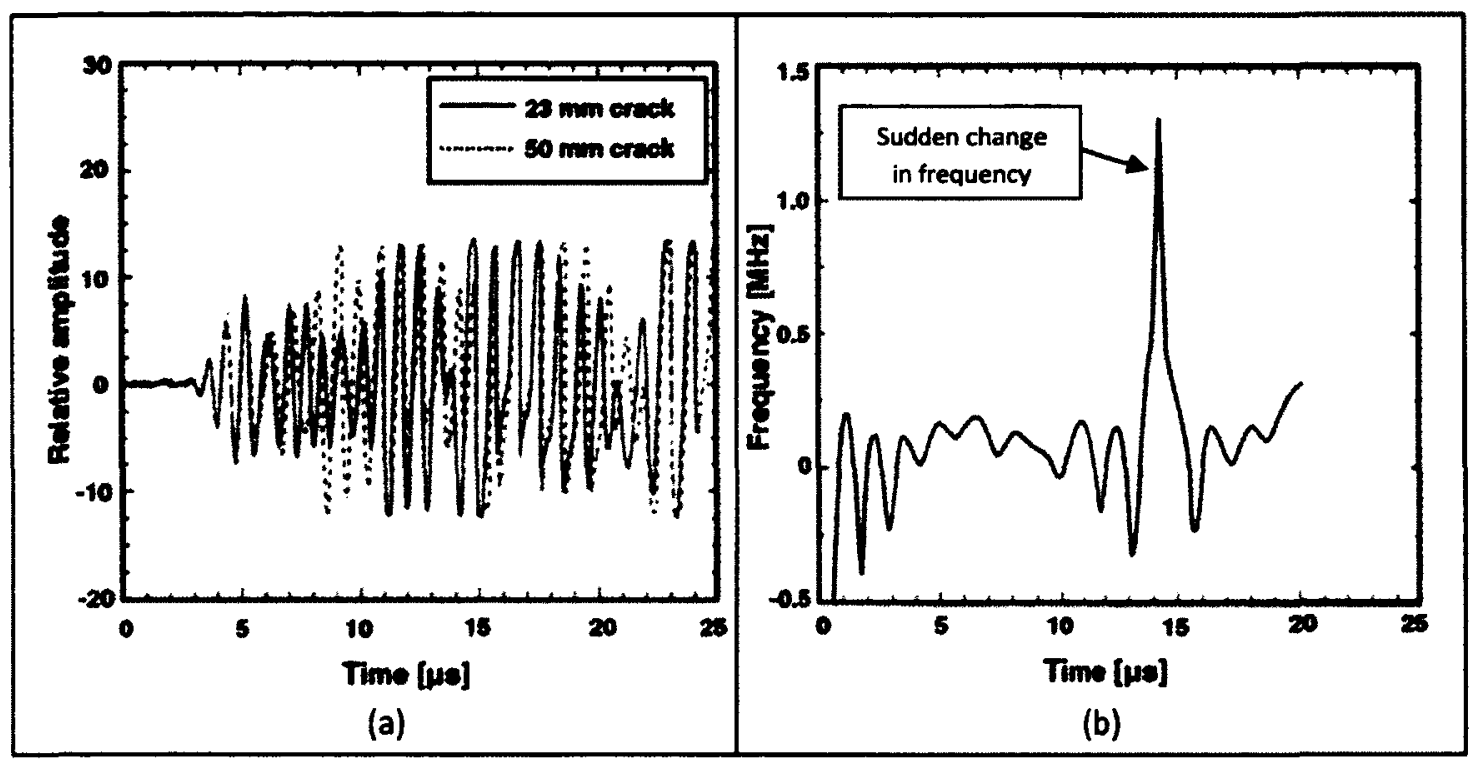

Figure 49: Lamb wave analysis of an aluminum plate: (a) Lamb waves and (b) Hilbert transform [32]

A benchmark for a Lamb wave signal may be obtained by scanning the same component prior to damage formation. A correlation $\left(\lambda_{x y}\right)$ between the benchmark and the damage signal may be identified using Equation 48 [33]. By comparing current signals with benchmark signals, noise which may be a result of reflection off free surfaces may be subtracted as they are also included within the benchmark signal. Figure 50(a) shows gathered benchmark signals for a damage-free composite plate while Figure 50 (b) shows gathered signals for a delaminated composite plate. Figure 50 (c) shows the correlation values for each signal as compared to the inverse signals. Figure 50(d) shows the correlation ratio of the benchmark to the damaged plate. The peak within Figure 50(d) corresponds to the point of greatest difference between the two signals and therefore the point of damage [33].

$$
\lambda_{x y}=\frac{N \sum_{i=1}^{N} x_{i} y_{i}-\sum_{i=1}^{N} x_{i} \sum_{i=1}^{N} y_{i}}{\sqrt{N \sum_{i=1}^{N} x_{i}^{2}-\left(\sum_{i=1}^{N} x_{i}\right)^{2}} \cdot \sqrt{N \sum_{i=1}^{N} y_{i}^{2}-\left(\sum_{i=1}^{N} y_{i}\right)^{2}}}
$$


Where:

- $N$ is the number of samples obtained during data acquisition,

- $x_{i}$ is the amplitude of the benchmark signal, and

- $y_{i}$ is the amplitude of the second signal.

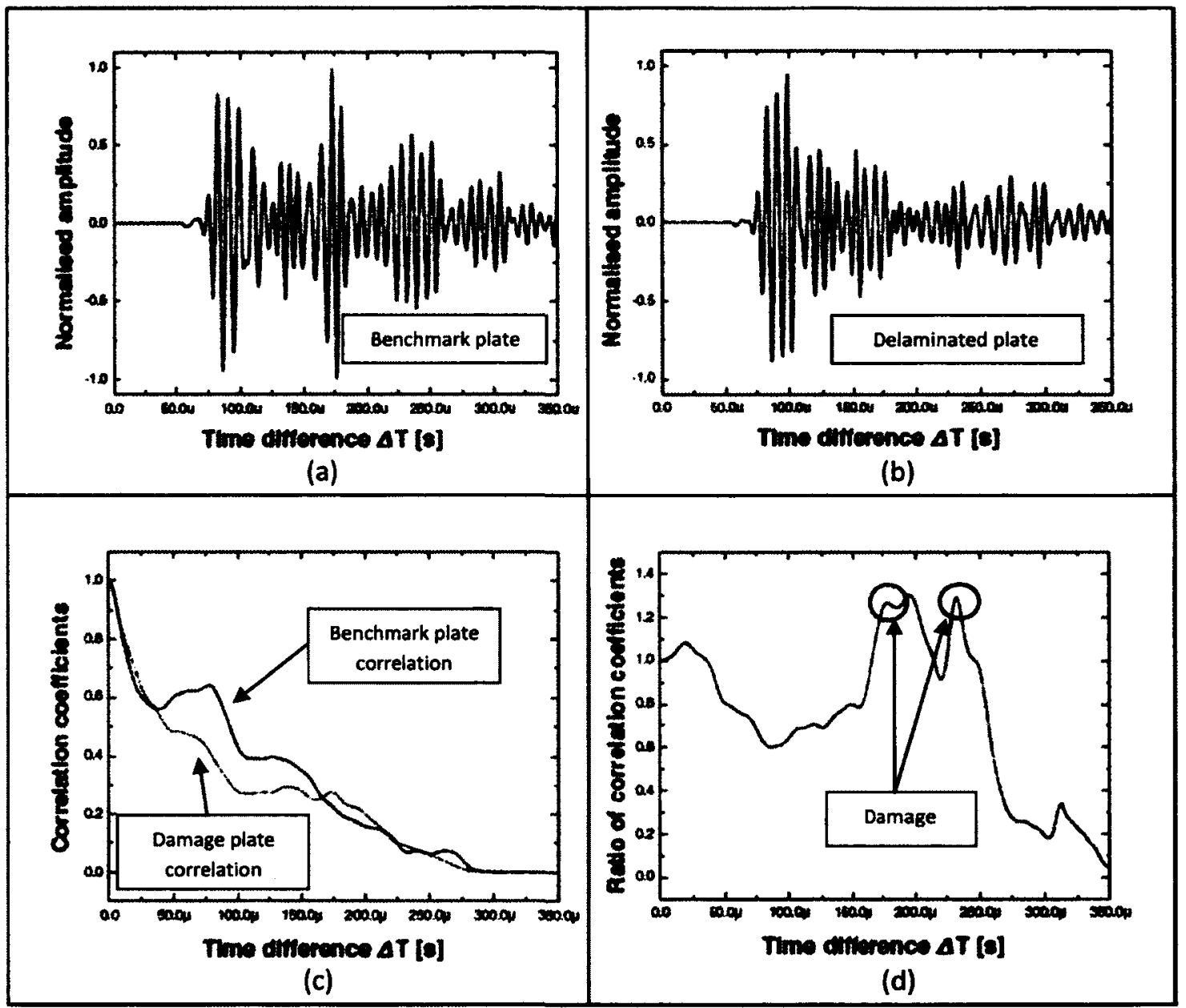

Figure 50: Correlation of benchmark and damaged plate: (a) Lamb waves signal of a benchmark composite plate, (b) Lamb waves of a damaged composite plate, (c) correlation coefficient plot, and (d) the ratio between both correlation coefficients [32]

In addition to the correlation coefficient, DI may be used to determine damage features within a signal. DI is very similar to the correlation coefficient; however, it is only used to examine both amplitude and time delays within either the time or frequency domain. This analysis is very useful for determining the size of damage. Figure 51 shows a typical signal as captured using a sensor. The first received portion of the signal is known as the incident wave; this is almost 
identical to the transmitted signal with the exception of amplitude change. The second portion is the scattered damage wave; this wave is a result of interference between the incident wave and damage. Two signals captured at two different states may be correlated to each other using the DI (Equation 49).

$$
D I=\left(A p_{1}-A p_{2}\right) \times\left(T_{1}-T_{2}\right)
$$

Equation 49

Where:

- $A p$ is the amplitude

- $T$ is the difference of ToF

- The subscripts $1 \& 2$ denote two signal states, one of which may be the benchmark state.

Dis are scalar values which are obtained by comparing two current signals to each other or against a benchmark signal. Assignment of known damage size to the calculated DI correlates damage growth with changes in DI. Therefore, databases within data reduction algorithms may be developed to contain all calculated DI with their associated damage size. Newly calculated DI may be compared to those within the database to interpret damage size. 


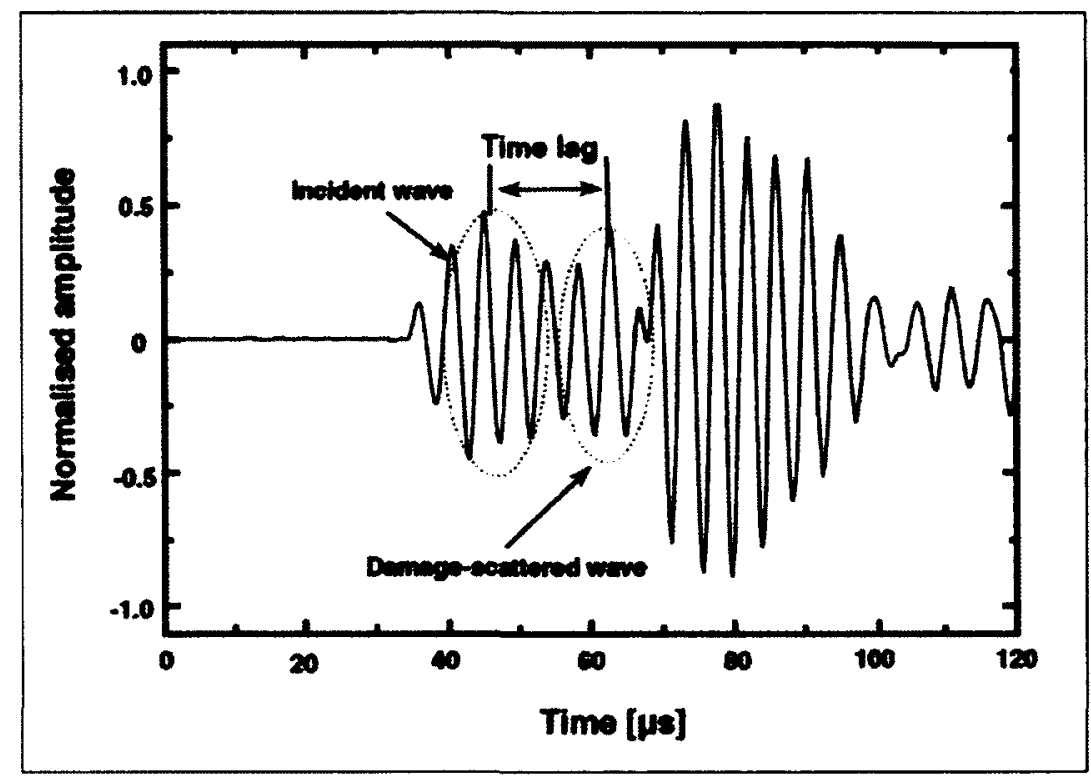

Figure 51: Signal Scatter due to the Presence of Damage [32]

\subsubsection{The Acellent system}

The Acellent system is an Acousto-Ultrasonic system which uses principles of guided waves as those described in section 2.2.3 to detect damage. The system is composed of data acquisition hardware and data analysis/reduction software. The hardware box, also known as ScanGenie•, is used to generate signals and collect them with 32 channels as well as two temperature compensation channels. The ScanGenie box interfaces to a windows-based PC through the use of a USB port. Signal generation parameters as well as data analysis is done using one of three applications that are purchased with the system: SmartMetal ${ }^{\bullet}$, SmartPatch ${ }^{\bullet}$ and SmartComposite ${ }^{-}$Each one of these application is designed to support setup for data acquisition as well as analysis for the detection and quantification of defects. As the system is to be used on the detection of disbond, the SmartPatch application is further described in the following subsections. 


\subsection{SmartPatch ${ }^{\circledR}$ application setup}

The SmartPatch application provides three purposes: system setup, defect detection and defect quantification. Every component being tested using this system requires an independent setup, regardless of similarities. In cases where coupons have identical geometry and sensor placements, setup files will be similar but not identical.

Creation of a new test starts with the setup of sensor locations; this can be done by creating a new sensor layout, or by utilizing a previously used layout as shown in Figure 52(a). Setup of sensors is done using a Cartesian coordinate system. A point on the coupon must be set to the $(0,0)$ position. The exact location of the $(0,0)$ point is not critical, as the purpose of this step is to identify the location of each sensor with respect to the location of all other sensors. These coordinates are used for the determination of signal ToF. Figure $52(b)$ shows the setup of sensors, the number of sensors installed on the component, the number of smart layers, and the unit of measurement which appear in the left hand side of the figure. The analysis of two or more independent components can be done simultaneously by assigning multiple smart layers; this is only done when signals form one layer is known not to affect the other layer. The number of smart layers is always set to the value 1 for all experiments carried out for this research. Figure 53(a) and Figure 53(b) show a single and double smart layer setup, respectively. 


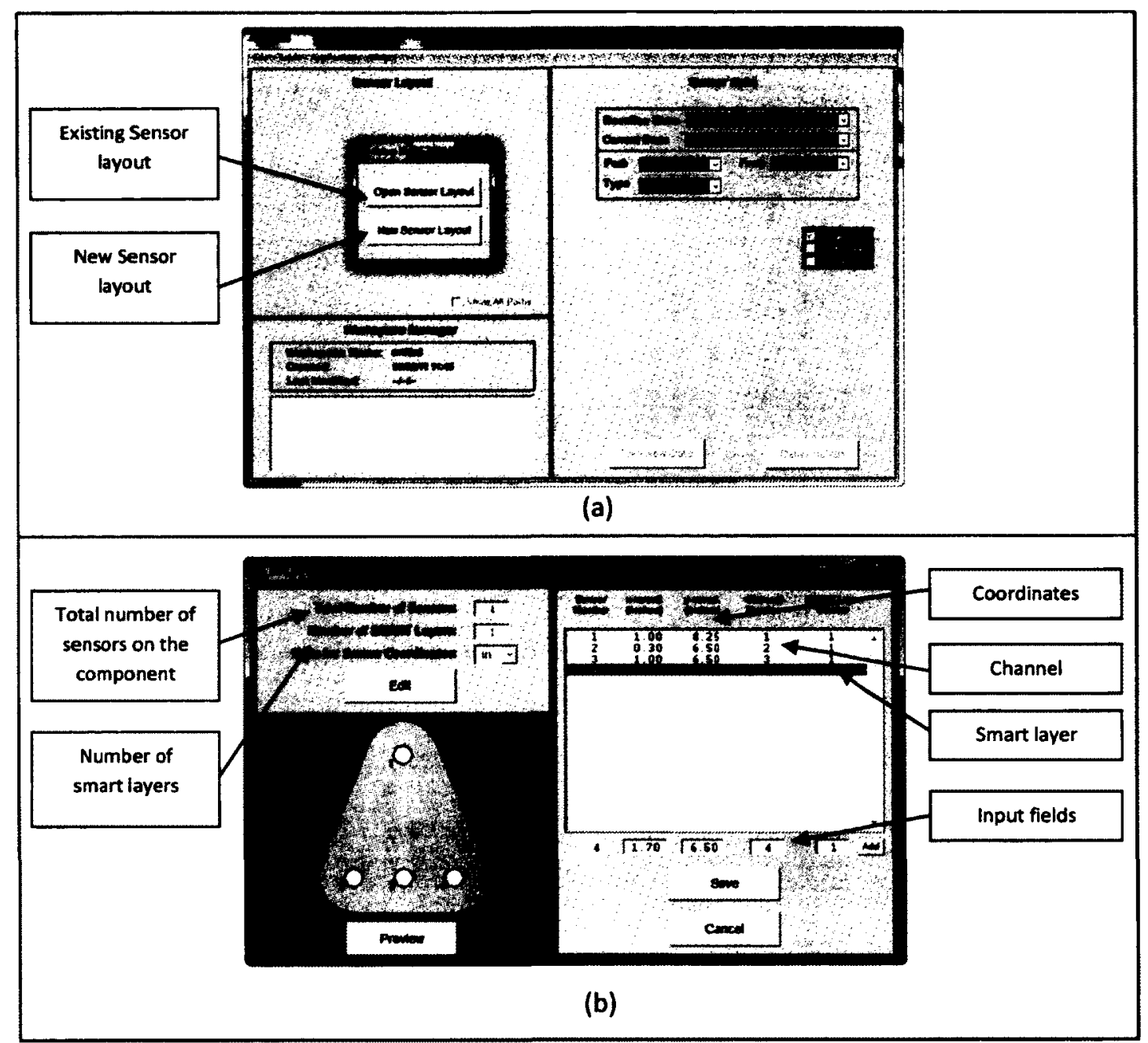

Figure 52: Acellent Sensor Setup: (a) sensor creation and (b) sensor setup

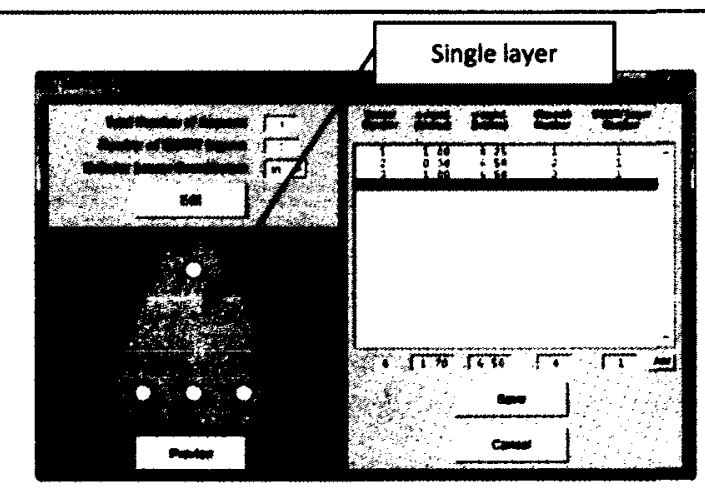

(a)

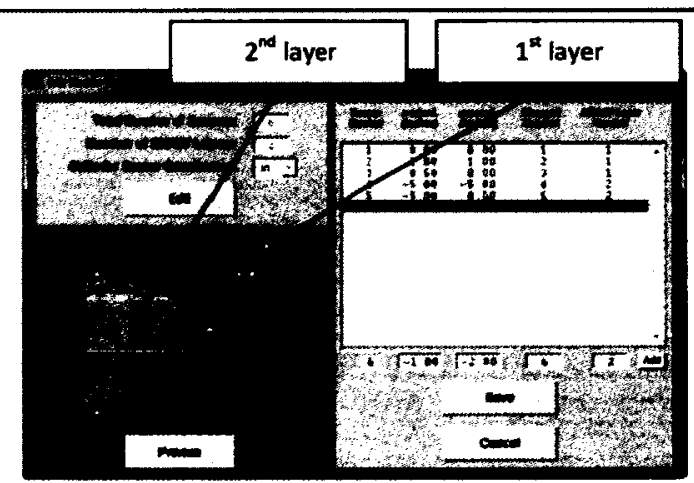

(b)

Figure 53: Smart Layers: (a) single layer and (b) two smart layers 
Following the sensor setup, the Data Acquisition (DAQ) parameters are inputted within the application. The sampling rate identifies the number of samples that are collected per second after the signal has been transmitted. The sampling points identify the total number of samples that are collected and stored. The average number identifies whether multiple samples may be averaged and recorded as a single value within the application. All three parameters are setup within the window which is shown in Figure 54.

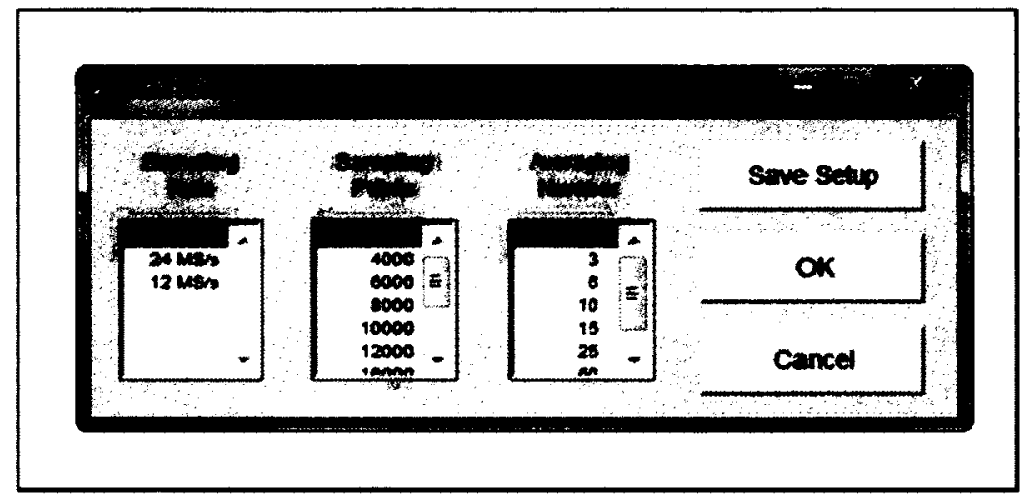

Figure 54: DAQ Setup

The final setup step is the path definition step; this step defines which sensors will transmit signals and which sensors will receive signals. Paths defined within the application are done to determine scanning areas for damage detection. This setup differs for each component; as signals were highly dependent on the piezoelectric element configuration and signal propagation, which are influenced by factors such as adhesion thickness underneath the sensor. Paths can be created within the path definition manager as shown in Figure 55(a). Each path as defined using the window in Figure 55(b) has an actuator sensor, receiver sensor(s), number of signal bursts, voltage inputs and signal gain. The actuator sensor denotes the sensor which acts as a transmitter, while the receiver sensor(s) will be those that act as signal receptors. The signal type refers to the number of pulses that are transmitted within each signal. Voltage defines the input voltage to the sensor and signal gain defines the gain setting for the received signal. Figure 
55(c) shows the window for which frequencies may be set for path definitions. In addition to selecting a single scanning frequency, multiple frequencies may be used. The system is capable of scanning the same path utilizing multiple frequencies which may be used for analysis. An initial and final frequency is selected and a stepping interval is defined to create all the necessary parameters for that path. Multiple frequencies are scanned as the optimal frequency to generate only two modes may be unknown.

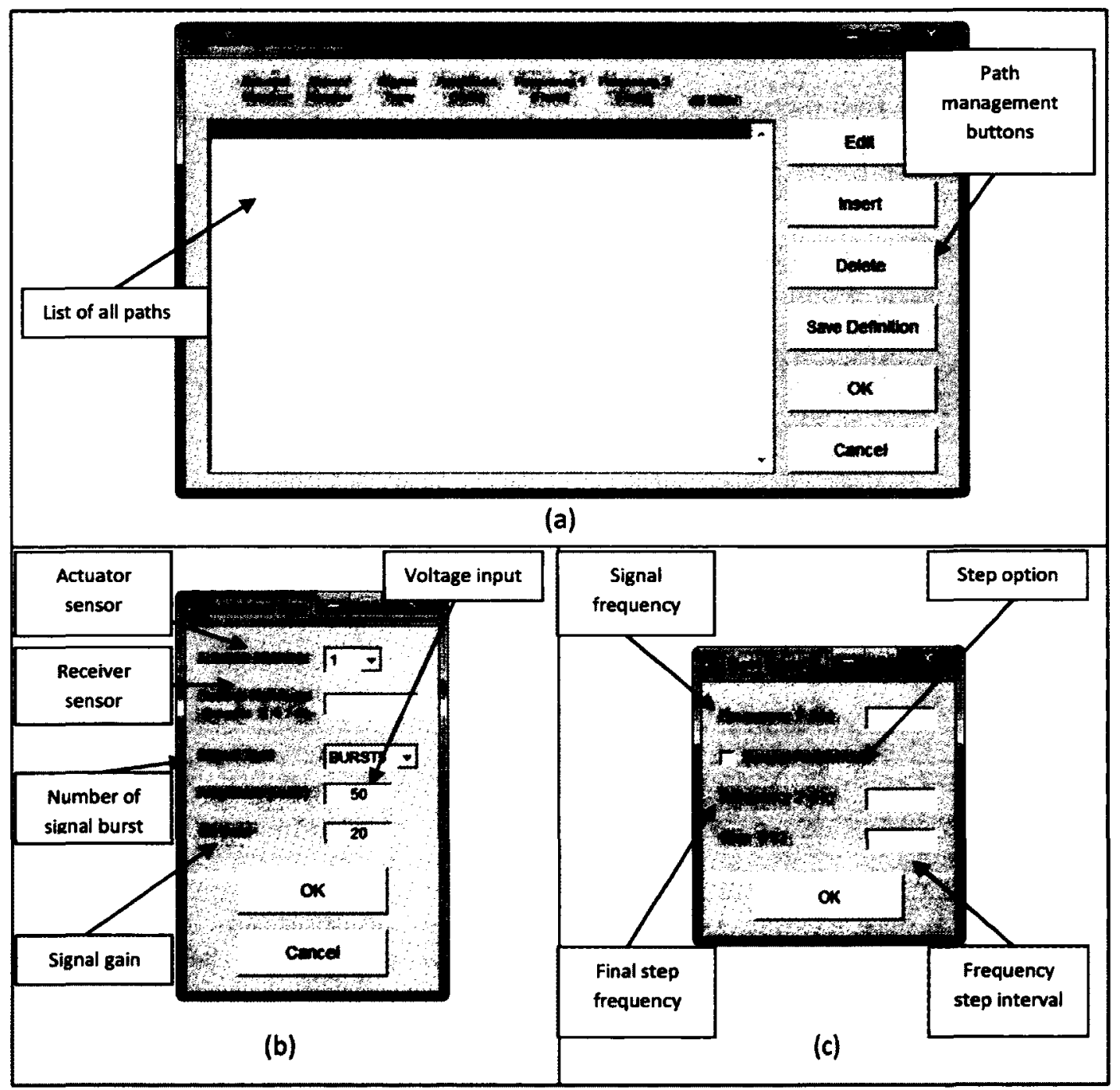

Figure 55: Path Definition: (a) path definition management, (b) path initiation and properties and (c) path frequencies 
After path definition, a benchmark reading is acquired, similar to that shown in Figure 56(a). Gains within the path definition were individually adjusted so that the bursts $6-10$ are between $0.5 \mathrm{~V}$ and $1.0 \mathrm{~V}$ as shown in Figure $56(\mathrm{~b})$. Setting gains so that voltage is between $0.5 \mathrm{~V}$ and $1.0 \mathrm{~V}$ ensures that energy drops may be correlated to a specific damage size. The system is now ready for data acquisition and new readings are taken using the "take new data" button as shown in Figure 57.

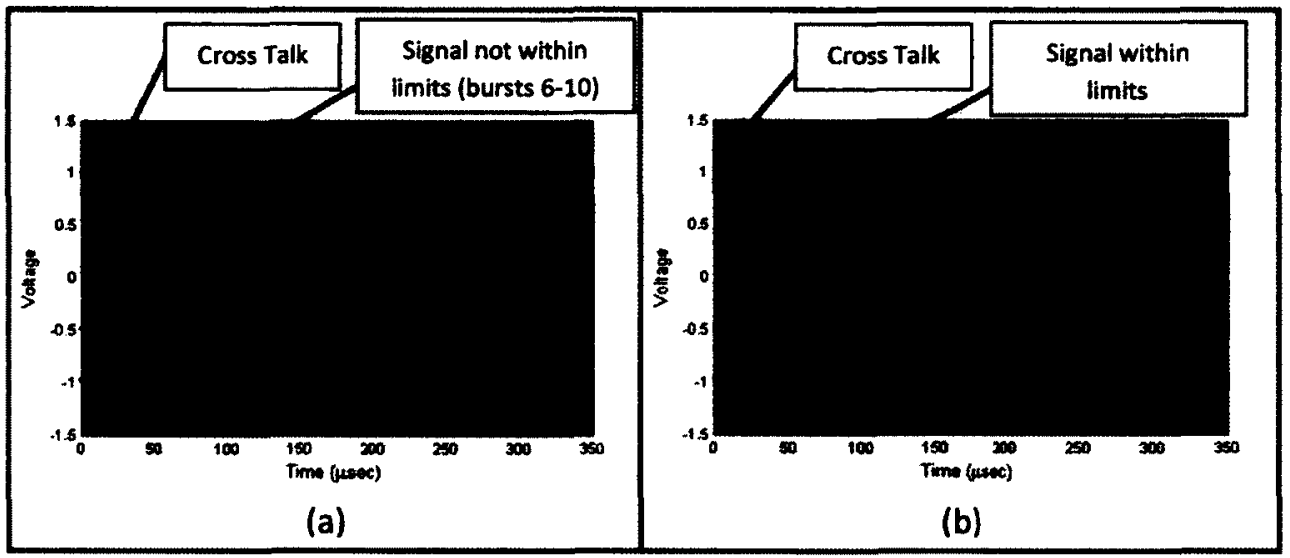

Figure 56: Signal Propagation Settings

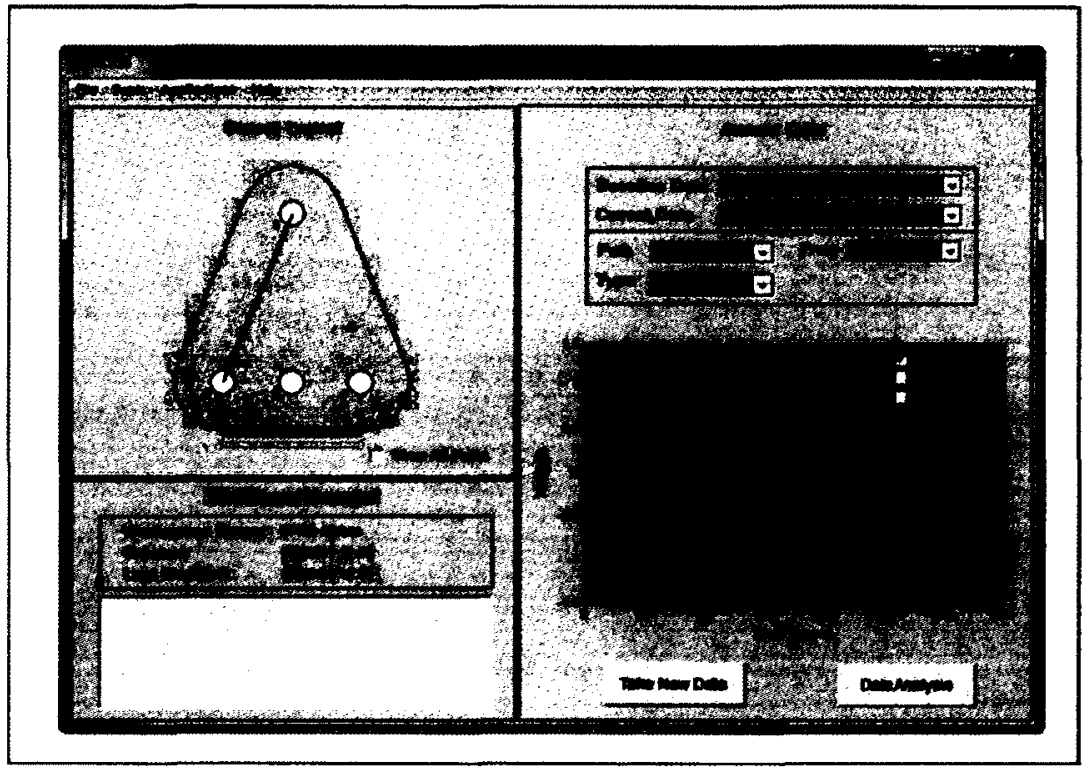

Figure 57: SmartPatch ${ }^{\bullet}$ Main Window 


\subsection{The Acellent system analysis}

Signals acquired using the SmartPatch application are analyzed using two built-in utilities, one for the detection of damage and the second for the quantification. Detection of damage is done using the Direct Path Image (DPI) utility, while the quantification of damage is done using the Semi-Empirical Damage Sizing (SEDS) utility. Figure 58 shows an example of a coupon being analyzed using the DPI. Within the figure, a digital representation of the coupon is shown with all installed sensors. Paths which were scanned during the data acquisition step are analyzed within the software to produce images for defect identification. Damage is indicated on the digital representation images as shown in Figure 58. The intensity of damage may be evaluated using the threshold value. The threshold correlates signal amplitude (i.e. energy) changes to the application's color scale. Adjusting the threshold to a high value will only display changes in signals that are very large while a low threshold will display almost all signal amplitude changes. This is the reason for which the benchmark reading was setup so that bursts $6-10$ are between $0.5 \mathrm{~V}$ and $1.0 \mathrm{~V}$. Additional analysis may be carried out on different portions of the signal by adjusting the time window shown in Figure 58. However, for all analysis within this research, the signal is truncated to only contain burst $6-10$, as signal reflection from component edges should not be included within the analysis. 


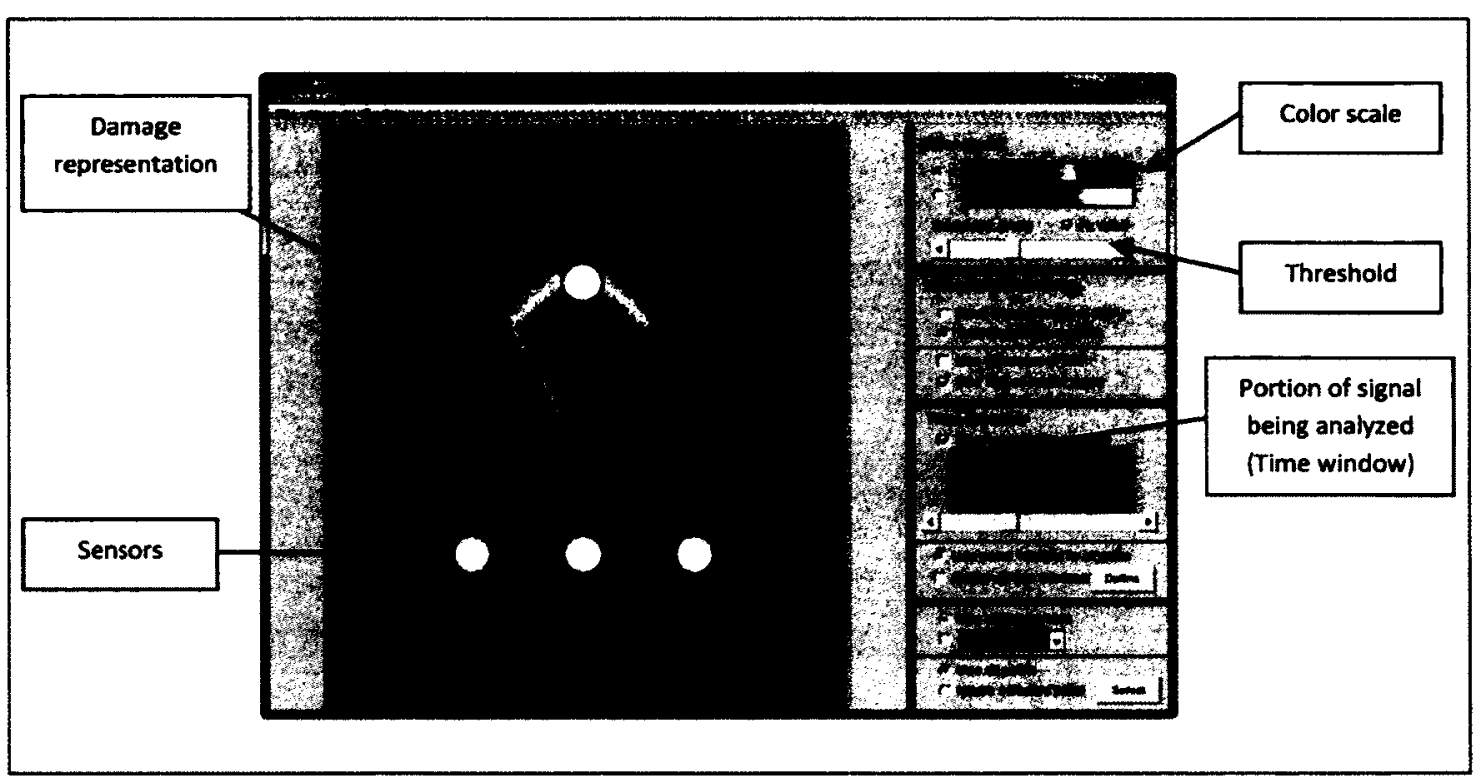

Figure 58: Direct Path Image Analysis

The SEDS utility uses all collected signal data with corresponding life cycles and some measured damage sizes to interpret damage size of unknown points as shown in Figure 59. Data input within this step is used to compute the DI and uses them to output interpretations of damage sizes as those shown in Figure 60. 


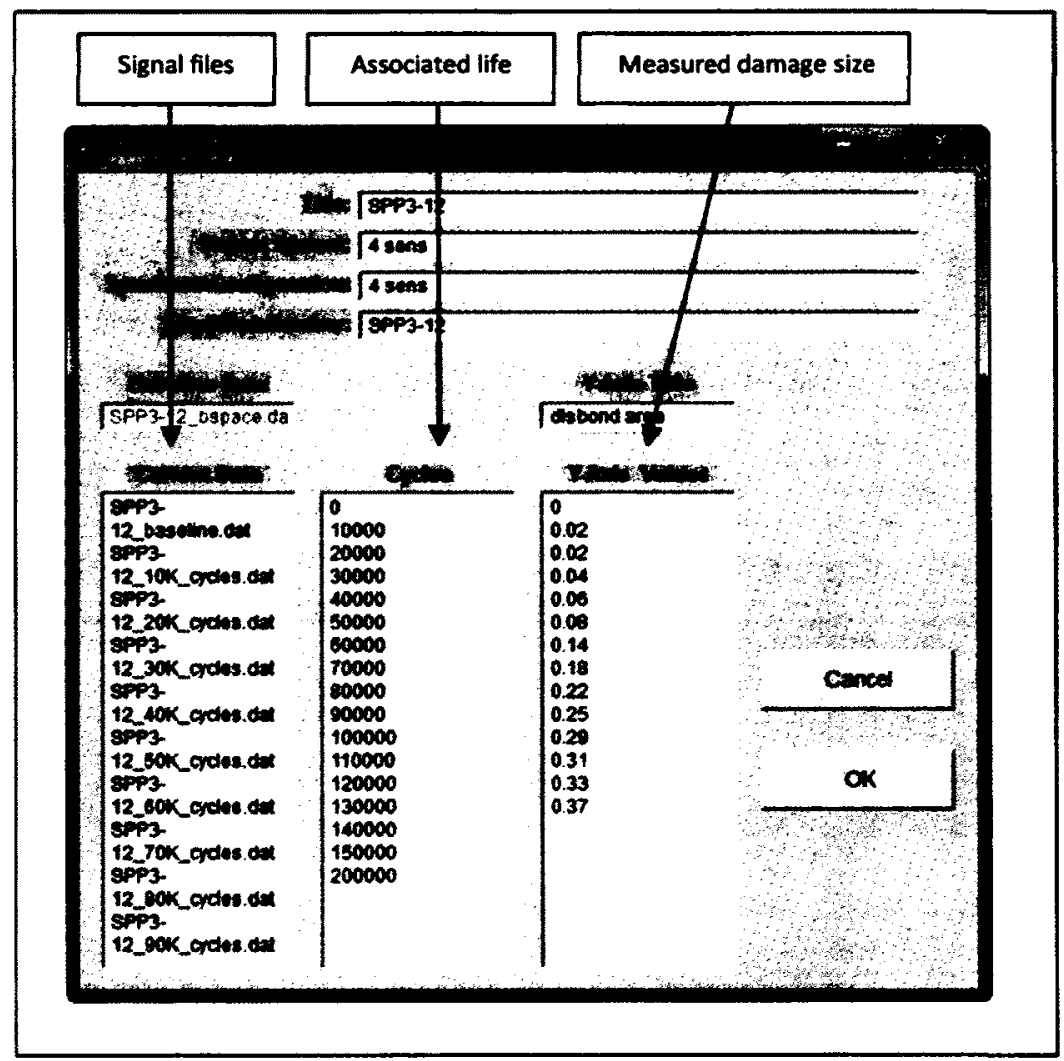

Figure 59: Semi-Empirical Input

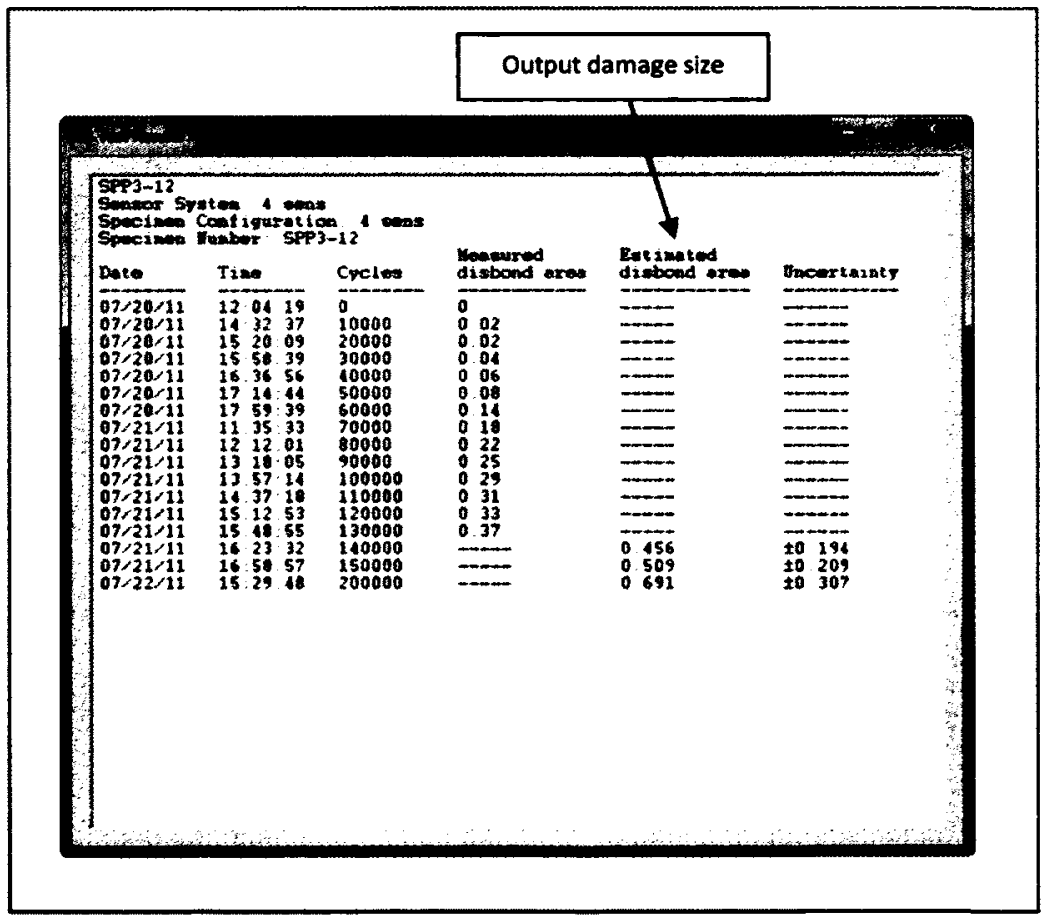

Figure 60: Semi-Empirical Output 


\section{Chapter 3.0: Test Design and Coupon Manufacturing}

Structural Health Monitoring (SHM) has been researched for many years. There are many SHM systems available; however, some are more effective for detection of certain damage modes as compared to others. The objective of this thesis was to test readily available SHM solutions for the detection and quantification of crack growth as well as disbond. Testing the SHM system for the detection of both crack growth and disbond required the development of different coupons as well as test plans.

Three different types of coupons were developed to test the effectiveness of three SHM systems for the detection and quantification of crack growth as well as disbond. The first type of coupons was of metallic construction with a center surface crack; they were designed so that the SHM systems may be used for the detection and quantification of crack growth. The second type of coupons was of composite patches bonded to a metallic substrate design; they were designed so that the SHM systems may be evaluated for the detection and quantification of both crack and disbond growth. The third type of coupon consisted of composite patches bonded to a composite substrate design; they were designed so that the SHM systems may be evaluated for the detection and quantification of disbond growth.

This chapter is divided into three subsections: coupon design, coupon manufacturing and coupon instrumentation.

\subsection{Coupons}

All three sets of coupons were manufactured using different processes as each set was composed of different materials and had different requirements. Metallic and composite-to-metallic coupons were designed and manufactured during earlier stages of the Smart Patch 
Project (prior to the start of this thesis project) $[29,39]$ and therefore in-depth design and manufacturing processes are not included within this chapter.

\subsubsection{Metallic Coupons (Coupon Set 1)}

Metallic coupons measuring $15.0 \times 2.0 \times 0.25$ inches were manufactured of 2024-T3 aluminum. These coupons were designed to determine the effectiveness of SHM systems for the detection and quantification of cracks within metallic components. The dimensions for these coupons were chosen so that internal stresses of the coupon did not exceed their yield strength (40 KSI); this correlates to a static load of $20,000 \mathrm{lb}$.

All metallic coupons were initially machined with a rib along the length of the coupon as shown in Figure 61. An Electronic Discharge Machining (EDM) notch was created at the surface of the rib so that crack nucleation and propagation is allowed during fatigue of the coupons. Coupons were fatigued until cracks for all coupons grew through the rib and into the aluminum substrate as shown in Figure 62 . Cracks were allowed to grow until surface cracks were approximately 0.20 inches in length as shown in Figure 62(a). Ribs were machined and the cracks were left exposed as shown in Figure 62(b). To grow cracks, coupons were fatigued at maximum load of $13,000 \mathrm{lb}(26 \mathrm{KSI})$ and minimum load of $1,300 \mathrm{lb}$ (2.6 KSI) [39]. Surface Mountable Crack Sensors (SMCS) were employed around the rib as shown in Figure $62(c)$ so that upon crack growth through the rib and into the substrate, the SMCS would trip and fatigue cycles would stop. This was done to limit surface crack lengths to approximately 0.25 inches. To obtain the final measurement of crack lengths, ultrasound, eddy current and a travelling microscope were utilized [39]. 


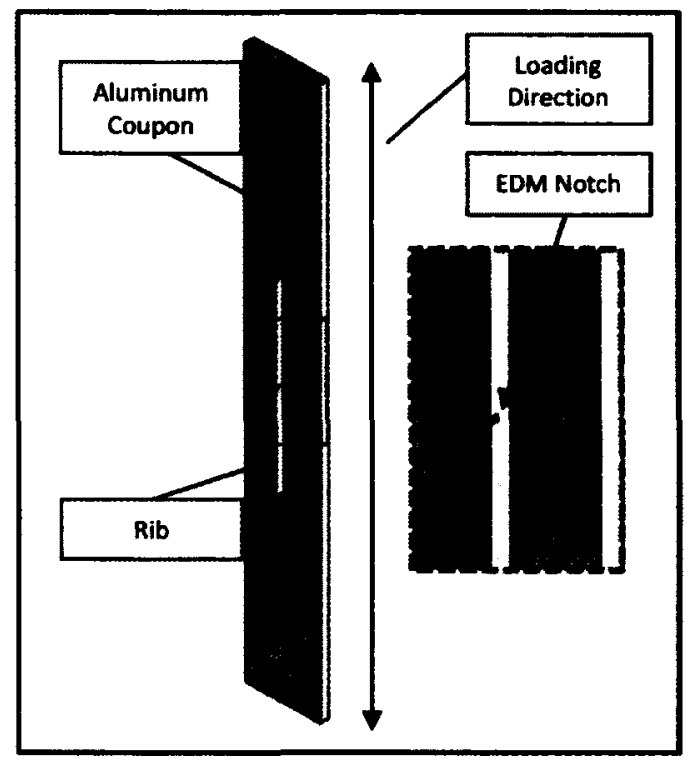

Figure 61: Aluminum Coupon with EDM Notch

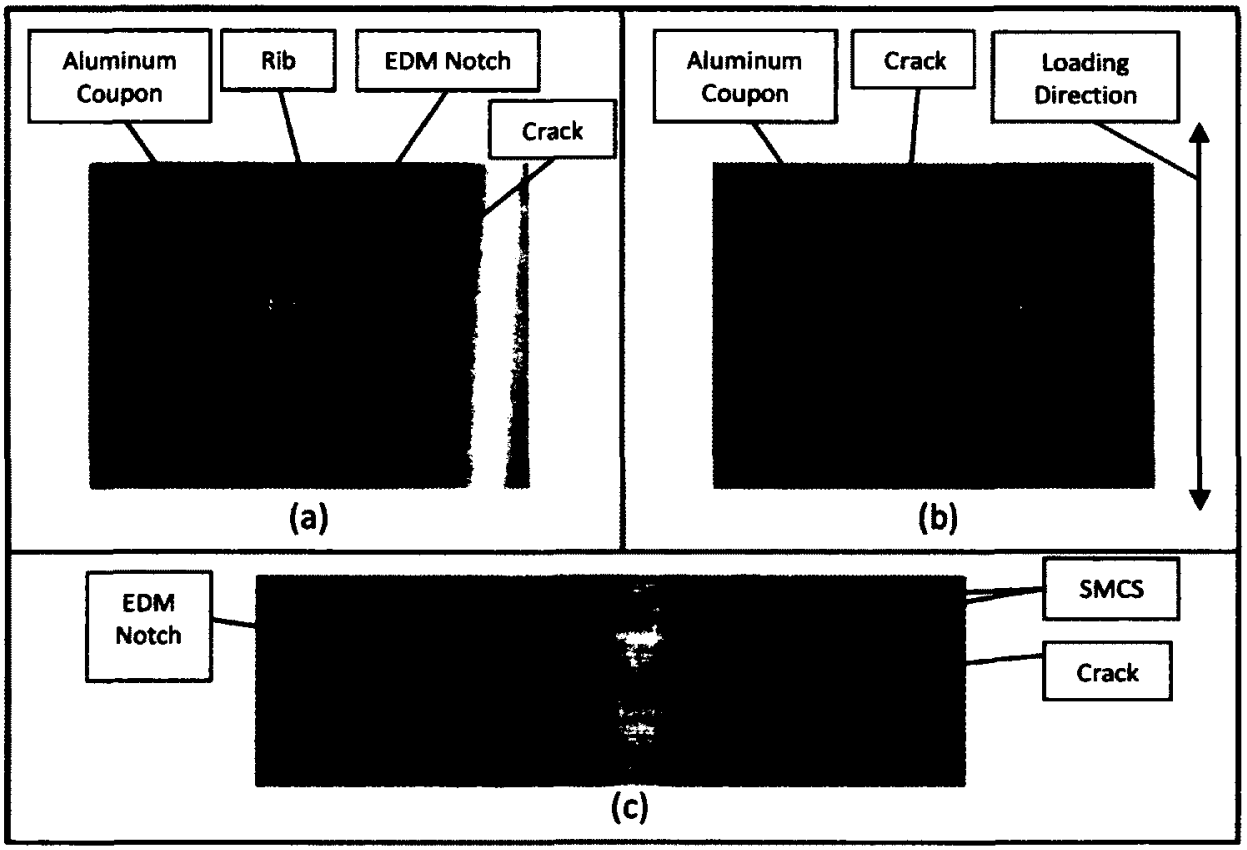

Figure 62: Aluminum Coupon Crack Growth: (a) Fatigued coupon with rib, (b) Fatigued coupon removed rib and (c) SMCS instrumented coupon prior to rib removal 


\subsubsection{Patched Metallic Coupons (Coupon Set 2)}

Metallic coupons measuring $15.0 \times 2.0 \times 0.25$ inches manufactured of 2024-T3 aluminum and carbon fibre patches (doublers) measuring $5.0 \times 2.0$ inches were manufactured and are shown in Figure 63(a) and Figure 63(b). In addition to the patch shown in Figure 63(b), a rectangular patch measuring $5.0 \times 2.0$ inches was also manufactured. A rectangular patch and the patch shown in Figure 63(b) were bonded on either side of the substrate using FM73 film adhesive (an aerospace grade film adhesive later described in section 3.1.3.1); this was done to ensure no unsymmetrical bending took place during loading of the coupon. The patches were manufactured using carbon/epoxy AS4-3501 carbon fibre prepreg tape with a layup of $[0,90,0]_{s}$ [29]. These coupons were designed to determine the effectiveness of SHM systems for the detection and quantification of disbond. In addition to the detection of disbond, some coupons were manufactured with a center surface crack within the substrate; this was done so that simultaneous detection and quantification of both cracks and disbond growth may be investigated [39]. Exact dimensions of these coupons were determined using Finite Element Analysis (FEA) [29]. FEA was used to determine the size, shape and layup of the patch so that disbond would occur during static loading of the coupons. In addition to static loads, the FEA took into account thermal loads so that thermal residual stresses may be computed. Thermal residual stresses are stresses that are introduced during the bonding and curing cycle of the FM73 adhesive [29]. Curing of the FM73 film adhesive required the application of high temperature and pressure. In the presence of high temperatures, the aluminum substrate expanded while the carbon fibre contracted. Expansion of the aluminum and contraction of the carbon fibre was due to a positive thermal expansion coefficient and negative thermal expansion coefficient, respectively [67]. As temperature decreases, the aluminum begins to 
contract and the carbon fibre begins to expand, however both are adhered together. This process introduces stresses within the coupon.

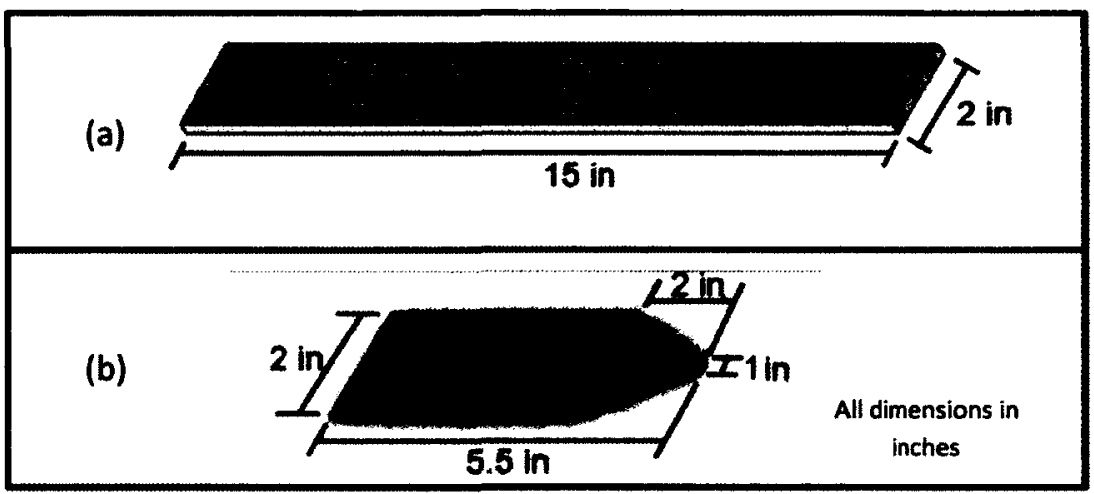

Figure 63: Smart Patch Composite-Metallic Coupon Design: (a) Substrate and (b) Patch

\subsubsection{Composite-to-Composite Coupons (Coupon Set 3)}

Composite-to-composite coupons were designed and manufactured so that the effectiveness of the SHM systems may be evaluated for the detection and quantification of disbond between two adhesively joined composite materials. Unlike metallic coupons, composite coupons are non-isotropic and therefore SHM systems' effectiveness will be different as wave propagation, thermal and electrical properties are different. An FEA analysis was carried out using ABAQUS to determine the effects of static and thermal loads for the purpose of inducing disbond. This was done on multiple proposed coupon designs.

This section is divided into three subsections, the first section discusses design of the coupon, the second discusses the manufacturing of the coupons and the third discusses the instrumentation of the coupons using three different SHM systems. 


\subsubsection{Coupon design requirements}

In addition to other evaluation requirements, SHM systems used within this study were tested for their effectiveness for the detection of disbond between adhesive joints on composite components. For this reason, the coupons were specially designed with a joint that would fail at a predefined location with high repeatability. This approach was done to limit the amount of NDE inspections required to determine and locate failure within the adhesive; this allowed for quick SHM system evaluation. To meet the overall objective, a series of requirements were composed to aid in the design of the coupons; these requirements are listed in Table 1.

Table 1: Coupon Design Requirements

\begin{tabular}{|c|c|c|}
\hline $\begin{array}{l}\text { Requirement } \\
\text { Number }\end{array}$ & Requirement Name & Requirement Text \\
\hline 1. & Coupon & The designed coupon must contain an adhesive joint that must fail during testing. \\
\hline 2. & Material Utilization & The designed coupon must be composed of two distinct materials. \\
\hline 2.1. & Materials Composition & Both materials used within this study must be composite. \\
\hline 2.2. & Substrate & The substrate must be composed of carbon fibre material. \\
\hline 2.3. & Patch & The patch must be constructed of a different composite material. \\
\hline 2.4. & Adhesive & The adhesive must be of consistent thickness. \\
\hline 3. & Dimensions & $\begin{array}{l}\text { The coupon must be designed to fit within a test frame with a maximum grip width } \\
\text { of } 4 \text { inches. }\end{array}$ \\
\hline 3.1. & Length & The coupon must be at least 15 inches in length. \\
\hline 3.2. & Width & The coupon must be at least 2 inches in width. \\
\hline 4. & Loading & $\begin{array}{l}\text { The coupon must be able to withstand a load without failure other than that of } \\
\text { disbond. }\end{array}$ \\
\hline 4.1. & Disbond Loading & The patch must disbond from the substrate at a load less than $20,000 \mathrm{lb}$. \\
\hline
\end{tabular}

To meet design requirements, three materials were chosen. Materials of choice were based on availability, cost and mechanical properties. The carbon fibre used for this project was the CYCOM 5276-1 G40-800 unidirectional prepreg tape. This material was chosen as it is used 
widely throughout multiple projects at National Research Council Canada (NRC) and therefore was readily available. CYCOM 5276-1 G40-800 prepreg tape material has a modulus of $22.5 \mathrm{Msi}$, which will vary depending on layup of the manufactured laminates. Specialty Materials $5521 \mathrm{~F}$ unidirectional tape (a boron fibre prepreg) was selected as the material forming the patch that was bonded to the carbon fibre component. Unlike carbon fibres, boron fibres have a modulus of 27 Msi. Differences between moduli allowed for a large enough difference in stiffness and therefore planar stress along the surface of the substrate would be higher than the shear stress of the adhesive, promoting disbond while statically loaded. Selection of another carbon fibre for the patch would result in similar moduli and therefore disbond may or may not have initiated. In addition to its modulus, boron fibre composites were used as repair patch materials for repairs on aircraft requiring a thermal coefficient that is similar to that of metallic components.

Joining of the carbon fibre and the boron fibre cured components was achieved through the use of FM73 toughened epoxy film adhesive. FM73 film adhesive is a widely used adhesive within the aerospace industry as it has excellent mechanical properties for operational temperatures of aircraft. FM73 also has an imbedded mesh that provides thickness control of the adhesive layer while temperatures and pressure are applied during the curing process.

In addition to possessing a required combination of mechanical properties, all selected materials were available at the NRC as they have been used within other ongoing projects.

\subsubsection{Material properties}

Three materials were used for the for the development of the FEA model: CYCOM 5276-1 G40800 Tape, Specialty Materials 5521F Tape and CYTEC FM 73 toughened epoxy film as described in section 3.1.3.1. Property values for all materials were obtained from manufacturer's data 
sheets and are summarized within Table 2. However, there existed some material properties that were not provided; these properties are indicated by an asterisk within the table. Values used for these properties were based on other publications [67].

Table 2: Material Properties

\begin{tabular}{|c|c|c|c|}
\hline Property & $\begin{array}{c}\text { CYCOM 5276-1 G40-800 } \\
\text { Tape [68] }\end{array}$ & $\begin{array}{c}\text { Specialty Materials 5521F } \\
\text { Tape [69] }\end{array}$ & $\begin{array}{l}\text { FM } 73 \text { toughened epoxy } \\
\text { film [70] }\end{array}$ \\
\hline EIRTA & $22.5 \mathrm{Msi}$ & $27 \mathrm{Msi}$ & $300 \mathrm{Ksi}$ \\
\hline E2 RTA & $2.5 \mathrm{Msi}$ & $2.7 \mathrm{Msi}$ & $300 \mathrm{Ksi}$ \\
\hline E3RTA & $2.5 \mathrm{Msi}$ & - & $300 \mathrm{Ksi}$ \\
\hline G12 RTA & $0.7 \mathrm{Msi}$ & $0.84 \mathrm{Msi}$ & - \\
\hline G12 RTA & $0.07 \mathrm{Msi}$ & $0.84 \mathrm{Msi}$ & - \\
\hline G13 RTA & $0.07 \mathrm{Msi}$ & $0.84 \mathrm{Msi}$ & - \\
\hline N12 & $0.3^{*}$ & $0.3^{n}$ & - \\
\hline N12 & $0.3^{*}$ & $0.3^{*}$ & - \\
\hline N12 & $0.3^{*}$ & $0.3^{*}$ & - \\
\hline $\mathbf{v}$ & - & - & 0.3 \\
\hline a11 & $-9.5 \times 10$ & $2.5 \times 10$ & $2.8 \times 10$ \\
\hline $\mathbf{a 2 2}$ & $1.45 \times 10^{-5}$ * & $2.5 \times 10$ & $2.8 \times 10$ \\
\hline a33 & $1.45 \times 10^{-5}$ * & $2.5 \times 10$ & $2.8 \times 10$ \\
\hline
\end{tabular}

\subsubsection{Finite Element Analysis (FEA)}

A Finite Element Analysis (FEA) study was carried out to determine an optimal coupon design that would cause high shear or peeling stresses in the presence of an applied load. These induced stresses caused the boron fibre patch to disbond from the carbon fibre substrate. For all these models, only the planar shear stress was considered for the analysis. Peeling stresses were low according to published values, and therefore designing adhesive failure due to shearing would have exceeded the values required for peeling.

Three models were constructed for the design of this coupon within ABAQUS $\bullet 6.8-1$. The first model utilized ABAQUS ${ }^{\bullet}$ standard shell elements (S4R) which are 4-node elements and $3 D$ stress elements (C3D8R) which are 8-node linear elastic brick elements. The second model utilized standard continuum shell elements (SC8R) which are 8-node quadrilateral in-plane elements and 3D stress elements (C3D8R) which are 8-node linear bricks. The third model utilized 3D 
stress element (C3D20) which is a 20-node quadratic reduced integration bricks and 3D stress elements (C3D8R) which are 8-node linear bricks, reduced integration. S4R, SC8R and C3D20 elements were used on composite components within each one of their respective models while C3D8R elements were used only on the adhesive for each of the models. Due to the computational properties of elements used within each model, each of the models was used for specific application(s). Conventional shell elements allow for computation of planar stresses and are not adequate for the analysis of out-of-plane stresses. Also, models utilizing this type of element were assigned a single element through the thickness of the meshed component. For these reasons, the first model was used with its inherent approximations to quickly evaluate parameters such as geometry and composite layup that would be required to induce disbond within the adhesive joint. Continuum shell elements used within the second model are similar to conventional shell elements with the exception of through-thickness properties. Through the use of continuum shell elements, multi-layer composites were modeled to evaluate planar stresses on each composite ply; this was achieved by assigning a layer of shell elements to each composite ply. Results of model 2 were compared to those of model 1 . The third model utilized 3D stress elements. These elements allowed for the computation of stress and strains in all directions. Thermal residual stresses which were induced during the bonding process could not be computed through the use of the previous two models as elements used within those models allowed for the calculation of only planar stresses while thermal expansion occurs in all three axes. Through the use of 3D stress elements, model 3 was used to compute thermal residual 3D stresses.

A mesh dependency analysis was carried out for each of the three models to determine the minimum amount of elements required to have converged stresses. Multiple analyses were carried out on each model, each with increasing element count. Stresses for a common node 
found in each analysis were allowed to converge to $10 \%$ of the previous two analyses. Figure 64 shows the location of the common node used for each analysis. Figure 65 shows stresses for model 1 versus the number of elements; each point on this graph corresponds to a different analysis. It is evident from the graph, that model 1 converges using approximately 50,000 elements. Figure 66 shows stresses for model 2 versus the number of elements. It is evident from the graph that model 2 converges using approximately 260,000 elements. Figure 67 shows stresses for model 3 versus the number of elements. Model 3 was found to converge using 170,000 elements.

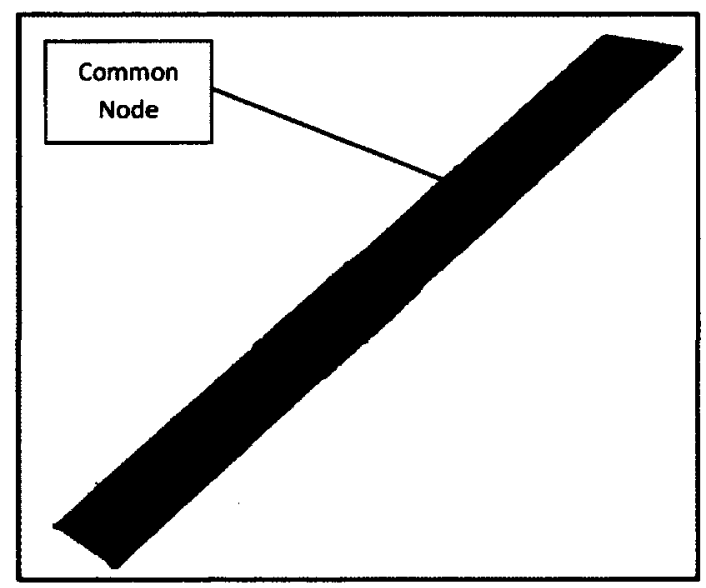

Figure 64: Common Stress Node 


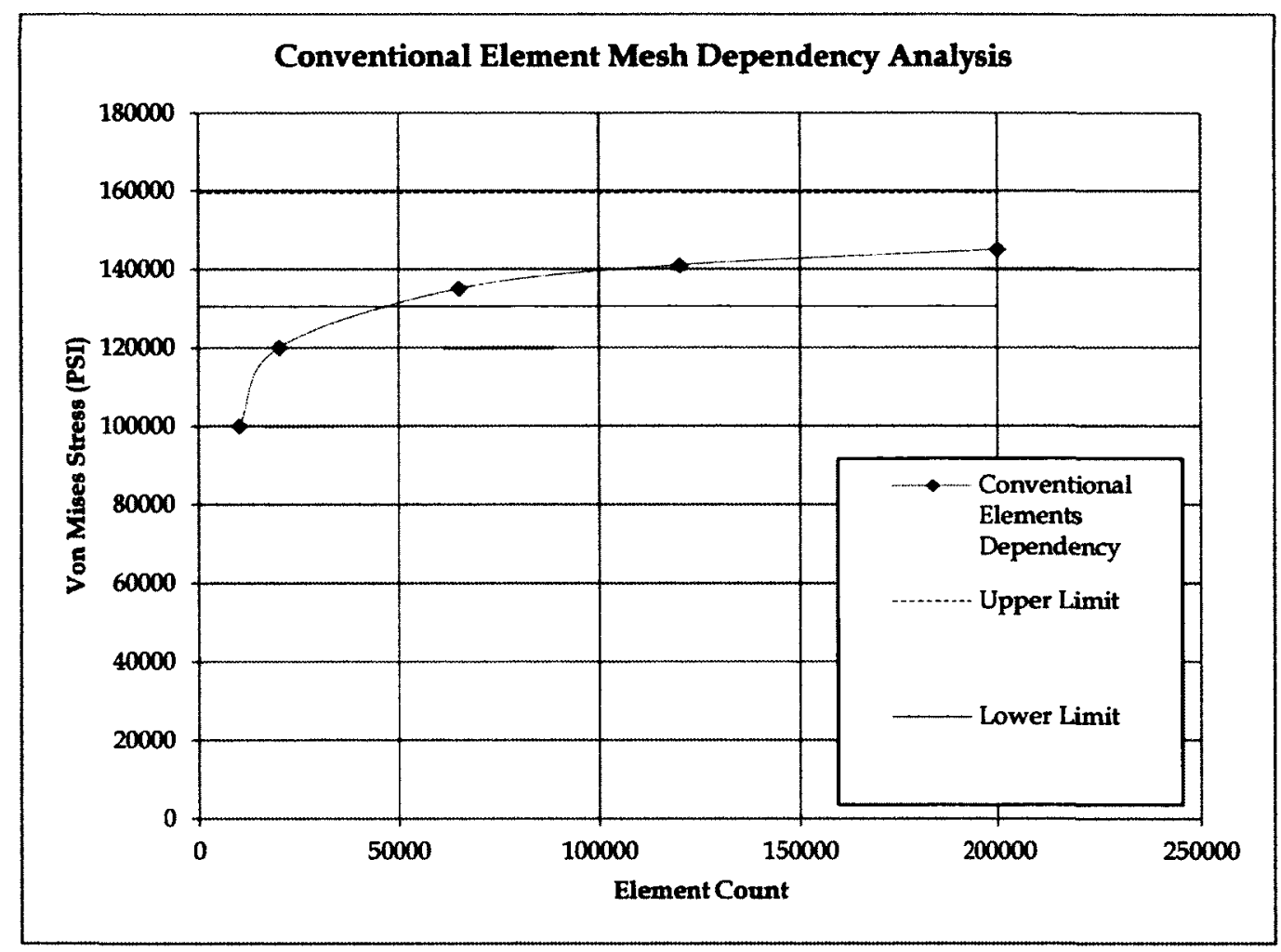

Figure 65: Conventional Shell Element Mesh Dependence Analysis 


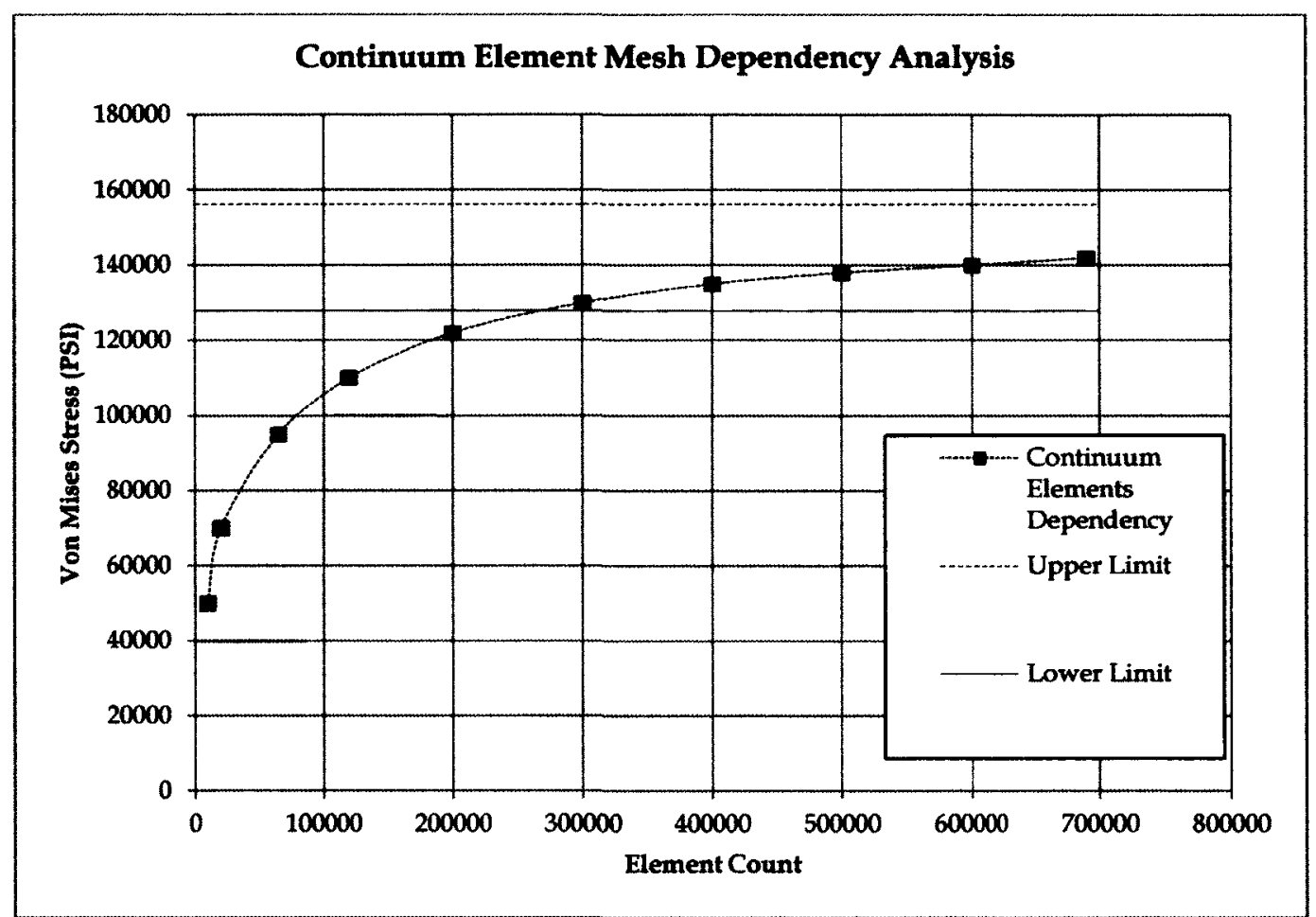

Figure 66: Continuum Shell Element Mesh Dependence Analysis

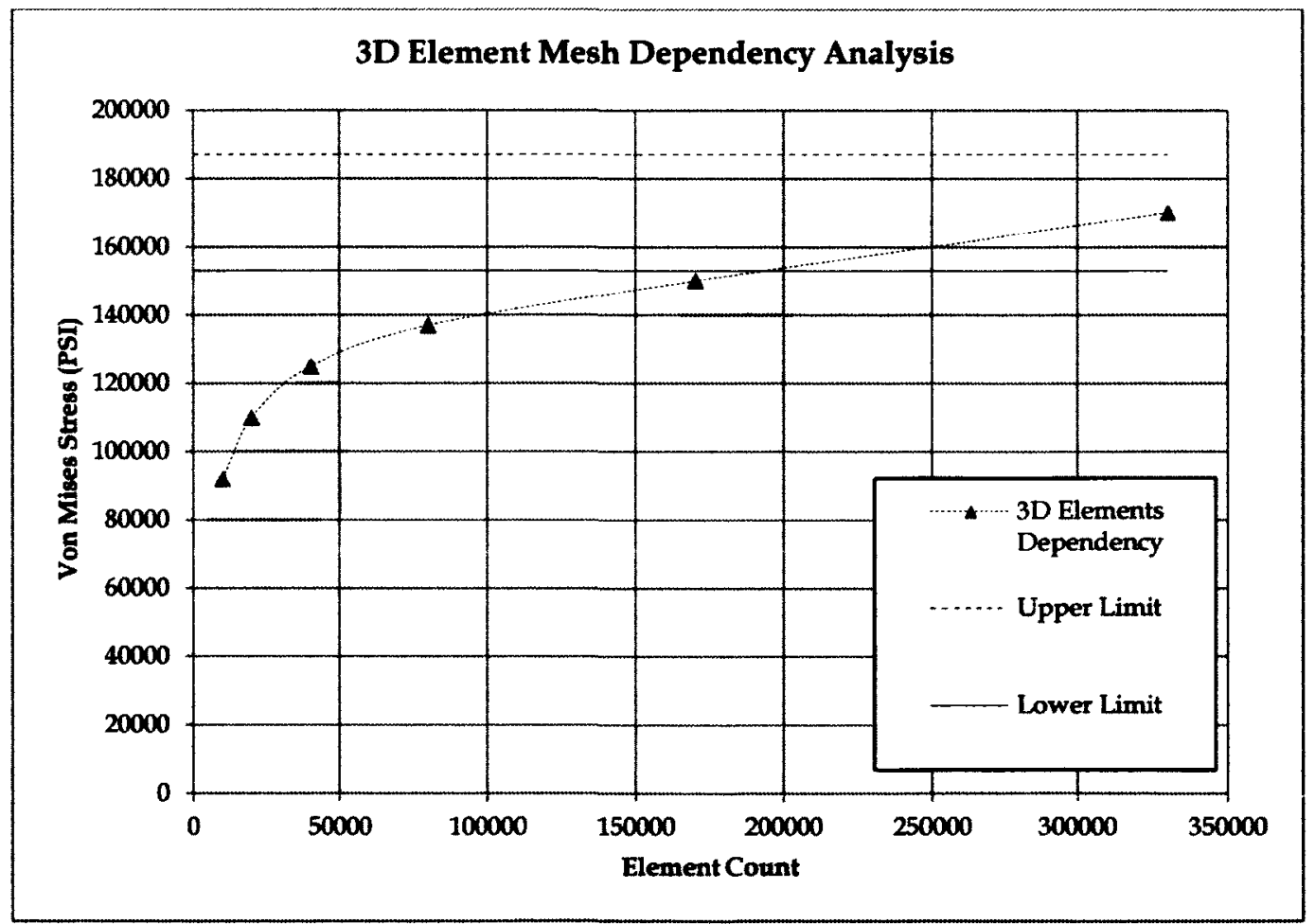

Figure 67: 3D Stress Element Mesh Dependence Analysis 


\subsection{Conventional shell model (Model 1)}

While designing composite components, there are a few parameters that may be changed in order to affect performance characteristics of the components, these include: fibre material, resin material, ply count, ply orientation and geometry. For this model in particular, three parameters where varied for coupon design optimization: patch shape, patch geometry, and ply orientation. While testing the effect of each parameter, all other parameters were held constant. Of all tested parameters, those yielding the highest Von Mises stresses on the adhesive layer where chosen and then carried through to the next model.

There are several different coupon designs that would have induced disbond within the adhesive layer under static load; all of which would have been adequate for the primary objective of this study, but only one was utilized. A starting point for the design of the composite-to-composite coupons was based on the composite-to-metallic design described in section 3.1.2. The exact dimensions and geometry of coupons described in section 3.1 .2 were duplicated for an initial design within ABAQUS and is shown in Figure 68.

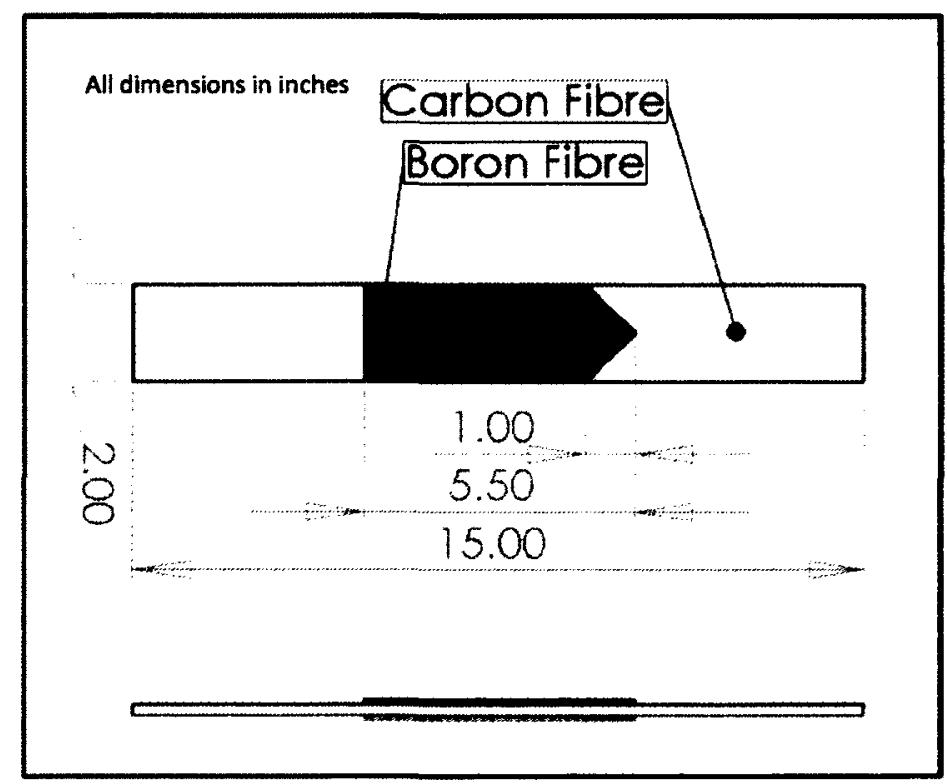

Figure 68: Smart Patch Phase 1 \& 2 Coupon Design (measurements in inches) 
Three different patches were constructed within $A B A Q U S^{\bullet}$; all were created as 3D deformable shell parts, as this was the part that was mapped with shell elements. Patch 1 (Figure 69(a)) is similar to that of coupon set 2 . This patch has a symmetric center spike that is designed to create a stress concentration zone at the tip of the patch. Patch 2 (Figure 69(b)) was chamfered to one side. Patch 3 (Figure 69(c)) was completely an unsymmetrical patch that was used to evaluate the effects of unsymmetrical bending on the adhesive layer. All three designs attempted to create a rapid change in geometry through which transfer of load was not done efficiently and local stresses were allowed to rise significantly. All three geometrically different patches have undergone some level of facial partitioning as shown in Figure 69 . These partitions were created so that meshing using shell elements later within the assembly may be done as a structured mesh.

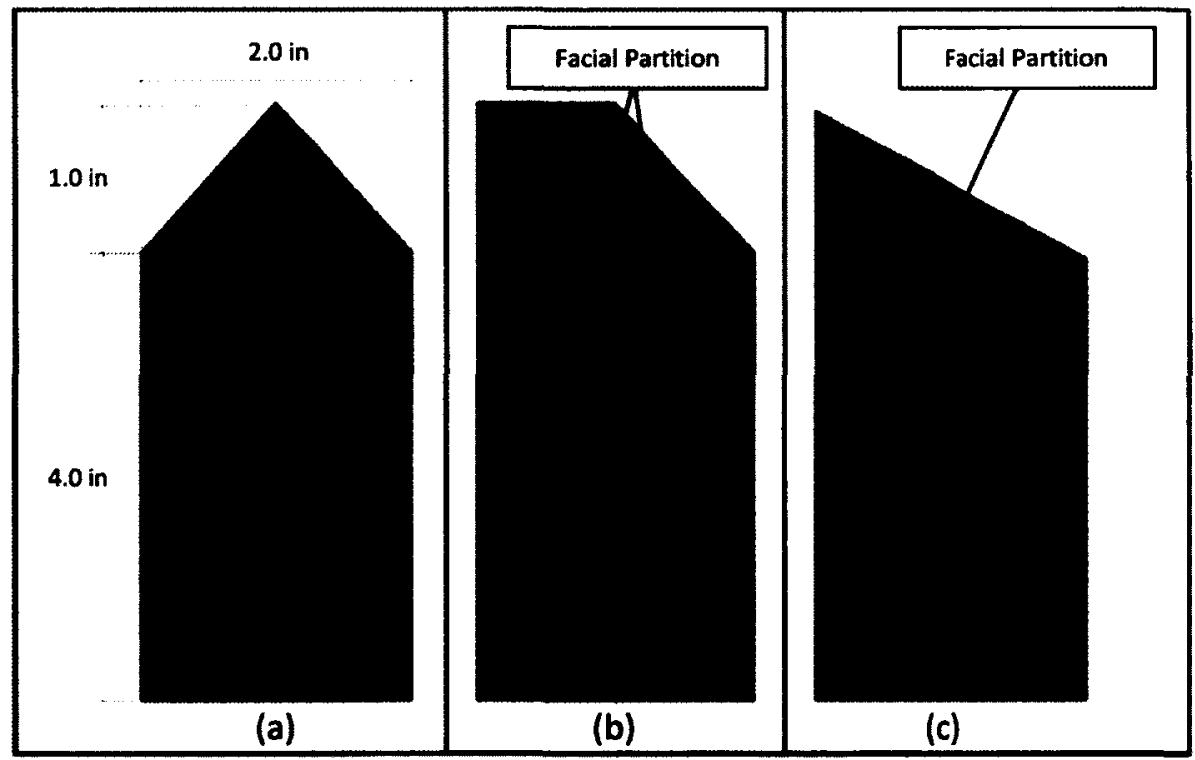

Figure 69: Patch Part: (a) Spike Patch, (b) Chamfer Patch and (c) Unsymmetrical Spike Patch

Three different adhesives were also constructed within ABAQUS*; all were created as 3D deformable solid parts with a thickness of 0.007 inches and similar dimensions as those used for the patches. The adhesives were not partitioned in the same manner as the patches, as the 3D 
stress elements used for these parts maintained better structure as compared to shell during geometric changes. Figure 70 shows all three adhesive designs.

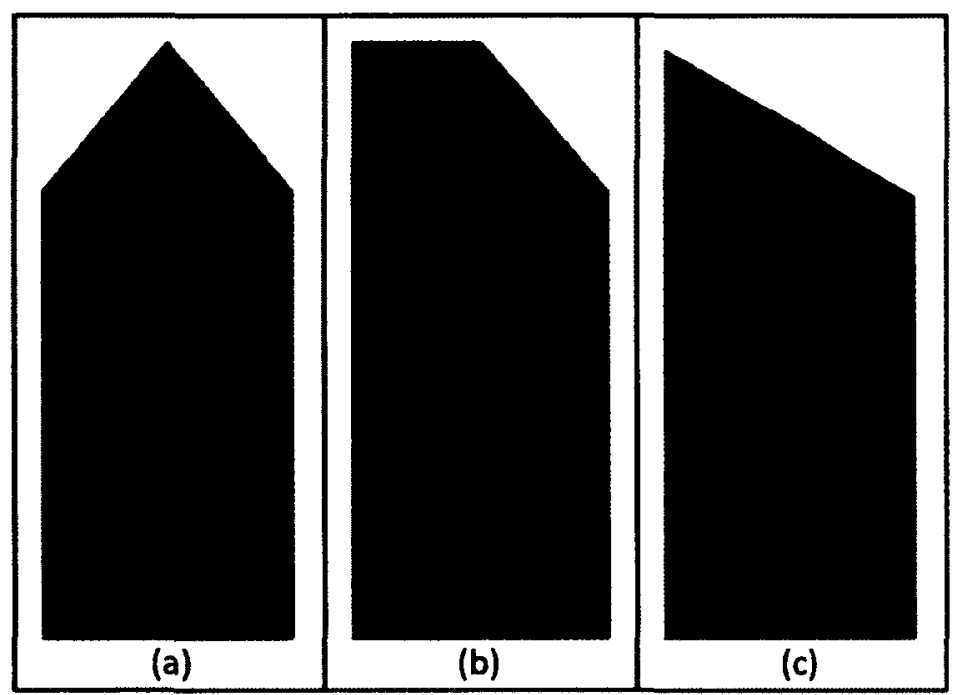

Figure 70: Adhesive Part: (a) Adhesive for Patch 1, (b) Adhesive for Patch 2 and (c) Adhesive for Patch 3

Three substrates were constructed; they were created as 3D deformable shell parts. Similar partitions were made to the substrate as those made to the patches, as they use the same element type. In addition to the partitions made for the patch, two additional partitions were made on both the top and the bottom of the substrate. These partitions were done to act as the area that would be subject to the gripping force of the Material Test System (MTS) test frame. These portions were made as the full width of the substrate and measure 3 inches in length. Figure 71 shows the substrates along with the facial partitions. 


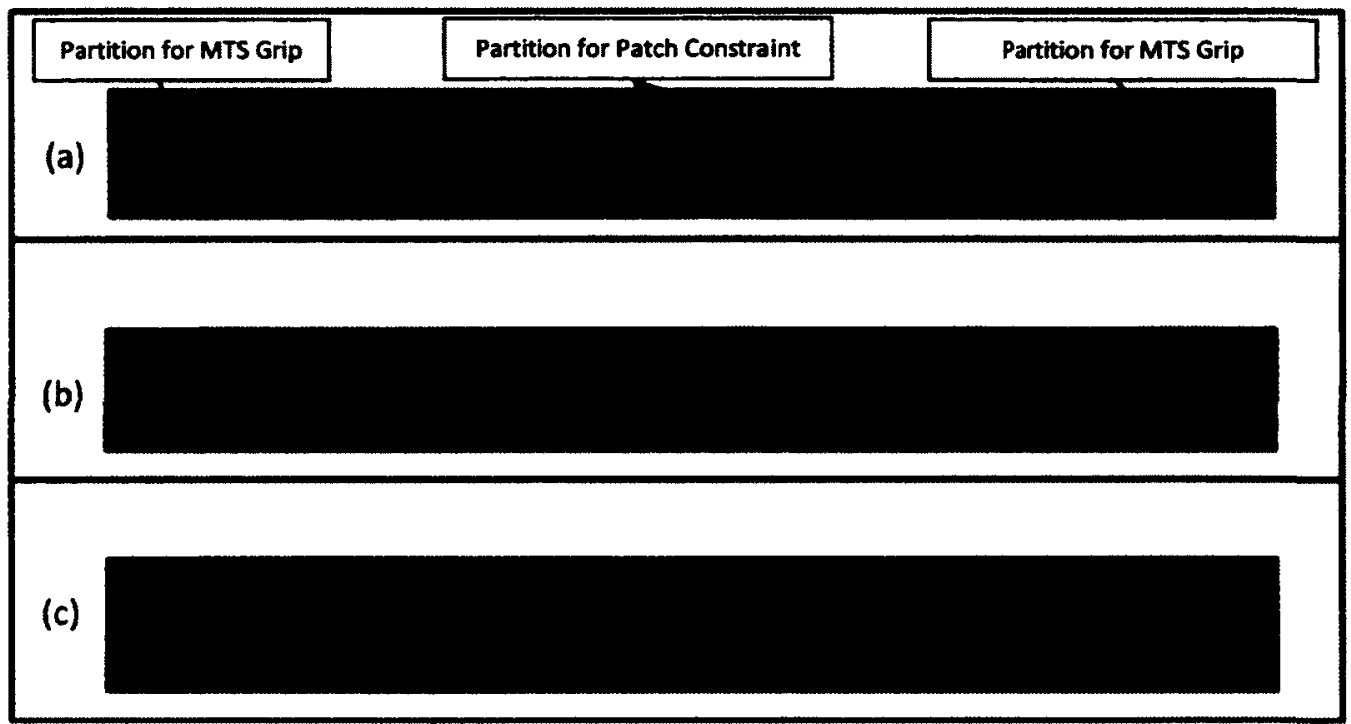

Figure 71: Substrate Part: (a) Dissections to Accommodate Patch 1, (b) Dissections to Accommodate Patch 2 and (c) Dissections to Accommodate Patch 3

Three assemblies were constructed, one for each of the different variations of model 1 (i.e. different patch designs). Assemblies were produced by importing each component into the assembly window and creating positional constraints. Figure 72 shows mating surfaces and edges utilized during the assembly process. Surfaces A \& B and G \& $H$ were made to coincide as shown in Figure $72(b)$, both surface constrains ensured that the adhesive film is in direct contact with both the substrate and the patch. Edges $C \& D$, and E \& F were made to coincide and have a separation distance of 5.0 inches, respectively, as shown in Figure 72(a). Edge constrains insured proper location of the patch along the substrate. 


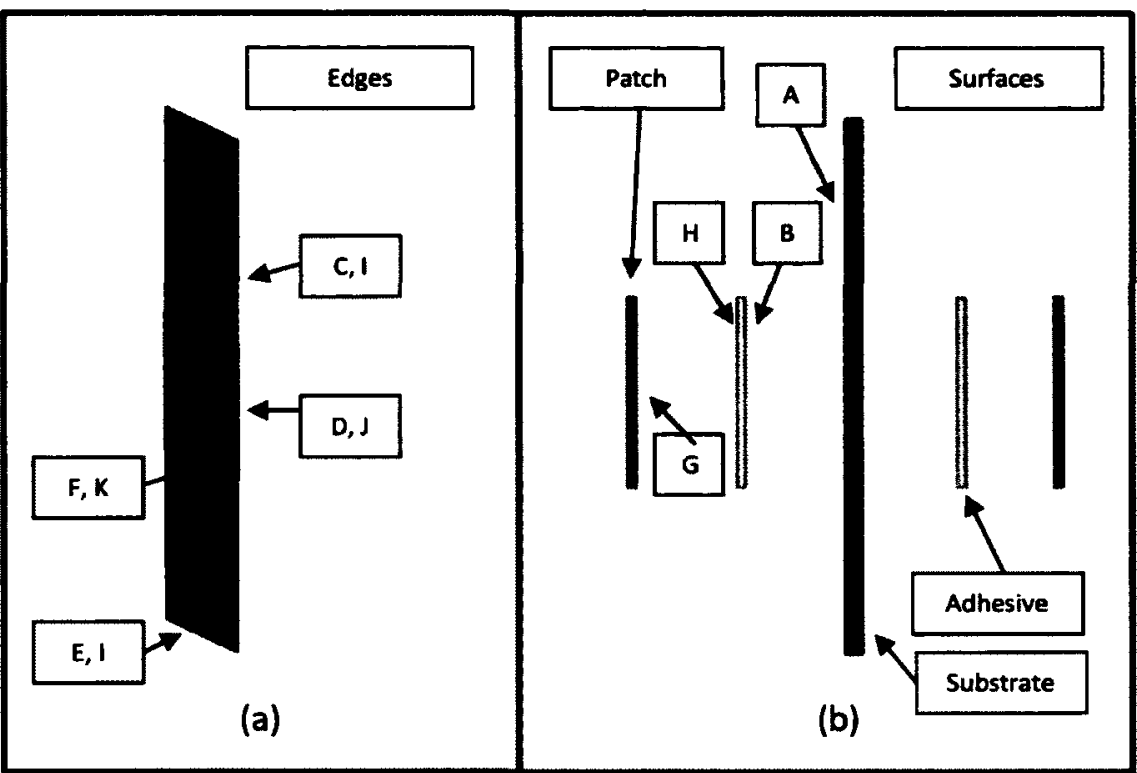

Figure 72: Assembly Position Constraints: (a) Isotropic View and (b) Side, Exploded View

In addition to position constraints, tie constraints were introduced into the model. Tie constraints were utilized to join nodes found on surface $A$ with nodes on $B$ and nodes on $H$ with nodes on G. Through this tie constraint, boundary conditions were shared between two adjacent surfaces and ultimately identical displacements.

Partitions shown in Figure 71 measuring $2.0 \times 3.0$ inches were subjected to both the load and boundary condition as shown in Figure 73 . These sections were created to simulate the grips which were used for load application. The applied load was created as a traction load and was applied to both sides of the substrate (front and back). The traction load was created with a uniform distribution. The boundary condition was set on the bottom section of the coupon (front and back); this boundary condition was created such that it did not allow motion along any of the three axes. 


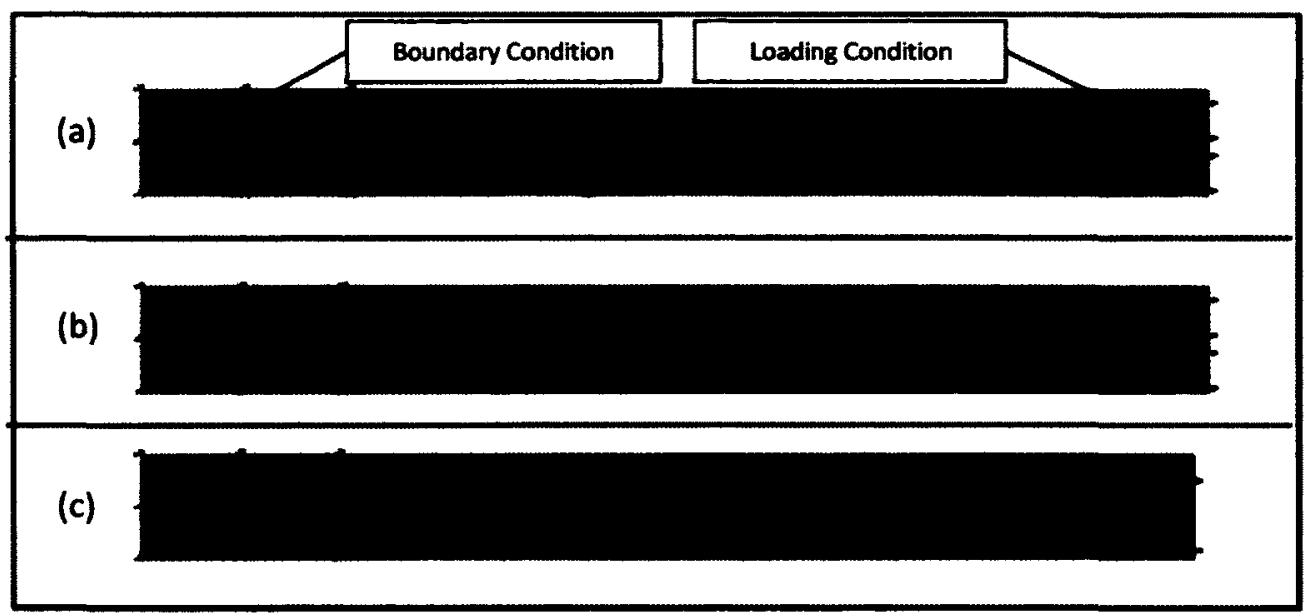

Figure 73: Loads and Boundary Conditions: (a) Model 1, (b) Model 2 and (c) Model 3

\subsection{Variant patch designs and analysis}

All three patches revealed that the introduction of a pointed patch tip results in increased local stress within the adhesive. All three patches were evaluated under the same load and same layup for both the substrate and the patch. Figure 74 show the results of the Von Mises stresses as obtained from FEA. Figure $74(a)$ and Figure $74(b)$ both show that introduction of a rapid change in geometry within the patch results in an increased Von Mises stress within the adhesive. The increase in stress occurs at the same location as that of the patch tip. However, for the third patch design (Figure $74(\mathrm{c})$ ), the highest stress zone does not occur at the tip of the patch, rather it occurs in the corners. Each value of stress was compared to patch 1 and it was found that patch 2 yielded stresses which were $1.8 \%$ lower than that of patch 1 while patch 3 was found to be $24.1 \%$ lower than that of patch 1; values are listed in Table 3. Patch 1 was chosen as the optimal design and carried forward through the next stages of the analysis. 


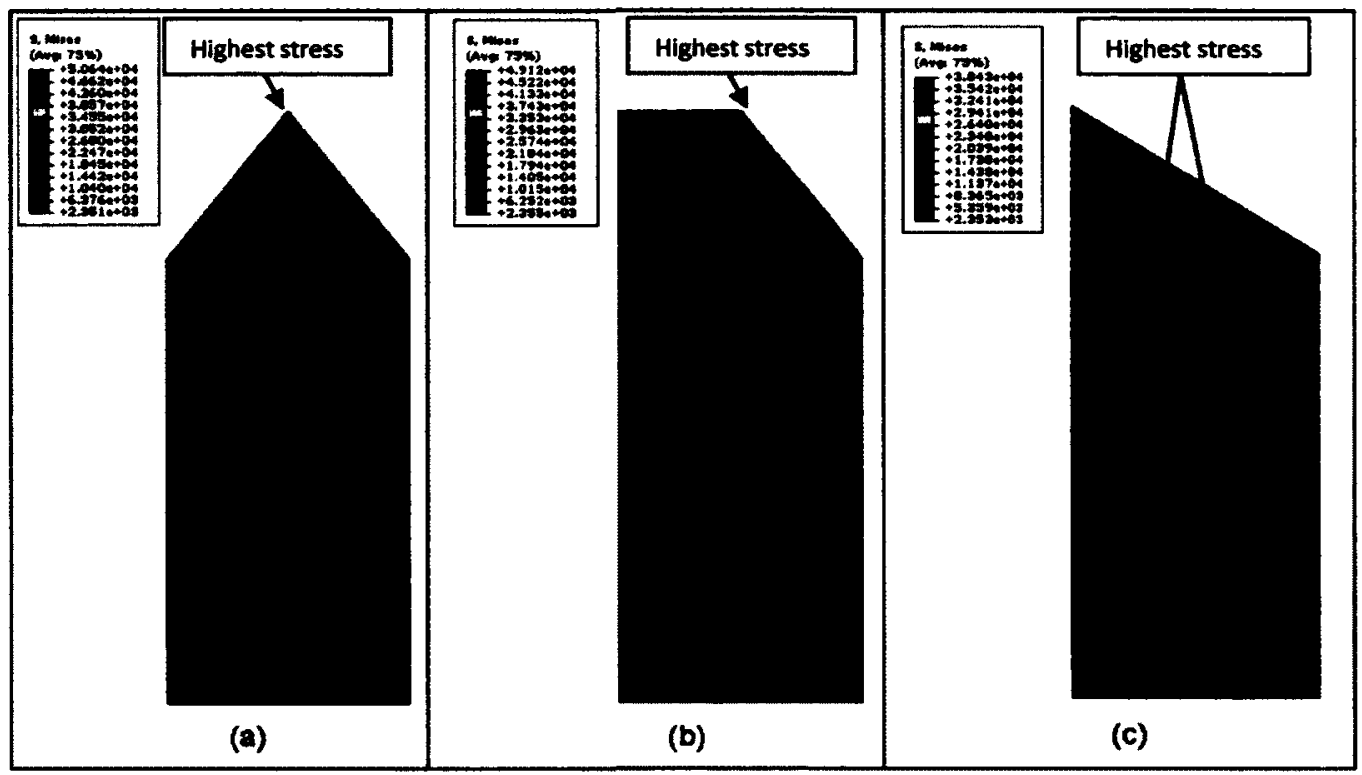

Figure 74: Von Mises Adhesive Results: (a) Spike Patch, (b) Chamfer Patch and (c) Unsymmetrical Spike Patch Table 3: Von Mises Stress Comparison

\begin{tabular}{|c|c|c|}
\hline Patch & Von Mises (KSI) & (Comparison to patch 1) \\
\hline 1 & 50.6 & N/A \\
\hline 2 & 49.1 & $-1.8 \%$ \\
\hline 3 & 38.4 & $-24.1 \%$ \\
\hline
\end{tabular}

\subsection{Variant patch geometry and analysis}

Based on results from section 3.1.3.3.1.1, patch 1 was selected and geometry was varied to determine the effects of the patch vertex angle. To analyze the effects of the patch vertex, the patch geometry was changed for multiple analyses. The analysis was conducted for different vertex angles varying from $45^{\circ}$ to $90^{\circ}$. It was found that as the angle increased, the $S_{12}$ (directions noted within Figure 75) on the adhesive layer decreased as shown in Figure 75. Based on this analysis, the vertex angle was chosen to be $45^{\circ}$. 


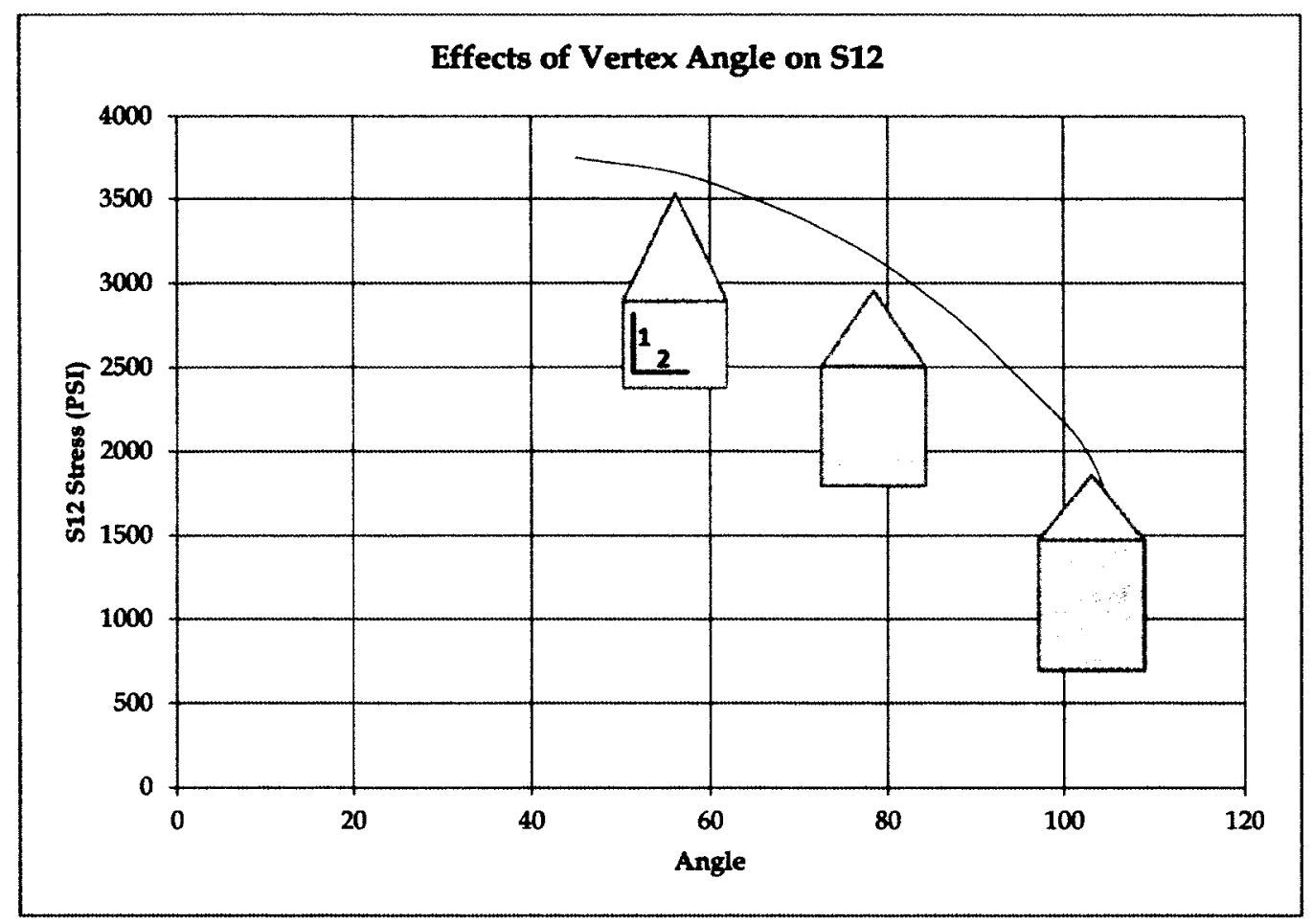

Figure 75: Effects of Spike Angle on the S12 Adhesive Stresses

\subsection{Variant ply orientation analysis}

The final parameter that was examined was the ply orientation within the substrate. Change in ply orientation changes the stiffness and creates a larger difference between the stiffness of the patch and the stiffness of the substrate. The study was done by taking a single ply and rotating it from $0^{\circ}$ through $90^{\circ}$ while maintaining the same load magnitude and direction. Results showed that as the stiffness of the substrate decreased with respect to the patch, the $S_{12}$ stresses within the adhesive increased. These results were found by examining Figure 76. 


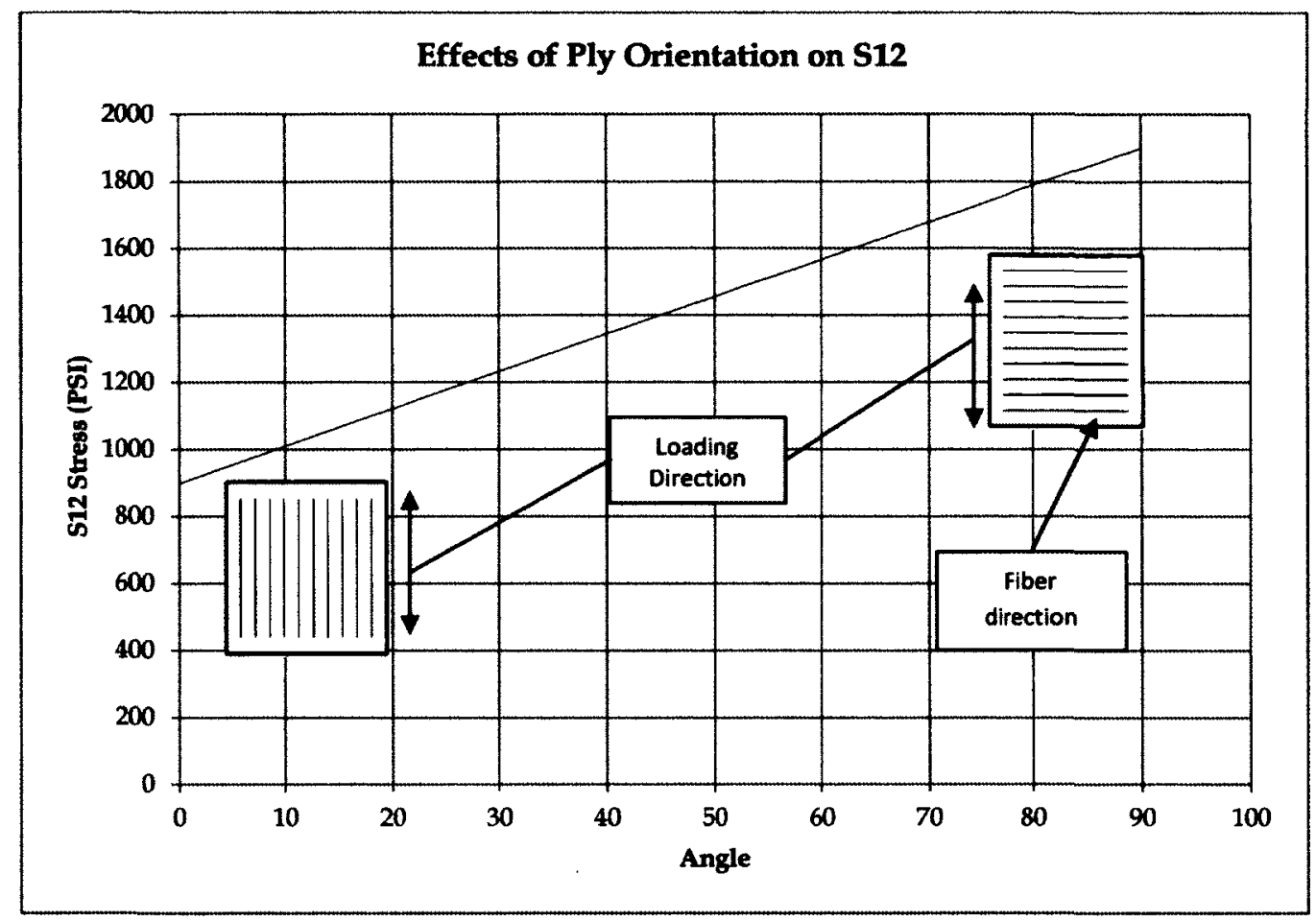

Figure 76: Effects of Ply Angle on S12

\subsection{Summary of results}

FEA results using the simplified conventional shell elements revealed that a symmetric patch with a vertex forming $45^{\circ}$ yields the highest $S_{12}$ stress within the adhesive layer. Also, the greater the difference of stiffness between the patch and the substrate the greater stress build up within the adhesive layer.

Using these results, the substrate layup was chosen to be of only $\pm 45^{\circ}$ and was set to $[45,-45$, $45,-45]_{s ;}$ this was chosen because it yields the lowest stiffness along the loading direction as well as ensuring that the substrate can support the applied load without failure by matrix shearing. The patch layup was chosen to be made of primarily $0^{\circ}$ and was set to $[0,0,45,-45]_{s}$. The $0^{\circ}$ yields greatest stiffness along the loading direction. The introduction of $\pm 45^{\circ}$ was done so that the patch has some strength perpendicular to the applied load. 


\subsection{Continuum shell model (Model 2)}

The continuum shell model was the second model that was developed for analysis of the coupon design. Due to the continuum shell element properties, this model was only used to determine individual ply stresses. These stresses were then compared to the results of the conventional model to determine the model's efficiency and accuracy.

The continuum model is very similar to the conventional model in its setup; however, there are small differences that have been carried out in order to accommodate the continuum shell element meshing. This section only discusses differences between both models and compares their results.

Both the patch and substrate were constructed within ABAQUS ${ }^{\star}$ as $3 D$ deformable solid parts within the continuum shell model. 3D deformable solids allow for through thickness assignment of elements as opposed to shell parts which were used in conjunction with the conventional shell elements. The constructed patch was that of Type 1. Remainder patch designs were discarded as they resulted in lower shear stresses in the presence of the same applied load.

Implementation of the continuum elements (SC8R) was done by defining a sweep mesh. A sweeping mesh is the application of elements in layers; this was done to layer multiple planar element sheets through the thickness of the component. The layering of planar element sheets was done through the thickness of the component as shown in Figure 77 , which had a predefined 8 nodal count along the edges ( 1 node for each ply). This sweep was done for both the substrate and the patch. By stacking 8 layers of elements, stresses along the $x-y$ coordinates were evaluated for each individual ply. 


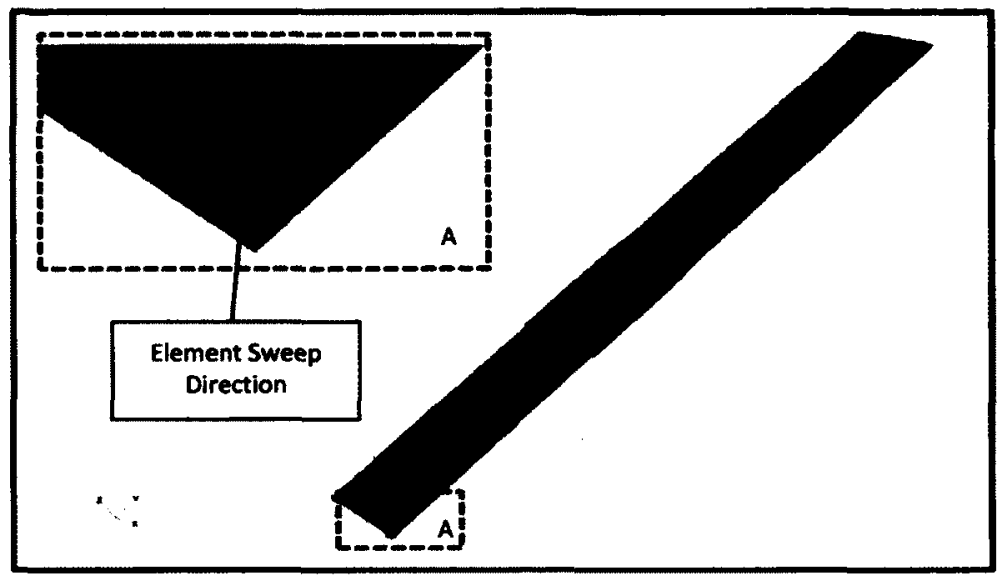

Figure 77: Mesh Sweep Direction

\subsection{Results}

After evaluating both the conventional and continuum shell models, it was found that both models yielded similar results when evaluated under the same condition as shown in Figure 78. Both models were found to converge to two different Von Mises stresses while having $10 \%$ convergence criteria. However, the difference between the two converged stresses was approximately $2 \%$. The $2 \%$ difference is small and may be neglected. Both models employed the same coupon design and geometry, the only difference between the two models was the element type and therefore computational resources required for the analysis was different. Continuum elements (SC8R) have twice the amount of nodes within each element as compared to that of the conventional elements (S4R). For that reason, the continuum model requires additional resources to compute the same job. In addition to the number of nodes, S4R are designed specifically for use with composite lamina and therefore utilizes assumptions that make computation very efficient. 


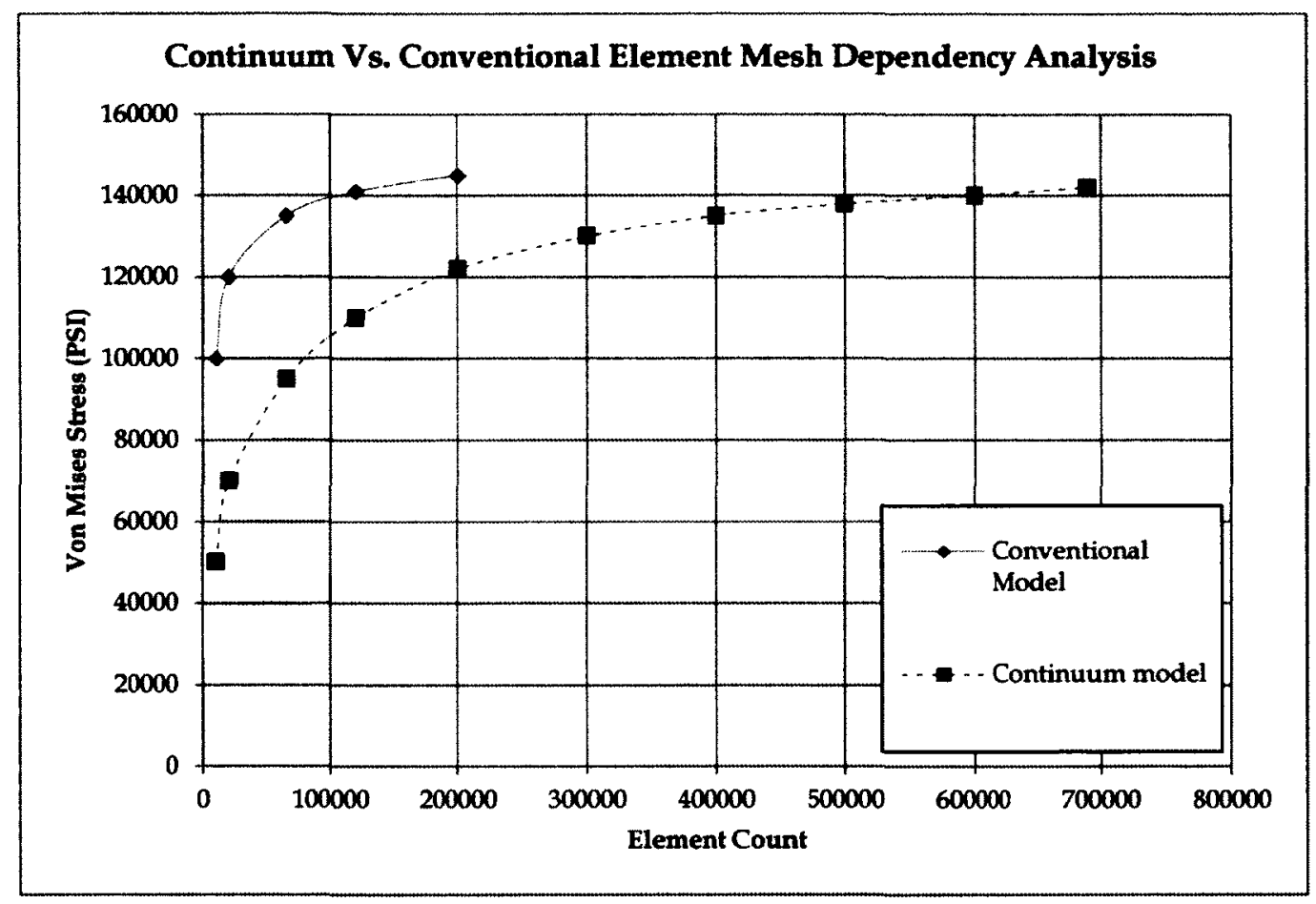

Figure 78: Comparison of both Continuum and Conventional Shell Models

\subsection{Solid model (Model 3)}

Unlike the previous two models, the solid model utilized 3D stress elements which allowed for the computation of stresses in all directions. For this reason, thermal residual stresses that were introduced during the bonding process were computed using this model. This model was setup to have 3D deformable solid parts similar to those used within the continuum model. However, unlike the continuum model, this model utilized different elements and was subjected to two load applications: a static load and a thermal load. To control which load is applied to the system first, an additional step was added. The purpose of the first step was to apply the thermal load to the system, after which the second step was to apply the static load.

During bonding, heat is applied to the system causing the temperature to increase to $250^{\circ} \mathrm{F}$, causing carbon fibres to shrink and the boron fibres to expand; this is a result of a negative thermal expansion coefficient and a positive thermal expansion coefficient, respectively. The 
FM73 adhesive cures at $250^{\circ} \mathrm{F}$ and once the system is allowed to cool, the coupon will not return to its original state and therefore thermal residual stresses are developed within the system. Simulation of this within FEA was done through the creation of a boundary condition which set the entire model at an equilibrium temperature of $250^{\circ} \mathrm{F}$; in addition to this boundary condition, a second boundary condition was created within a second step. This second boundary condition imposes an equilibrium temperature of $74^{\circ} \mathrm{F}$. By creating these two boundary conditions, ABAQUS ${ }^{\bullet}$ is able to calculate the displacements of each node due to temperature change. For this problem, only the steady state result was of interest; therefore imposing an initial and final temperature is adequate for this problem as calculated displacements due to expansion and contraction were used to calculate stresses.

\subsection{Results}

Figure 79 shows stress distribution in the $x-y$ plane for all components after application of both thermal and static loads. Stress distribution shows that the rapid change in geometry (i.e. patch vertex) affects the stresses locally as expected. The applied force region in Figure 79 shows a gradient of stress; in real life this would not occur as the area in the grip would not be permitted to deform. A similar stress distribution does not occur in the applied boundary condition region because nodes are not permitted to strain within all directions. A boundary condition imposing relative location of nodes to neighbouring nodes in the applied load region was not imposed as stress and strain distribution at the loading area had no impact on the area of interest. Figure 80 shows stress distribution within the $x-y$ plane for both the patch and the adhesive. Both the patch and adhesive stress distributions appear to be mirrored along the center line of the coupon, with a positive stress side and a negative stress side; both equal in magnitude. This symmetry in distribution is due to both the layup of the substrate and the patch. Through the 
use of a substrate with a $[45,-45,45,-45]_{s}$ and a patch with a $[0,0,45,-45]_{s}$ layup stresses are not expected to be highest at the vertex, rather at the area of the highest stiffness. The area of highest stiffness occurs at the $45^{\circ}$ angle and therefore the stresses at $90^{\circ}$ separation occur in the opposite magnitude as the ply is weakest in that direction. 


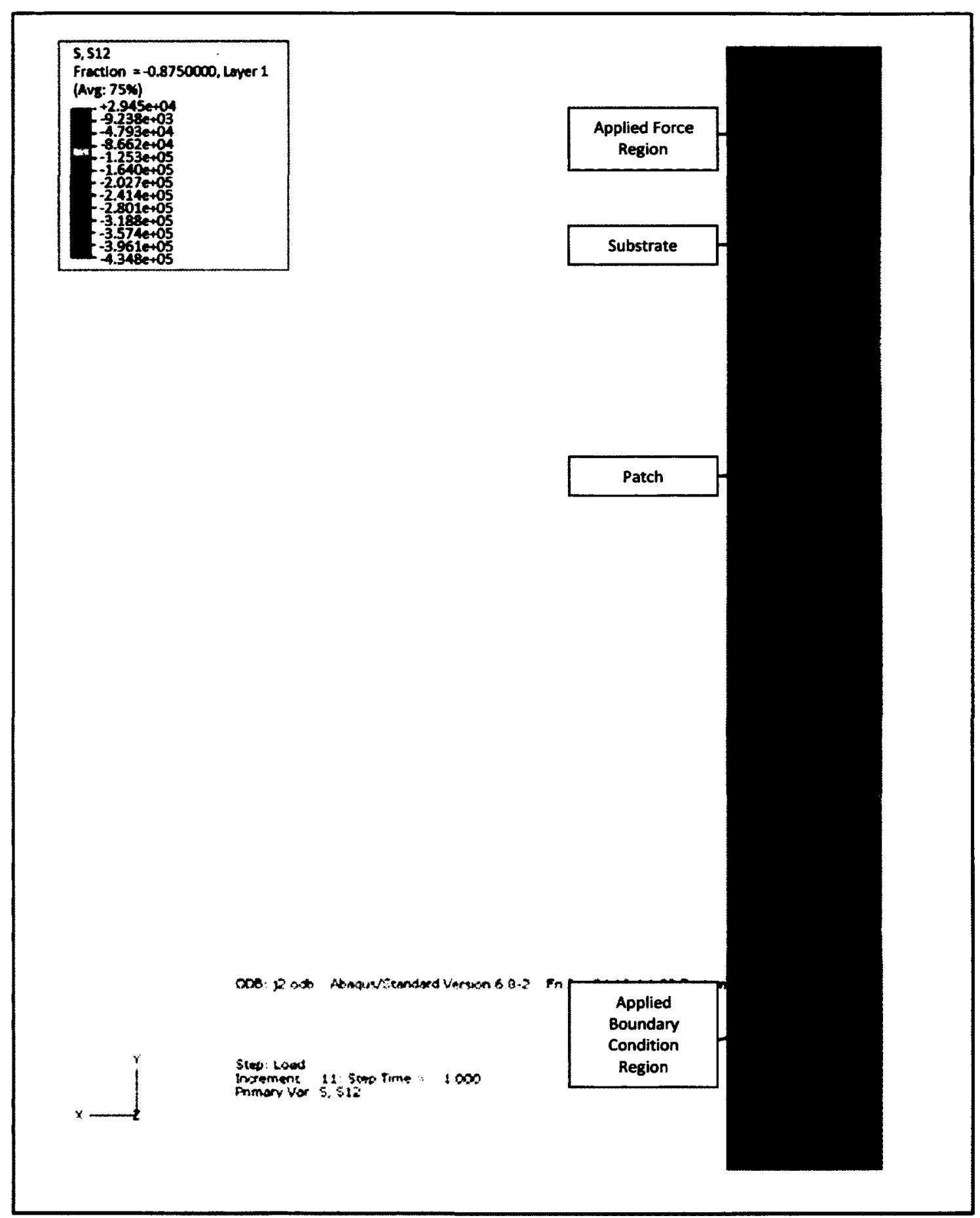

Figure 79: Full Model Stresses 


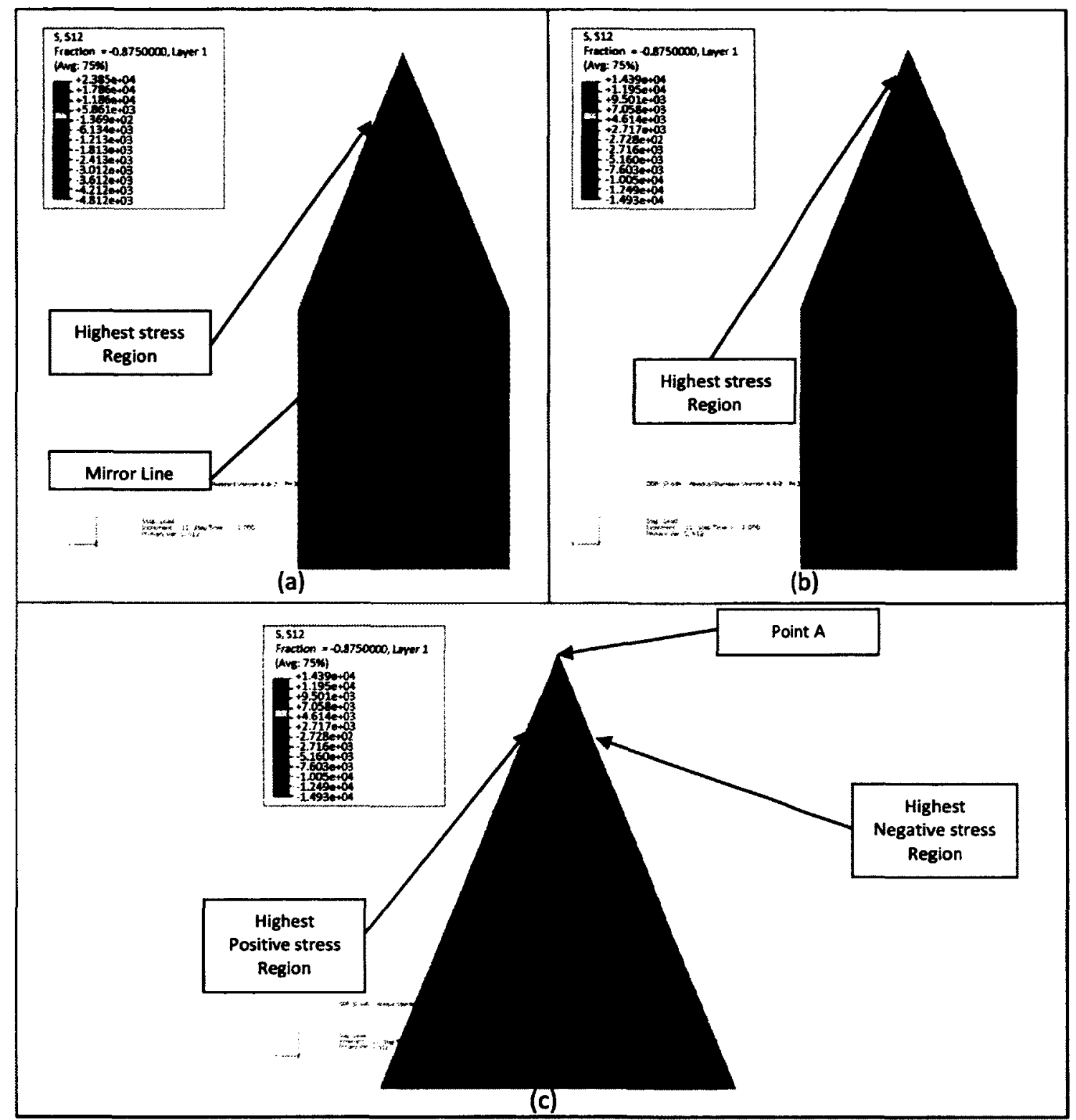

Figure 80: Stresses Distribution: (a) Patch, (b) Adhesive and (c) Magnified Adhesive

To determine the effects of thermal residual stresses, a single node was examined for stress after the application of the heat load and after the application of the mechanical load. The element in question is located at the tip of the adhesive and is located in an area of high stresses as shown in Figure 80 (c) Point $A$. Figure 81 shows the change of stress in the $x-y$ plane at point $A$ against the two step intervals. Step intervals range from 0 to 2 ; this represents the two sequences of events allocated to each computational event within ABAQUS ${ }^{\bullet}$. Between 0 and 1 
(step 1), the coupon is subject to a change of temperature while from 1 to 2 (step 2) represents the application of mechanical load. During step 1 , the coupon is subject to shrinkage which simulates the cooling down of the coupon after adhesive solidification. At the end of step 1, shrinkage of the coupon yields a negative stress as expected and is equal to -100 PSI at point $A$. During the application of load, the thermal residual stresses are overcome and local stress values climb to 7,118 PSI. These results indicate that the applied load will overcome the thermal residual stresses and results in a bond failure.

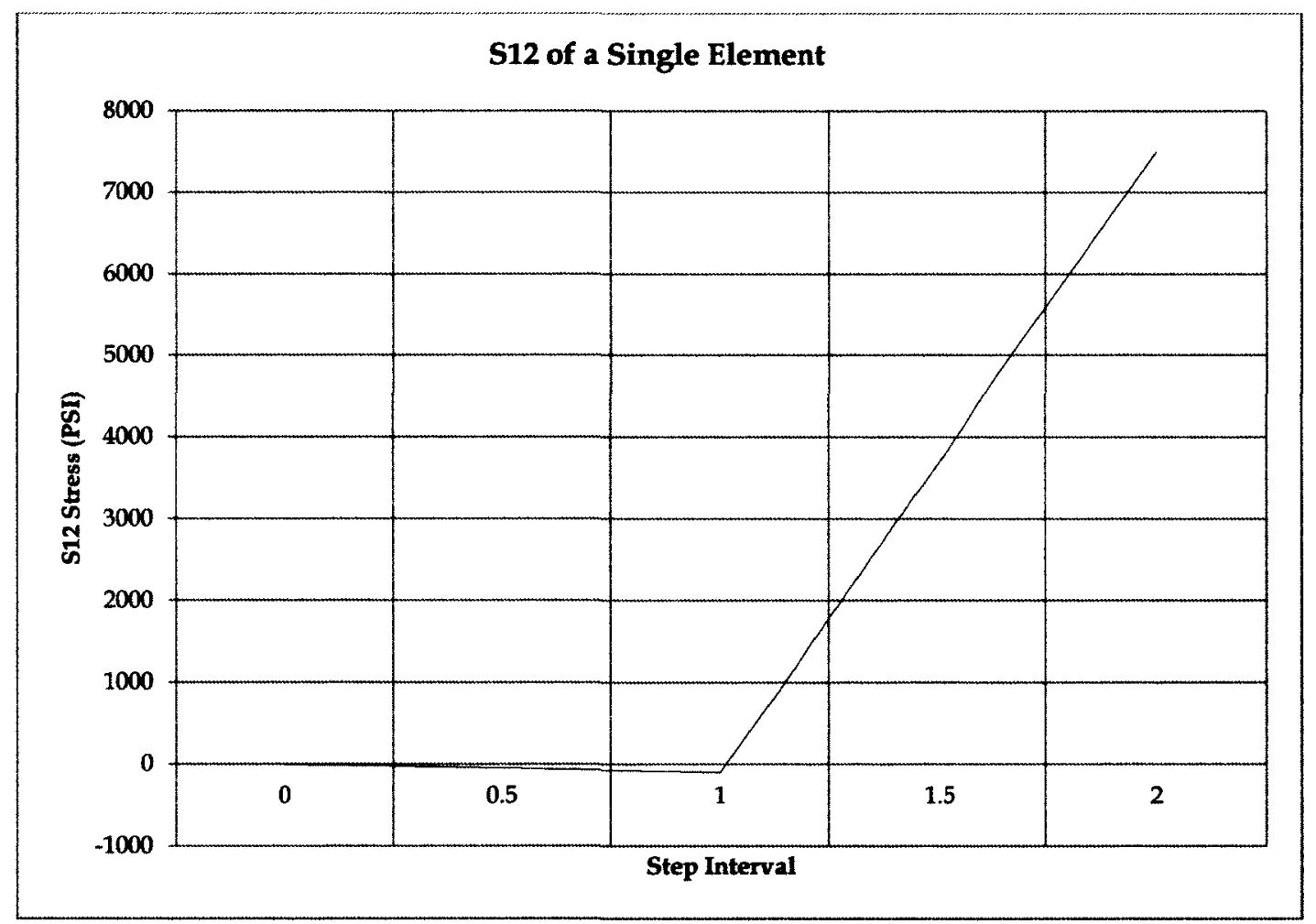

Figure 81: Change in Stress over Application of Heat and Load

A failure criterion for this study is defined as a failure within the adhesive layer, which shears at 6,680 PSI. Examination of both Figure 80 and Figure 81 shows that there will be enough shear stresses that will cause failure. These results are based on a mechanical load of $15,000 \mathrm{lb}$ which overcomes the thermal residual stresses. However, there are many factors to be considered when loading composites, especially those not having $0^{\circ}$ fibres along the loading direction. 
Composites having non-zero degree fibres experience failure modes other than fibre breakage and therefore the strength of the component is not as high as expected. For this reason, the coupon was later loaded incrementally to determine its ultimate strength. In addition to the strength of the coupon, SHM instrumentation played an important role for this study as sensors must withstand the strains for data acquisition. Sample coupons were tested to determine the coupon ultimate strength as well as sensor adhesion strength. These loads determined the exact test matrix for all coupons that were manufactured for this project which is described later in the testing chapter, Chapter 4.

\subsubsection{Carbon fibre substrate manufacturing}

The manufacturing procedure used for the substrates involved accurate ply cutting of CYCOM 5276-1 G40-800 at appropriate angles, stacking plies at the correct orientation, placing the layup under vacuum and finally curing the layup. All of these steps within the procedure are described in greater detail within the following subsections.

\subsection{CYCOM 5276-1 G40-800 prepreg tape cutting}

FEA computational analysis revealed that disbond between adhesively bonded components will occur as a result of sufficient stress build-up within the adhesive layer. Substrate strength for this study was achieved by two methods: the material property and the component layup. For this substrate, the optimum layup was found through FEA analysis to be $[45,-45,45,-45]_{s}$. Deviations from those angles will result in either a higher or lower stiffness; this will affect the material strain in the presence of an applied load. For that reason, it was very important to maintain ply orientation. Cutting plies and stacking them within $\pm 2^{\circ}$ was difficult for small coupons as fibres are subject to skewing during handling. To minimize fibre skewing, handling of 
prepreg plies was minimized; this is done by creating oversized laminates from which the final substrates were machined after laminate curing. The manufactured oversized laminate measured $16.0 \times 15.0$ inches as shown in Figure 82. Each large laminate produced 6 substrates measuring $15.0 \times 2.0$ inches as shown in Figure 82 .

It is known that edges of any laminate will have many inconsistencies during manufacturing. Inconsistencies within the edges are a result of resin build-up at the edges caused by resin bleed. All edges consequently have uneven thickness and therefore inconsistent fibre volume fraction ratios. Elimination of this edge effect was done by ensuring the laminates were made oversized by 0.5 inches all around the perimeter. The additional 0.5 inch perimeter was removed during machining; all material found inside the 0.5 inch perimeter was consistent in both volume fraction and thickness.

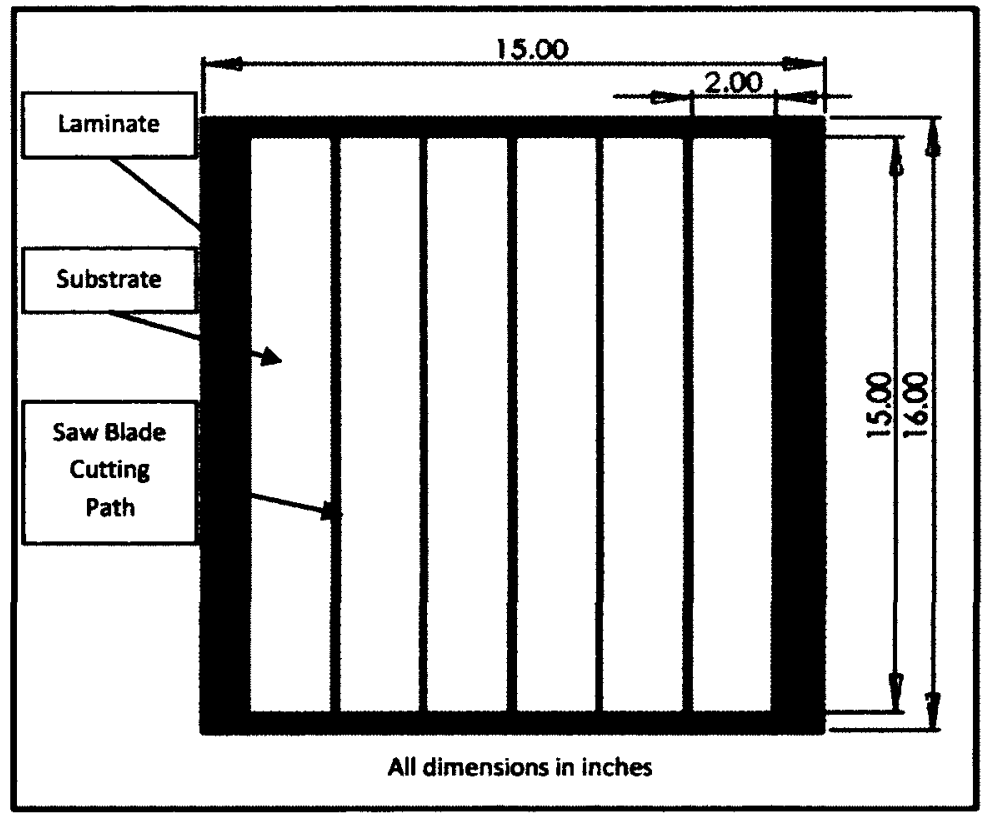

Figure 82: Laminate-Substrate Schematics

To manufacture the $16.0 \times 15.0$ inch laminates, 8 plies were cut. For each laminate 4 plies were cut at $+45^{\circ}$ and the other 4 at $-45^{\circ}$. Correct ply angles were ensured through the use of cutting 
templates as shown in Figure 83. Initially, a roll of CYCOM 5276-1 G40-800 prepreg tape is rolled out with fibre orientation as shown in Figure $83(a)$. Cutting of plies at $45^{\circ}$ was done by placing a $45^{\circ}$ triangle made of aluminum along the edge of the roll which is assumed to be straight as shown in Figure $83(\mathrm{~b})$. Through the use of this triangle, a $45^{\circ}$ edge was created. A perpendicular edge was then created through the use of a straight edge as shown Figure $83(c)$. Both the triangle and the straight edge formed a border for the $45^{\circ}$ plies. The plies were then cut from the roll with the correct angle as shown in Figure 83(d).

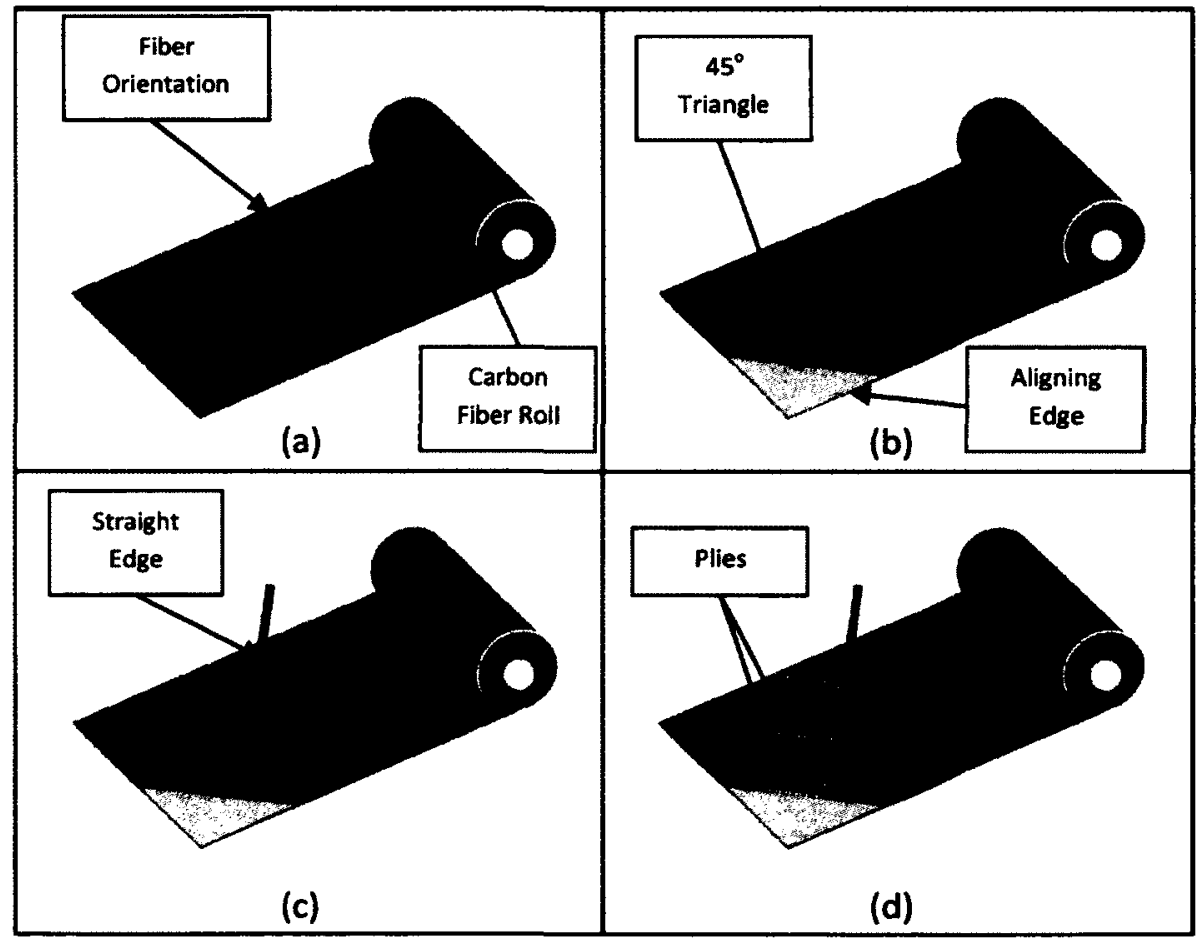

Figure 83: Ply Cutting Procedure: (a) Carbon Fibre Roll, (b) $45^{\circ}$ Alignment, (c) Straight Edge, and (d) Ply Cuts

Ply stacking operations were carried out to create the laminate. Ensuring ply orientation was done by using a right angle ruler, while fixing it to the working area and placing each ply corner within the corner of the ruler as shown in Figure 84(a). Through the use of this procedure, edges which were defined through the cutting procedures will coincide with edges of the ruler and consequently the edges of adjacent plies. Due to the sticky surface of the prepreg, air becomes 
entrapped as each ply is placed on top of the previous; this entrapped air must be removed. Entrapped air creates excess voids which affect both the strength and the stiffness of the laminate. Removal of the air was done through two operations: rolling and debulking. Rolling is a process in which a standard roller (similar to a dough roller) is rolled over the surface of each ply after its placement on the previous ply as shown in Figure $84(b)$. By attempting to flatten the surface of each ply, entrapped air is squeezed out towards the edges. It was very important that the roller starts each operation at the center of the layup and moves towards the edge of the layup as shown in Figure 84(b), otherwise the operator moves air from the edge to the center of the laminate. The roller was used in between the placement of each ply. The second process for the removal of entrapped air was debulking; this is a process in which a specialized pressure distribution table is used to squeeze the laminate between large flat surfaces. The debulking process was used for every 4 plies and not after the addition of each ply. Through the application of pressure on the two surfaces of the laminate, air is forced out of the laminate. However, the application of pressure was done incrementally as it provided adequate time for air to flow out of the layup. The gradual pressure application procedure is given in Table 4.

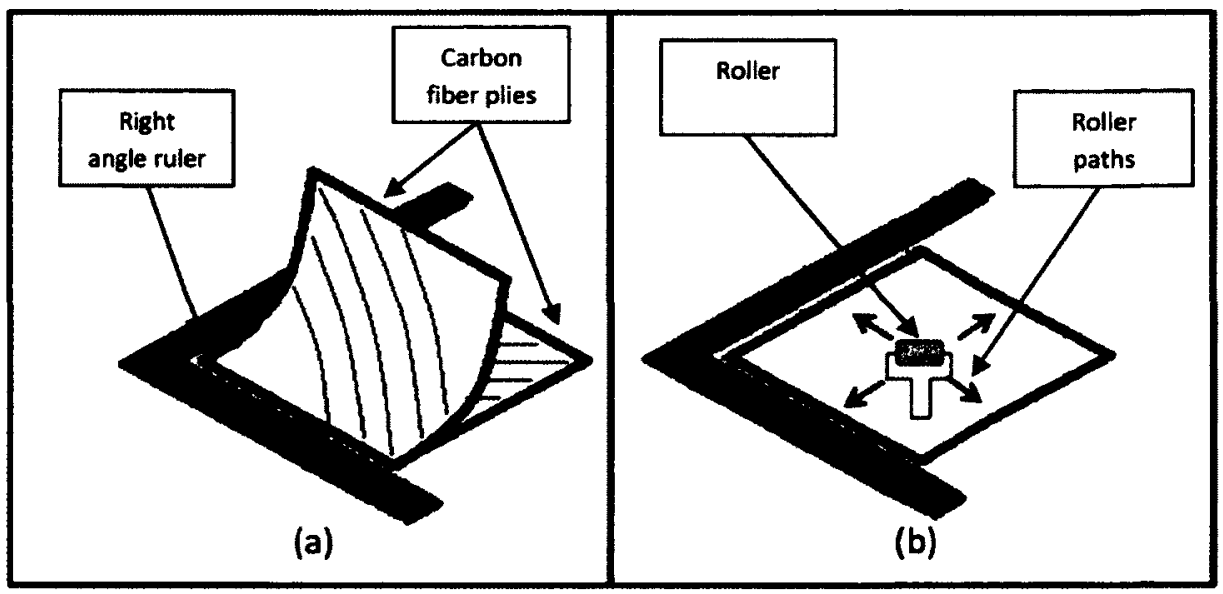

Figure 84: Layup Procedure: (a) Layup, and (b) Entrapped Air Removal 
Table 4: Debulking Procedure

\begin{tabular}{|l|l|}
\hline \multicolumn{1}{|c|}{ Vacuum Pressure } & \multicolumn{1}{|c|}{$\pi \mathrm{mo}$} \\
\hline 5 PSI & 5 minutes \\
\hline 10 PSI & 5 minutes \\
\hline 15 PSI & 5 minutes \\
\hline 20 PSI & 5 minutes \\
\hline 25 PSI & 5 minutes or more \\
\hline
\end{tabular}

\subsection{Laminate bagging}

Laminate bagging is the step in the manufacturing process in which the uncured laminate is sandwiched between two large aluminum plates (a tool plate and a caul plate) and placed within a vacuum bag. This was done to prepare the laminate for the autoclave oven; which applied both heat and pressure for laminate curing. Proper bagging was crucial as it defined the laminate surface quality, mechanical properties and volume fraction.

The laminate was placed on a large aluminum tool plate as shown in Figure 85(a). The tool plate was 0.5 inches thick and was used to create a flat surface which the laminate is allowed to conform to. Teflon film was placed on the tool plate to create a separation medium to ensure that the laminate does not adhere to the tool plate as shown in Figure 85(a). In addition to the Teflon, shims were placed all around the perimeter of the laminate and a thermocouple sensor was placed 0.5 inches away from the laminate as shown in Figure 85(a). Shims measuring 0.04 inches were placed all around the laminate so that thickness and volume fraction of the laminate may be controlled. Shims supported the weight of a large aluminum caul plate (which is wrapped in Teflon) which was placed on top of the laminate as shown in Figure 85(a) and Figure $85(b)$. The shims created a gap between the tool plate and the caul plate so that the final thickness of the laminate did not exceed that of the shims. Shim thickness of 0.04 inches corresponded to 0.005 inches/cured ply as recommended by the manufacturer. Thermocouple sensors were used to monitor the temperature of the laminate during the cure cycle. A layer of 
porous Teflon was placed on top of the caul plate and tool plate as shown in Figure 85(b). Porous Teflon was used to limit excess resin bleed from adhesion to surrounding surfaces. In addition to limiting adhesion, porous Teflon was used as it did not hinder air flow that was caused by vacuum force. A layer of 0.25 inch breather cloth was added on top of all components and sealant tape was placed all around the perimeter of the tool plate as shown in Figure 85(c). Breather cloth acted as a separating medium between the vacuum bag and all other components; allowing air flow while vacuum is applied. A vacuum bag was placed and an air tight seal was created with the sealant tape all around the perimeter of the tool plate. A pressure valve was placed by creating a small incision in the bag as shown in Figure $85(d)$. The packaged laminate was kept under 30 PSI of pressure by attaching a pump to the mushroom valve until placed into the autoclave oven where the curing process was carried out and pressure within the autoclave oven reached that of 85 PSI. 


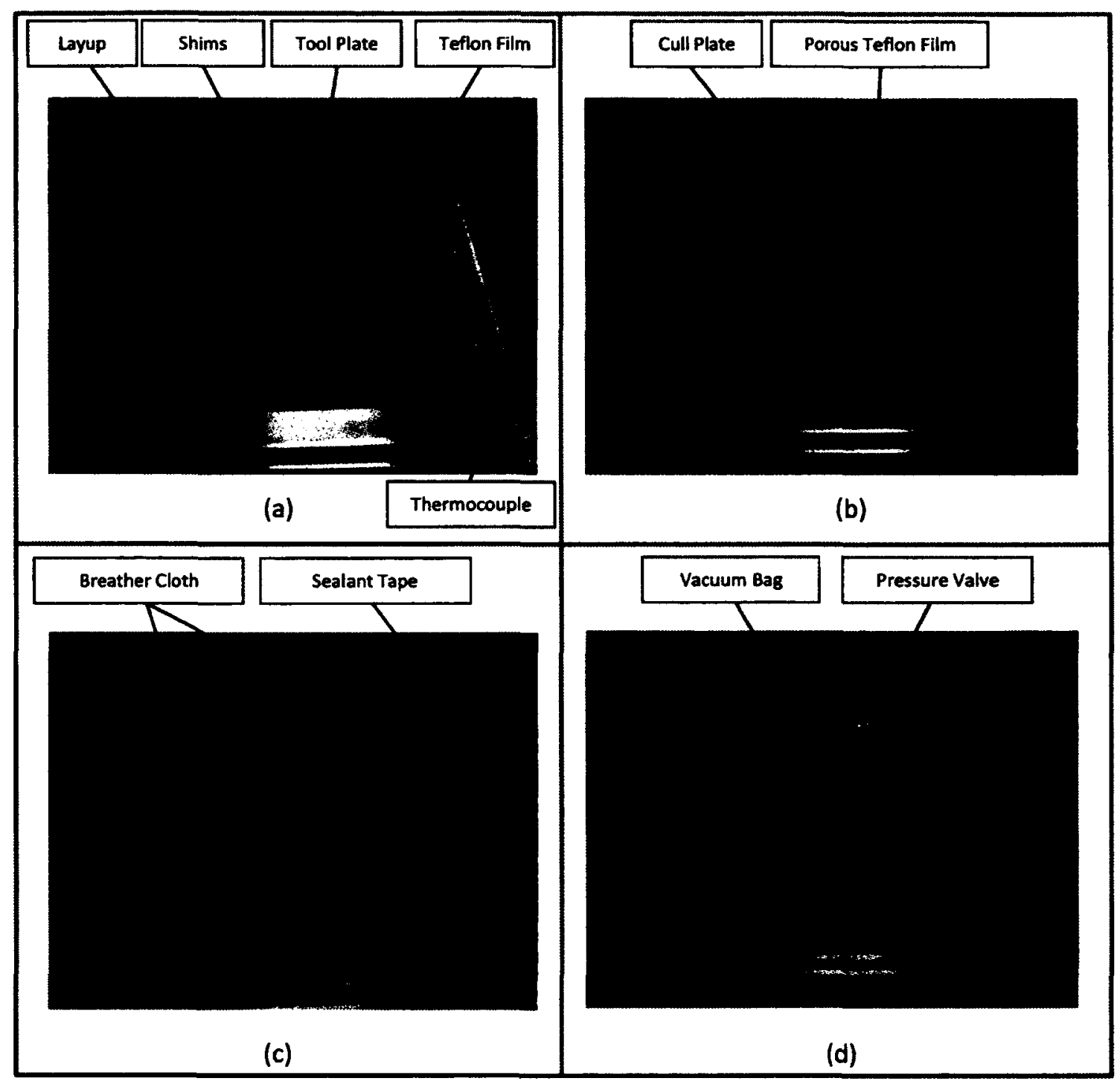

Figure 85: Layup Bagging: (a) Laminate Placement, (b) Covering of Laminate, (c) Breather Cloth, and (d) Pulled Vacuum

\subsection{Laminate curing}

Curing of the carbon fibre laminates was done in an autoclave oven according to the temperature and pressure profiles shown in Figure 86 which is recommended by the manufacturer. Ensuring cure temperatures was done through the use of three thermocouples. One thermocouple was placed on the bottom of the tool plate, one placed on top of the caul plate and one placed within the bag. 


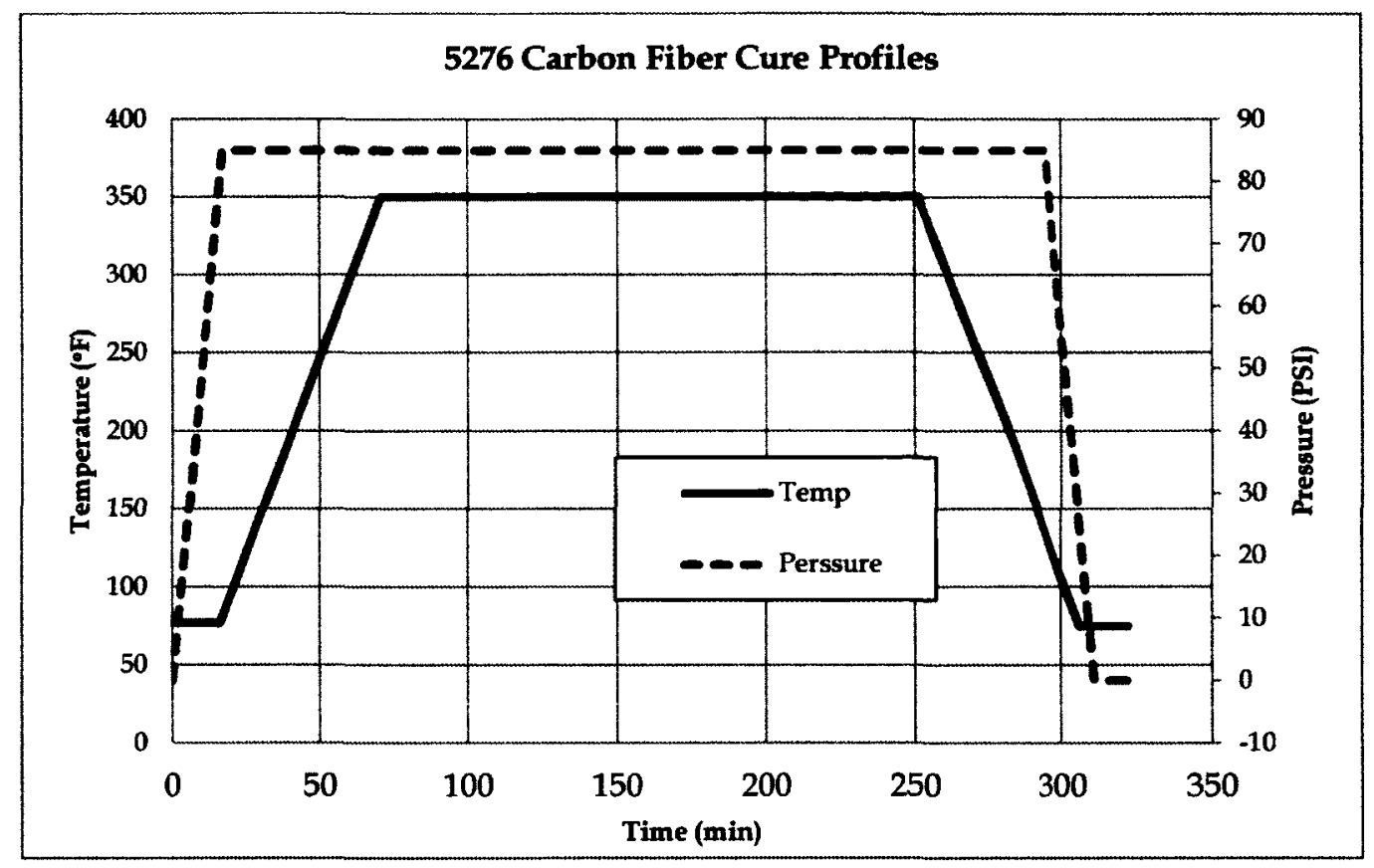

Figure 86: CYCOM 5276-1 Temperature and Pressure Profiles

\subsection{Laminate manufacturing results}

Inspections of the cured laminate revealed resin bleed all around the perimeter and a glossy surface finish on both sides of the laminate as shown in Figure 87. Both observations indicate that the laminate is neither resin starved nor rich. Laminate thickness measurements were taken at multiple locations, 3.0 inches away from neighbouring measurement locations along the laminate as shown in Figure 88. Measurements revealed that maximum variation of thickness between any given points was limited to 0.004 inches. During removal of the vacuum bag, noticeable lack of laminate flatness was observed as shown in Figure 87 . The lack of flatness is attributed to the formation of bi-phase laminates. A bi-phase laminate is a laminate which has two possible low stress states; both of which result in lower amount of internal residual stresses. Much care was taken to avoid this; however, bi-phase laminates were the result of misalignment of the $\pm 45^{\circ}$ plies; as the angles varied even by the smallest amount, residual stressed occurred and caused this phenomenon to occur. After cutting the laminate 
into the smaller substrate, the effect of the bi-phase laminate was minimized so that it was unnoticeable.

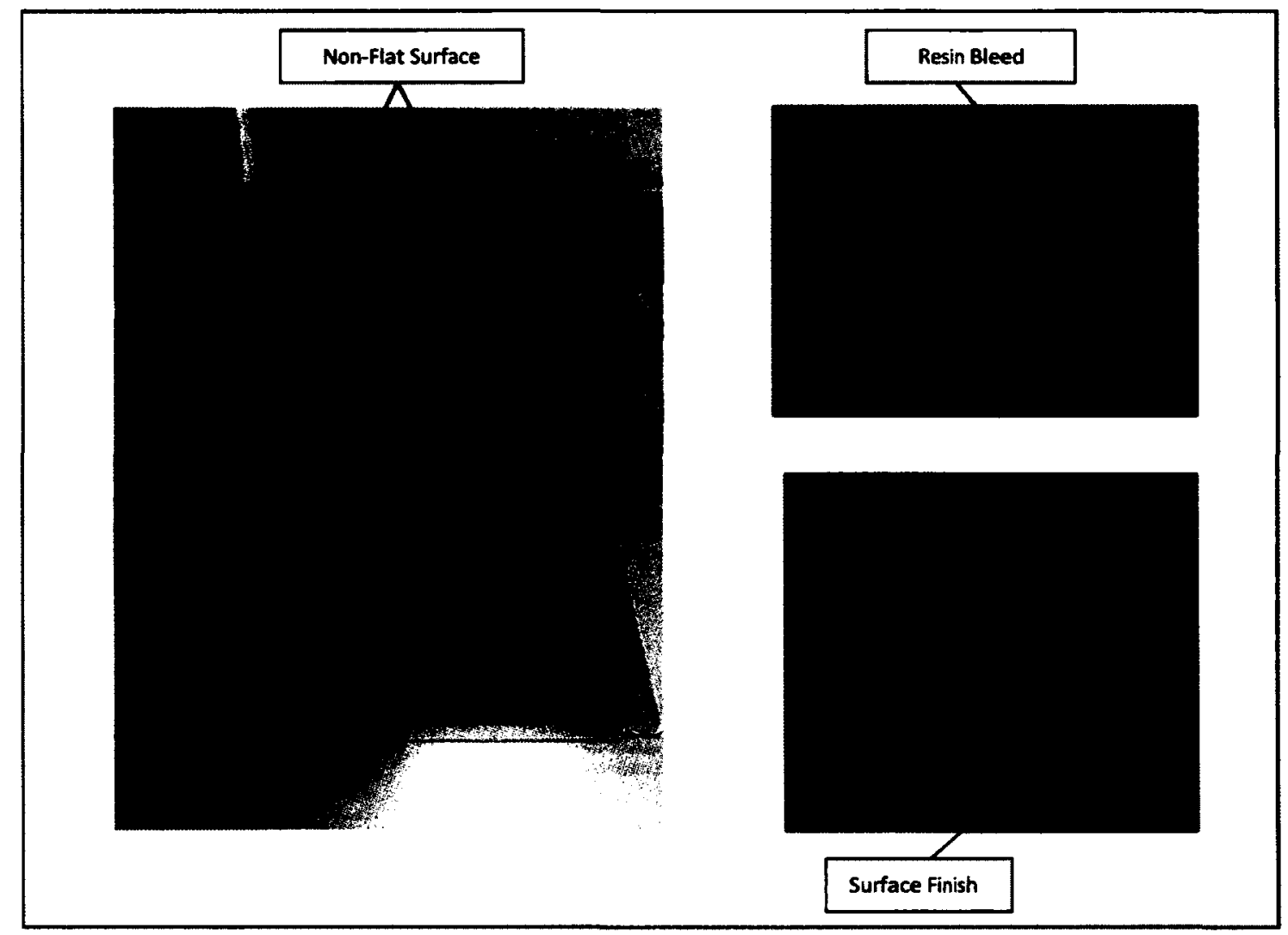

Figure 87: As Manufactured Carbon Fibre Laminates

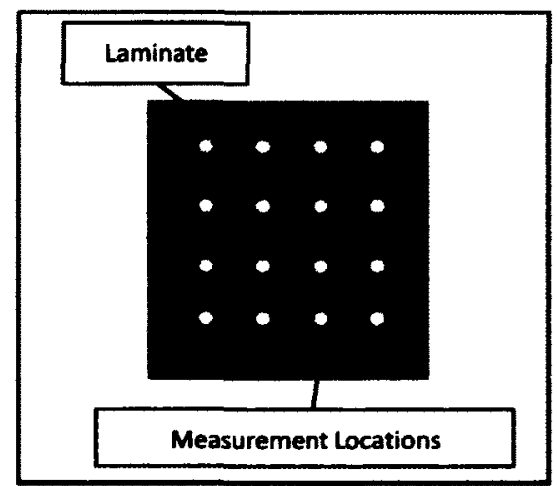

Figure 88: Laminate Thickness Measurements 


\subsubsection{Patch manufacturing}

The manufacturing procedure used for the patches is very similar to that which was used for manufacturing of the substrates. However, unlike the substrate, the patches had a different layup: $[0,0,45,-45]_{s}$.

The Specialty Materials $5521 \mathrm{~F}$ boron prepreg tape is only 6.0 inches wide and therefore cutting plies that are at $\pm 45^{\circ}$ did not achieve large enough laminates that could produce two or more patches. Instead of large laminates, laminates measuring $5.5 \times 2.5$ inches were created following the same procedure as in section 3.1.3.4.1. These laminates were made larger by 0.25 inches all around the perimeter as shown in Figure 89. The manufactured laminates for the substrate had a minimum of 0.5 inches all around the substrate so that edge effect could be minimized. For these laminates, 0.25 inches was an adequate length as the surface area of the patch was much smaller. Smaller patch surface area resulted in resin bleed that was considerably less than that of the substrate laminates.

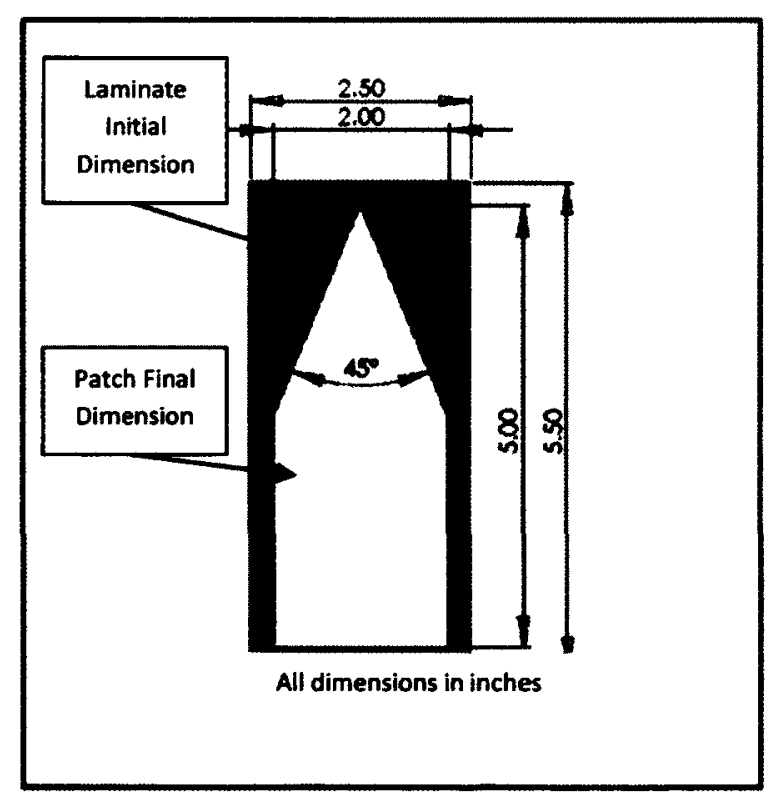

Figure 89: Patch and Laminate Geometry 
The patches were manufactured from $5.5 \times 2.5$ inch rectangles with the above mentioned layup. These rectangles were cut down to design size as per Figure 89.

To manufacture the patches, 8 plies were cut for each laminate, 4 plies were cut at $0^{\circ}, 1$ at $+45^{\circ}$ and 3 at $-45^{\circ}$. Alignment of each ply as well as entrapped air removal was done following the same procedure used for the manufacturing of the substrate; details may be found in section

\subsubsection{1.}

\subsection{Patch bagging (Trial 1)}

Bagging of the patches was done in the same manner as that which was used for the substrate manufacturing, with the exception of the number of patches placed in a single vacuum bag assembly. There were 6 patches placed underneath, each caul plate with shims surrounding the perimeter as shown in Figure 90 . Shims provide a 0.048 inch clearance between the tool plate and the caul plate (0.006 inch for each cured ply) so that the final thickness of the patches is not lower than 0.048 inches. 


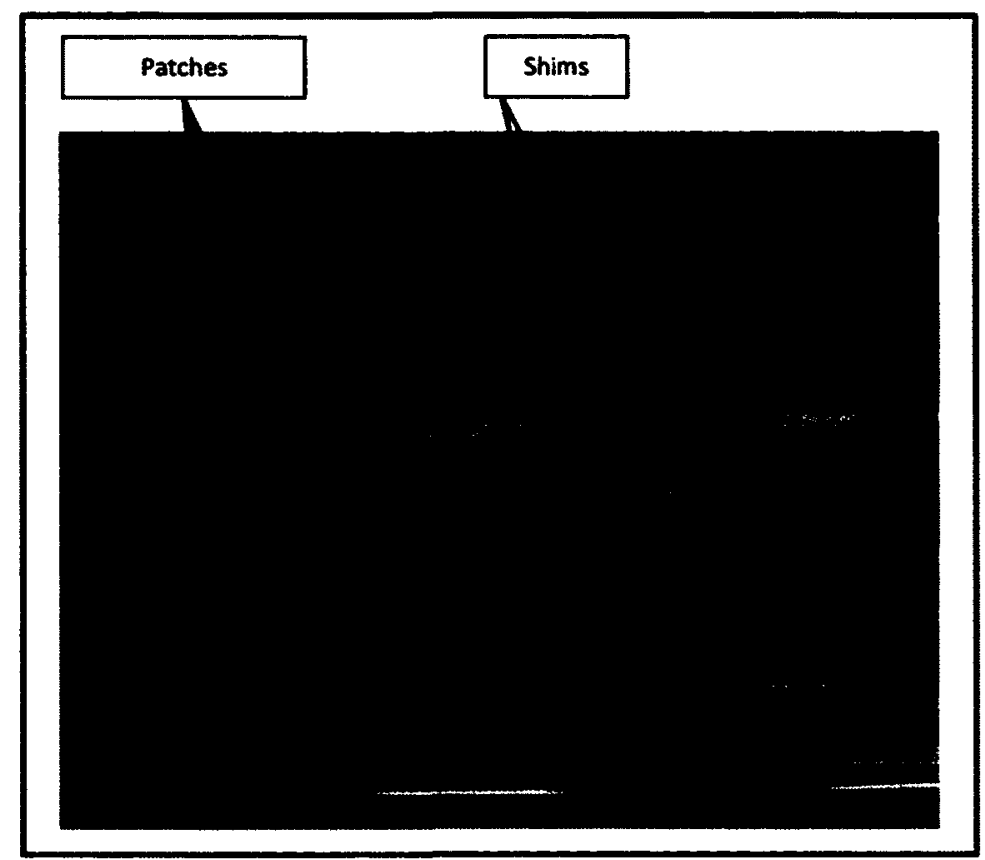

Figure 90: Patch Bagging

\subsection{Patch curing (Trial 1)}

Curing of the patches was done within an autoclave oven according to the procedure that is recommended by the manufacturer; cure temperatures as well as pressure profiles are shown in Figure 91. Ensuring cure temperatures was done through the use of three thermocouples. One thermocouple was placed on the bottom of the tool plate, one placed on top of the caul plate and one placed within the vacuum bag. 


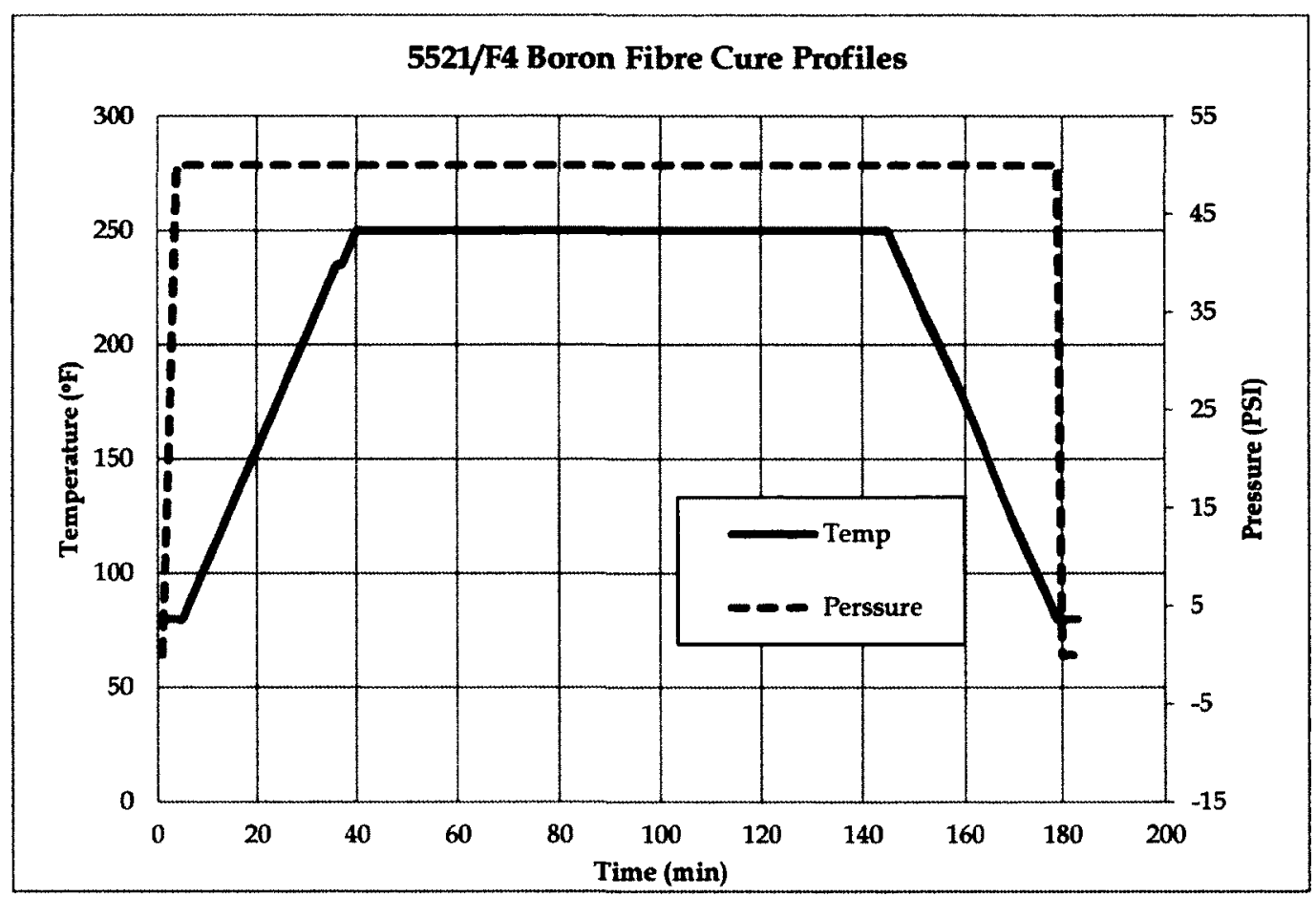

Figure 91: Specialty Materials 5521F Boron Prepreg Tape Temperature and Pressure Profiles

\subsection{Patch manufacturing results (Trial 1)}

Inspection of the laminates revealed fibre distortion, as shown in Figure 92. Distortion of the patches was attributed to the separation of fibres within the top and bottom $0^{\circ}$ ply. It was determined that fibre displacement was caused by either too much pressure imposed by the caul plates or the uncontrolled resin flow; both of which are valid reasons and may have played a role. For these two reasons, it was determined that another method of manufacturing would have to be tested and used to manufacture the patches. 


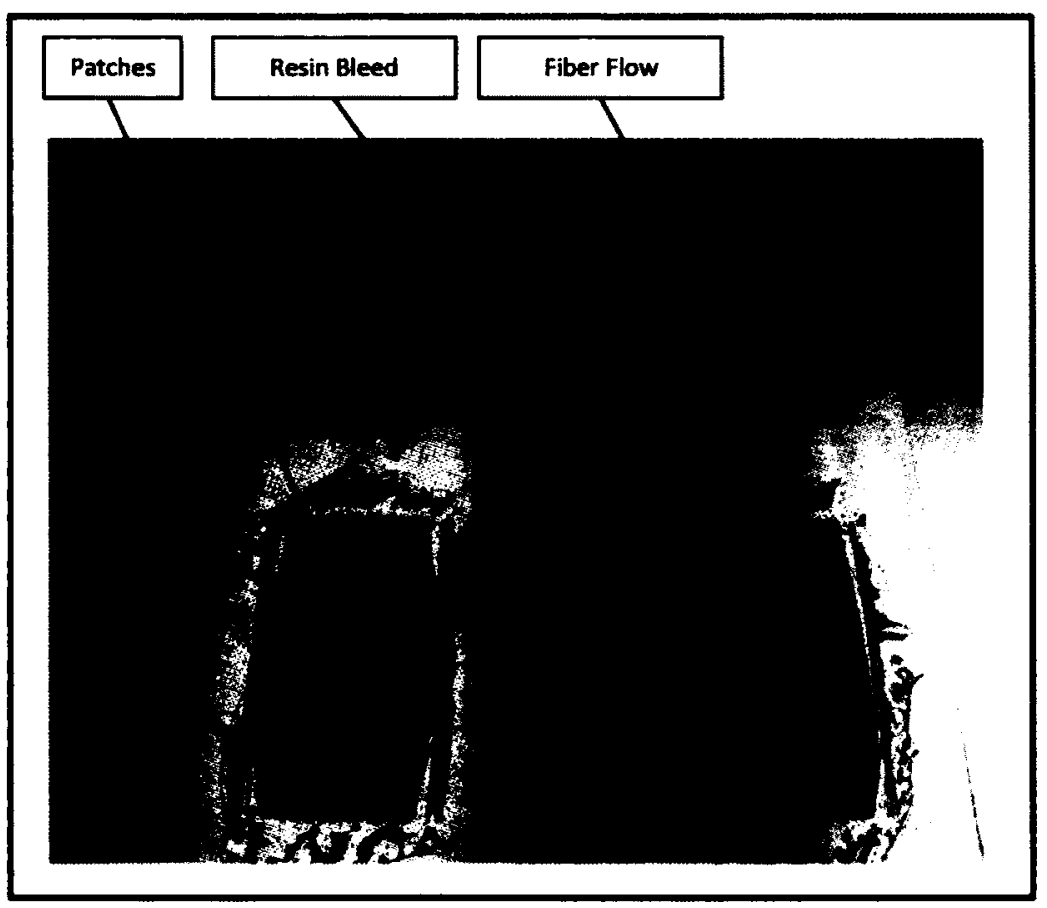

Figure 92: Boron Patch Distortion

\subsection{Patch manufacturing (Trial 2)}

Two methods of fibre retention were explored in attempt to minimize fibre displacement. The first method involved wrapping the outer edges of the patch with tape while the second involved the addition of a peel ply layer on the top and the bottom of the laminate; both are shown in Figure 93. The addition of the tape was done in such a manner that it would only contact 0.25 inches all around the perimeter; which would be removed during machining. A series of 20 small punctures were made along the long side of the patch and 10 along the short side. These punctures were created to promote resin flow. 


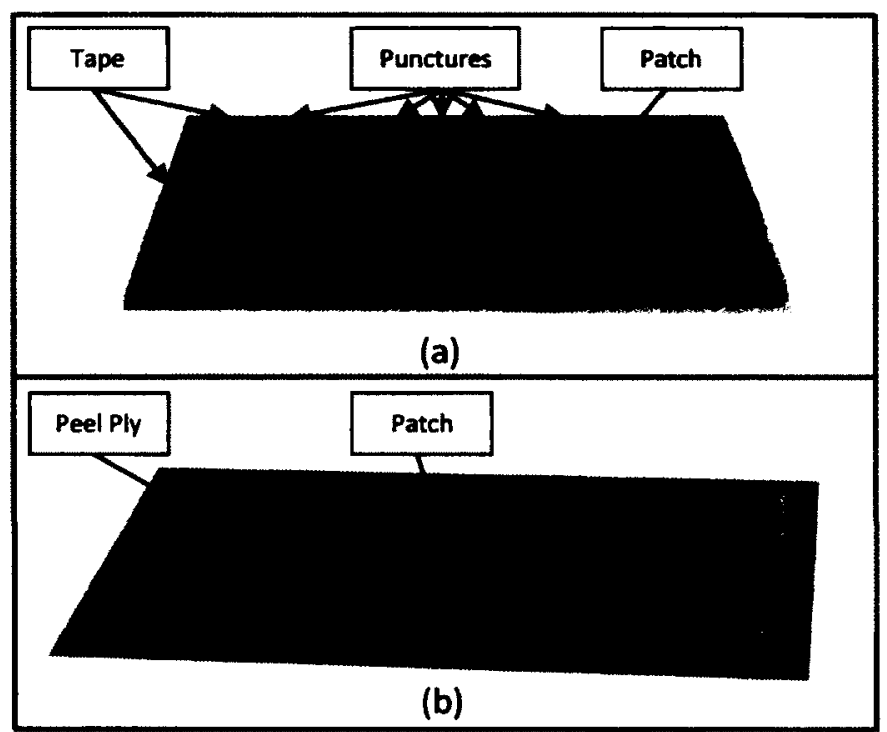

Figure 93: Patch Manufacturing Trial 2: (a) Patches with Tape Edge Dams, (b) Patches with Surrounding Peel Ply

\subsection{Patch bagging (Trial 2)}

Bagging was done in the same manner as the bagging which is described in section 3.1.3.4.2; however, the alignment of shims as well as their sizes was changed to accommodate the new trial. Two methods of shim placements were attempted; the first was the use of shims all around the perimeter of the patch (SP-1) while the second was the placement of shims all around the caul plate (SP-2) as shown in Figure 94. SP-1 served as both edge damns for the resin flow as well as ensuring that there is no warping of the caul plate while under pressure. SP-2 was that which was used in trial 1 . The final shim thickness for patches with tape remained the same as 0.048 inches. Shim thickness for peel ply wrapped patches was changed to 0.060 inches to accommodate for the addition of 0.012 inch thickness of the two layers of peel ply. 


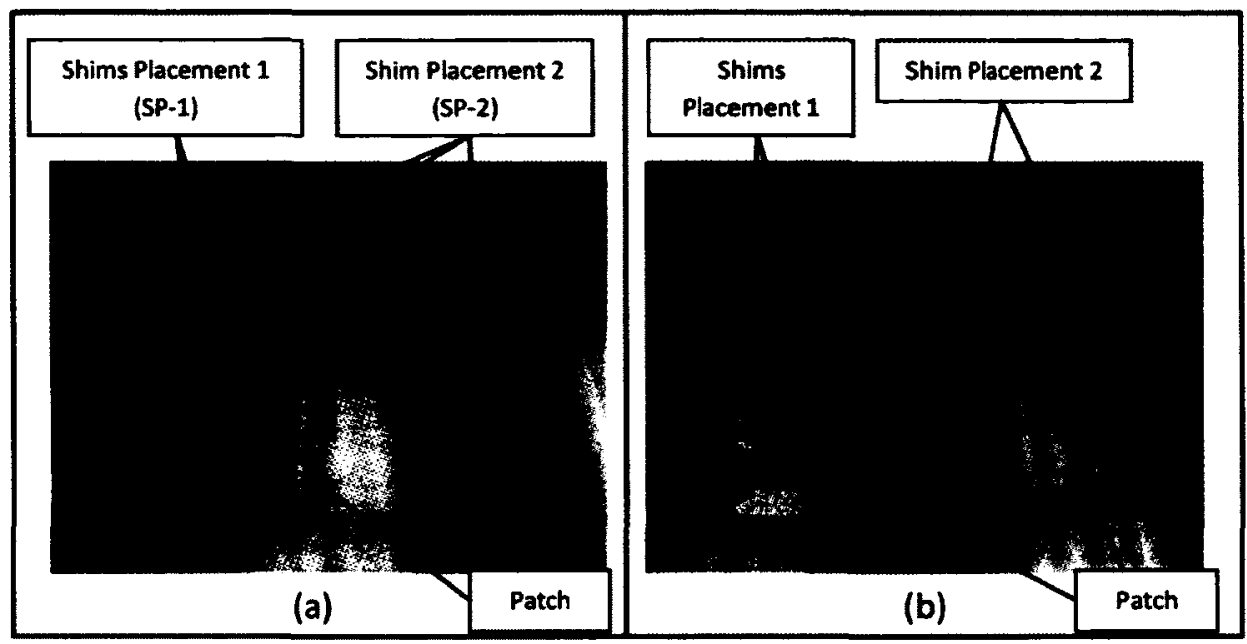

Figure 94: Patch Bagging Trial 2: (a) Patches with Tape Edge Dams, (b) Patches with Surrounding Peel Ply

\subsection{Patch curing (Trial 2)}

Curing of the patches for trial 2 was carried out exactly as indicated in section 3.1.3.5.2.

\subsection{Patch results (Trial 2)}

All variations of patch trial 2 showed good fibre alignment along the top and bottom $0^{\circ}$ plies; $\pm 45^{\circ}$ plies could not be inspected as they were in between the $0^{\circ}$ plies. Some patches yielded better resin flow and consequently better surface finish. Figure 95(a) shows results of the patch with tape edge dams and surrounding shims. Evidence from the figure indicated that there was little resin bleed from the main patch, consequently the resin remained and formed ridges along the top surface of the patch. Figure $95(b)$ shows the patch with tape edge dams and no surrounding shims. This patch shows good resin bleed and surface finish. Figure 95(c) shows results of the patch with peel ply and surrounding shims, the entire patch was in good condition but there were minor flaws that were the result of a tear within the peel ply. Figure 95(d) shows results of the patch with peel ply and no surrounding shims; the entire patch had good surface finish as well as resin bleed. 
Of all four patch variations, both taped edges and surrounding peel ply with SP- 2 yielded better surface finish and resin bleed as compared to patches utilizing SP-1. In anticipation of bonding and its associated surface preparation for sensor instrumentation, the use of peel ply with no surrounding shims was selected as the manufacturing method of choice. The use of peel ply created a rough surface for sensors to adhere to as well as maintained a clean surface until bonding procedures were carried out and peel ply layers removed.

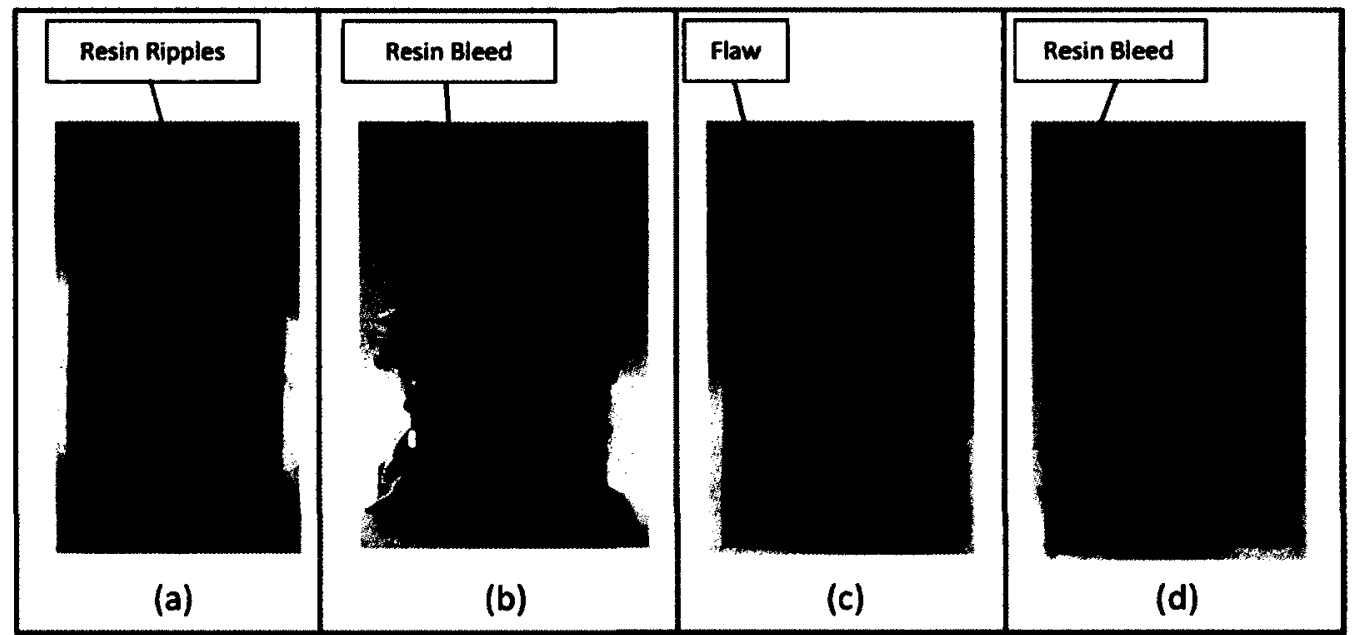

Figure 95: Patch Results Trial 2: (a) Patch with Tape Edge Dams and Surrounding Shims, (b) Patch with Tape Edge Dams and No Surrounding Shims, (c) Patch with Surrounding Peel Ply and Surrounding Shims and (d) Patch with Surrounding Peel Ply and No Surrounding Shims

\subsubsection{Patch to substrate bonding}

This section describes the processes that were taken to bond the manufactured patches to the manufactured substrates.

\subsection{Bonding setup}

Bonding of patches to the substrate was started by surface preparation of the substrate; this was achieved by grit blasting the surface of the substrate where the patch was to be bonded as shown in Figure 96(a). A single layer of FM73 film adhesive was cut to the size of the patch and 
attached to one side of the patch as shown in Figure 96(a). Exact alignment of the patch to the substrate was achieved by using a right angle ruler to maintain edge alignment as shown in Figure 96(a) and Figure $96(\mathrm{~b})$. The ruler served two purposes, the first was to ensure that both the edges of the patch as well as the edge of the substrate coincide and the second was to ensure that the patch was in the correct location along the substrate. Alignment of the patch to the substrate is demonstrated in Figure 96(b). The final step in preparation of the coupons was to remove the entrapped air from between the patch and the FM73 adhesive layer as well as between the substrate and the FM73; this was achieved by debulking the coupons. Because there existed a patch on either side of the substrate, tracks were utilized to support areas where no patches existed; this is shown in Figure 96(c). This was done to ensure no introduction of curvature within the coupons. The debulking procedure is summarized in Table 5.

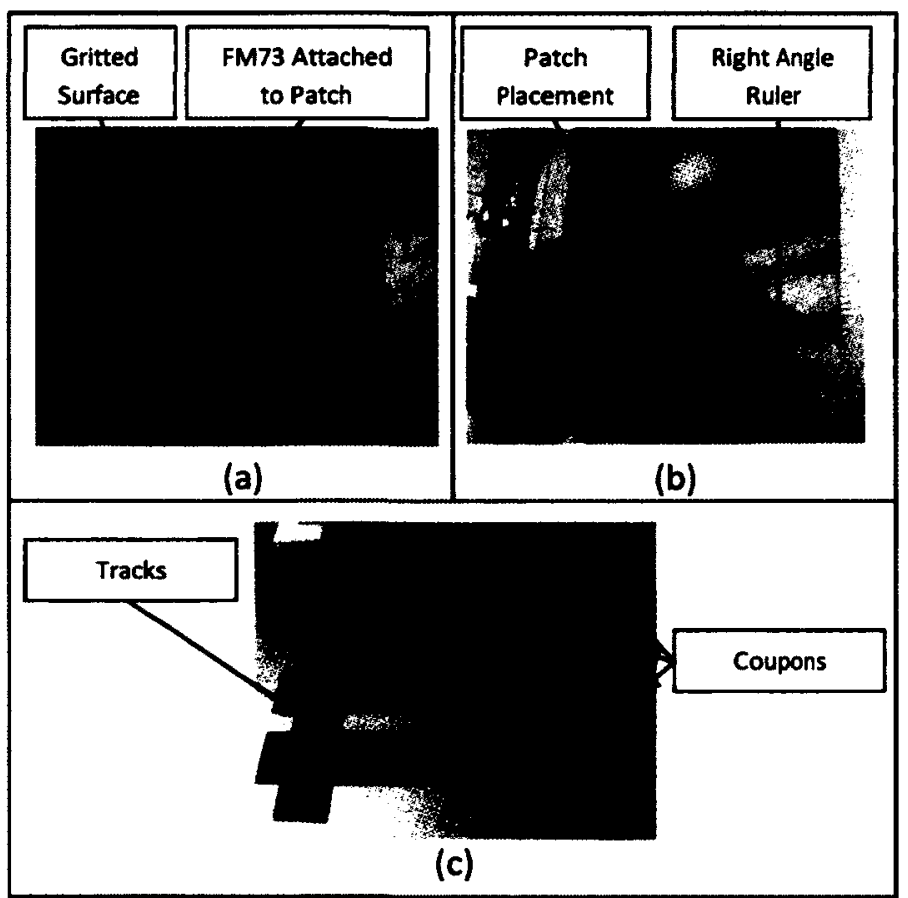

Figure 96: Bonding Procedure: (a) Surface Preparation, (b) Patch to Substrate Alignment and (c) Entrapped Air Removal 
Table 5: Bonding Debulking Procedure

\begin{tabular}{|l|l|}
\hline \multicolumn{1}{|c|}{ Preasure } & \multicolumn{1}{|c|}{ Time } \\
\hline 5 PSI & 5 minutes \\
\hline $10 \mathrm{PSI}$ & 5 minutes \\
\hline $15 \mathrm{PSI}$ & 5 minutes \\
\hline $20 \mathrm{PSI}$ & 5 minutes \\
\hline $25 \mathrm{PSI}$ & 5 minutes or more \\
\hline
\end{tabular}

\subsection{Coupon (final assembly) bagging}

Due to the presence of a patch on either side of the coupon, the traditional bagging method could not be applied; instead, an envelope bagging method was implemented. Envelope bagging involves wrapping the coupon in the vacuum bag; this method allows all around pressure distribution on the coupon.

In preparation of bagging, each coupon was individually wrapped with porous Teflon film as shown in Figure 97(a). Each coupon was then placed onto the bag and separated with breather cloth as shown in Figure 97(b); breather cloth was used so that a path for air is created such that vacuum may be evenly applied to the coupon. Once sealed using sealant tape, vacuum was pulled and pressure was applied on both sides of the coupon as shown in Figure $97(\mathrm{c})$. 


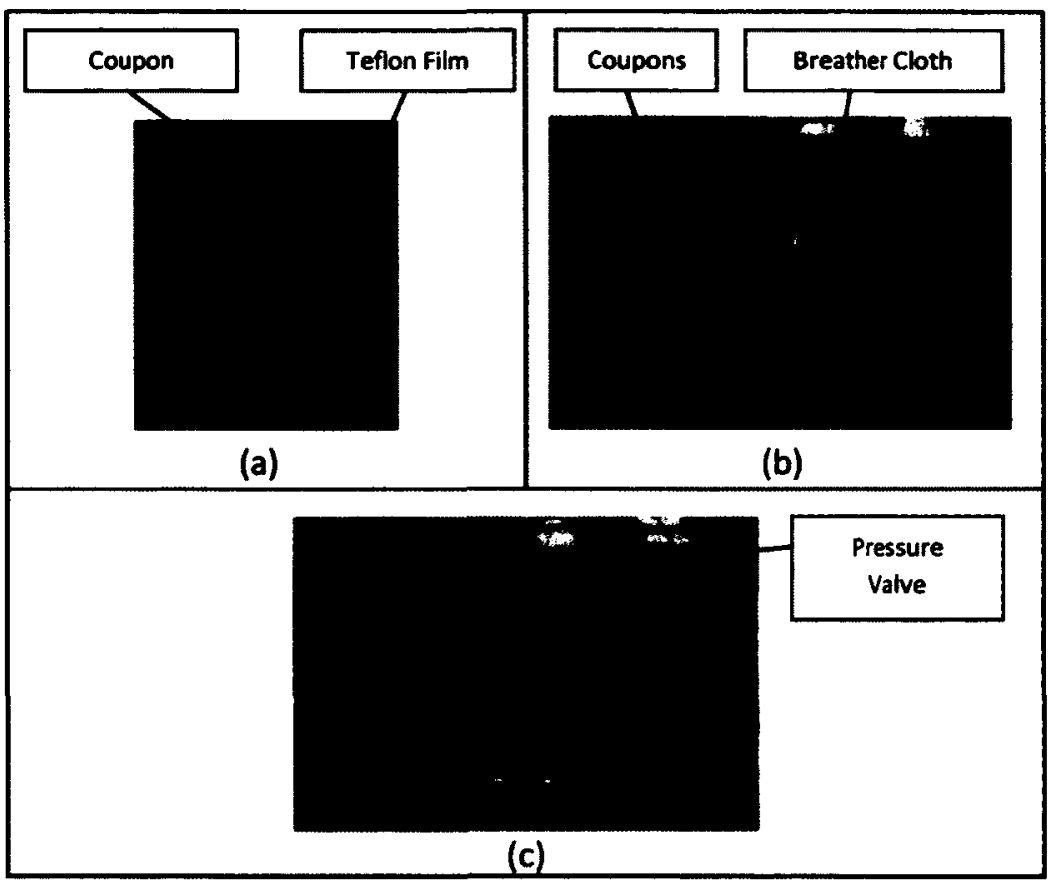

Figure 97: Bonding Bagging: (a) Coupon Wrapping, (b) Coupon Placement (c) Applying Vacuum

\subsection{Bonding curing}

Curing of the adhesive was done in accordance to the manufacturer's pressure and temperature profiles shown in Figure 98.

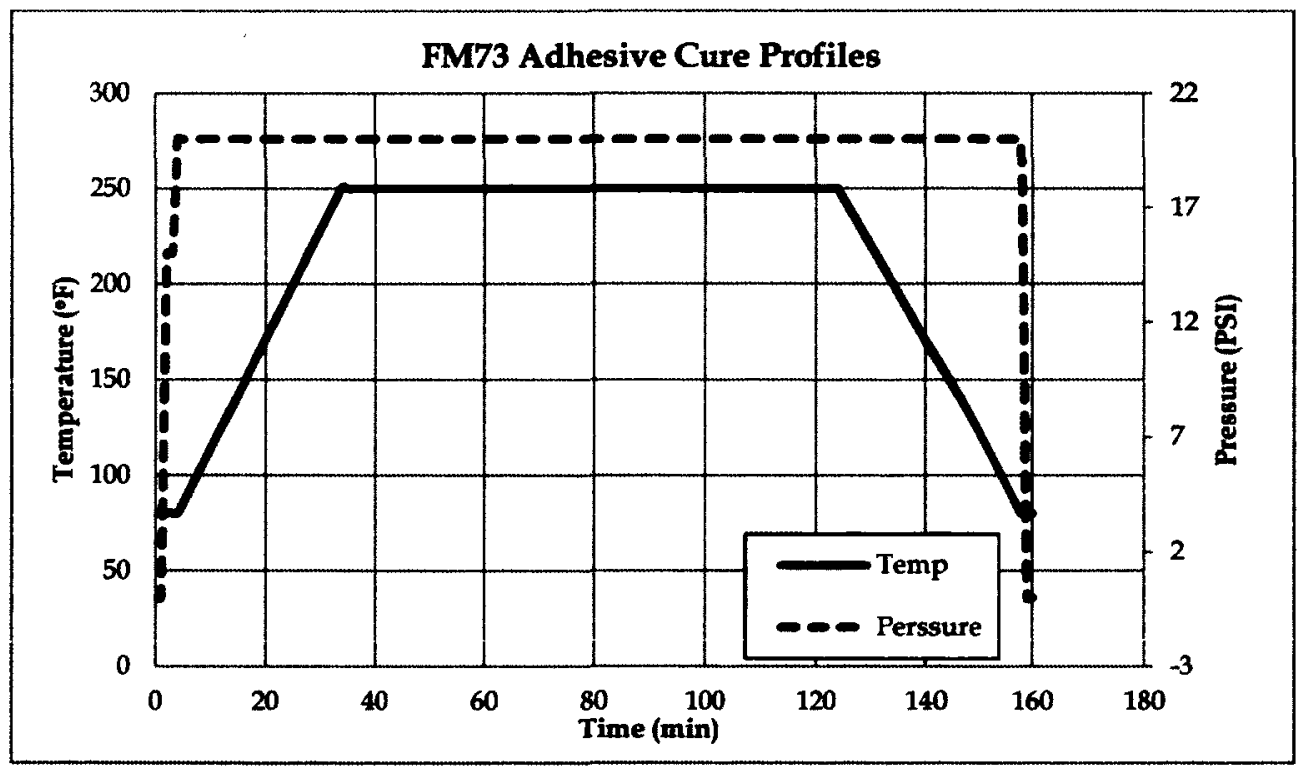

Figure 98: Cytec FM73 Film Adhesive Cure Profile 


\subsection{Bonding results}

Investigation revealed good bleed of FM73 all around the bonded patch as shown in Figure 99, which indicates good bonding. However, visual inspection must be accompanied by a certified NDE technique that would ensure full adhesion between the patch and the substrate. An ultrasound C-scan inspection was utilized on all the coupons, results of which are shown in Figure 100. Images found in Figure 100 show patches with varying color scale. The color blue within the scale shown in Figure $100(a)$ indicates that $0 \%$ of the transmitted ultrasound waves were received by the sensors, thus indicating a poor bond. Pink within the same scale represents $100 \%$ of the transmitted signals were received by the sensor, thus indicating a strong bond. Figure 100 shows 8 patches; Figure 100(b) shows 4 patches that have poor adhesion while Figure 100 (c) shows patches that have good adhesion. Of all manufactured coupons, 4 revealed signs of inadequate adhesion. On all scanned patches, there exists a small portion of the patch that exhibits an inconsistent bond, this is found at the tip and can be seen in Figure 100 (c). This inconsistency may be attributed to lack of bonding or the rapid change of geometry which may have caused a change of acoustic signal propagation and reflection. The inconsistency may have played a role in the disbond; however, it may have caused disbond to occur at an earlier stage of the coupon life cycle. This was not a concern as the SHM systems were used to determine whether they could detect the disbond, regardless of when they occurred. 


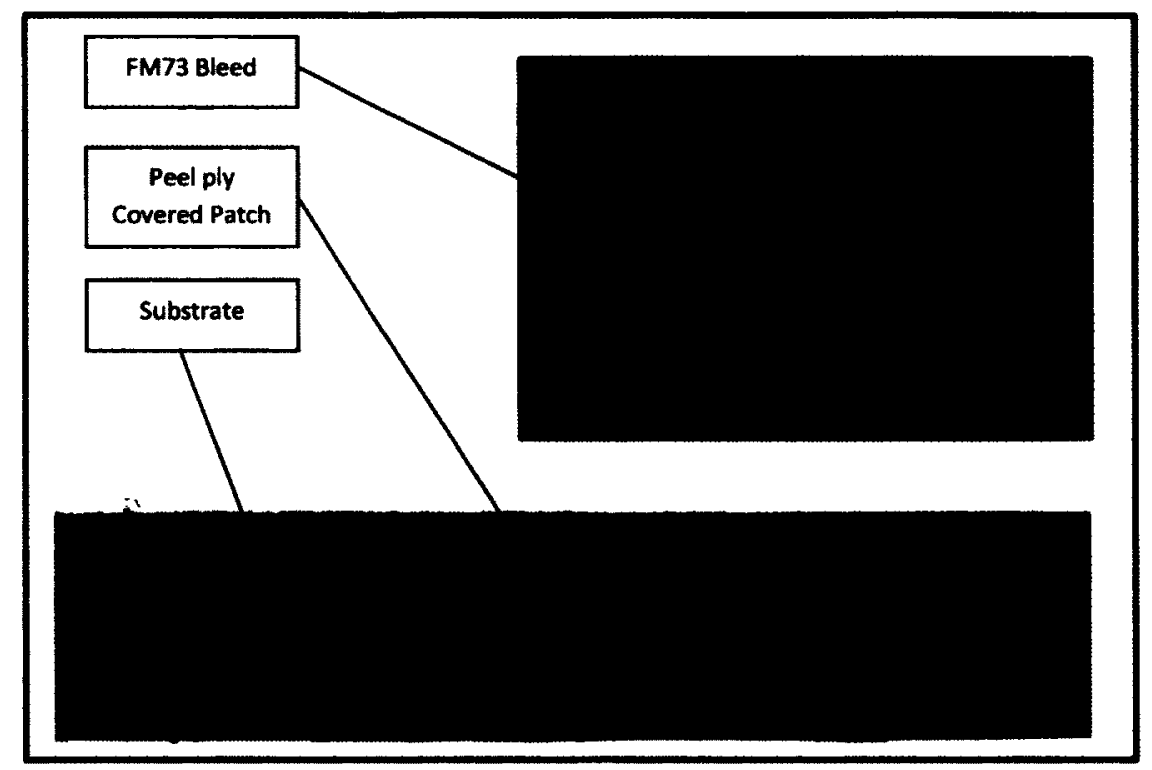

Figure 99: As Manufactured Coupon

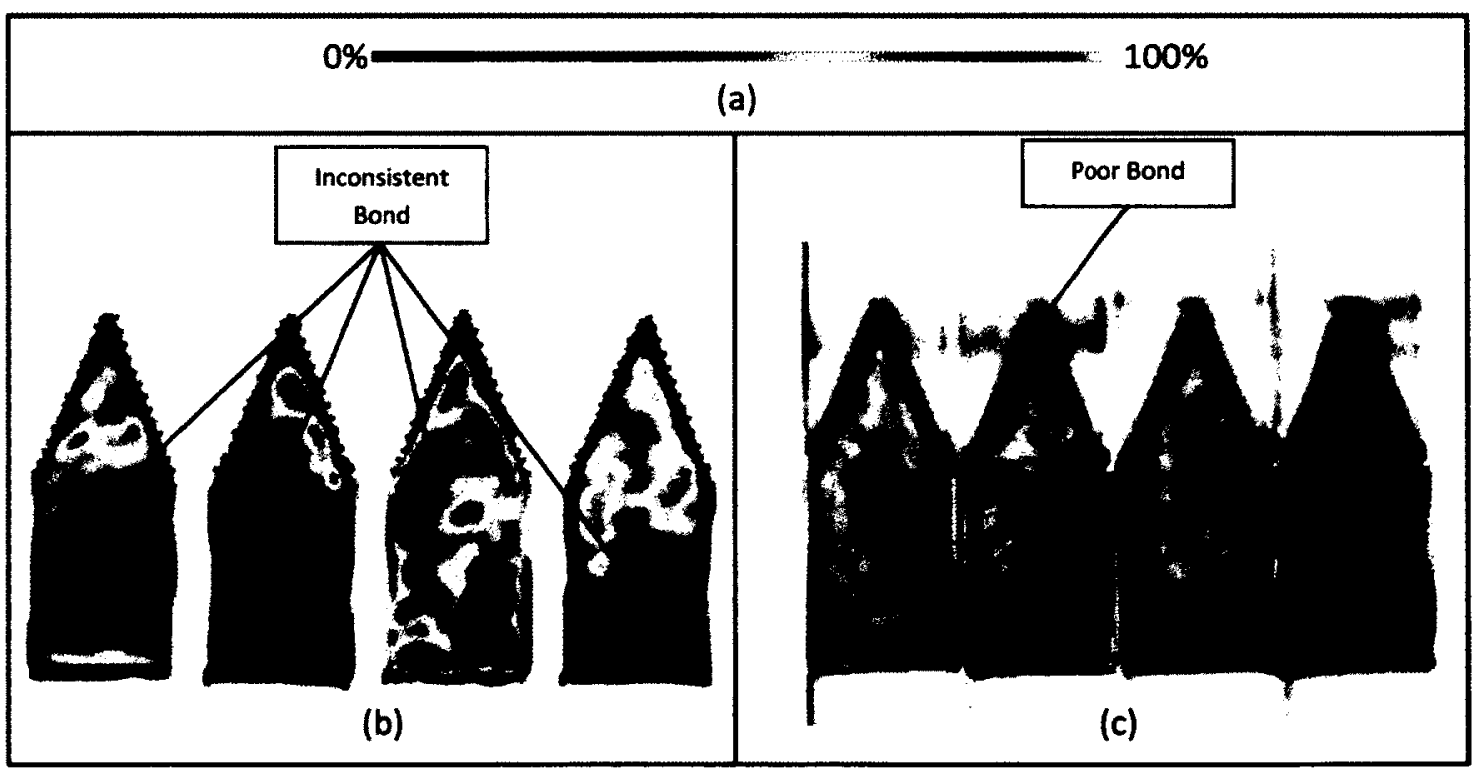

Figure 100: Coupon Ultrasound C-Scan Inspections

\subsubsection{Coupon instrumentation}

Three types of SHM systems were evaluated within this study: Acellent (an Acousto-Ultrasonic system), Capacitance Disbond Detection Technique (CDDT) and Surface Mountable Crack Detection System (SMCDS). All three systems utilize different sensors and acquisition systems. Each coupon was instrumented on both sides using two different SHM sensors. Coupons were 
split into two groups, one group was instrumented using CDDT while the second group was instrumented using SMCDS. Both coupon groups were instrumented using the AcoustoUltrasonic system.

\subsection{Acousto-Ultrasonic sensors}

The Acousto-Ultrasonic (AU) system used piezoelectric elements as shown in Figure 101, which acted as both sensors and actuators. Piezoelectric elements required proper adhesion to the structure for maximum mechanical wave transmittance. In addition to proper adhesion, sensor location was important; it defined the area to be monitored. Adhesion of sensors to the coupon was done similar to that of strain gauge instrumentation. Surface preparation of the coupon was carried out in the following steps: 1 . surface cleaning using alcohol, 2 . Surface sanding using 400 grit sand paper, 3. surface cleaning using alcohol and 4. surface neutralization. Step 2 was done so that the surface of the substrate was no longer glossy and is rough, while step 4 was done so that chemicals used during the cleaning process are neutralized to a $\mathrm{pH}$ of 7 . The four steps used in the surface preparation process ensured strong adhesion, as the surface was rough and contaminate-free. A similar process was used for the preparation of the sensors; however, the sensors were not sanded.

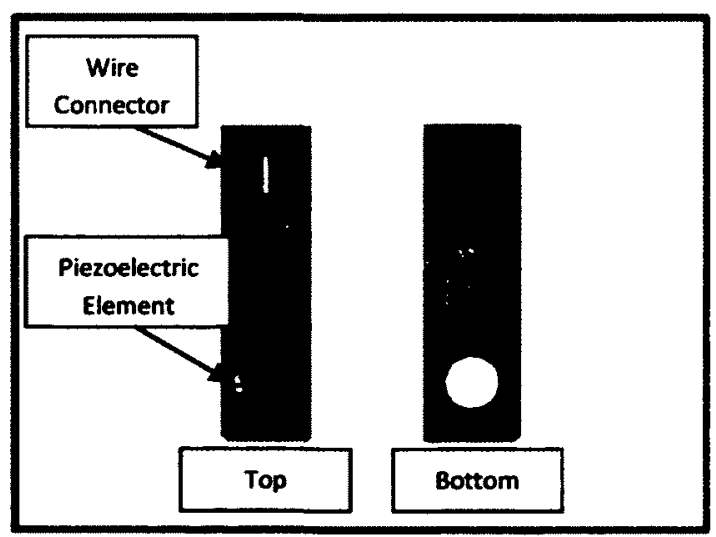

Figure 101: Acellent Piezoelectric Sensors 
Instrumentation using the piezoelectric elements was done both on the patch and on the substrate using M-Bond AE-10 adhesive as shown in Figure 102 and Figure 103. There exists two different sensor configurations as shown in Figure 102(a) and Figure 102(b). Sensor layout 1 (Figure 102(a)) utilizes 8 sensors while sensor layout 2 (Figure 102(b)) utilizes 9 sensors. The addition of a single sensor to the layout was done to increase the scanning area and therefore the scanning accuracy. Both the area and accuracy were increased due to the addition of scanning paths as shown in Figure 102(c). Scanning paths are the shortest distances between elements in which waves propagate from one element to another. Changes in signals along that path indicate the presence of damage. Figure 103 shows a fully instrumented coupon using piezoelectric elements and sensor layout 2.

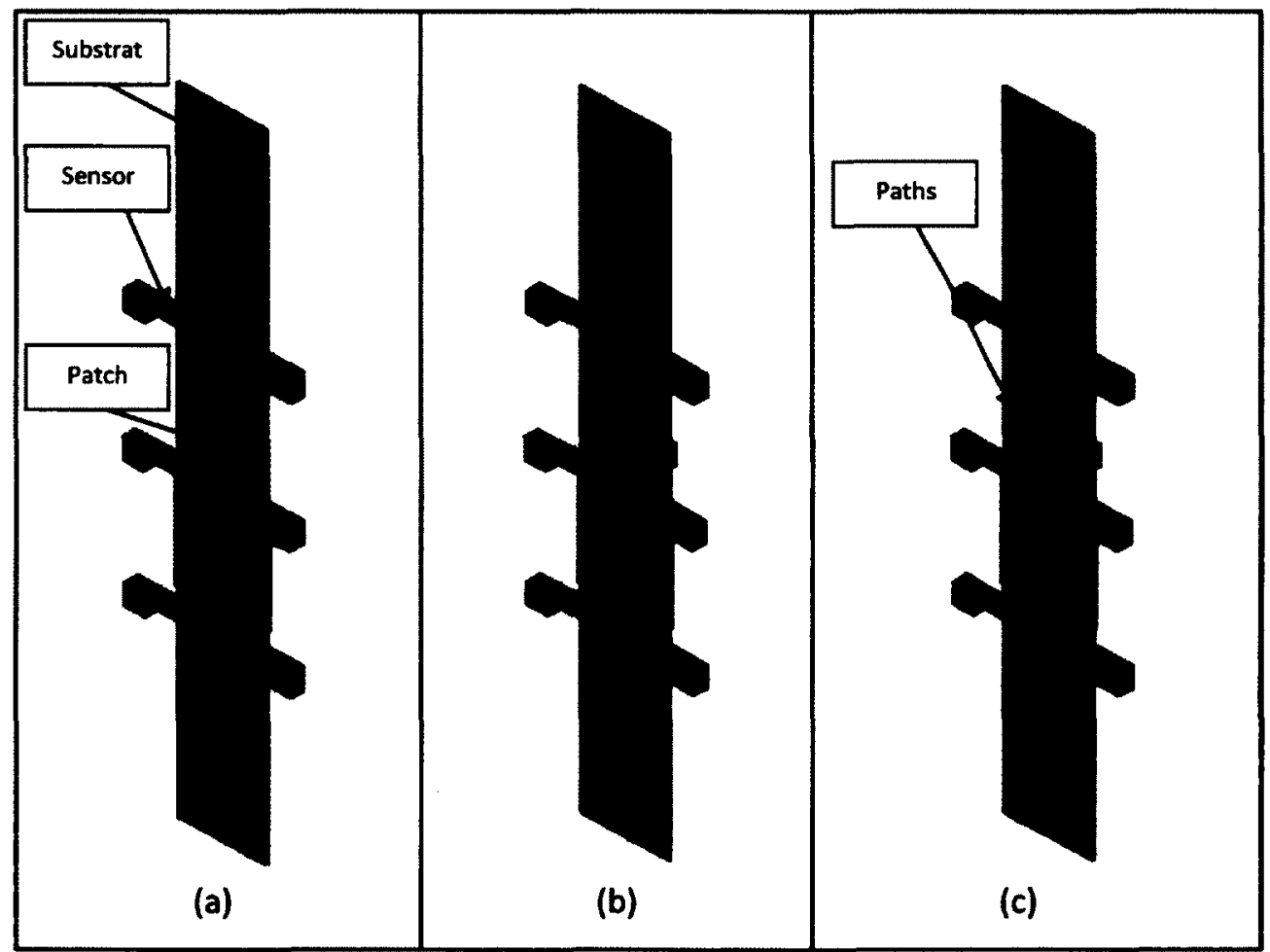

Figure 102: Piezoelectric Sensors Layout: (a) Sensor layout 1, (b) Sensor layout 2 and (c) Sensor scanning paths 


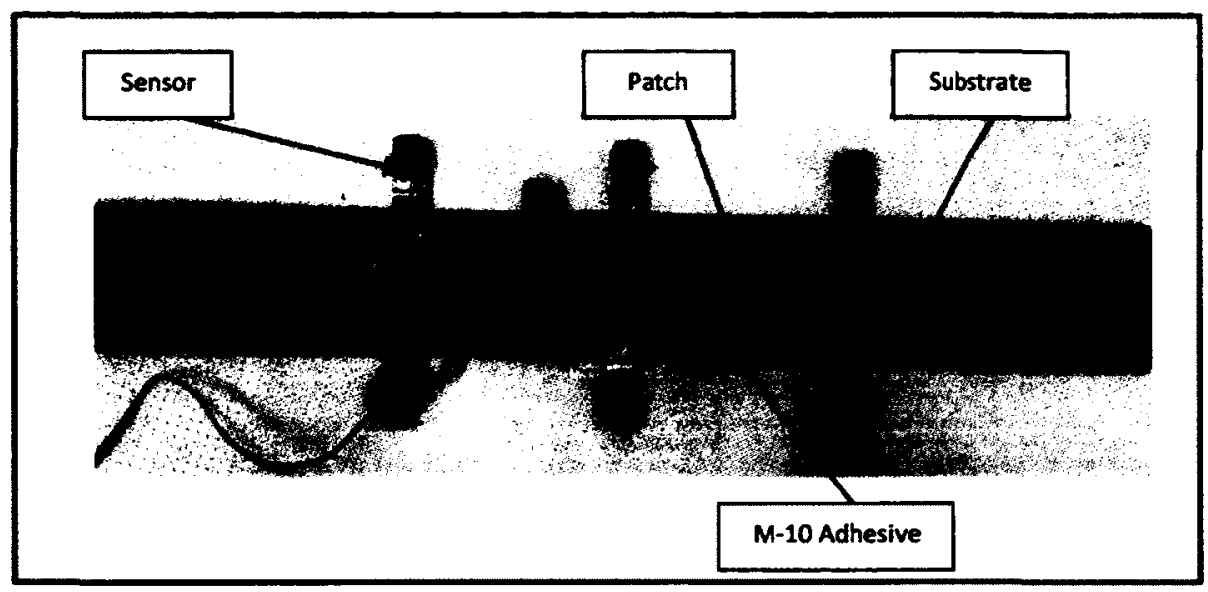

Figure 103: Instrumented Coupon

\subsection{Capacitance Disbond Detection Technique (CDDT) sensors}

CDDT is a technology in which, two adhesively joined conductive materials form a capacitor while the adhesive layer acts as the insulator between them. For this technology, there was no need for the installation of additional sensors as it utilizes electrical conduction properties of each material to form a capacitor. For these coupons, the carbon fibre substrates are conductive while both the boron fibre patches and the adhesive layer are non-conductive. To form a capacitor, there must exist two conductive layers with a non-conductive separation medium. The adhesive was used as the non-conductive medium, while the substrate and the patch are the conductive layer. Due to the low electrical conductive properties of boron, a high purity silver paint conductive layer was applied to the surface of the patch as shown in Figure 104(a). Wires were added from both the patch and the substrate as shown in Figure 104(b); and connected to a multi-meter as shown in Figure $104(c)$. The multi-meter was used to measure the capacitance of the system. 


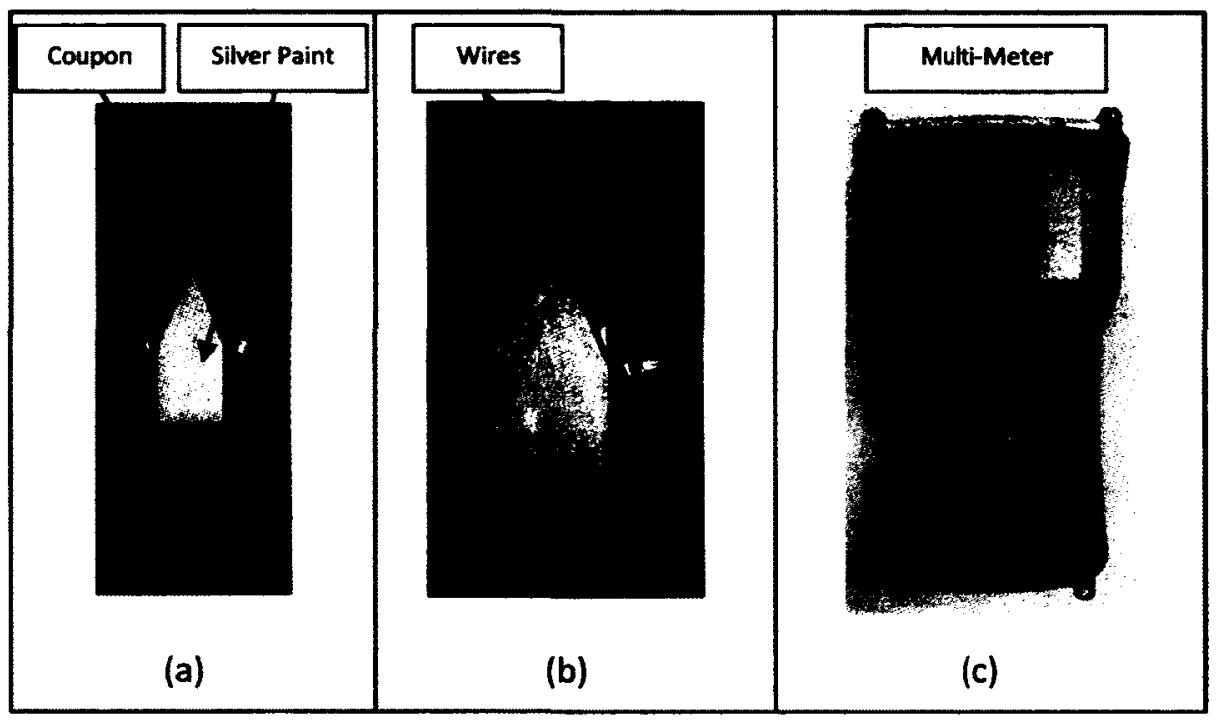

Figure 104: CDDT Sensor

\subsection{Surface Mountable Crack Detection System (SMCDS)}

SMCDS is a technology in which a thin conductive circuit is painted onto the surface of the specimen under evaluation. Growth of $\operatorname{crack}(s)$ through the Surface Mountable Crack Sensor (SMCS) breaks the circuit causing it to trip, indicating crack growth. SMCS are composed of three primary layers: an insulating layer, a conductive layer and a protective layer as shown in Figure 105. The insulating layer ensures little to no voltage transfer between the conductive layer and the conductive substrate.

Preparation of the substrate surface for the insulating layer was done in the same manner as described within section 3.1.3.7.1. Three layers of insulating paint were used to isolate the SMCS from the coupon. Each layer was applied and allowed to harden for $10 \mathrm{~min}$ prior to application of an additional layer as shown in Figure 105(a) and Figure 105(b). Application of the insulating layer was only done at the top point of the patch and substrate. Small thin lines of conductive silver paint were applied on the hardened insulating layer as shown in Figure 105(c) and Figure 105(d). Wires leading from each end of the conductive layer were attached to the acquisition 
module as shown in Figure 106. The protective layer was not applied on top of the silver paint as the coupon was not to be exposed to harsh environments.

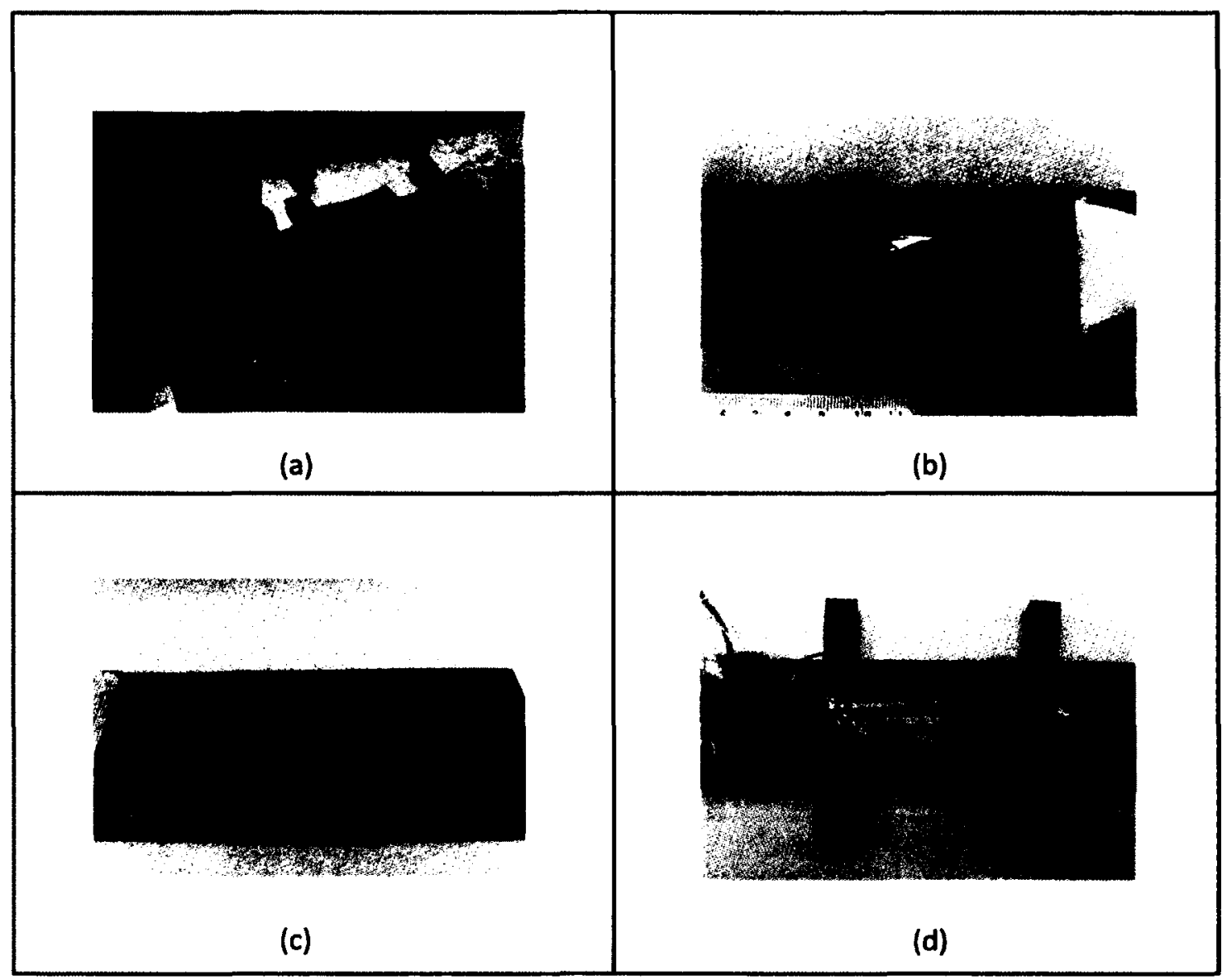

Figure 105: SMCDS: (a) insulating layer installation, (b) Insulating layer, (c) conductive layer installation and (d) completed SMCS 


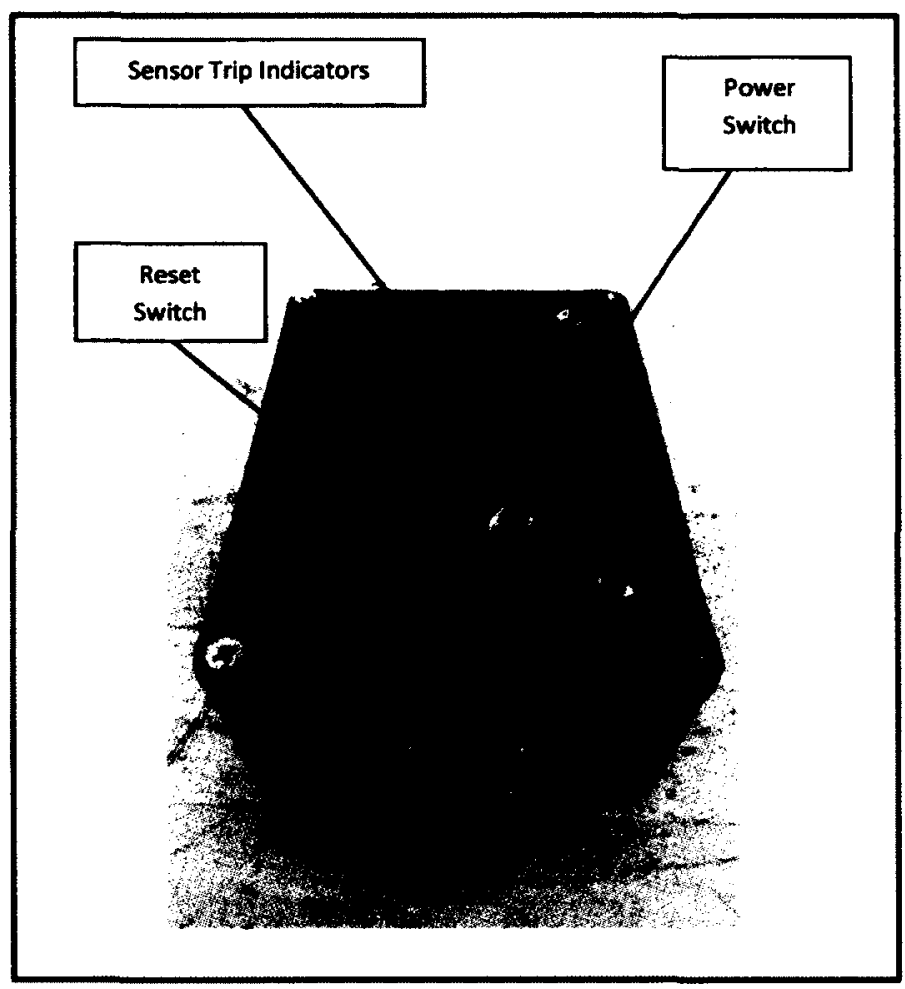

Figure 106: SMCD Acquisition Module

\subsection{Conclusion}

To evaluate the capabilities of multiple SHM suites for the detection of crack and disbond growth, three coupon sets were designed. The first set of coupons was made of aluminum and had a center surface crack; these coupons were for the evaluation of SHM systems for their effectiveness in crack growth detection. The second set of coupons was made of an aluminum substrate and a carbon fibre patch. Both the patch and the substrate were bonded together; these coupons were for the evaluation of SHM systems for their effectiveness in crack and disbond growth detection. The third set of coupons was made of a carbon fibre substrate and a boron fibre patch. Both the patch and the substrate were bonded together; these coupons were for the evaluation of SHM systems for their effectiveness in disbond growth detection. Coupon set 1 and set 2 were designed and manufactured prior to the start of this research while coupon set 3 was designed and manufactured through the course of this research. 
Coupon set 3 was designed to have a local area of high stress that would cause disbond within that given region. This coupon design was done through the aid of ABAQUS ${ }^{\bullet}$ FEA software. Three models were constructed within ABAQUS : a conventional shell model, a continuum shell model and a 3D stress model. The conventional shell model utilizes conventional shell elements which are used to compute planar stresses. Through the use of these elements, computational time was reduced as none of the out-of-plane stresses were calculated. This model was utilized to quickly vary parameters within ply orientation, ply number and geometry in order to determine the optimal coupon design solution. The continuum model was very similar to that of the conventional shell model. Continuum elements do not allow for the calculations of out-of plane stresses; however, unlike the conventional shell element, continuum elements may be stacked through the thickness of the composite component. Results of this model were compared with those obtained using conventional shell model. This model revealed that having a single element through the thickness of each component was adequate for the computation of stresses within the coupon. The 3D stress model was used to apply thermal loads which would have been introduced during manufacturing and to determine if the applied mechanical load would have overcome these thermal residual stresses. This model revealed that stresses within the adhesive layer exceeded the strength of the adhesive and load application would cause a disbond.

While manufacturing both the substrate and the patch, two issues were observed. The first was the formation of bi-phase carbon fibre laminates and the second was fibre displacement within the patches. Bi-phase laminates were formed as a result of slight misalignment of each ply within the layup; this could have been avoided through experience; however, it does not pose a problem for the evaluation of SHM technology. Fibre displacement did pose a problem for experimentation as the patch stiffness was greatly influenced; for that reason trials were 
conducted to solve this issue. Trials revealed that applying a peel ply layer to either side of the patch restricted the motion of fibres during resin flow.

Bonding of the patches to substrates was successful; however, ultrasound C-scans revealed that 4 of the manufactured coupons did not have adequate bonding. These 4 coupons were used for testing as the experimentation was designed to detect disbond and not the strength of the bond.

Acousto-Ultrasonic, CDDT and SMCDS where all instrumented on the coupons. Coupons where split in two groups: SMCS were installed on one group and the CDDT sensors on the other group. However, all coupons were instrumented with Acousto-Ultrasonic sensors. 


\section{Chapter 4.0: Testing and Results}

There were three coupon variants that were tested as part of this research. The first variant (Set 1) was of metallic construction and was designed for the evaluation of an Acousto-Ultrasonic (AU) system for the detection of crack nucleation and growth. The second variant (Set 2), was of metallic and composite construction. This second set was tested under two conditions for the evaluation of the AU SHM system. The first condition was carried out to monitor the nucleation and growth of adhesive failure between the composite and the metallic joint while the second condition was to monitor adhesive failure as well as crack growth within the metallic substrate. The third variant (Set 3) was of all-composite construction, in which a boron fibre patch was adhesively bonded to a carbon fibre substrate. This final set was designed for the evaluation of AU, Capacitive Disbond Detection Technique (CDDT) and Surface Mountable Crack Detection System (SMCDS) for the detection of disbond between two composites.

As the AU SHM technology was the primary focus of this research, $A U$ sensors were instrumented on all coupon variants. CDDT and SMCDS were not evaluated on coupon Sets 1 and 2 , as the designs were unsymmetrical (different patch designs on either side of the coupon) and only allowed the evaluation of a single system. Coupon Set 3 however was of symmetric design and therefore permitted the evaluation of two systems simultaneously. AU sensors were instrumented on a single side while either CDDT or SMCDS were instrumented on the opposing side.

For disbond of patches on coupon Set 2, coupons were subject to static loads for disbond to occur. However, coupons were found to fail near the grips of the test frame and therefore, a fatigue based approach was taken to cause disbond of the patch. There existed no off-the-shelf software that could estimate the life of this coupon based on applied load and therefore loads 
that were used for coupon Set 1 were applied to coupon Set 2. Such loads were found to not cause patch disbond and therefore the fatigue loads where increased to a maximum load of $13,000 \mathrm{lb}$ and a minimum of $1,300 \mathrm{lb}$ which corresponding to a fatigue spectrum of $\mathrm{R}=0.1$. In the case were cracks existed, cracks also grew as disbond occurred. Coupons were allowed to fracture as shown in Figure 107(b).

For disbond of patches on coupon Set 3, an experimental approach was undertaken. Sample coupons were tested to determine the ultimate strength of the coupons as well as ultimate strength of the adhesive used for mounting of all sensors. Coupons were found to fail at $3,800 \mathrm{lb}$ while sensor disbond occurred at $1,000 \mathrm{lb}$. However, patch disbond was observed at $2,000 \mathrm{lb}$. Similar to coupon Set 2, a fatigue base approach was adopted so that patch disbond is allowed to propagate while avoiding sensor disbond.

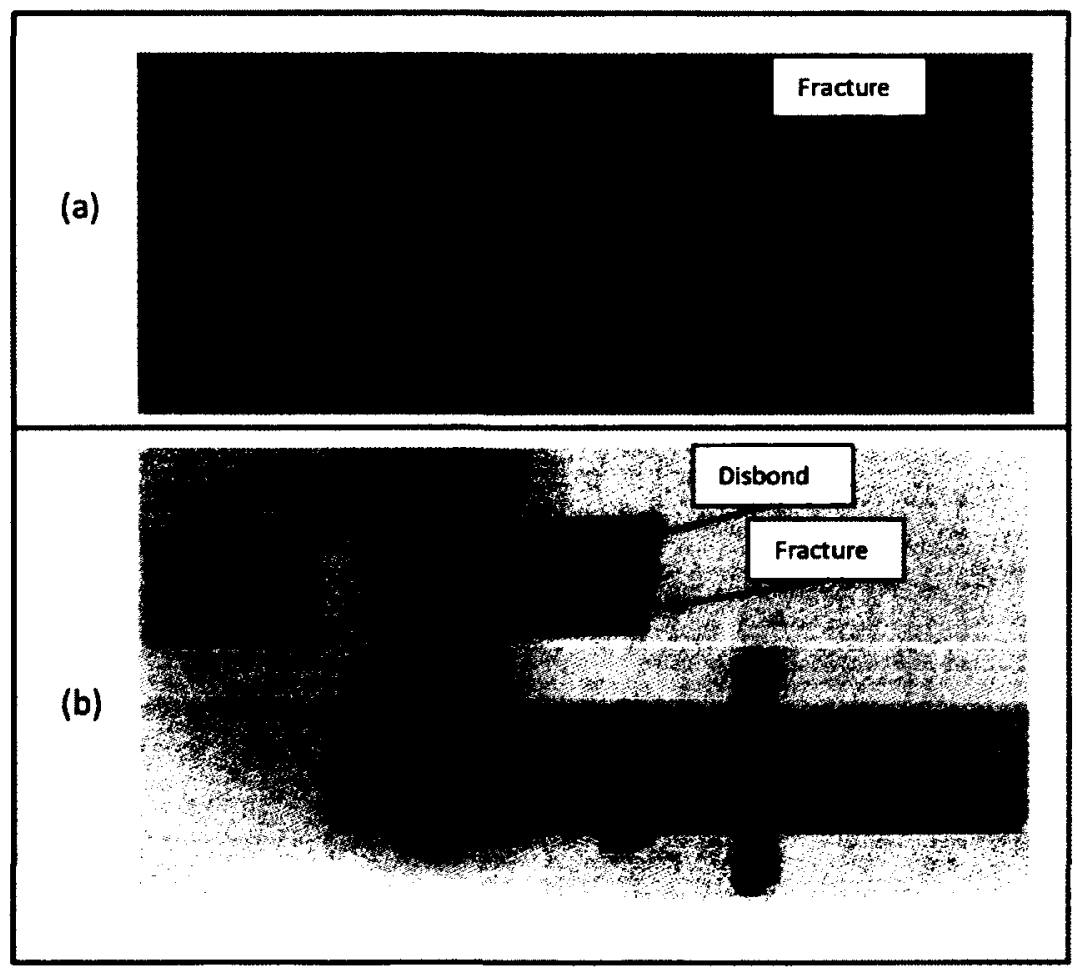

Figure 107: Coupon Sailures: (a) Coupon Set 1 Fracture and (b) coupon Set 2 disbond and fracture 
In total there were 19 coupons tested, 5 for Set 1,4 for Set 2 and 10 for Set 3 . The exact testing procedure, data acquisition and analysis are described within the following subsections. However, not all tested coupons have been described, only the best and worst results are presented within this thesis.

\subsection{Metallic Coupon (Set 1)}

For crack growth on coupon Set 1, initial crack size measurements were obtained using ultrasound C-scans as well as a traveling microscope (a visual inspection technique). The larger of the two crack measurements was inputted into the Air Force Growth (AFGROW) software. AFGROW software uses a conservative approach to calculate fatigue life data based on stress intensity factors, stresses and loading conditions. AFGROW software was used to produce crack length versus life cycle data for multiple analysis using different applied stress conditions. The use of AFGROW allowed for the determination of loads required to induce fracture of the coupon within approximately 100,000 cycles. As a result of this analysis, coupons were fatigued at a maximum load of $10,000 \mathrm{lb}$ and a minimum load of $100 \mathrm{lb}$. Setup for testing under these conditions is described in the following section.

\subsubsection{Apparatus Setup}

Coupons of Set 1 were tested under cyclic conditions with loads between $10,000 \mathrm{lb}$ and $1000 \mathrm{lb}$ which correlate to 20,000 PSI and 2000 PSI, respectively. Inspection of the coupon's health for this set was executed through the use of an AU SHM and a traveling microscope NDE systems.

Coupons were placed within a test frame and were mounted at two attachment points, one at the top and the other at the bottom of the coupon. Grips used for the application of force were 
attached to the coupon at the two attachment points as shown in Figure 108. A traveling microscope was placed such that the coupon and crack were directly within the view of the viewfinder as shown in Figure 108. AU sensors were wired to the ScanGenie ${ }^{\bullet}$ system for data acquisition and setup as described in section 4.1.2.

Coupons were cycled at a frequency of $10 \mathrm{~Hz}$ and after every 5,000 cycles, the test frame was paused and crack measurements as well as data acquisition scans using the $\mathrm{AU}$ systems were gathered. This process was repeated until the number of cycles reached 40,000 . After 40,000 cycles, data collection was carried out every 2,500 cycles; this was done as the crack growth rate increased as the crack grew.

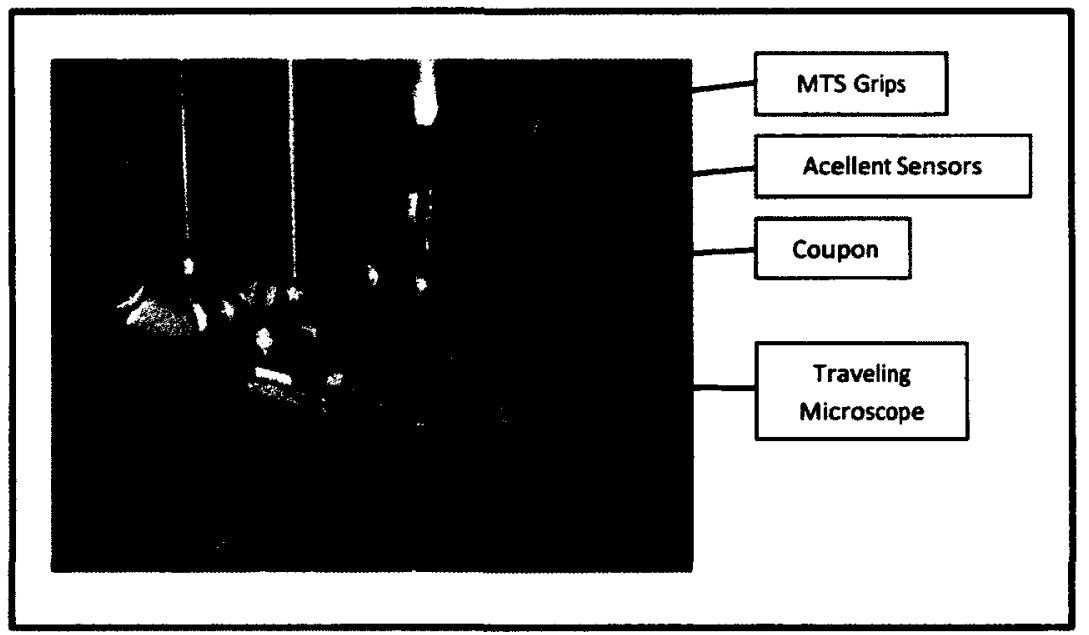

Figure 108: Experimental Setup for Coupon Set 1

\subsubsection{Software Setup}

Monitoring coupons using $A U$ technology was done through the use of the Acellent Technologies ScanGenie ${ }^{\bullet}$ system and its associated SmartPatch ${ }^{\bullet}$ application. Functionality and setup of the SmartPatch ${ }^{\bullet}$ application have been described in section 2.2.3.3.1. However, there exist minor setup procedures that differ from coupon to coupon which include sensor layout as well as path definitions. 
For coupons of Set 1, there existed two experimental sensor configurations that were utilized. Different sensor configurations correlated to different scanning paths and therefore scanning areas. Two distinctly unique configurations were used to determine if greater accuracy can be achieved for damage detection. For each sensor configuration, sensor locations were defined with respect to each other using a Cartesian coordinate system within the SmartPatch application as previously explained within section 2.2 .3 .3 .1 . Through sensor location setup, a coupon as shown in Figure 109(a) was reconstructed to form a digital mapping within the application as shown in Figure 109(b). Such representations were used by the application to interpret and highlight damage location.

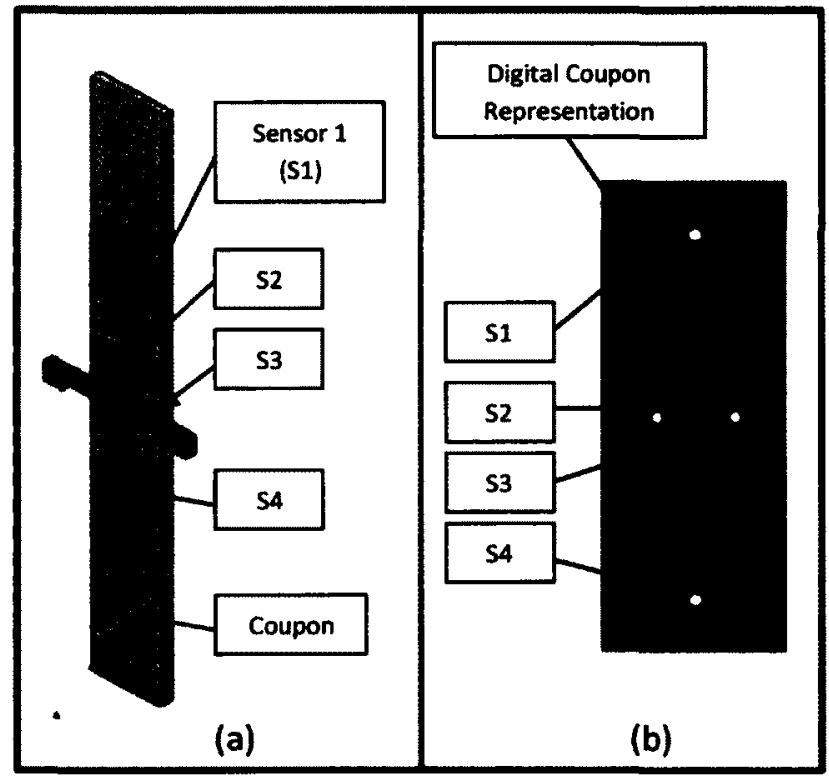

Figure 109: Coupon: (a) Actual coupon and (b) SmartPatch ${ }^{\bullet}$ coupon digital mapping representation

Multiple signal paths were defined within the application (as per section 2.2.3.3.1) to determine areas which will be monitored for damage. Path definition differed from coupon to coupon as signals were highly sensitive to change in piezoelectric element adhesion thickness, fibre alignment and many other factors which are not discussed. Each path was defined having 5 
signal bursts, a $50 \mathrm{~V}$ excitation voltage and $20 \mathrm{~dB}$ for signal strength. Paths were also scanned at frequencies ranging from $100 \mathrm{KHz}$ to $350 \mathrm{KHz}$ with step intervals of $50 \mathrm{KHz}$. Scanning at multiple frequencies was carried out as the optimal frequency for only 2 mode excitation was unknown.

Analysis of Lamb waves as described section 2.2.3.3.2 was done through the correlation between current signal data and that of benchmark signal data. For the benchmark data, paths which were defined earlier were tuned so that the amplitude of each received signal was between $0.5 \mathrm{~V}$ and $1.0 \mathrm{~V}$. Manual increase or decrease of signal gain was applied to signals to transform them from a state as shown in Figure 110(a) to that shown in Figure 110(b). The change in amplitudes between the two signals shown in Figure 110 corresponded to a difference in signal energy which was to be used during data reduction.

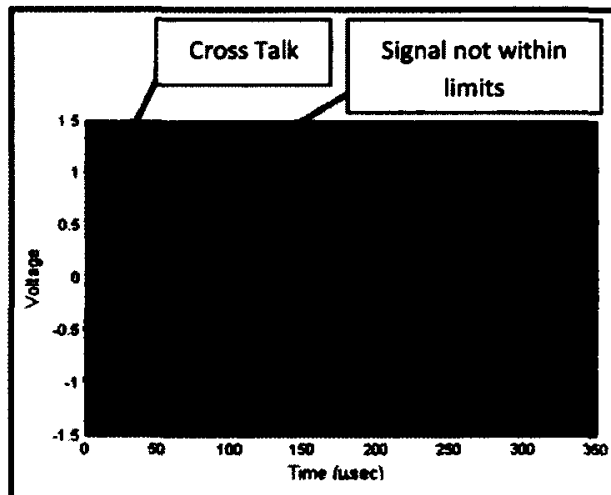

(a)

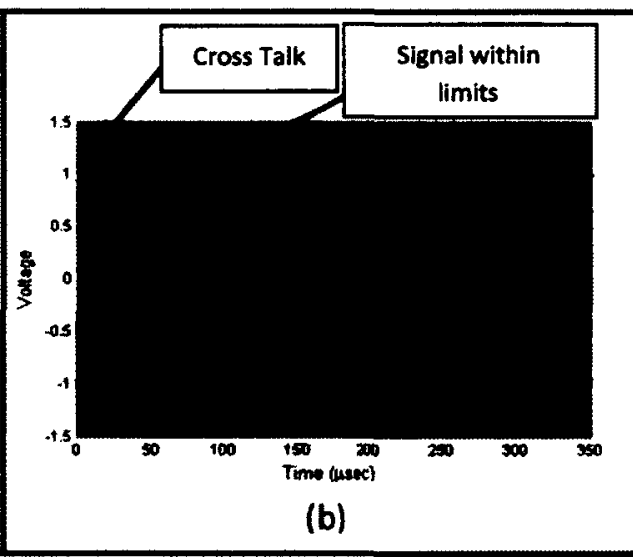

Figure 110: Signal Propagation Settings

\subsubsection{Results}

All coupons of Set 1 were tested utilizing the same procedure. There existed two methods of data collection: AU SHM and visual inspection utilizing a travelling microscope. Throughout the course of this research, measurements as obtained using the traveling microscope were utilized to validate results which were obtained using the AU SHM system. 
The Acellent ScanGenie (AU SHM) system was equipped with the SmartPatch application for the analysis of signal data. The SmartPatch application utilized two separate diagnostic and prognostic utilities: Direct Path Image (DPI) and Semi-Empirical Damage Sizing (SEDS) Utilities. The DPI utilizes the digital representation of the coupon to identify the location of defects while the SEDS utility interprets damage size. Each utility was run independent of the other and therefore the results for each is presented independently in the following sections.

\subsubsection{Direct Path Image (DPI) utility results}

The DPI utility is a powerful tool which was designed to allow for quick assessment of damage initiation. The DPI utility utilizes the digital mapping of the coupon and displays damage in the form of discoloration as shown in Figure 112. Identification of damaged areas was done by comparing current signal data to that of the benchmark signal data.

Coupons which were analysed using the DPI utility were subjected to crack growth from approximately 0.2 inches in length to approximately 1.0 inch prior to full fracture. The DPI utility identified crack severity and location through the use of a color gradient as shown in Figure 111. The color gradient ranges from dark blue to dark red. The blue on the scale is considered undamaged while red represents severe damage.

Results showing progression of damage are presented for two coupons: SPP2-12 and SPP2-15 within this section. Both coupons utilized the same 4 sensor configuration and therefore utilized a very similar digital coupon representation. Multiple DPI utility images were captured throughout the life of each coupon; each corresponding to a different crack length. DPI utility images at 5K, 20K, 40K, 55K, 70K and 80K for SPP2-12 were captured and are presented within Figure 112. Crack detection on the coupon was observed as a change of color at the center of 
the coupon where the crack resides. Progression of the crack was also observed as a change of color from blue to yellow when examining images within Figure 112. Images on the left denote smaller crack size as indicated by the crack measurement located at the top of the images while images on the right denote larger crack measurements.

The second coupon (coupon SPP2-15) utilized the same sensor configuration as that used on the first coupon (SPP2-12). DPI utility images for SPP2-15 were captured at 5K, 20K, 40K, 55K, 75K and $80 \mathrm{~K}$ and are presented in Figure 113 . The crack was located between the two middle sensors as indicated in Figure 113. Crack identification and severity was presented using the same color gradient as that explained for coupon SPP2-12. Changes in crack severity were observed from right to left within Figure 113. Unlike SPP2-12, crack identification for SPP2-15 was not as accurate. The crack which was located between the two centre sensors was identified as being located just below the actual location as shown in Figure 113; making location identification inaccurate.

The third and final coupon tested as part of this set was SPP2-26. SPP2-26 was equipped with a completely different sensor configuration than SPP2-12 and SPP2-15. The sensor configuration used on SPP2-26 utilized 8 sensors which were bonded to the aluminum. The use of 8 sensors as opposed to 4 was done to determine if greater damage detection accuracy could be obtained. During testing of the coupon, sensors disbonded from the aluminum; this occurred at less than 40,000 cycles. Due to sensor disbond, signal propagation properties were affected and therefore current signals could not be evaluated using the benchmark signals. Images for SPP2-26 have not been included below as they did not provide valuable data for the evaluation of the SHM system. 


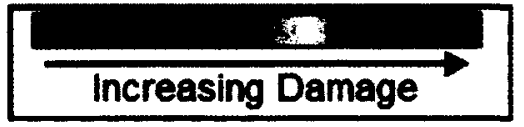

Figure 111: Damage Spectrum

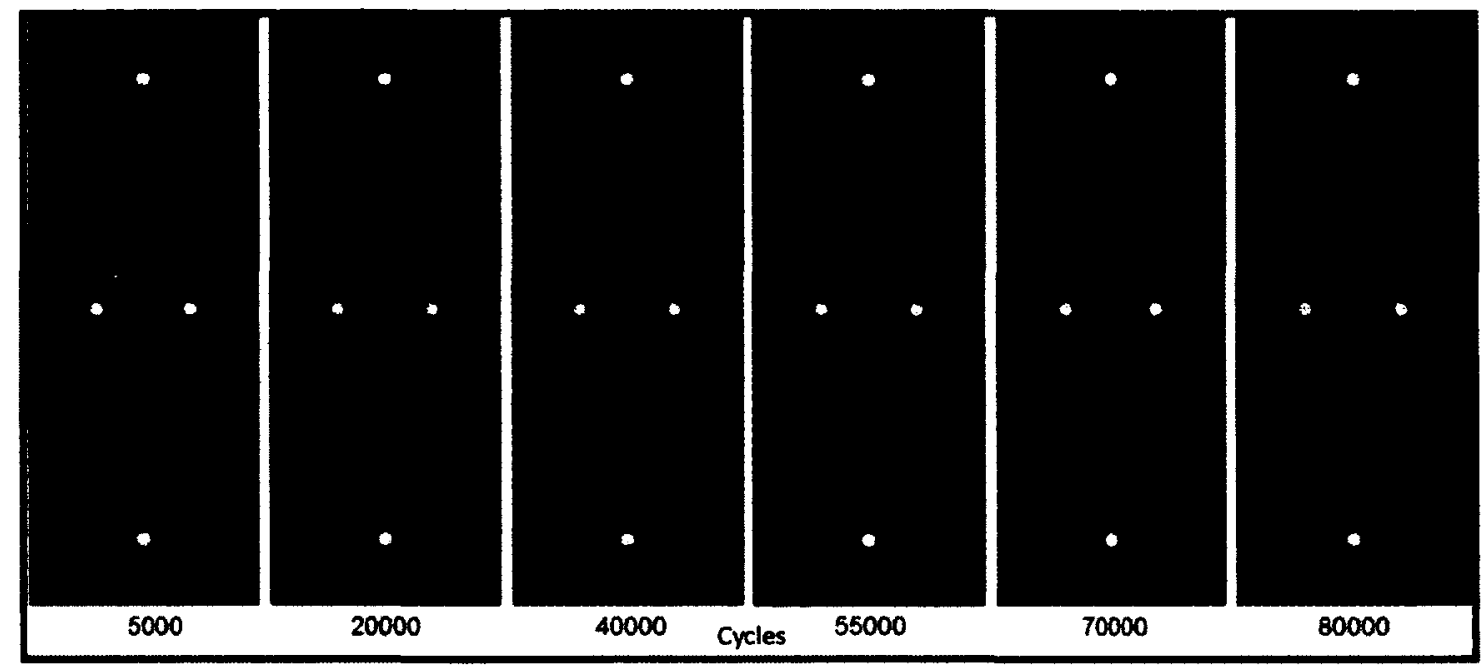

Figure 112: Coupon SPP2-12 Direct Path Image Results at a 0.5 Threshold

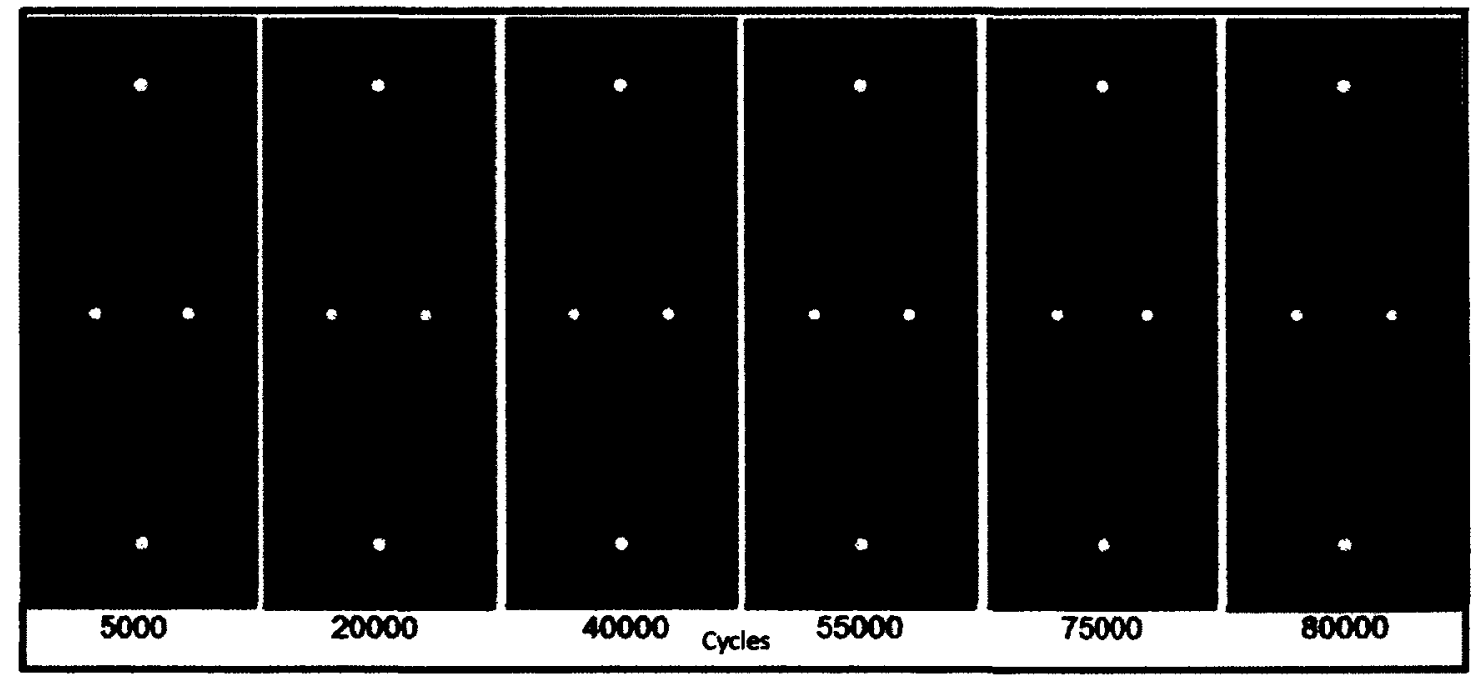

Figure 113: Coupon SPP2-15 Direct Path Image Results at a 0.5 Threshold

Once the presence and location of damage has been determined using the DPI utility, the SEDS was applied to determine the size of such damage; this is presented in the following section. 


\subsubsection{Semi Empirical Damage Sizing (SEDS) results}

The SEDS utility is one of the two utilities found within the SmartPatch application and its purpose was to determine crack lengths at all inspection points throughout the coupon life cycle. The SEDS utility was able to analyze signal data and provided an "interpreted" damage size; in this case, crack length. Interpreted crack lengths were a result of Damage Indices (DI), which were described in more detail within section 2.2.3.2. Damage Indices were obtained by creating a Bspace file and by providing known crack lengths of the first few inspections to the application. The Bspace is a collection of signal files into a single file or file database for which current signals may be compared against for damage. For SPP2-12, SPP2-15 and SPP2-26 approximately $40 \%$ of all collected signal data was assigned to the Bspace file. Multiple analyses were executed for each coupon, each with a different number of known crack length inputs.

For coupon SPP2-12, multiple analyses using the SEDS utility were executed, each with a different known crack length inputs. Analysis for the interpretation of crack lengths was executed with as little as 9 and as high as 21 known crack length inputs (as measured by the traveling microscope). In total, there were 26 signal data files gathered for this coupon and the SEDS utility was analyzing all signal data against the Bspace to determine crack lengths which were not provided to the software. Figure 114 shows crack interpretations for analysis with 9 (40,000 cycle curve), 11 (45,000 cycle curve), 13 (50,000 cycle curve) and 17 (60,000 cycle curve) crack length data point inputs. In addition to the analysis curves, two other curves exist: the actual crack length as measured by the traveling microscope and AFGROW estimates. Recalling, AFGROW software was used earlier to produce crack length versus life cycle data for each coupon. While examining each of the curves produced by the SEDS utility within Figure 114, it is evident that an increase in the number of known crack length inputs resulted in crack length 
interpretations that are closer to that of the actual measured crack lengths. All of the generated SEDS curves were also found to over-interpret crack lengths; however, all such interpretations were less than estimates produced using AFGROW. Within Figure 114, there existed one overinterpreted point found at 55,000 cycles on all SEDS curves and is highlighted as an "interpretation spike". The interpretation spike appeared to be an outlier as compared to all other interpretations and may be a result of the polynomial approximations used within the algorithm for the construction of the curves.

Similar to coupon SPP2-15, multiple analyses were executed using the SEDS utility, each with a different quantity of known crack length inputs. Analyses for the interpretation of crack lengths were executed with as little as 9 and as high as 20 known crack length inputs. In total, there were 29 signal data files gathered for this coupon and the SEDS utility was used to determine crack lengths which were not provided to the software. Figure 115 shows crack interpretations for analysis with 11 (45,000 cycle curve), 15 (55,000 cycle curve), 19 (65,000 cycle curve) and 21 $(70,000$ cycle curve) crack length data point inputs. Similar to the results for SPP2-12, all the SEDS curves were plotted along with the measured crack lengths as well as AFGROW estimates. Examination of each of the curves produced by the SEDS utility within Figure 115 revealed the same trend as that observed for coupon SPP2-12 within Figure 114. This trend indicates that an increase in the number of known crack length inputs results in interpretations that are closer to that of the actual measured crack length. Within Figure 115, there existed multiple interpretation spikes which consistently over-predict the crack length. These multiple interpretation spike may still be as a result of the polynomial approximations. 


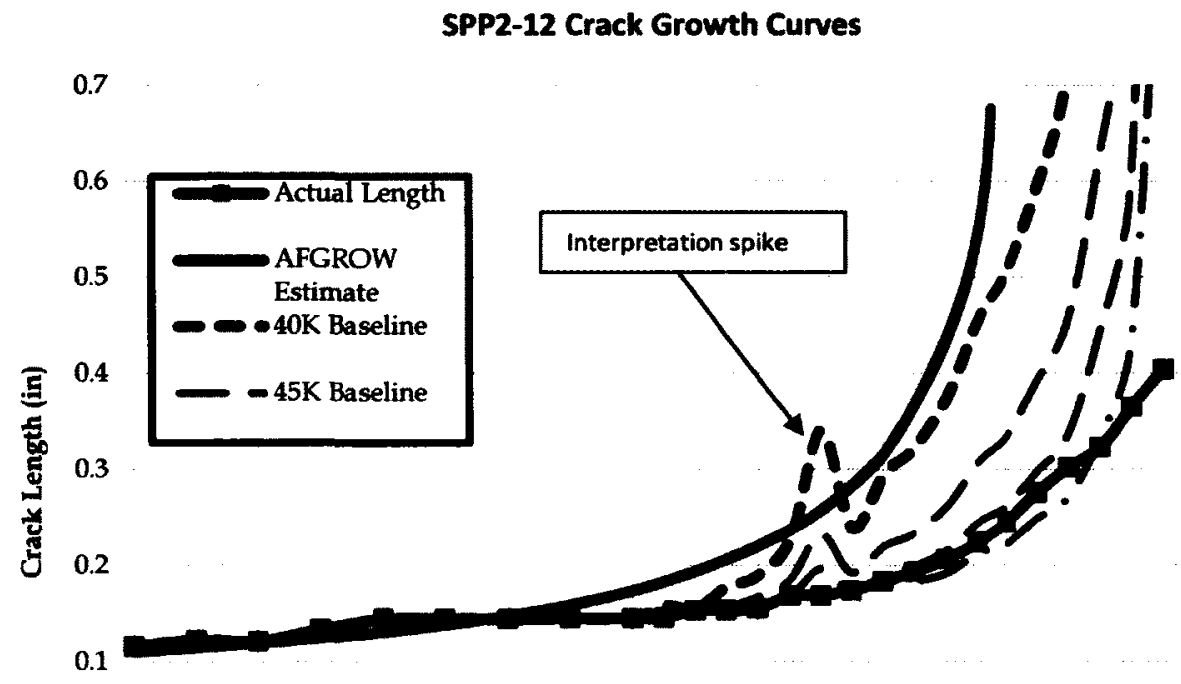
$\circ$

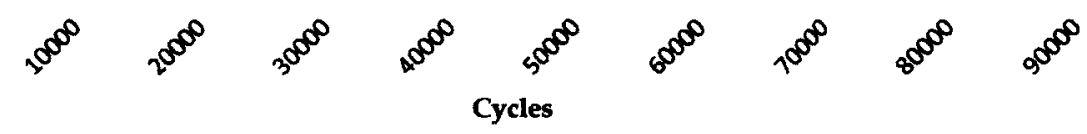

Figure 114: Semi-Empirical Interpretations for Coupon SPP2-12

SPP2-15 Crack Growth Curves

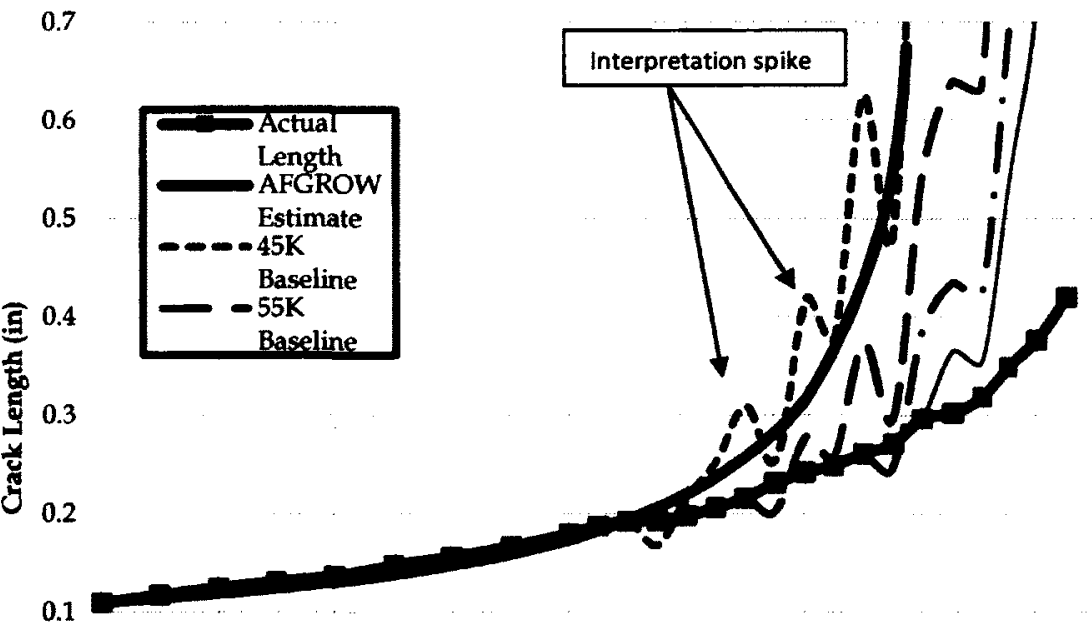

0

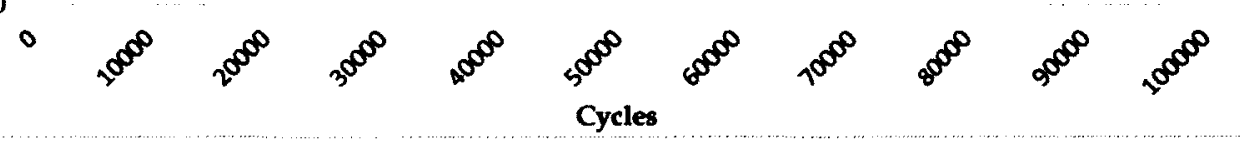

Figure 115: Semi-Empirical Interpretations for Coupon SPP2-15 
The final coupon tested for Set 1 was SPP2-26. As previously mentioned, SPP2-26 had a different sensor configuration as compared to all other tested coupons. During testing of coupon SPP226, sensors were found to disbond and therefore many signal propagation properties were affected with every loading cycle. Figure 116 shows multiple SEDS utility analysis curves along with the actual measured crack lengths and the AFGROW estimates. Examination of SEDS curves found in Figure 116 reveals many fluctuations within a single curve. Fluctuations over-predict and in some cases under-predict crack lengths. There is no consistency or smooth portion to these graphs and based on these curves, it is clear that sensor disbond greatly affects the system's ability to accurately interpret crack lengths.

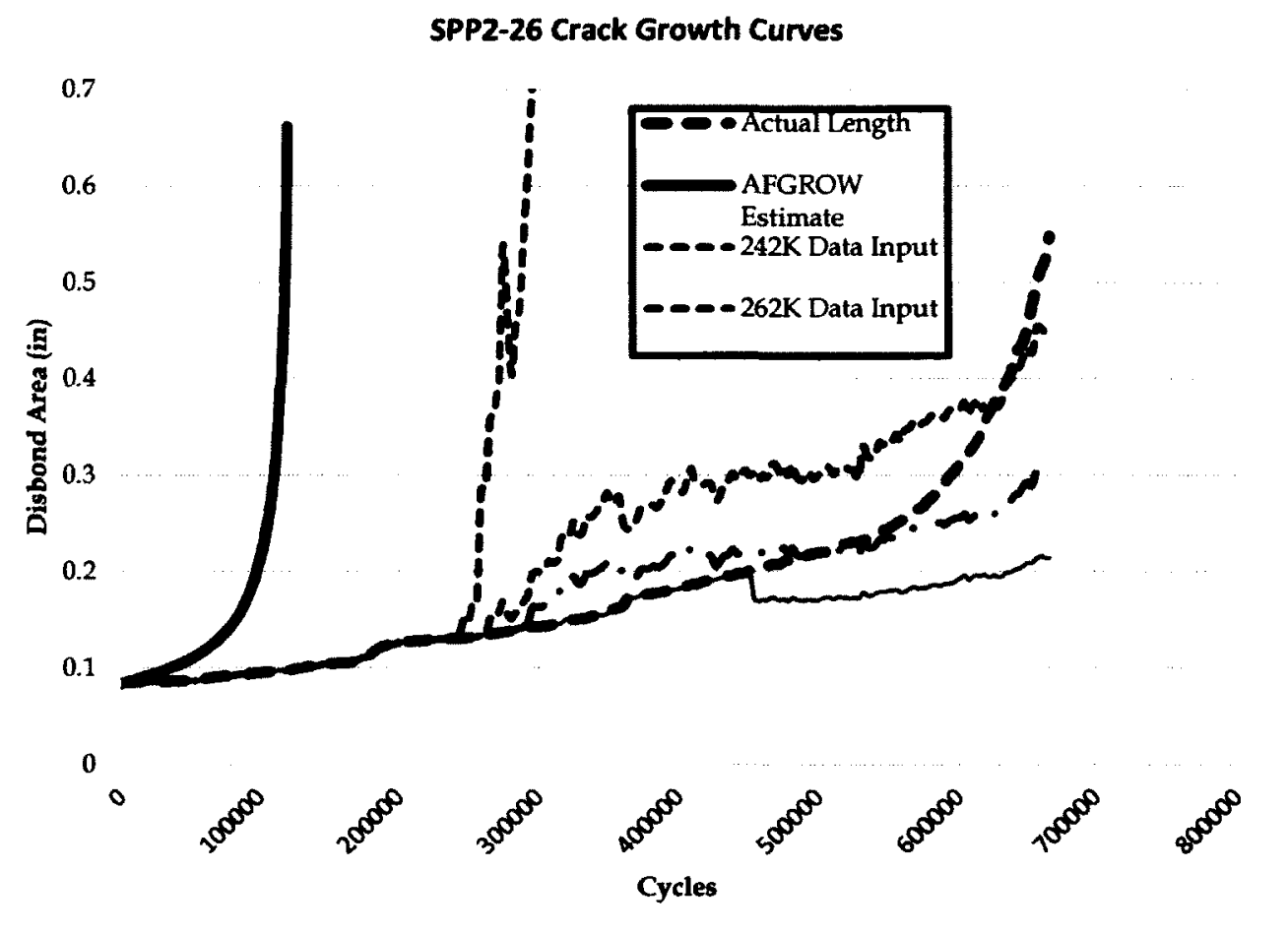

Figure 116: Semi-Empirical Interpretations for Coupon SPP2-26 


\subsection{Composite to Metallic (Coupon Set 2)}

Coupons of Set 2 were designed for the evaluation of the AU SHM system for the detection of disbond initiation and growth. This coupon set was intended to disbond under static loading; however, during testing it was found that the coupons failed near the test frame grips. To induce disbond, a fatigue based approach was utilized. Unlike coupon Set 1, there existed no off-the-shelf software that was similar to AFGROW for estimation of disbond growth. These coupons were initially loaded under the same conditions as those used for coupon Set 1 . After testing, disbond was not induced and the loads were changed to a maximum of $13,000 \mathrm{lb}$ and a minimum of $1,300 \mathrm{lb}$. Under these conditions the failure criteria was set such that $50 \%$ of the patch was allowed to disbond. Setup for the testing under these parameters and conditions is described in the following section.

\subsubsection{Apparatus Setup}

Coupons of Set 2 were tested under cyclic conditions with loads between $13,000 \mathrm{lb}$ and $1300 \mathrm{lb}$ which correlate to 26,000 PSI and 2600 PSI, respectively. Inspection of coupon health for this set was executed through the use of the AU SHM and the pulse thermography NDE systems.

Coupons were placed within the MTS test frame and were mounted at two attachment points similar to that used for coupon Set 1 as shown in Figure 117. An infrared camera and two xenon flashes (thermography system) were positioned such that the infrared camera was focused on the patch as shown in Figure 117.

Coupons were cycled at a frequency of $10 \mathrm{~Hz}$ and after every 2,500 cycles, the test frame was paused and thermography images as well as data acquisition scans using the AU system were gathered. This process was repeated until the number of cycles reached 20,000 . After 20,000 
cycles data collection was carried out every 5,000 cycles; this was done as disbond rates decreased as the patch disbond area increased.

Software setup for the Acellent system was done in a similar manner as that used for coupon Set 1 and as described in section 4.1.2.

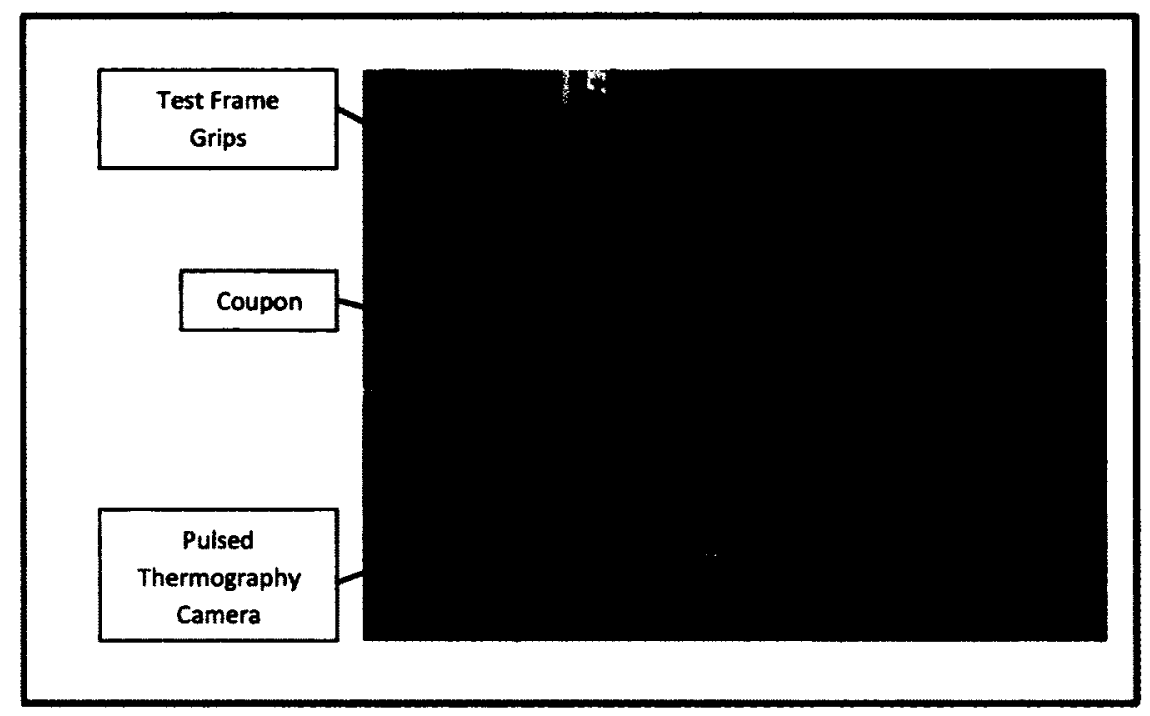

Figure 117: Experimental Setup for Coupon Set 2

\subsubsection{Results}

A total of four coupons were tested as part of Set 2. All coupons were of carbon patch bonded to an aluminum substrate design. Of all the manufactured coupons, three of the four coupons had center surface cracks underneath the patch. All coupons of Set 2 were tested utilizing the same procedure as described in section 4.2.1. There existed two methods of data collection during testing: AU SHM and pulse thermograph NDE. Throughout the course of this research, disbond area measurements were obtained using the thermography system and were used to validate the results of the AU SHM system. For coupons with center surface cracks, crack lengths were not validated as there were no means of obtaining the measurements without removal of the coupon from the test frame. 
Thermography images, like those shown in Figure 118 are constructed by comparing gathered images with benchmark images which were captured prior to the initiation of disbond. A difference of surface temperatures can be illustrated as shown in the figure. The red color within the image indicates little change between temperatures of the benchmark and the current images while blue indicates a change of temperatures between benchmark and the current images. Therefore, blue denotes disbonded area while red denotes bonded area. Calculation of the disbonded area was done by outlining the blue area as shown in Figure 118. Through the use of an area calculation algorithm, disbond area measurements were estimated. Thermography images as well as their estimated disbond areas were used to validate the DPI and the SEDS utility respectively presented in the following sections.

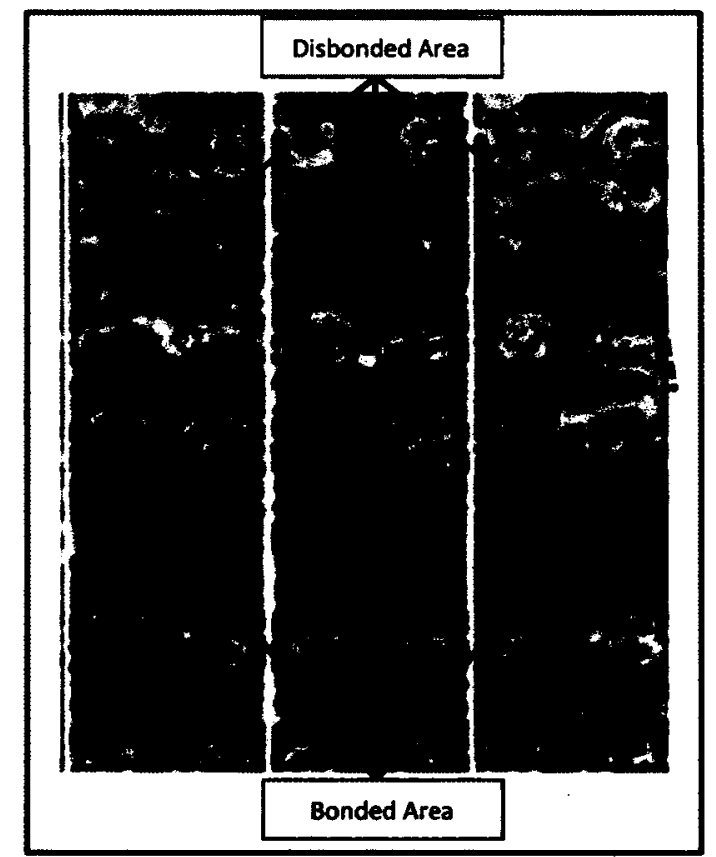

Figure 118: Estimated Disbond Areas using Thermography

\subsubsection{Direct Path Image (DPI) utility results}

There were four coupons analyzed using the DPI utility: SPP2-11, SPP2-13, SPP2-14 and SPP2-18. Coupons SPP2-14 and SPP2-18 were equipped with the same sensor configuration while SPP2- 
11 and SPP2-13 were equipped with their own unique sensor configurations. Therefore, each DPI utility image looks different and is analysed differently.

Coupon SPP2-11 was constructed of a carbon patch and aluminum substrate with no center surface crack within the aluminum. The coupon was instrumented with $7 \mathrm{AU}$ sensors and wired to the ScanGenie ${ }^{\bullet}$ system. The placement of 7 sensors allowed for many scanning paths; analysis using the DPI utility was carried out using all paths and in some cases only a few paths. Through analysis of specific paths and their associated signal data, smaller portions of the entire coupon were analyzed and are denoted as Zones as shown in Figure 119. Figure 119 shows coupon SPP2-11 with three analysis zones. The three zones shown in the figure allowed for examination of only the patch tip (Zone 1), the base of the patch (Zone 2) or the summation of the two (Zone 3).

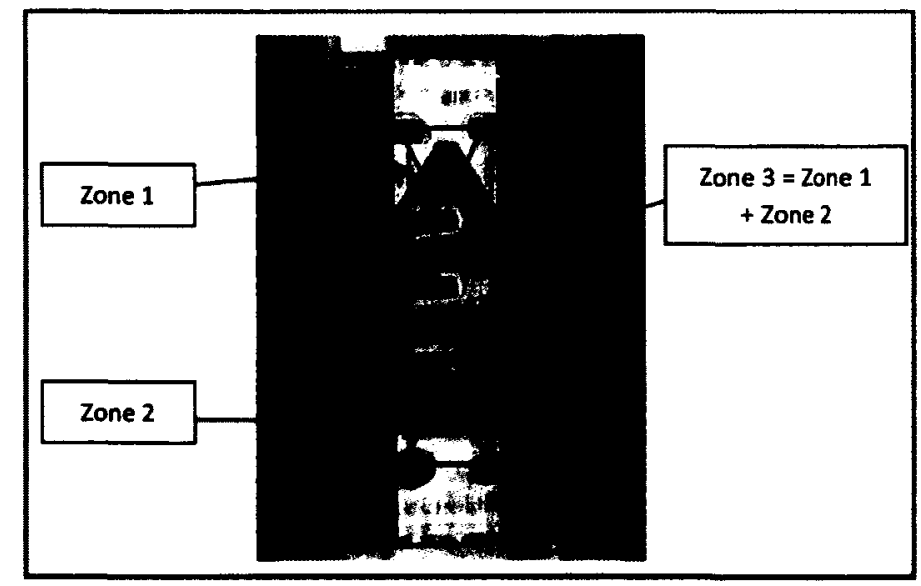

Figure 119: SPP2-11 Scanning Zones: (a) Zone 1, (b) Zone 2 and (c) Zone 3

Zones 3, Zone 1 and Zone 2 were analyzed and results are presented in Figure 120(a), Figure 120(b) and Figure 120(c), respectively. In addition to the DPI utility images, thermography images indicating disbond are also found in Figure 120. Figure 120(d) shows disbond progression through the lifecycle of SPP2-11. Comparing the thermography images to DPI utility images from Figure 120 reveals that disbond progression as observed using thermography images does not 
correlate with that indicated using Zone 3 analysis. Rather, Zone 3 analysis indicates large disbond in as early as 40,000 cycles and is found beneath the entire surface of the patch. However, Zone 1 analysis reveals that damage progression as indicated through the DPI utility correlates to that of the thermography images and disbond location is indicated accurately. Analysis of Zone 2 using DPI utility indicates disbond at the bottom side of the patch, which is accurate. However, comparing Zone 1 and Zone 2 analysis to each other reveals that according to the highlighted areas, disbond areas found in Zone 1 and Zone 2 should be identical in size; this is however not true as thermography images show major disbond at the tip and little disbond at the base. 


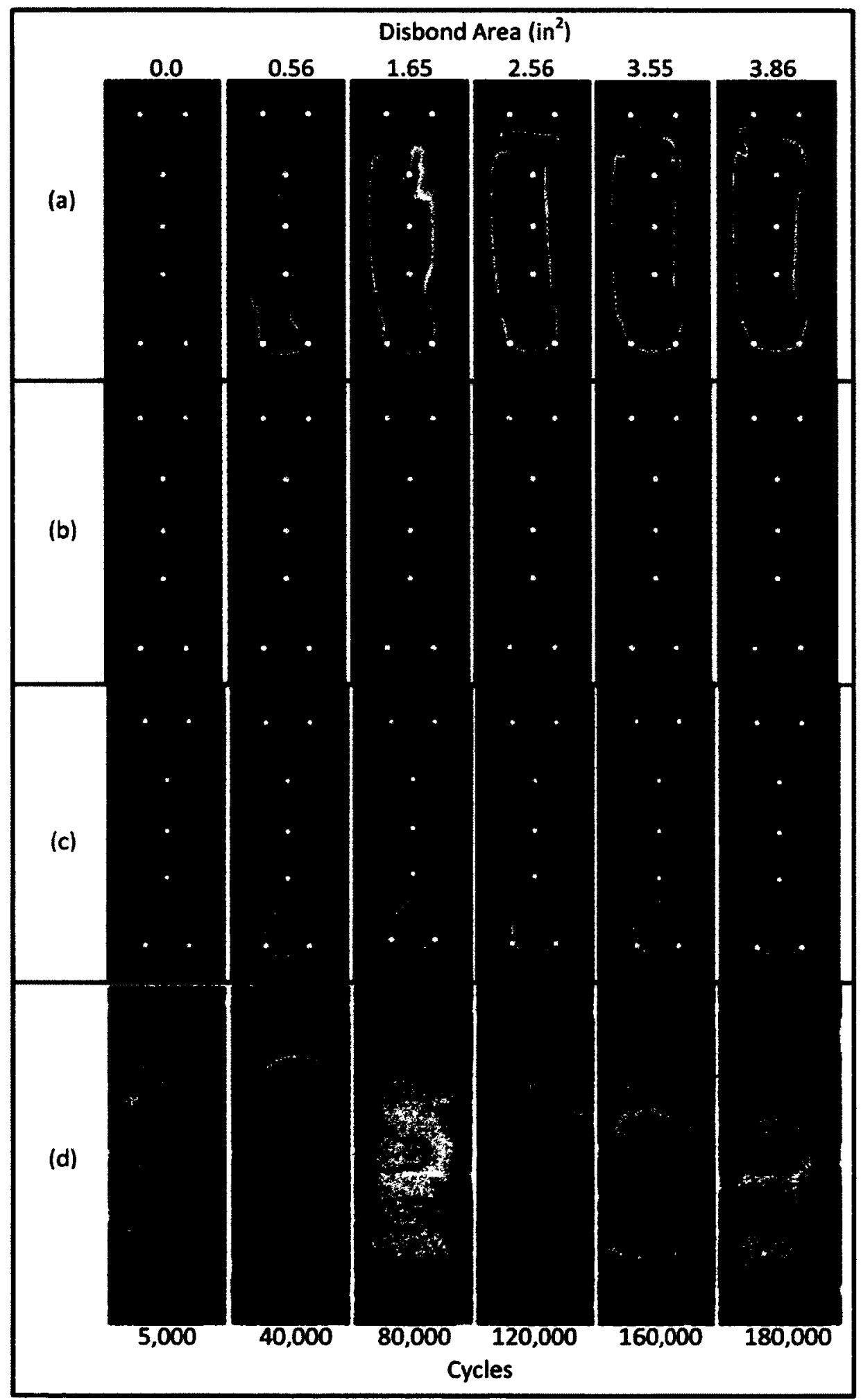

Figure 120: Coupon SPP2-11: (a) DPI for all zones at a 0.5 threshold, (b) DPI for zone 1 at a 0.5 threshold, (c) DPI for zone 2 at a 0.5 threshold and (d) Thermography images 
Coupon SPP2- 13 was the second coupon tested as part of this set; this coupon contained a center surface crack underneath the bonded patch. For this coupon, two forms of damage progression existed: patch disbond and crack growth. Coupon SPP2-13 had a different sensor configuration than that of SPP2-14 and SPP2-18 which were of identical coupon design. The sensor configuration used on SPP2-13 utilized 10 sensors which allowed for multi-zone analysis. Four zones were analyzed using the DPI utility; Zone 1 , Zone 2, Zone 3 and Zone 4 each of which focuses on a specific section of the coupon. Zone 1, Zone 2, Zone 3 and Zone 4 focus on the tip of the patch, the center of the patch, the base of the patch and the entire coupon respectively as shown in Figure 121. DPI utility images for all zones along with thermography images are presented in Figure 122. All DPI utility images as well as thermography images from Figure 122 were captured at $5,000,20,000,40,000,55,000,70,000$, and 80,000 cycles.

Each zone's DPI utility images were analysed independently and obtained results were compared to thermography images for validation. Examination of Zone 4 DPI utility images for all cycles indicates disbond of the entire patch in as early as 55,000 cycles; however, the largest captured disbond area as validated by thermography and shown in Figure 122 occurs at 80,000 cycles. Examination of Zone 1 and comparing its results to the thermography images reveals that there is a correlation between the actual disbond area and the disbond area as indicated by the DPI utility. Increase in disbond area is accurately indicated in the location of the patch tip with varying intensity as shown in Figure 122(b). Unlike results for Zone 1, results for Zone 2 are influenced by both crack growth as well as disbond. Zone 2 images are shown in Figure 122(c) and indicate significant damage formation while thermography images show no disbond in that region prior to 70,000 cycles. The damage formation as indicated within Zone 2 could be attributed to crack growth alone; however, results are inconclusive as they were not validated using an NDE system. Zone 3, was the final zone analysed for this coupon. Zone 3 focused on 
the base of the patch. It was found that the DPI utility images indicated disbond at the base of the patch; this however is completely inaccurate as there is no evidence of disbond found within the thermography images shown in Figure 122(d).

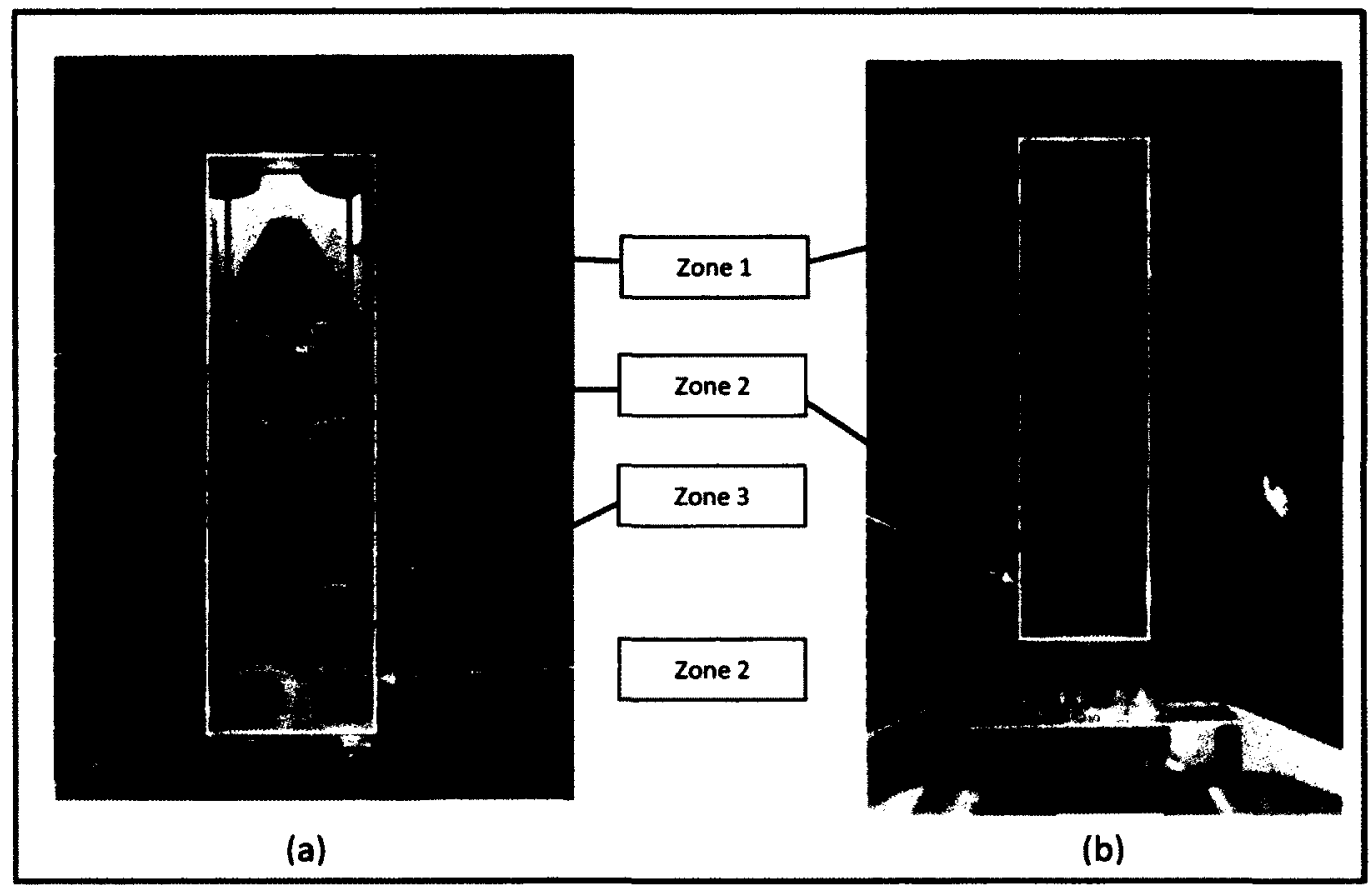

Figure 121: Coupon Scanning Zones: (a) SPP2-13 zones, (b) SPP2-14 and SPP2-18 scanning zones 


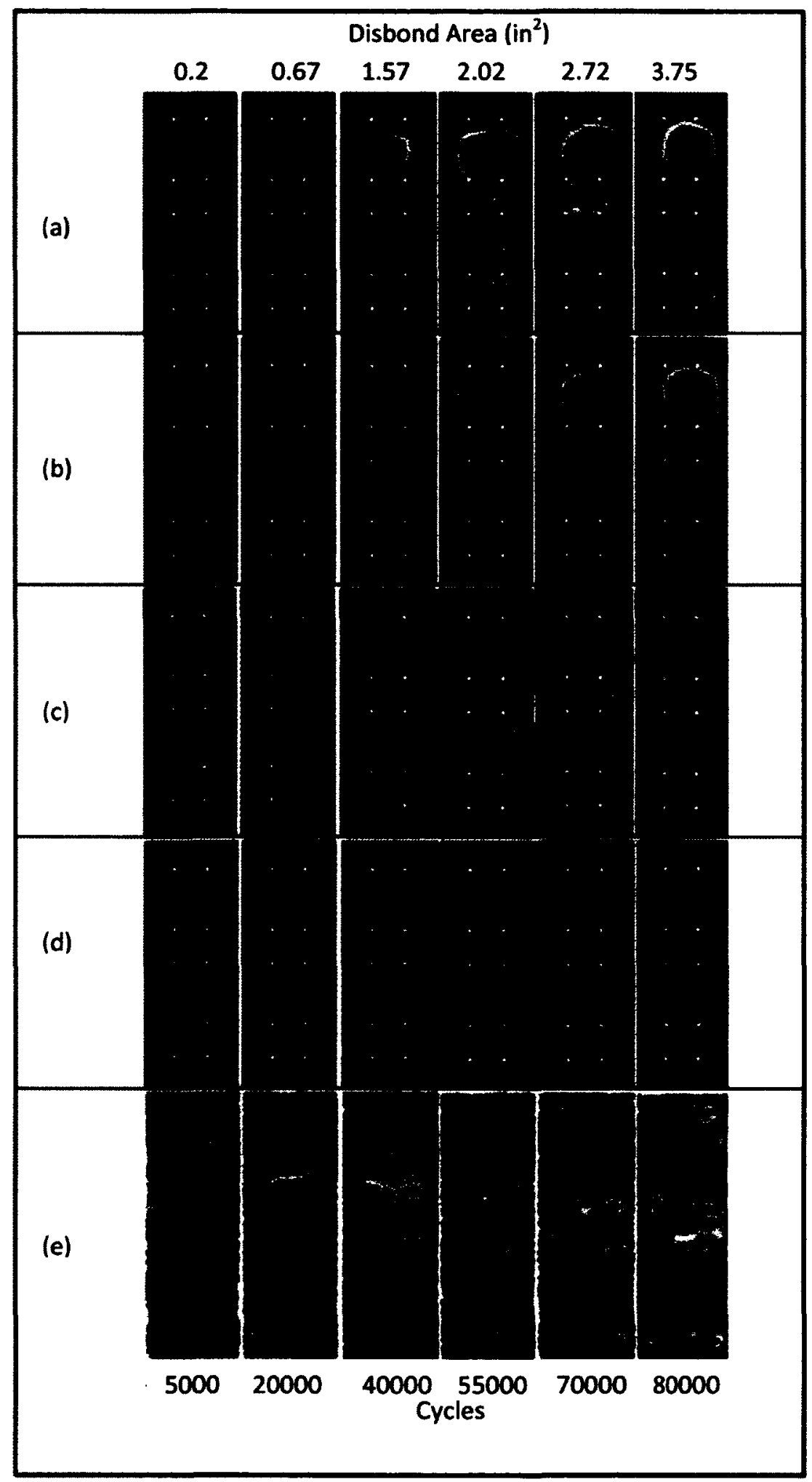

Figure 122: Coupon SPP2-13: (a) DPI for all zones at a 0.5 threshold, (b) DPI for zone 1 at a 0.5 threshold, (c) DPI for zone $2 \& 3$ at a 0.5 threshold, (d) DPI for zone 4 at a 0.5 threshold and (e) Thermography images 
Coupon SPP2-14 and SPP2-18 were the final two coupons tested within this set; both were equipped with 6 sensors and an identical sensor configuration. The use of a 6 sensor configuration allowed for multi-zone analysis. Similar to coupon SPP2-13, both of these coupons experienced both crack growth and patch disbond. The 6 sensors were used to monitor three zones. Zone 1, Zone 2 and Zone 3 focus on the tip of the patch, the base of the patch and the entire coupon, respectively, and are all shown in Figure 121. The DPI utility images for all zone analyses as well as thermography images for coupon SPP2-14 are shown in Figure 123; the DPI utility images for SPP2-18 are not presented within this chapter as they are similar to those of SPP2-14.

Each zone's DPI utility images were analysed independently and results of which were compared to thermography images for validation. Examination of Zone 3 DPI utility images for all cycles indicates disbond of the entire patch in as early as 40,000 cycles. However, the largest captured disbond area as validated by thermography and as shown in Figure 123 (d) occurs at 80,000 cycles and is approximately $20 \%$ of the overall patch area. Examination of Zone 1, while comparing its results to the thermography images, reveals that there is a correlation between the actual disbond area and the disbond area as indicated by the DPI utility. Increase in disbond area is indicated in the location of the tip of the patch with varying intensity as shown in Figure 123(a). Zone 2, was the final zone analysed for this coupon. Zone 2 focused on the base of the patch. It was found that the DPI utility images indicated disbond at the base of the patch; this however is completely inaccurate as there is no evidence of disbond found within the thermography images shown in Figure 123(d). 
After examining coupons SPP2-11, SPP2-13, SPP2-14 and SPP2-18 using the DPI utility for damage identification, the SEDS was applied to determine the size of such damage; this is presented in the following section. 


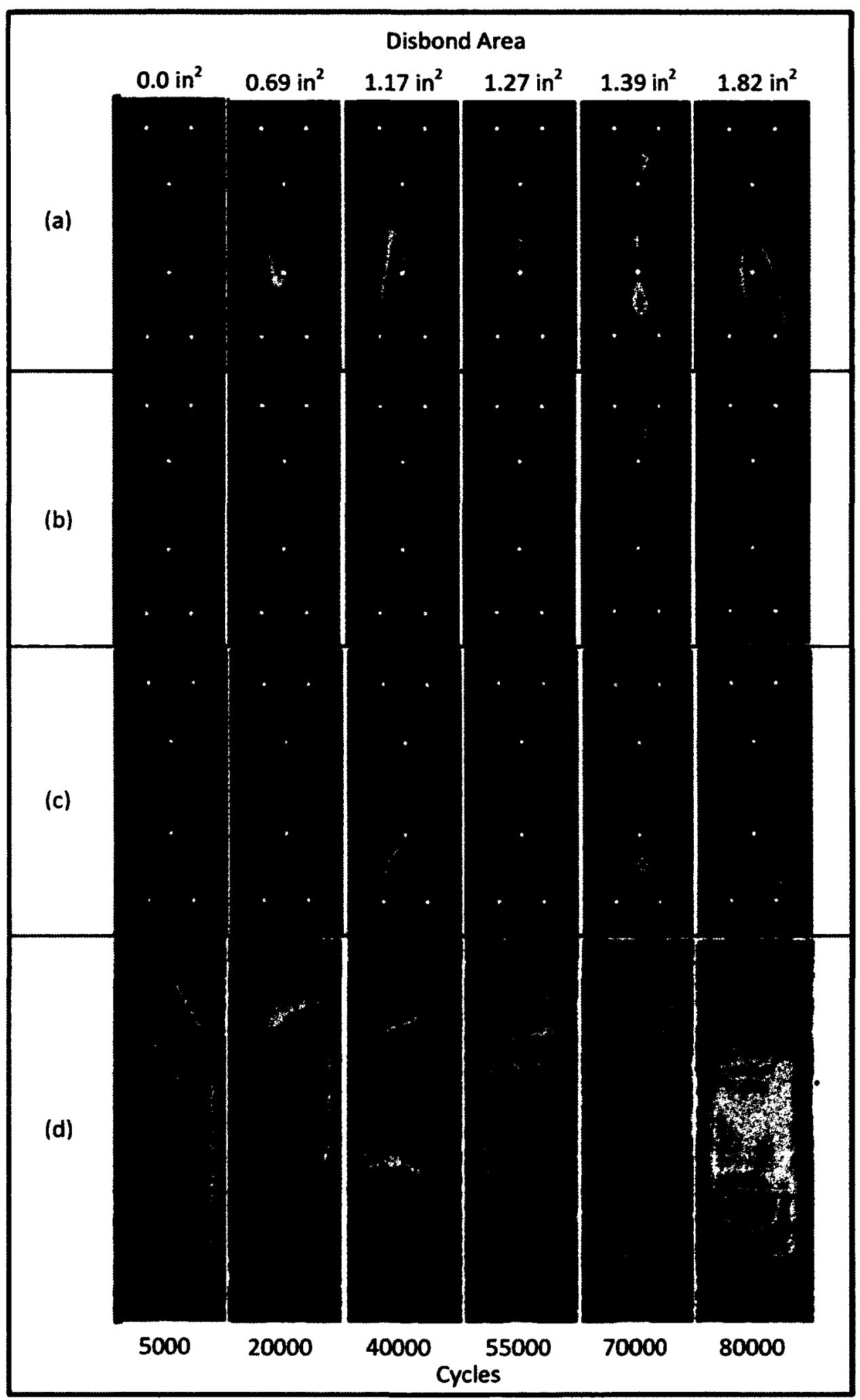

Figure 123: Coupon SPP2-14: (a) DPI for all zones, (b) DPI for zone 1, (c) DPI for zone 2 and (d) Thermography images 


\subsubsection{Semi Empirical Damage Sizing (SEDS) Results}

The SEDS utility is one of the two utilities found within the SmartPatch application and its purpose was determining disbond area at all inspection points throughout the coupon life cycle. The SEDS utility was able to analyze signal data and provides an "interpreted" damage size; in this case, disbond area. Interpreted disbond areas are a result of Damage Indices (DI), which were described in more detail within section 2.2.3.2. Damage Indices were obtained by creating a Bspace file and via providing known disbond areas for the first few inspections to the application. For SPP2-11, SPP2-13, SPP2-14 and SPP2-18 approximately $40 \%$ of all collected signal data was assigned to the Bspace file. Multiple analyses were executed for each coupon, each with a different number of known disbond area inputs.

For coupon SPP2-11 multiple analyses using the SEDS utility were executed, each with different known disbond area inputs. Analyses for the interpretation of disbond area were executed with as little as 13 and as high as $\mathbf{4 1}$ known disbond area inputs (as found using thermography). In total, there were 49 signal data files gathered for this coupon and the SEDS utility was analysing all signal data against the Bspace to determine disbond areas which were not provided to the software. Figure 124 shows disbond area interpretations for analysis with 24 (95,000 cycle curve), 27 ( 110,000 cycle curve), 32 (135,000 cycle curve) and 41 (185,000 cycle curve) disbond area data point inputs. In addition to the SEDS curves, the actual disbond area curve is also plotted within Figure 124. While examining each of the curves produced by the SEDS utility within Figure 124, it is evident that an increase in the number of known disbond area inputs results in interpretations that are closer to that of the actual measured value. Unlike the results for coupons of Set 1, results of SEDS interpretations are inaccurate by a minimum of $45 \%$ and a 
maximum of $375 \%$ when compared to the actual disbond area sizes. For the 185,000 interpretation curve, SEDS results under-interpreted the disbond area by approximately $10 \%$.

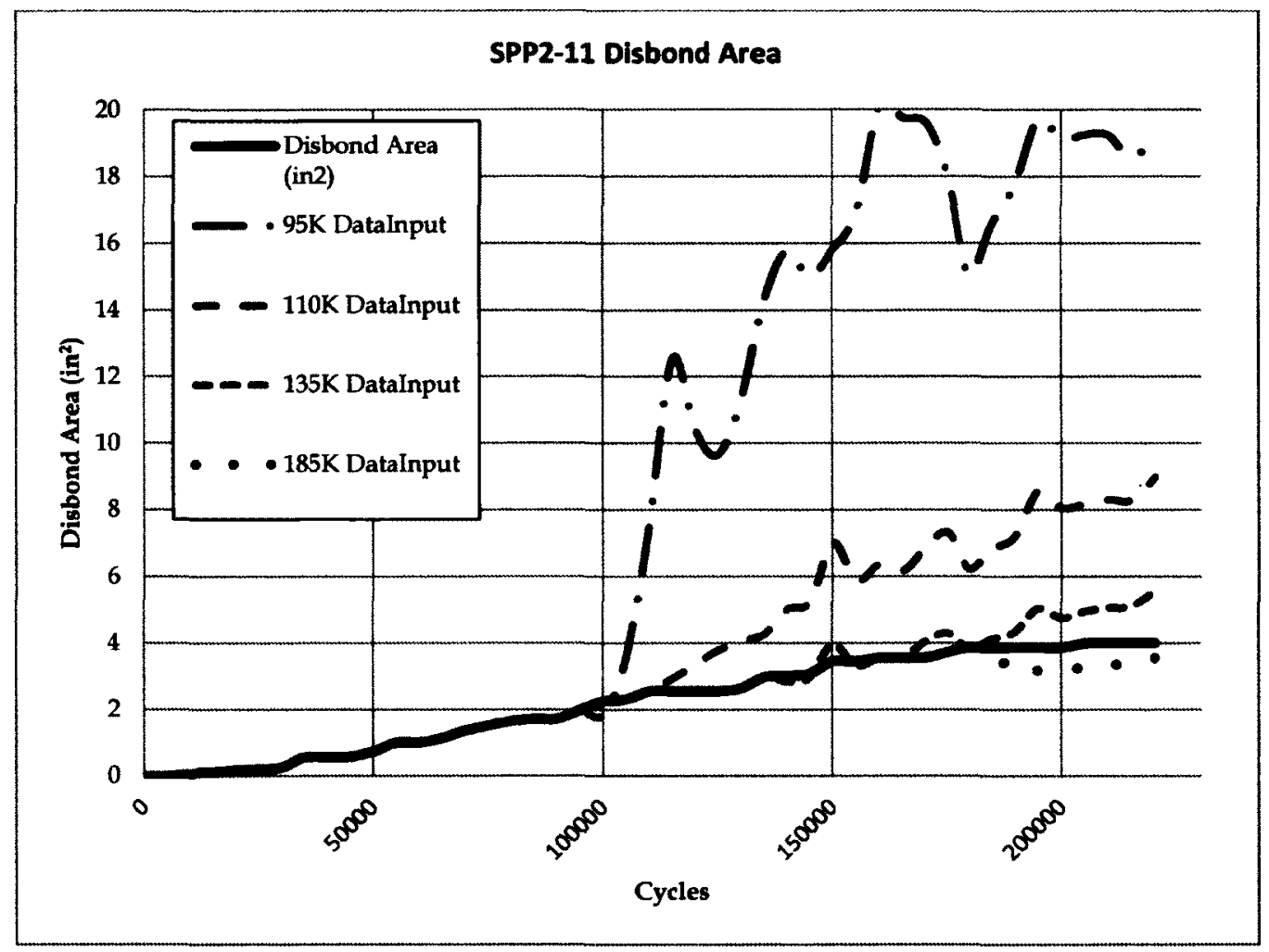

Figure 124: Coupon SPP2-11 Semi-Empirical Interpretations

Coupons SSP2-13 and SPP2-14 were subject to both crack growth and disbond but each coupon utilized different sensor configurations. SEDS analyses were carried out for both coupons; however, only the disbond area was quantified using the SEDS utility. The SEDS utility interpretation curves as well as the actual disbond area for coupon SPP2-13 are plotted within Figure 125 while the results for coupon SPP2-14 are plotted within Figure 126 . For both coupons, increasing the number of known disbond area inputs resulted in an increase of accuracy for disbond area interpretations. However, all SPP2-13 SEDS curves as shown in Figure 125 indicated disbond area that is significantly larger than that of the actual measured value and 
much larger than the area of the patch. No matter the number of known disbond area inputs, interpretations were inadequate in the determination of disbond area to within $1000 \%$ of the actual measured disbond area.

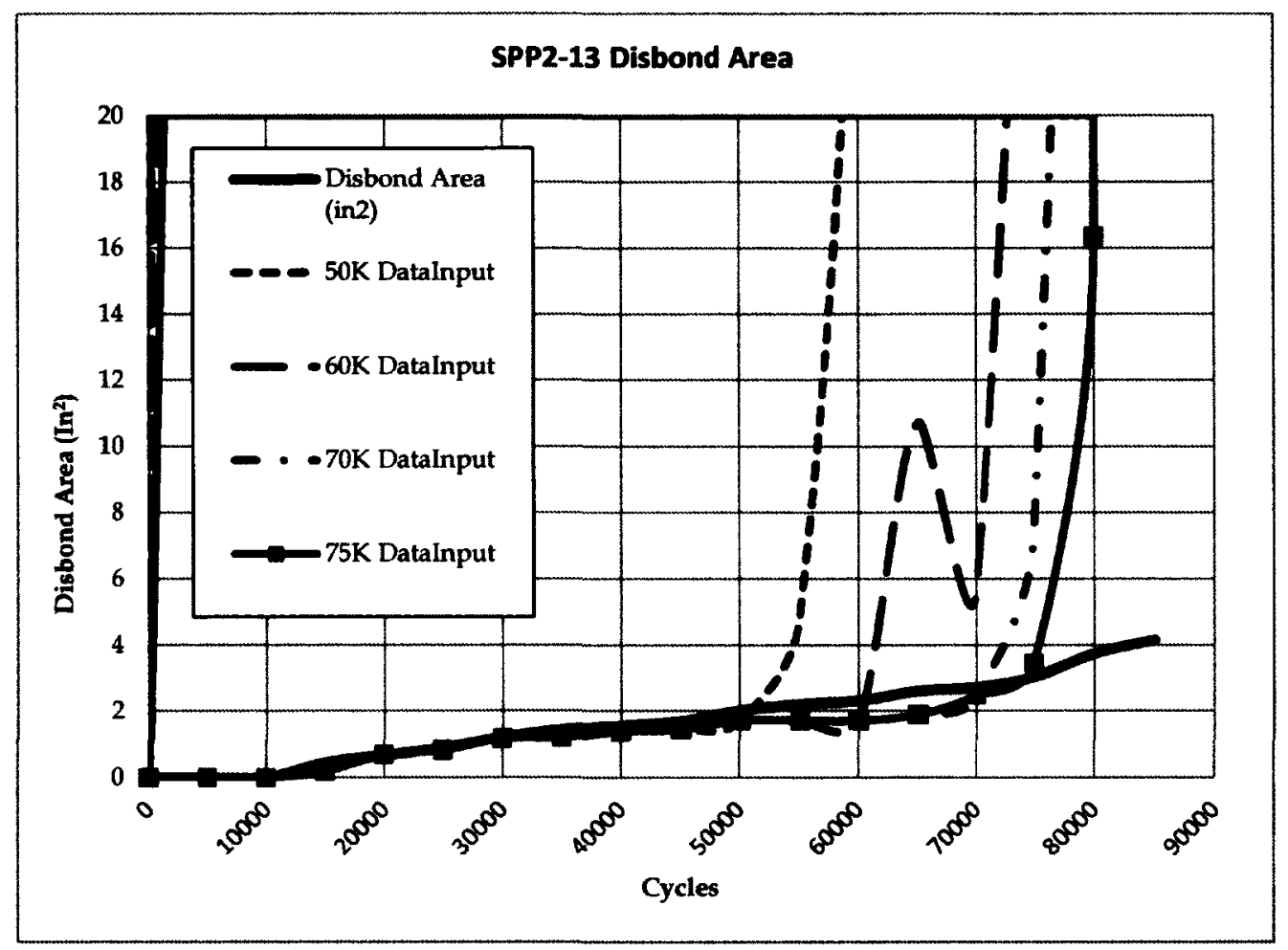

Figure 125: Semi-Empirical Interpretations for Coupon SPP2-13

Coupon SPP2-14 was equipped with 6 sensors as opposed to the 10 sensor configuration which was used on coupons SPP2-13. The SEDS utility was used to determine the disbond area size. Multiple SEDS analyses were computed, using 15 (50,000 cycle curve), 19 (70,000 cycle curve), 21 ( 80,000 cycle curve), $22(85,000$ cycle curve) and $25(100,000$ cycle curve) data point inputs as shown in Figure 126. Of all the SEDS curves, only those which had disbond area data points greater than 22 showed less than $200 \%$ over-interpretations of the disbond area. SEDS results utilizing less than 22 data point inputs lead to large interpretations exceeding $1000 \%$ of the actual disbond area. For disbond area data inputs greater than 25, the SEDS utility underinterpreted the disbond area. 


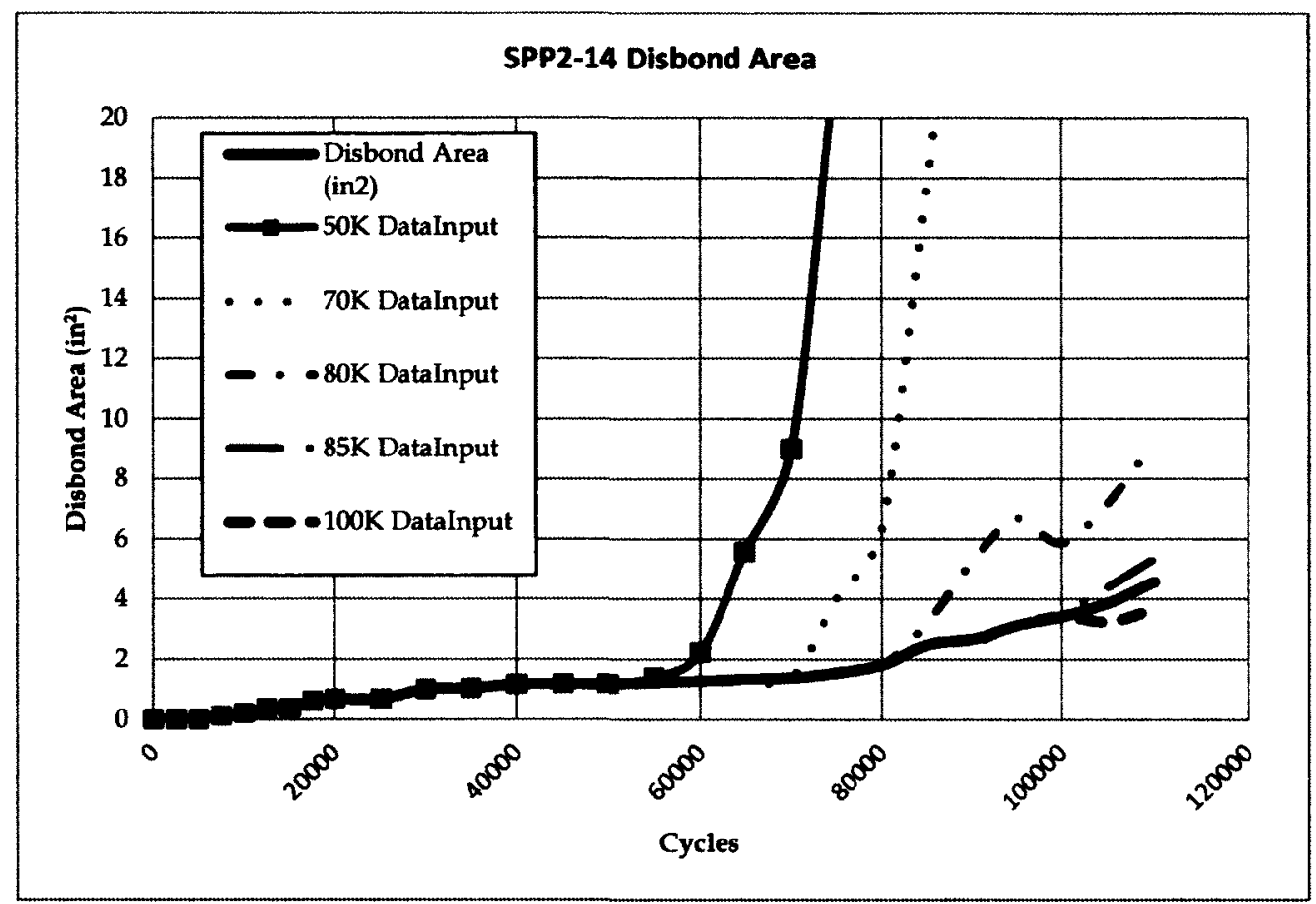

Figure 126: Semi-Empirical Interpretations for Coupon SPP2-14

Coupon SPP2-18 was identical to coupon SPP2-14; the coupon experienced the same failure and was equipped with an identical sensor configuration. Results for SPP2-18 were very similar to those of SPP2-14 and therefore SEDS graphs have not been presented within this chapter as they do not add additional information for the evaluation of the AU SHM system.

\subsection{Composite-to-Composite (Set 3)}

Coupons of Set 3 were designed for the evaluation of multiple SHM systems for the detection and quantification of disbond between composite components. This coupon set was intended to disbond under static loading; however, during testing it was found that the coupons failed within the substrate near the tip of the patch. To induce disbond, a fatigue based approach was utilized. These coupons were initially fatigued at a maximum load of $1,500 \mathrm{lb}$ and a minimum 
load of $150 \mathrm{lb}$. Under these conditions, the failure criteria was set such that $50 \%$ of the patch was allowed to disbond.

Coupons of Set 3 were of symmetric design and had two identical patches, one on either side of the substrate. Symmetry of the coupons allowed for the evaluation of multiple SHM systems; each system mounted on a single patch. Three SHM systems were evaluated using these coupons: Acousto-Ultrasonic (AU), Capacitance Disbond Detection Technique (CDDT) and Surface Mountable Crack Detection System (SMCDS). Each of the SHM systems was evaluated independently and results for which are presented within the following sections.

\subsubsection{Apparatus Setup}

Coupons of Set 3 were tested under cyclic conditions with loads between 1,500 lb and $150 \mathrm{lb}$ which correlate to 9375 PSI and 937.5 PSI, respectively. Inspection of coupon health for this set was executed through the use of AU, CDDT and SMCDS SHM systems and visual inspection.

Each of the tested coupons was monitored using two different SHM techniques and one NDE technique. All coupons were equipped with the AU sensors on one side and either CDDT sensors or Surface Mountable Crack Sensors (SMCS) on the opposing side. Initially, pulse thermography images were taken during analysis as the NDE technique. However, this approach was later changed as the system became unavailable. A visual inspection was implemented in place of the pulse thermography system to estimate disbond area throughout the coupon life cycle. Estimations of disbonded area were based on the outlining adhesive cracking on either side of the patch as shown in Figure $127(b)$. By drawing a connecting line between both cracks, a triangular area was formed as shown in Figure 127(c). The enclosed area was considered the estimated disbonded area. 


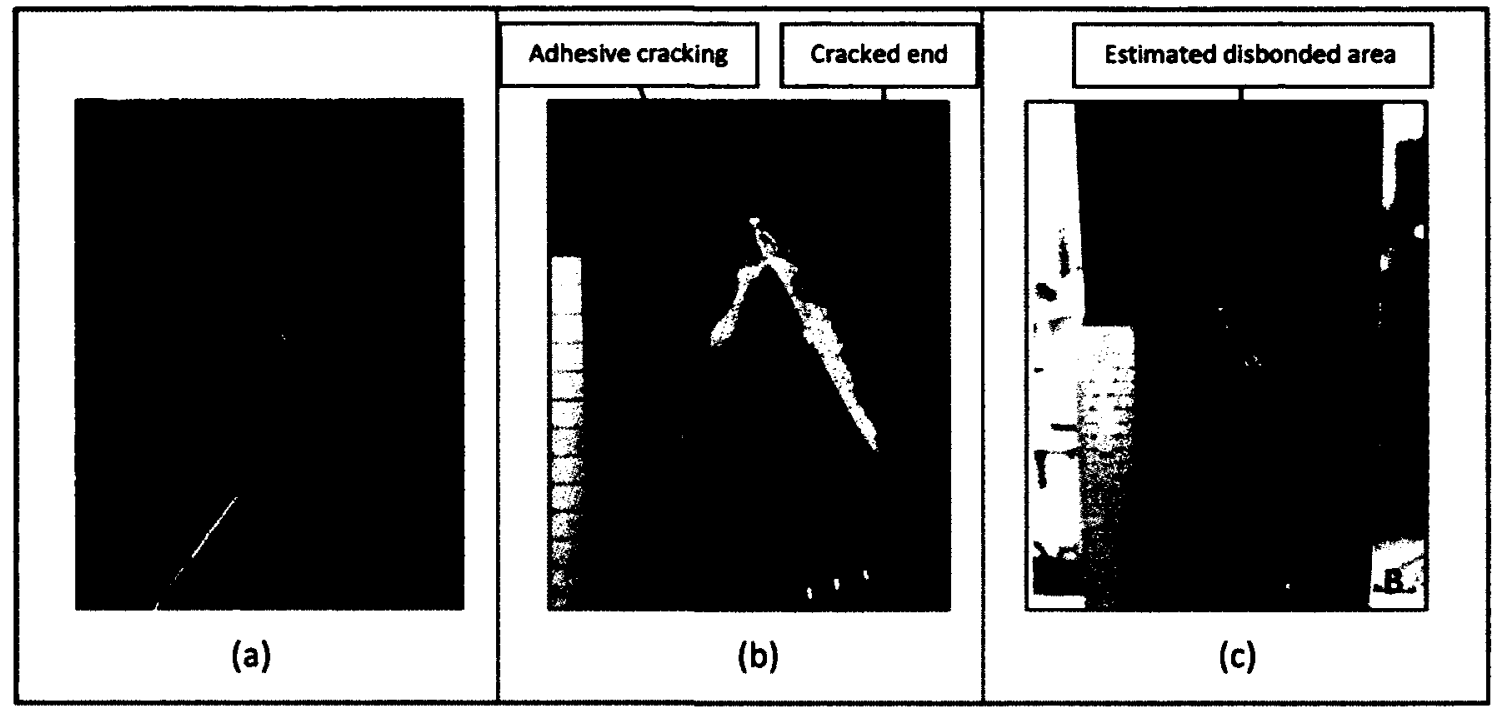

Figure 127: Disbond Area Estimations: (a) Coupon, (b) Adhesive cracking and (c) Estimated area

Coupons equipped with the two SHM systems were cycled at a frequency of $5 \mathrm{~Hz}$ and after every 5,000 cycles, the test frame was paused and data acquisition scans using the AU SHM systems were gathered and either the a capacitance reading was taken or a SMCS were checked for continuity. This process was repeated until the number of cycles reached 50,000. After 50,000 cycles data collection was carried out every 10,000 cycles up to 100,000 cycles after which data was collected every 25,000 cycles, this was done as disbond rates decreased as the disbond area increased.

\subsubsection{Results}

A total of five coupons were tested as part of Set 3. All coupons were of boron patch bonded to carbon fibre substrate design. All coupons of this set were tested utilizing the same procedure. Throughout the course of this research, disbond area measurements as obtained using the visual inspection were utilized to validate results which were obtained using all SHM systems. 
Two sensor configurations were used during testing: LW-1 and LW-2. LW-1 was comprised of sensors bonded to both the boron patch and the carbon fibre substrate as shown in Figure 128. Sensors bonded to the substrate were found to disbond when fatigued between $1,500 \mathrm{lb}$ and $150 \mathrm{lb}$, as shown in Figure 128 magnification $A$. Disbond of the sensors initiated directly underneath the Lead Zirconate Titanate (PZT) wafer as shown in Figure 128 magnification A. Disbond was a result of high strain within the substrate, which exceeded that of the strain of the adhesive used. Disbond of sensors led to both poor signal transmission and reception. Changes in the signal due to sensor adhesion failure could no longer be compared to the benchmark signal data. Figure 129 shows disbond of sensors and its effect on signal transmission. Figure 129(a) shows signal transmission path of sensors 2 and 5. Figure 129(b) shows two signals each originating from sensor 2 and received by sensor 5; the benchmark signal data was taken prior to sensor disbond while the current signal data was taken after disbond at 20,000 cycles. To overcome the problem of sensor disbond, LW-2 sensor configuration was developed and implemented. Observations of LW-1 sensors indicated that only sensors found on the substrate were subject to disbond; for that reason LW-2 only utilized sensors on the patch. Figure 130 shows the sensor configuration LW-2. Sensors bonded to the patch of the coupon did not experience high strains and therefore did not disbond. Both $L W 1$ and $L W-2$ were analysed using the DPI and the SEDS utilities as presented within the following sections. 


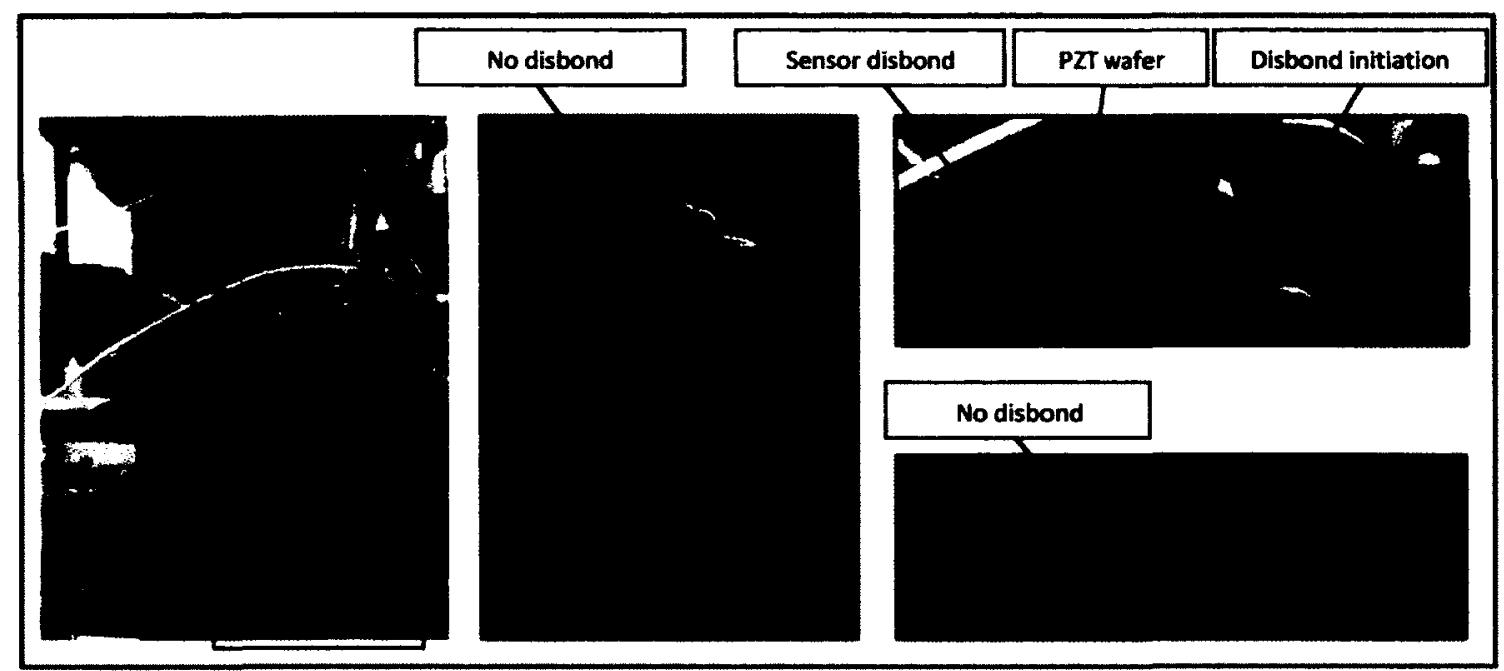

Figure 128: Sensor disbond

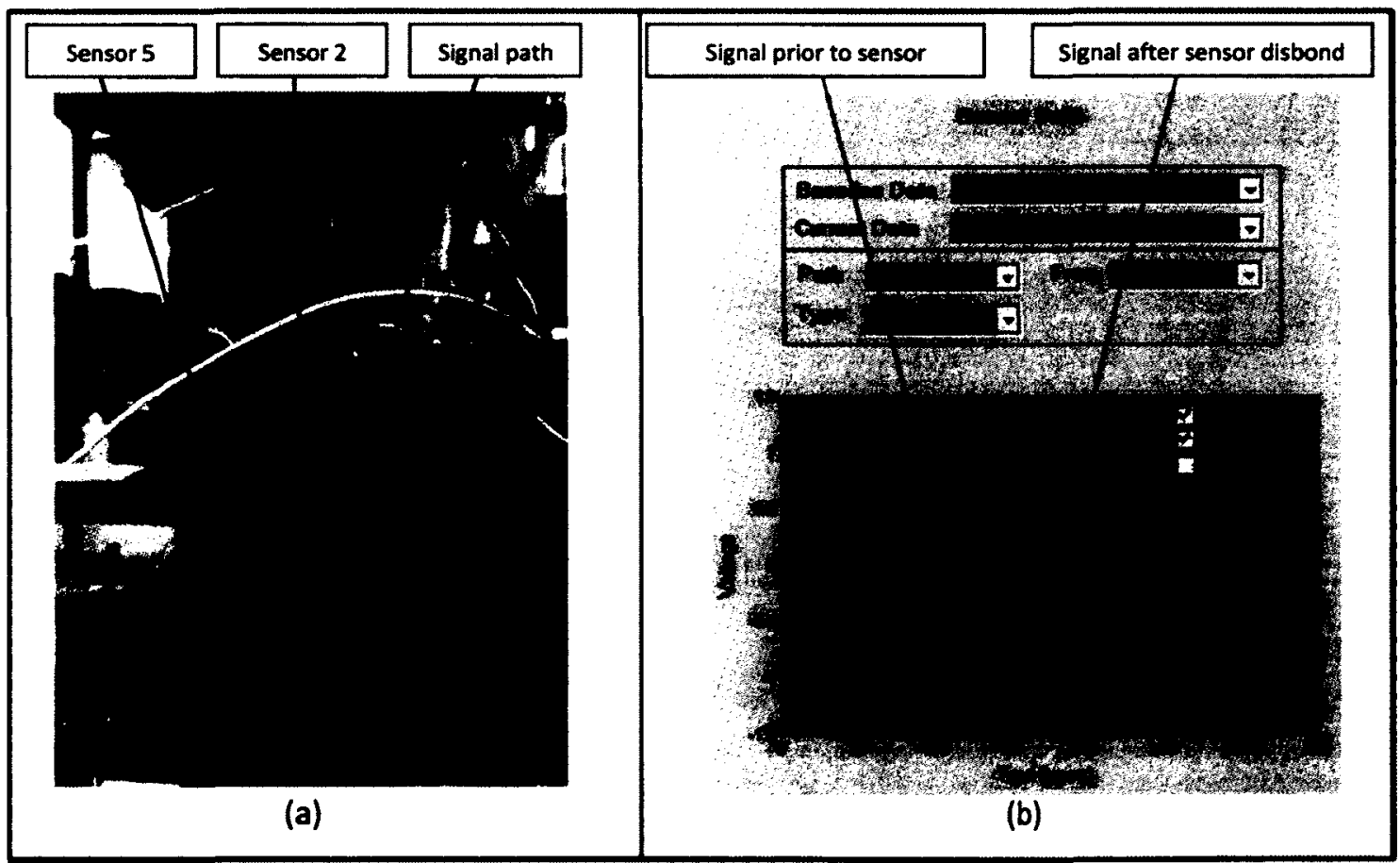

Figure 129: Signal Change due to Sensor Sisbond: (a) Signal path and (b) Signal change due to sensor disbond 


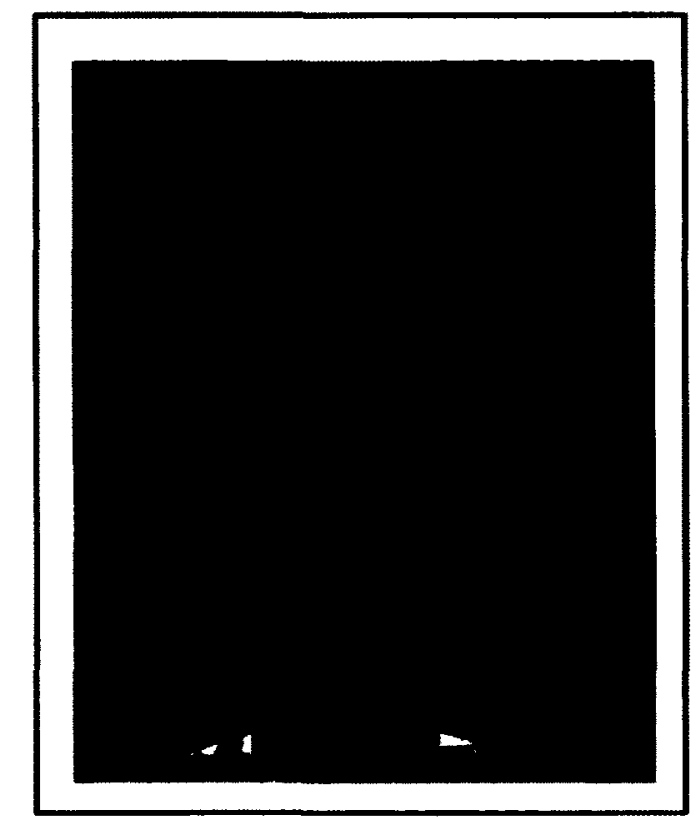

Figure 130: Sensor Configuration Modification

\subsubsection{Direct Path Image (DPI) utility results}

There were five coupons analyzed using the DPI utility: three of which used LW- 1 and the other two used LW-2 sensor configurations. SPP3-03, SPP3-04 and SP3-16 utilized LW-1 while SPP3-4a and SPP3-12 utilized LW-2. Results for coupons SPP3-03, SPP3-04 and SP3-16 (LW-1 configuration) were very similar to each other and therefore only DPI utility results for SPP3-04 are presented within this chapter. Similarly for LW-2, both coupons yielded similar results and therefore only DPI utility results for SPP3-12 are presented within this chapter.

Coupon SPP2-04 was constructed of a boron patch and a carbon fibre substrate. The coupon was instrumented with $8 \mathrm{AU}$ sensors which were wired to the ScanGenie ${ }^{\bullet}$ system. The 8 sensors allowed for many scanning paths; analysis using the DPI utility was carried out using all paths. Multi-zone analysis was not carried out on these coupons, as sensor disbond had occurred. Figure 131 shows the DPI utility image results for $20,000,50,000,80,000,100,000$ and 150,000 cycles and their associated visual inspection images. Analysis of the DPI utility images indicates 
the presence of disbond in as early as 20,000 cycles. However, when examining the visual inspection results for 20,000 cycles, it is evident that the patch is fully bonded to the substrate. Disbond progression as shown from left to right within Figure 131 is poorly indicated using the DPI utility images as the highlighted areas within the images remain unchanged as damage grows. The indication of damage on all areas of the patch is a result of two simultaneous actions: patch disbond and sensor disbond. For all these coupons, damage as indicated by signal change through the DPI utility images is dominated by the sensor disbond and not patch disbond. 


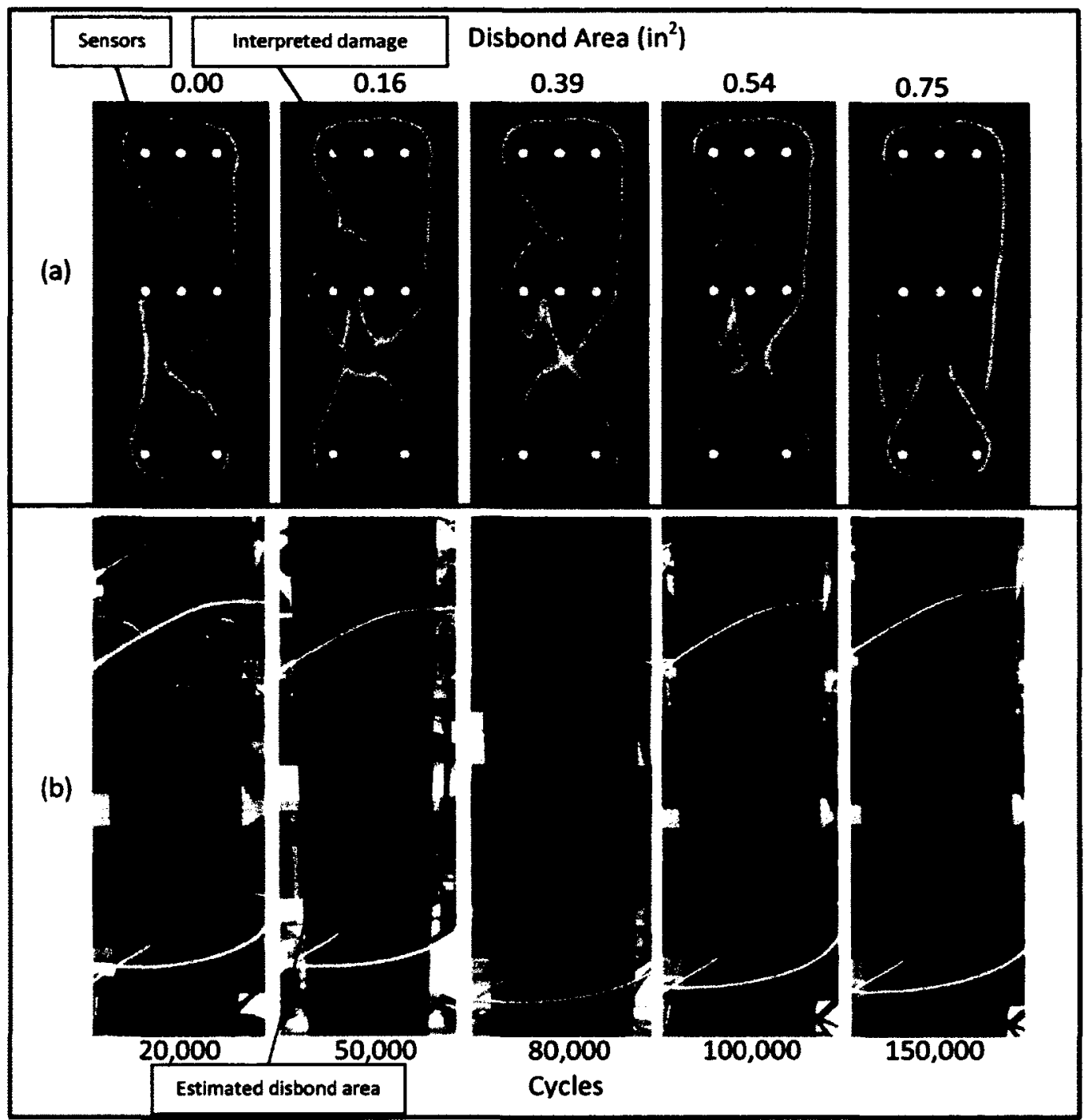

Figure 131: Coupon SPP3-04: (a) DPI utility results at 05 threshold and (b) Estimated disbond areas

Coupon SPP2-12 was identical to that of SPP3-04 with the exception of sensor configuration.

The coupon was instrumented using the LW-2 configuration which utilized 4 AU sensors. The 4 sensors allow for many scanning paths; however, unlike all other configurations used throughout this study, this configuration allowed for only single zone analysis. Figure 132 shows the DPI utility image results for $20,000,50,000,80,000,110,000$ and 200,000 cycles and their associated visual inspection images. Analysis of the DPI utility images indicates the presence of disbond immediately after initiation as shown in both the DPI utility and visual inspection 
images. Disbond progression was also observed through the examination of the images from left to right. The highlighted area on the DPI utility image increased in both intensity and sized as the actual disbond area enlarged. This indicated good correlation between the actual disbond area and damage indicated using DPI utility.

After examining all 5 coupons using the DPI utility for damage identification, the SEDS was applied to determine the size of such damage; this is presented in the following section.

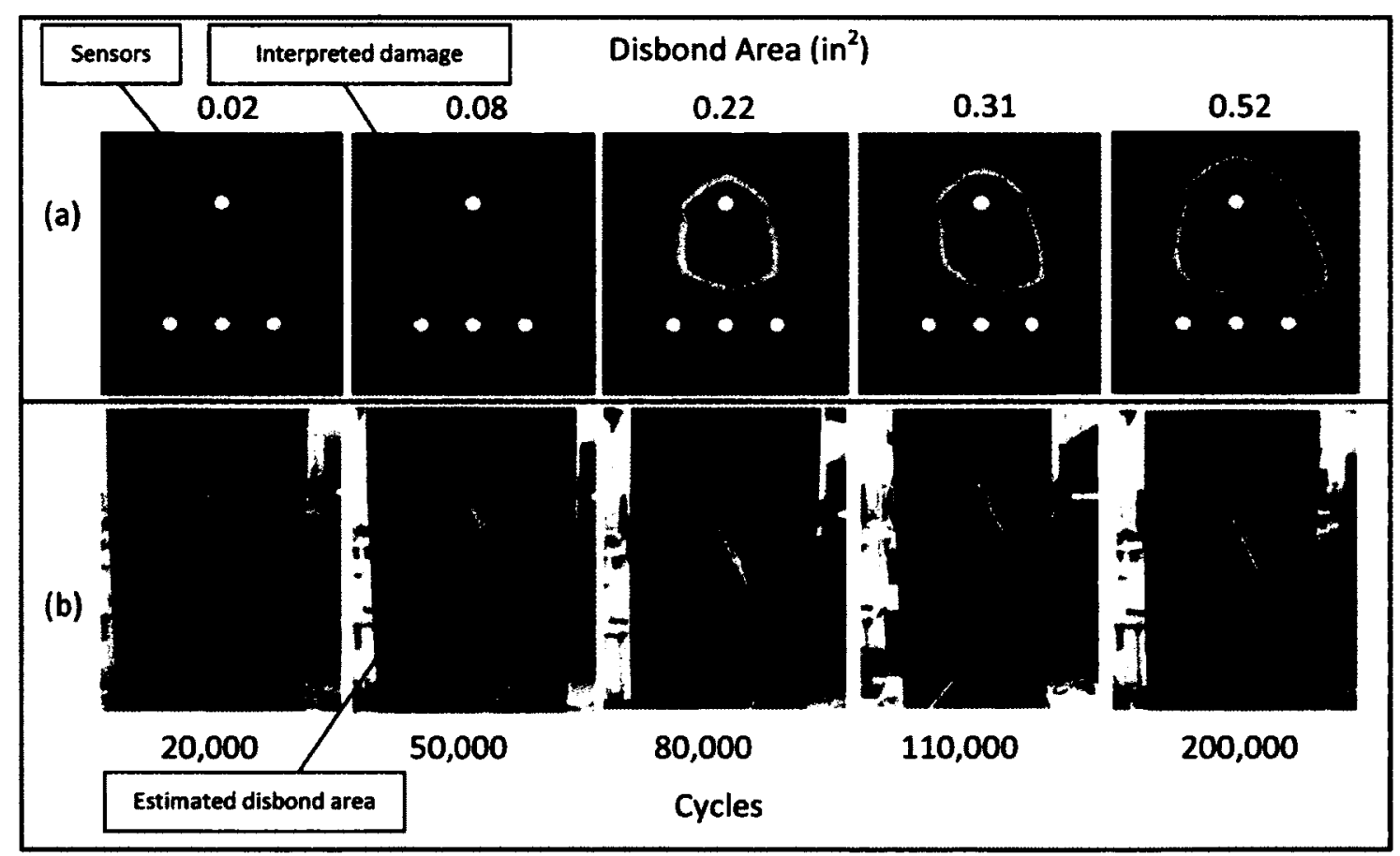

Figure 132: Coupon SPP3-12: (a) DPI utility results at 0.5 threshold and (b) Estimated disbond areas

\subsubsection{Semi Empirical Damage Sizing (SEDS) results}

The SEDS utility is one of the two utilities found within the SmartPatch application and its purpose was to determine disbond area at all inspection points throughout the coupon life cycle. The SEDS utility is able to analyze signal data and provides an "interpreted" damage size; in this case, disbond area. Interpreted disbond areas were a result of Damage Indices (DI), 
which were described in more detail within section 2.2.3.2. Damage Indices are obtained by creating a Bspace file and by providing known disbond areas for the first few inspections to the application. For SPP3-03, SPP3-04, SPP3-16, SPP3-4a and SPP3-12 approximately $40 \%$ of all collected signal data was assigned to the Bspace file. Multiple analyses were executed for each coupon, each with a different number of known disbond area inputs. Coupons SPP3-03, SPP3-04 and SPP3-16 were equipped with LW-1 configuration while SPP3-4a and SPP3-12 were equipped with LW-2. Results for all coupons utilizing $L W-1$ sensor configuration were similar and therefore only one set of results is present below.

Coupon SPP3-04 was equipped with the LW-1 sensor configuration and was analysed under multiple input conditions using the SEDS utility, each analysis with different known disbond area inputs. Analyses for the interpretation of disbond area were executed with as little as 12 and as high as 21 known disbond area inputs. In total, there were 23 signal data files gathered for this coupon and the SEDS utility analysed all signal data against the Bspace to determine disbond areas which were not provided to the software. Figure 133 shows disbond area interpretations for analysis with 12 (60,000 cycle curve), 14 (80,000 cycle curve), 16 (100,000 cycle curve), 18 (120,000 cycle curve) and 21 (150,000 cycle curve) disbond area data point inputs. In addition to the analysis curves, the actual disbond area curve is also plotted within Figure 133. While examining each of the curves produced by the SEDS utility within Figure 133, it is evident that none of the interpretation produced values which are close to that of the actual disbond area. Increase of the number of disbond area inputs resulted in over-predictions of the disbond area. The over-interpretation of disbond area was attributed to disbond of both the patch and the sensors; primarily dominated by sensor disbond. 


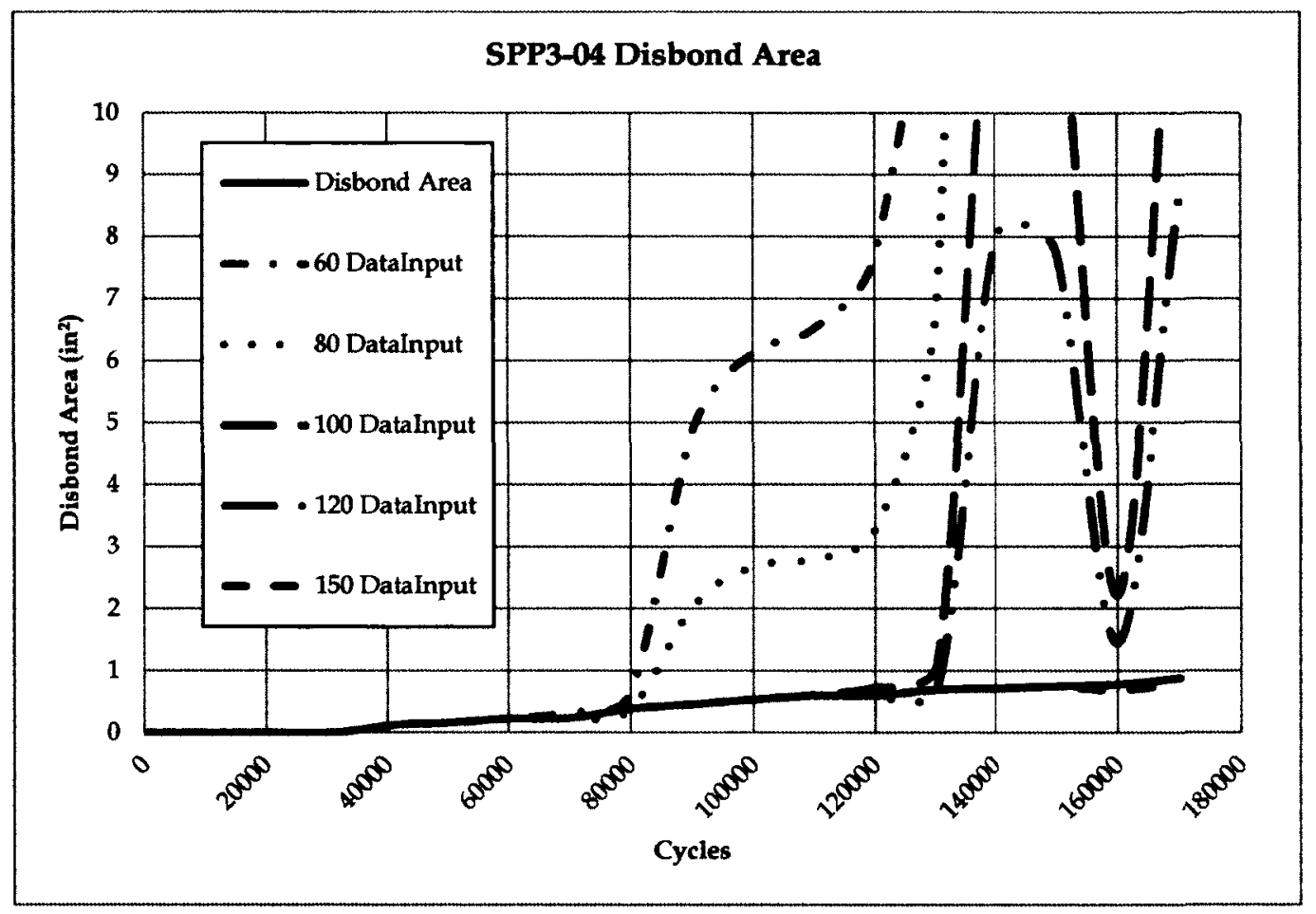

Figure 133: Semi-Empirical Interpretations for Coupon SPP3-04

Coupons SPP3-4a as well as SPP3-12 were both equipped with LW-2 sensor configurations. Identical coupons with identical sensor configurations have yielded identical results for all previously tested coupons; however, for coupons SPP3-4a and SPP3-12 this was not the case. SEDS utility results for both coupons exhibited two distinct curve patterns as shown in Figure 134 and Figure 135. Figure 134 shows SEDS utility results as well as actual disbond area sizes for coupon SPP3-4a. SEDS utility analyses were executed using $9(40,000$ cycle curve), $12(60,000$ cycle curve), 14 (80,000 cycle curve), 16 (100,000 cycle curve) and 17 (150,000 cycle curve) disbond area input data points. Increasing the number of inputted data points increased the accuracy of disbond area interpretations. However, examination of all SEDS utility curves revealed that all interpretations were represented by exponentials. Exponential approximations led to an over-interpretation for all disbonded areas. 12 and 17 data input points yielded disbond area interpretations within $1000 \%$ and $100 \%$ accuracy, respectively. Coupon SPP3-12 is identical to SPP3-4a and SEDS utility results are shown in Figure 135. SEDS utility analyses were 
executed using 8 (70,000 cycle curve), 10 (90,000 cycle curve), 12 (110,000 cycle curve), 14 (130,000 cycle curve) and 15 (140,000 cycle curve) disbond area input data points. For all curves with data input points that are greater than 10 , results of the interpreted disbond area were within $15 \%$ and $138 \%$ accuracy. The $15 \%$ result correlated to that of 15 data point inputs and $138 \%$ correlated to that of 9 data point inputs.

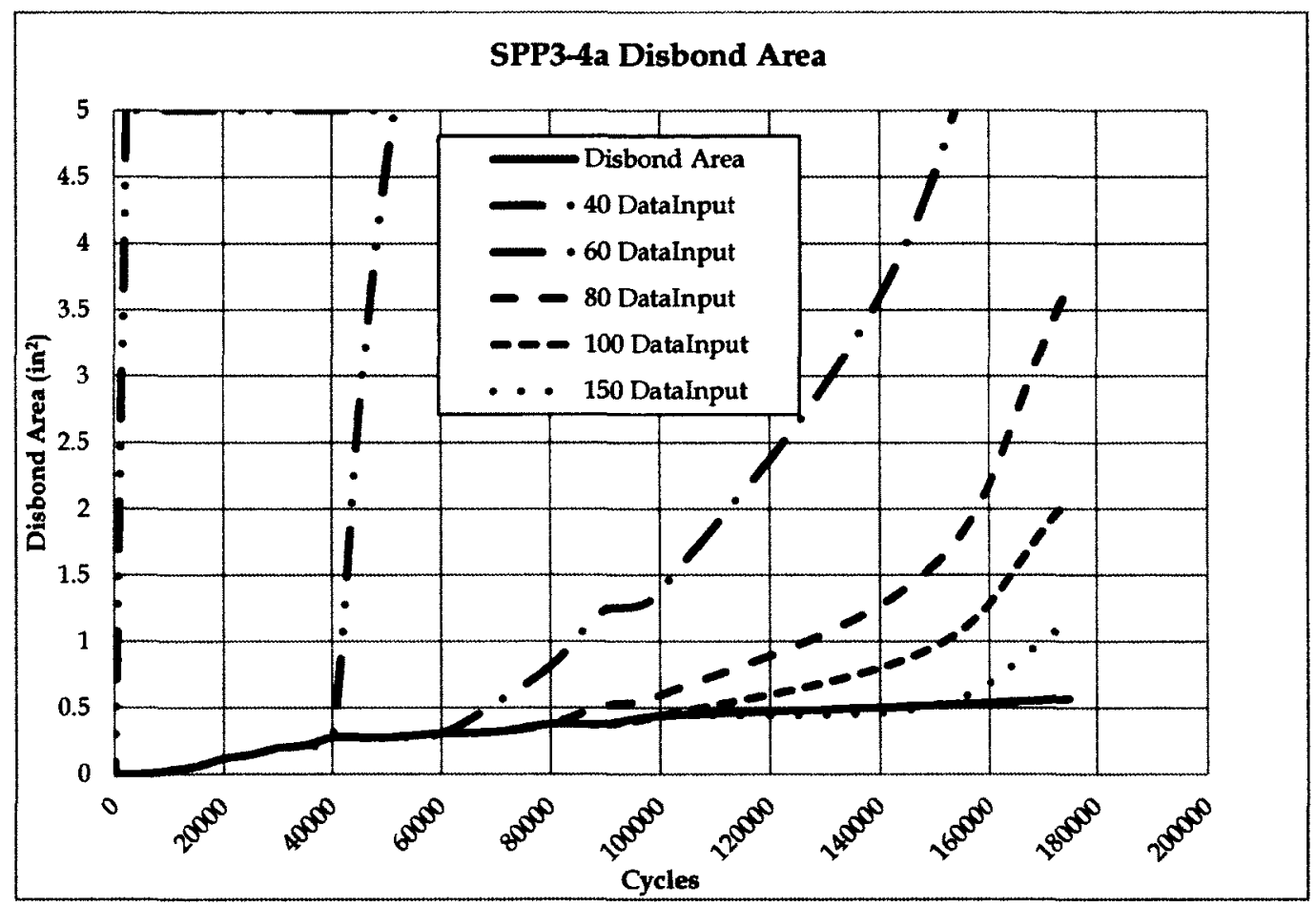

Figure 134: Semi-Empirical Interpretations for Coupon SPP3-4a 


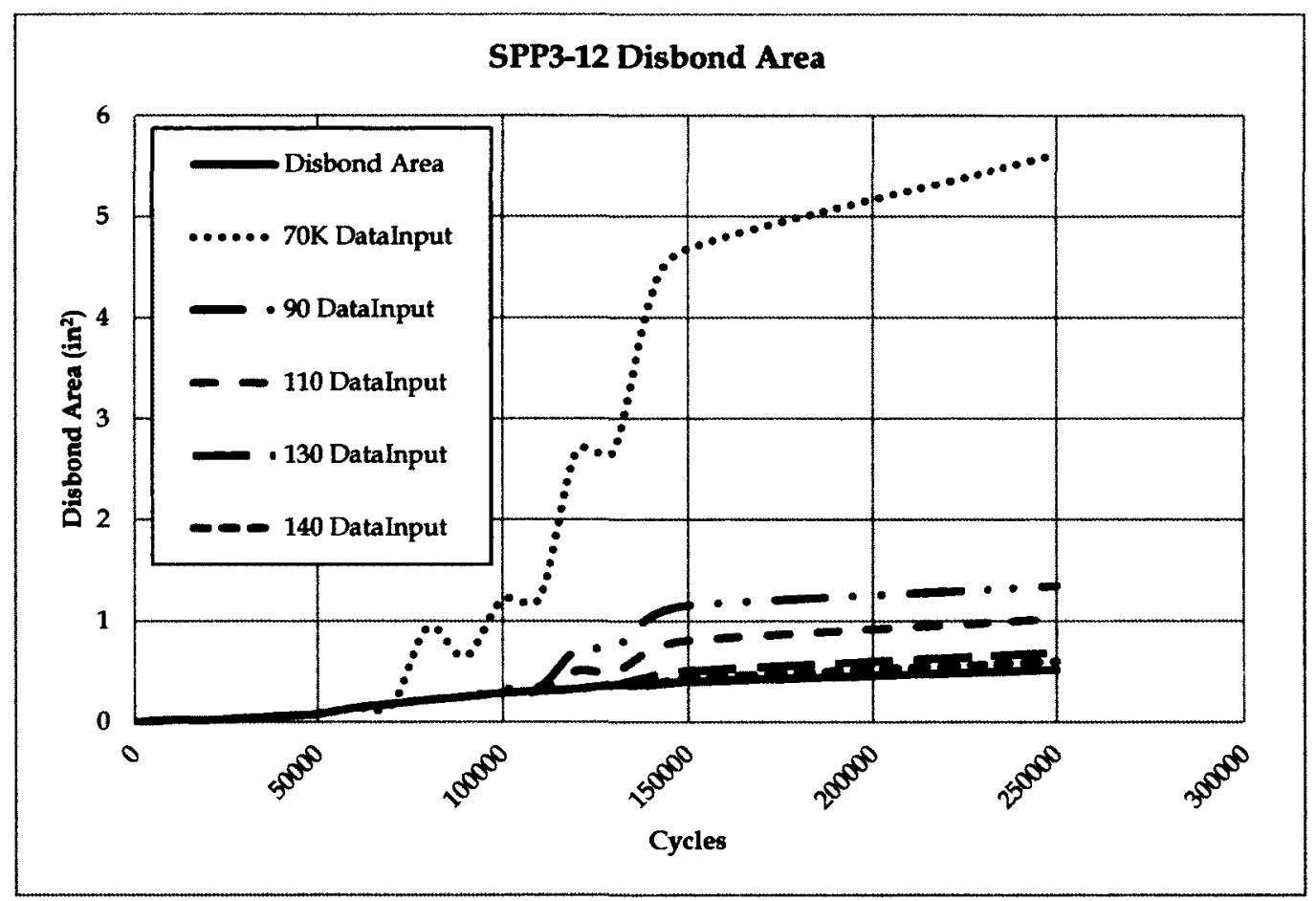

Figure 135: Semi-Empirical Interpretations for Coupon SPP3-12

\subsubsection{Capacitance Disbond Detection Technique (CDDT) results}

All tested coupons were instrumented with the AU sensor and either a CDDT sensor or SMCS. Of all the 5 tested coupons, SPP3-12 and SPP3-16 were equipped with a CDDT sensor for the detection and quantification of disbond. CDDT was used on these patches to determine if a correlation between change in disbond area and capacitance carrying capabilities existed for adhesive bonded joints. Both coupons showed similar results.

Capacitance measurements as well as visual inspections were carried out on coupon SPP3-16 and results are presented within Figure 136 . Figure 136 shows both the disbond area $\left(\mathrm{in}^{2}\right)$ and capacitance (nF) as a function of number of cycles. It is evident from the two curves in Figure 136 that a drop in capacitance could be correlated to an increase in disbond area. There were two points found on the capacitance curve that reflected an increase in capacitance. While an increase in capacitance should not have occurred, it may be correlated to temperature and 
humidity changes. It was observed that while using the thermography cameras, xenon flashes caused and increase in capacitance for approximately 30 seconds prior to returning to its original steady state value. In addition to the thermography flashes, capacitance increases were observed when readings were taken early in the day after no overnight testing. Both the increase of capacitance points shown in Figure 136 were attributed to recording capacitance values in the morning after resuming testing.

Coupon SPP3-12 was also analysed using CDDT and results for which are presented in Figure 137. Coupon SPP3-12 exhibited the same trends as those observed for coupon SPP3-16; increase in disbond area resulted in a decrease of capacitance. SPP3-12 was tested over the course of two days and therefore, an increase of capacitance was only observed once within the capacitance curve shown in Figure 137.

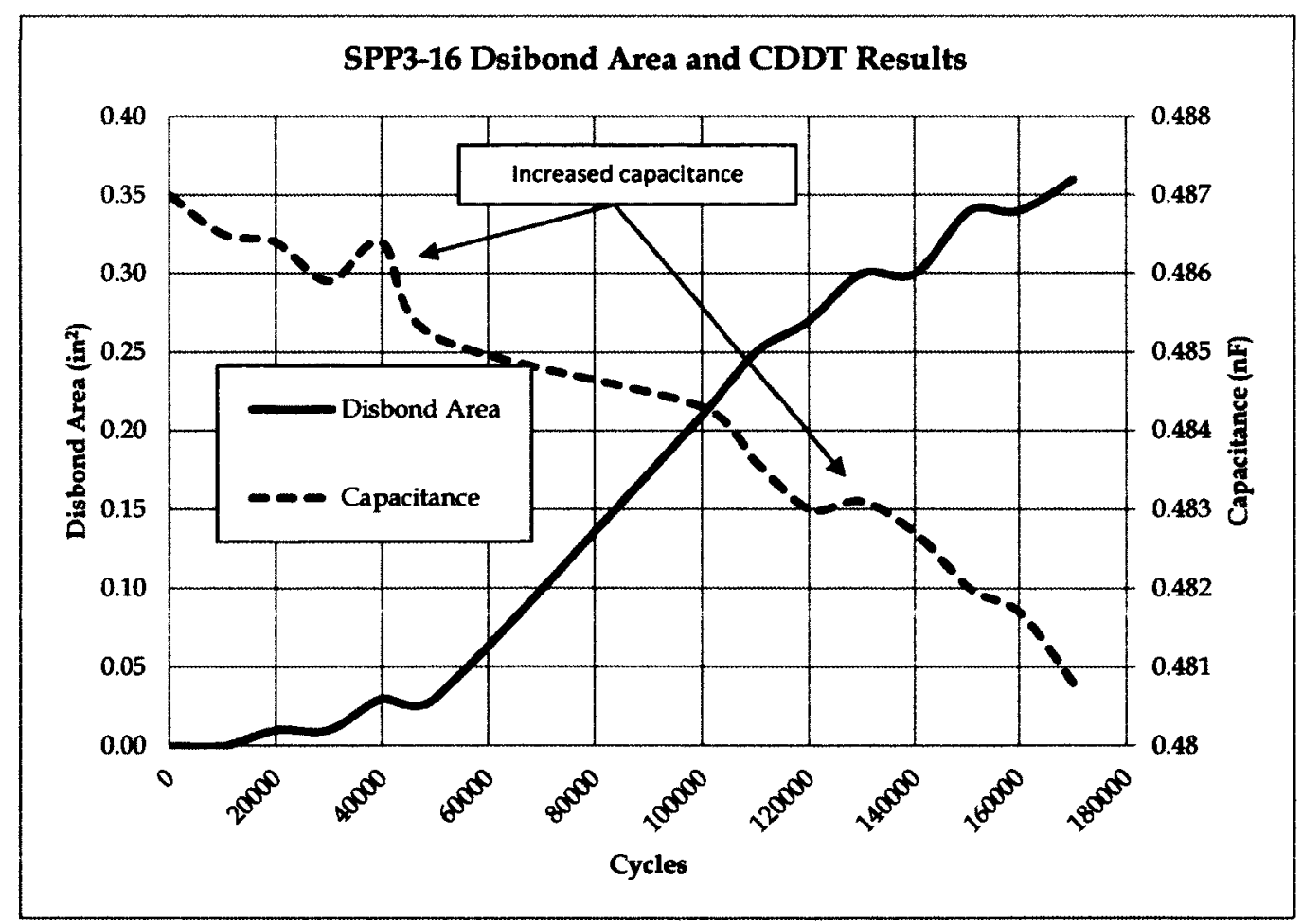

Figure 136: SPP3-16 Disbond Area Comparison to Capacitance Drop 


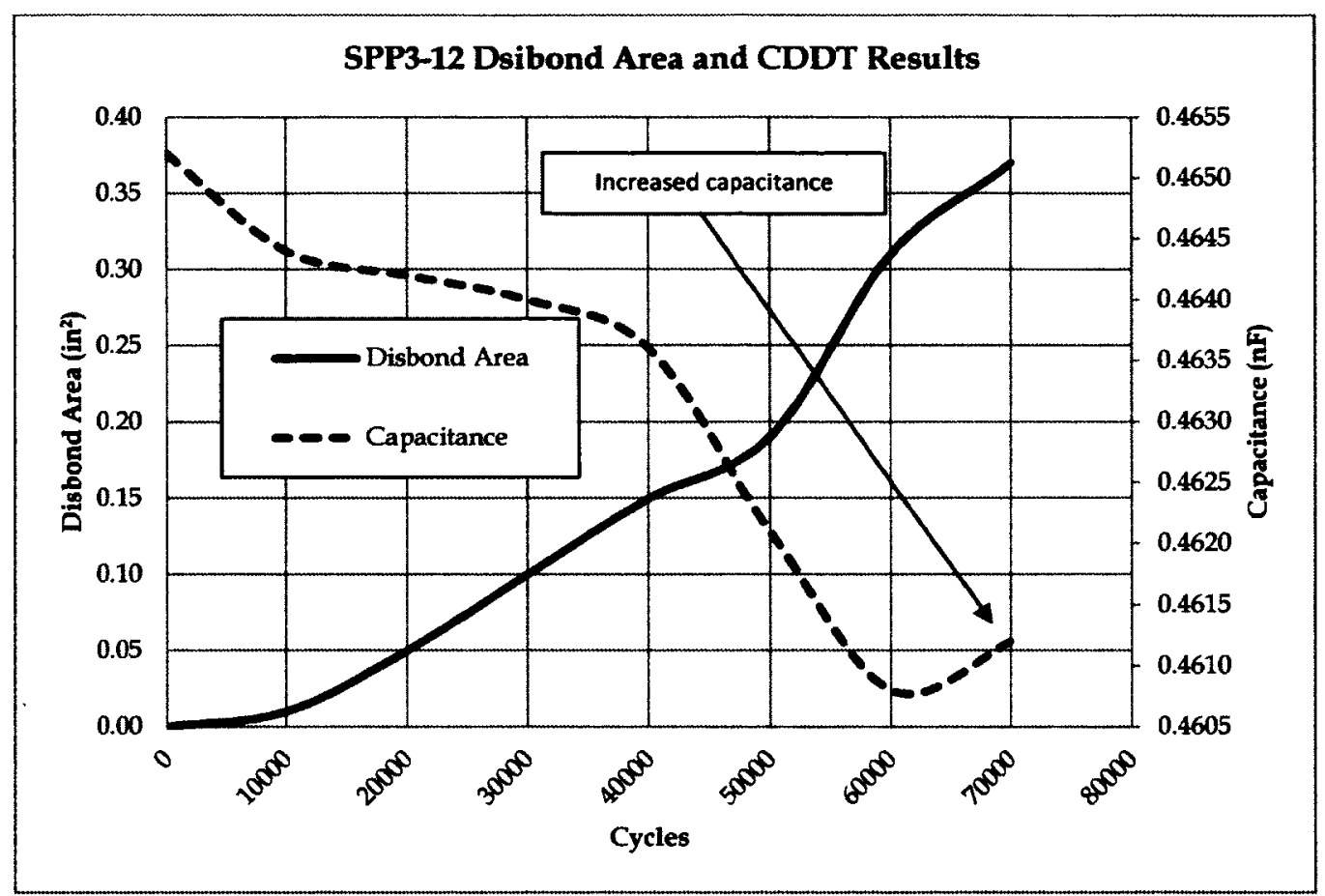

Figure 137: SPP3-12 Disbond Area Comparison to Capacitance Drop

Figure 138 and Figure 139 show the same results as Figure 136 and Figure 137; however, the disbond area was normalized as percentage of the overall patch area. This normalization allows for a quick depiction of the correlation found between the capacitance and disbond. With the exception of minor perturbations within both the disbond area and capacitance curves, both curves have a decreasing value which may be approximated using a linear regression. 


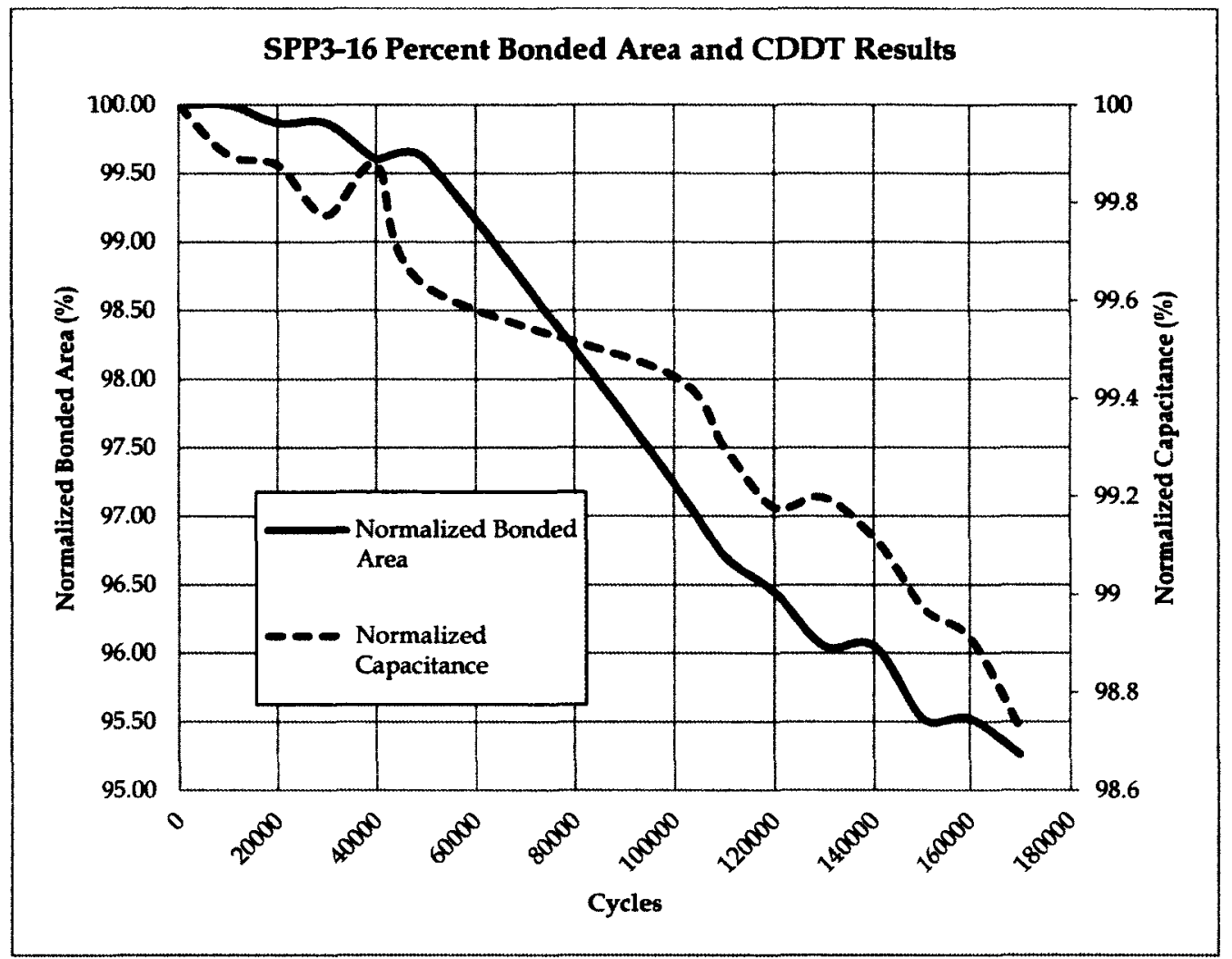

Figure 138: SPP3-16 Normalized Disbond Area Comparison to Capacitance Drop

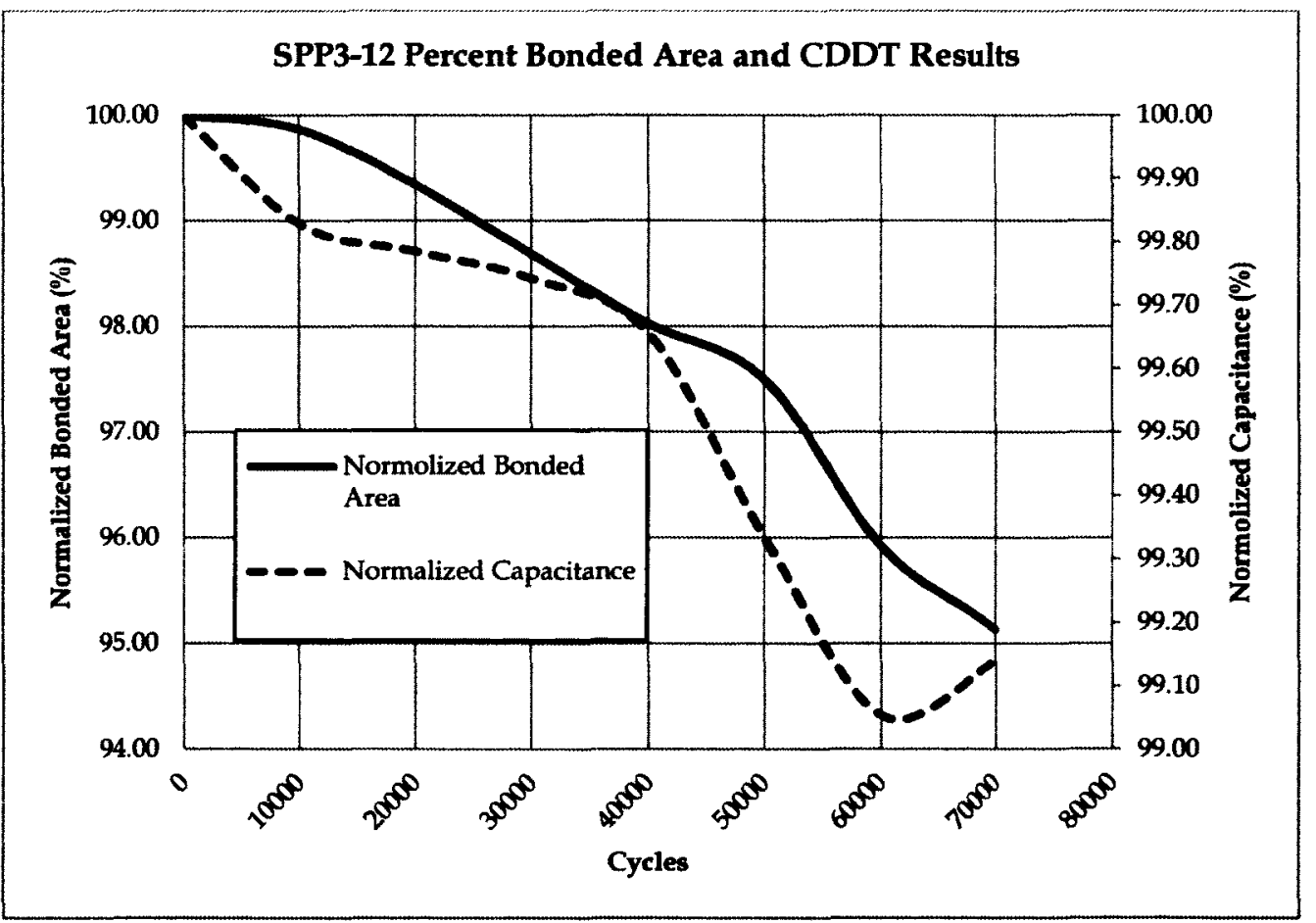

Figure 139: SPP3-12 Normalized Disbond Area Comparison to Capacitance Drop 
Based on the above results, this SHM technique provides value in the quantification of damage. Equations may be developed to model the change in capacitance with respect to disbond; however, these equations will be only applicable to this patch configuration. Changes in patch shape, temperature, humidity and ultra violet are amongst parameters that will both influence the equations as well as the reliability of this system.

\subsubsection{Surface Mountable Crack Detection System (SMCDS) results}

All tested coupons were instrumented with AU sensors and either a CDDT or SMCS. Of all the 5 tested coupons, SPP3-03, SPP3-04 and SPP3-4a were equipped with SMCS for the detection of disbond. Unlike both the AU and the CDDT SHM systems, SMCS does not quantify the disbond area size rather it indicates when cracks along the edge of the patch have reached a certain point. SMCS were only placed along strategic locations around the tip of the patch as shown in Figure 140. As cracks within the adhesive grew alongside the patch, certain SMCS were triggered (i.e. the circuit was broken). Analysis for all coupons instrumented using the SMCDS technique resulted in the same conclusions and therefore only results for one coupon are presented.

Coupon SPP3-04 was equipped with the SMCS as shown in both Figure 140 and Figure 141. Three SMCS were placed approximately 0.15 inches apart as shown in Figure 140 magnification A. During fatigue, cracks initiated within the adhesive, at the tip of the patch and propagated through the SMCS as shown in Figure 141. Crack growth through the SMCS resulted in an open electrical circuit during applied load and closed circuit during no load. It was also difficult to visually inspect SMCS cracking with no applied load as the circuit was closed and the SMCS is very reflective under lighting as shown in Figure 141. For that reason, the circuit was interrogated during fatigue, and upon the first open circuit indication, the circuit was assumed broken regardless of its future open/closed positions. At later stages within the lifecycle of the 
coupon, cracked areas within the conductive paint of the SMCS began to chip as shown in Figure 142; hence reducing the integrity of the sensor. The insulating layer which acts as a barrier between the conductive paint and the conductive carbon fibre was also found to crack under fatigue as shown in Figure 142. The insulating layer cracking would normally be problematic as it would cause the SMCS to short circuit. However, short circuiting was not problematic as cracking occurred well after the tripping of the SMCS.

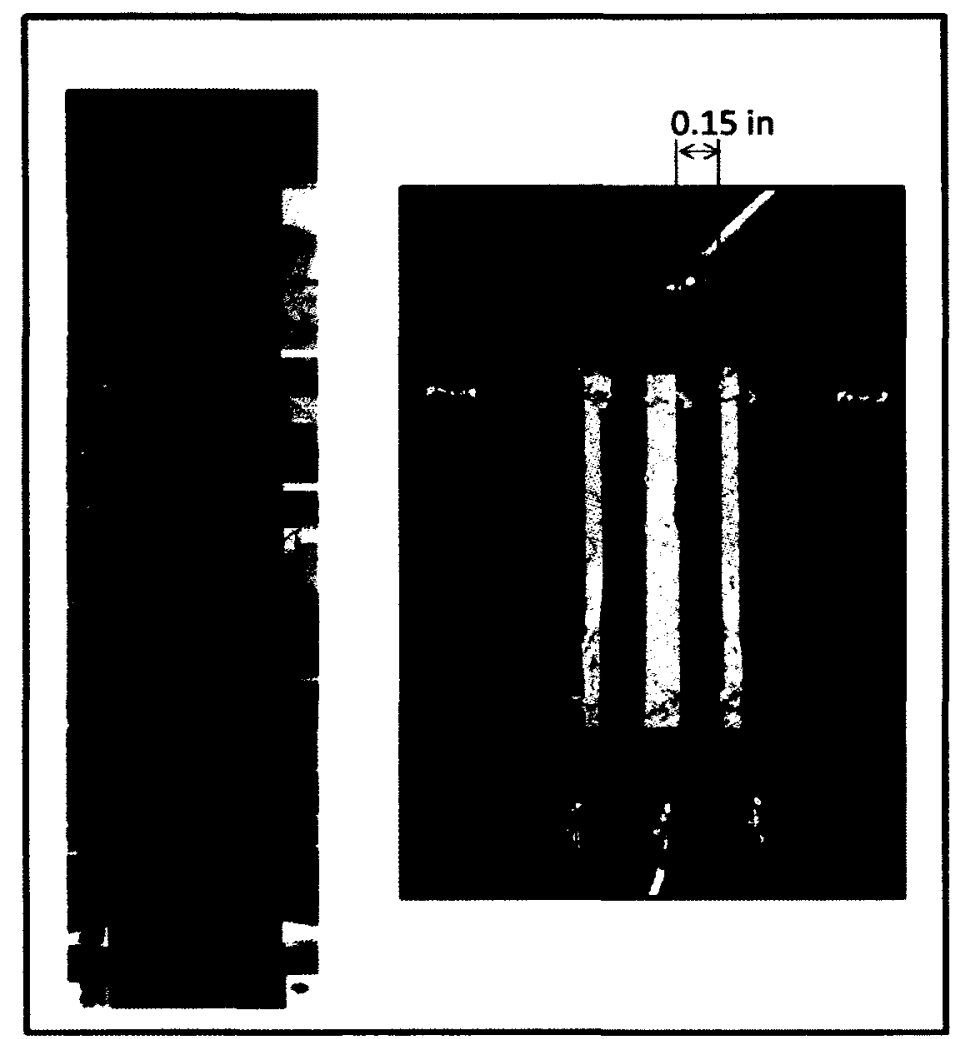

Figure 140: SPP3-04 SMCS Placement along Patch Edge 


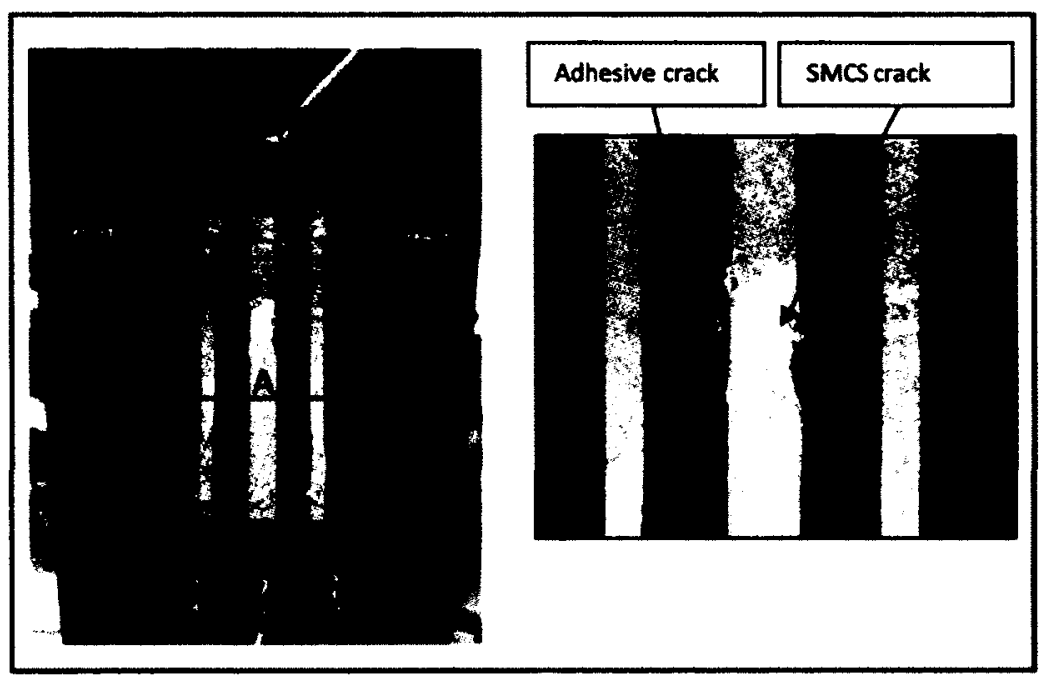

Figure 141: SPP3-04 SMCS Breakage

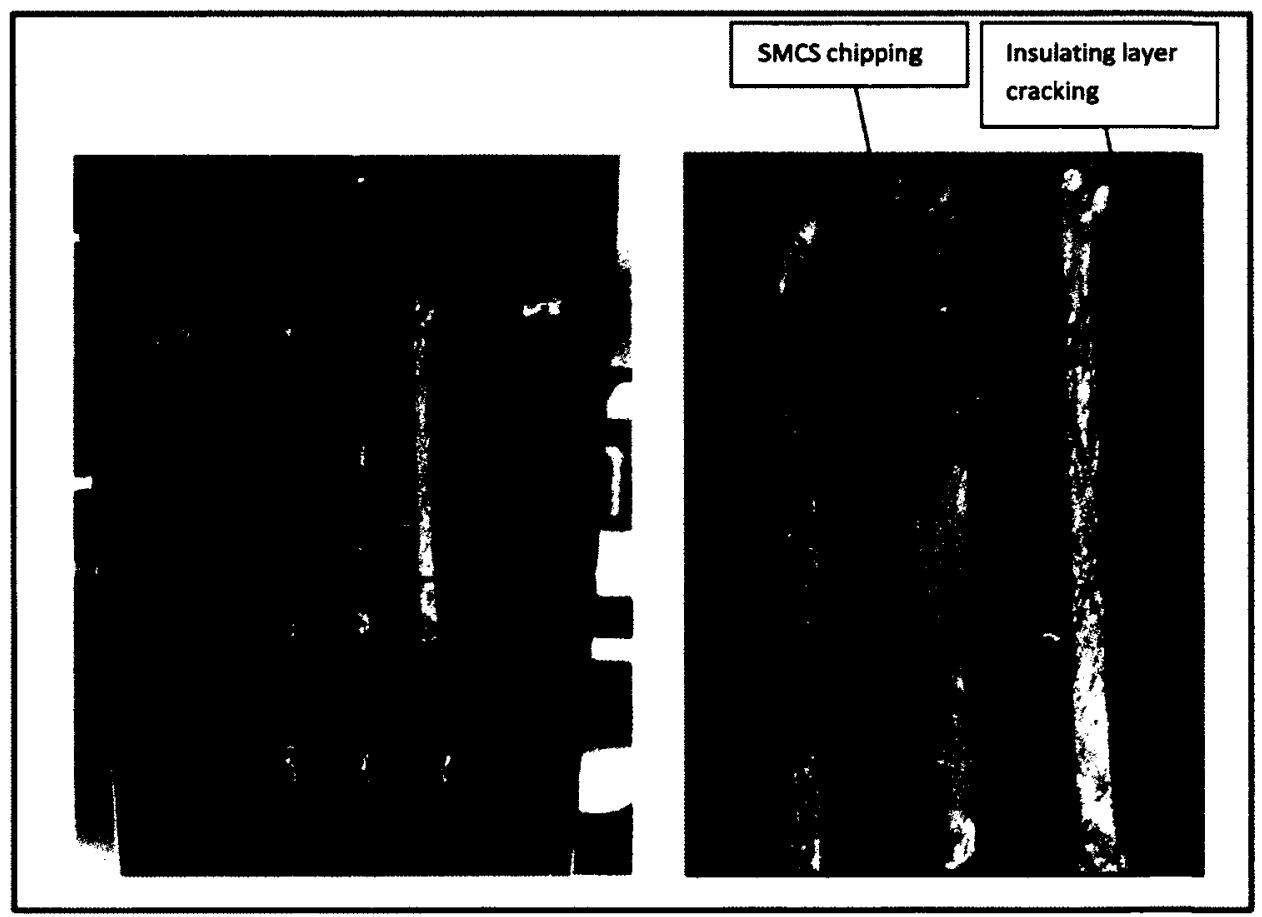

Figure 142: SPP3-4a SMCS Breakage at High Cycles

Based on the above results, this SHM technique provides value in the detection of adhesive cracking. Sensors may be setup in a variety of configurations to monitor different patch configurations or adhesive joints; however, this technology will be limited to monitoring only the edges. Any disbond that initiated directly underneath the patch may not be detected until patch disbond is severe. 


\section{Chapter 5.0: Conclusions}

This thesis focused on the use of Structural Health Monitoring (SHM) Systems for the detection and quantification of crack and disbond growth. Three SHM systems were tested within this research: Acousto-Ultrasonic (AU), Capacitance Disbond Detection Technique (CDDT) and Surface Mountable Crack Detection System (SMCDS). The AU system used within this research was manufactured by Acellent Technologies Inc. AU is a process in which Lamb Waves (LW) are transmitted through a structure and analysed for the detection and quantification of damage. Changes between current signals and benchmark signals indicate damage formation. The CDDT technology used within this research is a process that has been tested at the National Research Council Canada (NRC) for the detection of disbond. CDDT is a process in which patch adhesion may be quantified by its ability to carry a capacitive charge. Disbond of a patch from a substrate results in a change in capacitance, which may be correlated to the damage size. SMCDS technology is a process in which conductive paint is placed onto a surface which is subjected to cracking. Cracking within the component surface also propagates through the conductive paint, which results into a failed sensor. A failed sensor is detected by its inability to conduct current while under interrogation.

To test these three SHM suites for the detection of crack and disbond growth, three coupon sets were designed. The first set of coupons was made of aluminum and had a center surface crack; these coupons were used for the evaluation of the SHM systems for their effectiveness in crack growth detection. The second set of coupons was made of an aluminum substrate and a carbon fibre patch. Both the patch and the substrate were bonded together; these coupons were for the evaluation of the SHM systems for their effectiveness in crack and disbond growth detection. The third set of coupons was made of a carbon fibre substrate and a boron fibre patch. Both the patch and the substrate were bonded together; these coupons were for the 
evaluation of the SHM systems for their effectiveness in disbond growth detection. All coupons were designed through the aid of Finite Element Analysis. The designed coupons were manufactured and instrumented with SHM sensors at NRC. AU, CDDT and SMCDS were all instrumented on the coupons. Coupons where split in two groups; SMCDS were installed on one and CDDT sensors on the other. However, all coupons were instrumented with AU sensors.

Results of crack growth monitoring using the AU system for coupon Set 1 was analyzed using the DPI and the SEDS utilities. Multiple coupons were tested using this system and analyzed using the two utilities. The DPI utility was able to indicate the initiation as well as propagation of cracks on all tested coupons. In some cases, the DPI utility provided damage identification in the correct location of the crack while in other cases, the identified location was found just below the actual crack. The SEDS utility was also used to interpret crack lengths. For all tested coupons, the results were conservative when compared to the actual crack lengths. These results were based on polynomial approximations using DIs. In some cases, the crack length interpretations contained an interpretation spike within the data. This spike is an outlier which is an overinterpreted crack length. If discarded, the crack length interpretations yielded an exponential crack growth graph similar to that of the actual measured crack length. On some coupons, crack interpretation spikes occurred more than once within the same data set.

Analysing disbond using the $\mathrm{AU}$ system on composite patches adhesively joined to an aluminum substrate was also carried out using both the DPI and SEDS utilities. Multiple sensor configurations were used on multiple coupons. Variances within sensor configurations were utilized to determine if greater scanning accuracy and resolution may be obtained. Through the use of many sensors, damage detection analysis was isolated to specific zones along the coupon. This was done to monitor disbond at the tip, the center and the base of the patch. Based on 
multi-zone (simultaneous zone) analysis, the DPI indicated major disbond along the entire patch; this however was inaccurate when compared to the Non-Destructive Evaluation (NDE) results. The analysis of single zones did result in greater accuracy as compared to the analysis of all zones simultaneously. In cases where only the tip of the patch was examined, NDE results correlated with the DPI results. However, it was found that results for the bottom of the patch were not always accurate. In some cases, the DPI utility indicated disbond where no disbond existed and in other cases it indicated disbond where disbond existed. SEDS utility was also applied to analyze all the coupons and each analysis provided different results. In some cases, disbond size was over-interpreted by a maximum of $375 \%$ and in other cases by a minimum of 45\%. All coupons were identical; however, results were very different for each coupon. In addition to over-prediction of disbond size, some interpretations resulted in an underinterpretation. Under-interpreted disbond areas are dangerous as they provide false information regarding the integrity of the bond.

Monitoring of disbond on composite patches adhesively joined to a composite substrate was done through the use of the AU, CDDT and SMCDS SHM systems. The DPI and the SEDS utilities were used to analyse the disbond area location and size. For these coupons, two sensor layouts were used. For the sensor layout that contained sensors bonded to the substrate and the patch, it was found that the sensors experienced disbond from the substrate as a result of high strain. Sensor configurations which only contained sensors on the patch did not experience sensor disbond. The DPI utility was used to analyze results for both sensor layouts. For the layout which had sensors on the substrate, the DPI utility indicated damage in all areas of the patch. This was inaccurate when compared to the NDE results. These results were attributed to poor signal transmission which was a result of sensor disbond. Coupons with sensors only on the substrate provided better results than those which experienced sensor disbond. In all cases, the DPI utility 
provided damage location and progression identification that was closely correlated to those found using NDE. SEDS utility was also used to analyze the data of all the coupons. For coupons with sensors on the substrate, the disbond area interpretation provided results which were in no way comparable to the actual disbond area. For coupons which had no sensors on the substrate, disbond area interpretations were conservative when compared to the actual disbond areas. In some cases, disbond size was over-interpreted by a maximum of $138 \%$ and in other cases by a minimum of $15 \%$. None of the disbond area interpretations were found to be less than the actual disbond area. CDDT and SMCDS were also implemented to monitor disbond on these patches. In CDDT, capacitance drop was observed as the patch disbond area increased. CDDT results indicated that disbond area may be correlated to change within capacitance and that the trend may be linear. CDDT was found to be useful for the quantification of patch disbond; however, was not useful for the indication of disbond location. The SMCDS was also used and found to indicate where and when the adhesive edges along the patch began to crack. Both the CDDT and the SMCDS techniques could be used together to indicate damage location and size when implemented properly on specific geometries. 


\section{References}

[1] G Wheatley, J Kollgaard, J Register, and M Zaidi, "Comparative Vacuum Monitoring as an Alternate Means of Compliance," in Structural Health Monitoring , 2003, pp. 1358-1365.

[2] V. Giurgiutiu, Structural health monitoring with piezoelectric wafer active sensors. Boston, USA: Academic Press, 2008.

[3] David Roylance, Introduction to Composite Materials. Massachusetts Institute of Technology, Cambridge, USA, March 20, 2000.

[4] Daniel B Miracle and Steven L Donaldson, Introduction to Composites. Air Force Research Laboratory, United States.

[5] Kutz and Mayer, Handbook of Material Selection. New York, USA: John Wiley \& Sons, 2002.

[6] G.B.Murri, "Testing and life predictions of composite materials," U.S. Army Research Laboratory, Vehicle Technology Directorate, Hampton, March 23, 2006.

[7] United States Dept. of Defense, Composite materials handbook. Material usage, design and analysis. Washington, D.C, United States: United States Dept. of Defense, 2002.

[8] A. A. Baker, S. Dutton, and D. Kelly, Composite Materials for Aircraft Structures, secon edition ed.: American Institute of Aeronautics and Astronautics, 2004.

[9] Daniel Feszty, "Rotorcraft," Carleton University, Ottawa, Class Lecture 2009.

[10] Sanjay k Mazumdar, Composite manufacturing: product, and process engineering. Dallas, Texsas: CRC press, 2001.

[11] Ravi B Deo, James H Starnes, and Richard C Holzwarth, "Low-Cost Composite Materials and Structures for Aircraft Applications," in RTO-MP-069(II), Loen, 2001.

[12] Boeing, "Commercial Airplanes - 787 Dreamliner - Background," Boeing, Seattle, Press release 2010.

[13] Richard A Leyes and William Fleming, The History of North American Small Gas Turbine Aircraft Engines, illustrated ed. USA: AIAA, 1999.

[14] Driver Side. (2009, Spetember ) Aston Martin Showes off Some of the One-77 at Geneva. [Online]. http://www.driverside.com/backseatdriver/2009/03/04/aston-martin-shows-offsome-of-the-one-77-at-geneva/comments-page-1/ 
[15] Auto Blog. (2009, Sepetember) Aston Martin One-77 Uncovered: In-depth on what's under the skin. [Online]. http://www.autoblog.com/2009/03/06/aston-martin-one-77-uncoveredin-depth-on-whats-under-the-skin/

[16] David A Nethercot, Composite Construction. New York, USA: Spon Press, 2003.

[17] C Zweben, Composite Materials and Mechanical Design, Mechanical Engineer's Handbook, 2nd ed. New York, USA: John Wiley \& Sons , 1998.

[18] F L Matthews, Joining Fibre-Reinforced Plastics. New York, USA: Elsevier Applied Science, 1987.

[19] R Martin, Ageing of Composites. Cambridge, England: Woodhead Punlishing Limited, 2008.

[20] M D Bannea and LFM da Silvia, "Adhesively Bonded Joints in Composite Matrerial: an Overview," Insituti de Engenharia Mecanica, Porto, Portugal, 2008.

[21] Keith B. Armstrong, L. Graham Bevan, and William F. Cole, Care and Repair of Advanced Composites, 2nd ed.: Society of Automotive Engineers, Inc., 2005.

[22] A.A. Baker, R.J. Callinan, M.J. Davis, R Jones, and J.G. Williams, "Repair of mirage III aircraft using the BFRP crack-patching technique," Theoretical and Applied Fracture Mechanics 2, pp. 1-15, 1984.

[23] A.A. Baker, "Repair of cracked or defective metallic aircraft components with advanced fibre composites," Composite Structures 2, pp. 153-181, 1984.

[24] Satya N Atluri, Structural integrity and Durability. Forsyth, Georgia, USA: Tech Science Press, 1997.

[25] W Baker, I Mackenzie, and R Jones, "Development of life extension strategies for Australian military aircrafts, using structural health monitoring of composite repairs and joints," Composite Structures, vol. 66, no. 1-4, pp. 133-143, October 2004.

[26] Pierre Noel Marty, N Desai, and J Anderson , "NDT OF KISSING BOND IN AERONAUTICAL STRUCTURES," in WCNDT, Linköping, Sweeden, 2004.

[27] Sameer Homoush, Kunigal Shivakumar, Feras Darwish, Mathew Sharpe, and Paul Swindell, "Defective Repairs of Laminated Solid Composites," Journal of Composite Materials , vol. 39, no. 24, pp. 2185-2196, Nov 2005.

[28] Mark Lin, Xinlin Qing, Amrita Kumar, and Shawn J Beard, "Smart Layer and Smart Suitcase for Structural Health Monitoring Applications," in SPIE, Arlington, VA, 22204, 2001, pp. 98 - 
106.

[29] M Martinez et al., "Demonstration of an Instrumented Patch Phase 1," National Research Council Canada, Ottawa, Lab Technical Report LTR-SMPL-2006-0226, 2007.

[30] H. Speckmann and R. Henrich. (2009, June) Structural Health Monitoring (SHM) - Overview on Technologies under development. [Online]. http://www.ndt.net/article/wcndt2004/pdf/aerospace/563 henrich.pdf

[31] H. Speckmann, "Structural Health Monitoring (SHM)," IMRBPB Meeting, EASA, Colonge, Presentation 2007.

[32] W. Staszewski, C. Boller, and G. Tomlinson, Health monitoring of aerospace structures: smart sensor technologies and signal processing. Hoboken, NJ, USA: J. Wiley, 2004.

[33] Su Zhongqing and Lin Ye, Identification of damage using Lamb waves from fundamentals to applications. New York, USA: Springer, 2009.

[34] M Genest, M Martinez, N Mrad, G Renaud, and A Fahr, "Pulsed thermography for nondestructive evaluation and damage growth monitoring of bonded repairs," Composite Structures, vol. 88, no. 1, pp. 112-120, March 2009.

[35] Holger Speckmann and Henrik Roesner, "Structural Health Monitoring: A Contribution to the Intelligent Aircraft Structure," in ECNDT, 2006.

[36] Sjostrom F. (2008, December) The ABCs of Fiber Bragg Gratings. [Online]. http://electronicdesign.com/articles/print.cfm?AD=18.Artic/ID $=20252$

[37] All About Circuits. (2009, August) Strain Gauges. [Online]. http://www.allaboutcircuits.com/vol 1/chpt 9/7.html

[38] National Instruments. (2009, June) Measuring Strains with Strain Gauges. [Online]. http://zone.ni.com/devzone/cda/tut/p/id/3642

[39] M Martinez et al., "Demonstration of an Instrumented Patch - Phase II," National Research Council Canada, Ottawa, Lab Technical Report LTR-SMPL-2008-0135, 2008.

[40] EADS. (2009, August) Structural Health Monitoring. [Online]. http://www.edas.com/1024/en/madebyeads/endurance/shm.html

[41] M Martinez et al., "Surface Mountable Crack Detection System," National Research Council Canada, Ottawa, LM-SMPL-2009-0037, 2009. 
[42] Acellent Technologies Inc. (2010) Software - SmartPatch. [Online]. http://www.acellentsensors.com/Software SmartPatch.asp

[43] M. Scheerer, "SHM of Composite Structures using Acoustic Emission Methods," in European Ground Testing Instrumentation Aerospace Testing Expo, Munich, April 15-16, 2008.

[44] Shin-etsu Fujimoto and Hideki Sekine, "Identification of crack and disbond fronts in repaired aircraft structural panels with bonded FRP composite patches," Composite Structures, vol. 77, no. 4, pp. 533-545, February 2007.

[45] R Jones and R.J Callinan, "A design Study in Crack Patching," Fibre Science and Technology, vol. 14, no. 2, pp. 99-111, February 1981.

[46] D Quinas, B.B. Bouiadjra, B Serier, and M SaidBekkouche, "Comparison of the effectiveness of boron/epoxy and graphite/epoxy patches for repaired cracks emanating from a semicircular notch edge," Composite Structures, vol. 80, no. 4, pp. 514-522, October 2007.

[47] Chorong-Fuh Liu, Horng-Shian Jou, and Ying-Te Lee, "Stress Intensity Factor of Patched Crack," International Journal of Solids and Structures, vol. 24, no. 13, pp. 1557-1562, May 1997.

[48] M Martinez, G Renaud, D Backman, M Genest, and M Delanny, "Demonstration of an instrumented patch," in SPIE, 2007, pp. 65300M-1 TO 65300M-11.

[49] Stephen C Galea et al., "Development of Structural Health Monitoring Systems for Composite Bonded Repairs on Aircraft Structures," in SPIE, 2001.

[50] H Sohn, D Dutta, and Y.K An, "Baseline-Free Damage Detection through Mode Separation of Lamb Waves using Self-Sensing Piezoelectric Transducers," in Structural Health Monitoring, 2009.

[51] Kelah Wakha, Paul Samuel, and Darryll Pines, "A smart composite patch for the repair of aircraft structures," in SPIE, Bellingham, 2005.

[52] M Martinez, L Gang, D Backman, A Oudovikine, and N Bellinger, "Crack detection on composite and metallic aerospace structures," in 4th European Workshop on Structural Health Monitoring, 2008, pp. 560-569.

[53] Gary L. and Kishoni, Doron Workman, Nondestructive Testing Handbook, Ultrasonic Testing, 3rd ed.: ASNT, 2007.

[54] Clemente Ibarra Castanedo, "Quantitative subsurface defect evaluation by pulsed phase thermography: depth retrieval with the phase," Faculte des Sciences et de Genie Universite, 
Laval, Ph.D Thesis 2005.

[55] X Maldague, F Galmiche, and A Ziadi, "Advances in Pulsed Phase Thermography," Electrical and Computing Engineering Dept, University Laval, Quebec City, 2001.

[56] X Maldague and S Marinetti, "Pulse Phase Infrared Thermography," Electrical and Computing Engineering Dept. University Laval, Quebec City, 2001.

[57] Alan Baker, "Structural Health Monitoring of a Bonded Composiite Patch Repair on a Fatigue-Cracked Wing," Deffence Seience and Technology Organisation Victoria, Victoria, Australia, DTSO-RR-0335, 2008.

[58] William Hart Hayt, Jack Ellsworth Kemmerl, and Steven M Durbin, Engineering circuit analysis, 7th ed. New York, USA: McGraw-Hill Higher Education, 2001.

[59] Larry K Baxter, Capacitive Sensors: Design and Applications. New York, USA: IEEE Press, 1997.

[60] J. Stevan, Damage Detection Using Lamb Waves for Structural Health Monitoring. USA: Air Force Institute Of Technology, 2007.

[61] V Giurgiutiu and A Cuc, "Concepts for using Piezoelectric Wafer Active Sensors in Rotorcraft Health Monitoring," in Structural Health Monitoring, 2005, pp. 1173-1182.

[62] A A Baker, Nick Rajic, and Claire Davis, "Towards a practical structural health monitoring technology for patched cracks in aircraft structure," Composites Part A: Applied Science and Manufacturing, vol. 40, no. 9, pp. 1340-1352, Sepetember 2009.

[63] Gareth S Pierce, Brian Culshaw, Graeme Manson, Keith Worden, and Wieslaw Staszewski, "Application of ultrasonic Lamb wave techniques to the evaluation of advanced composite structures," in SPIE, 2000, pp. 93-103.

[64] Shashank Pant, "Damage Detection Using Lamb Waves: Derivation of Lamb Wave Equations for Structural Health Monitoring (SHM) Applications," Carleton University, Ottawa, 2010.

[65] Joseph L Rose, Ultrasonic Waves in Solid Media, 1st ed. Cambridge , England: Cambridge University Press, 2004.

[66] Sourav Banerjee, Shawn Beard, Fady Habib, and Marcias Martinez, "Damage Quantification using Smart Patch System for Hot Spot," in SPIE, 2010.

[67] Preformance Composites Ltd. (2009, July) Mechanical Properties of Carbon Fibre Composite Materials, Fibre / Epoxy resin $\left(120^{\circ} \mathrm{C}\right.$ Cure $)$. [Online]. http://www.performance- 
composites.com/carbonfibre/mechanicalproperties 2.asp

[68] Cytec Engineered Materials. (2010) Cytec, CYCOM 5276-1. [Online]. https://www.cytec.com/engineered-materials/products/Datasheets/CYCOM\%205276-1.pdf

[69] Specialty Materials, Inc. (2010) Specialty Materials, Boron Prepreg Tape Properties.

[Online]. http://www.specmaterials.com/boronprepregproperties.htm

[70] Cytec Engineered Materials. (2011) FM 73 Epoxy Film Adheisive. [Online].

http://www.cytec.com/engineered-materials/products/Datasheets/FM73-081111.pdf 